\title{
A Theory of Subjective Wellbeing
}

Mark Fabian

A thesis submitted for the degree of Doctor of Philosophy of The Australian National University C C Copyright by Mark Fabian 2018

All Rights Reserved 
I certify that this dissertation is my own work, based on my personal study and research and that I have acknowledged all material and sources used in its preparation, whether they be books, articles, reports, lecture notes, and any other kind of document, electronic or personal communication.

I also certify that this dissertation has not previously been submitted for assessment as part of any other programme and that I have not copied in part or whole or otherwise plagiarised the work of other students and/or persons.

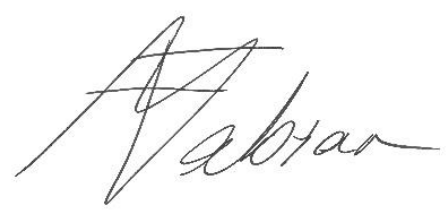

Mark Fabian

Word Count: 91269 


\section{Acknowledgements}

I would like to thank my supervisory panel: Professor Robert Breunig, Professor Carol Graham and Professor Geoffrey Brennan. This thesis is a rather unusual bit of interdisciplinary work and it can't have been easy to shepherd through to completion. More broadly, I want to thank the many colleagues, friends and family who provided support for my self-esteem over the past few years. Doing interdisciplinary research in a relatively young field means that you're something of an intellectual pariah. This can be lonely. Worse, it means that you typically have little other than your own self-belief to keep you going through periods of doubt - you cannot fall back on the fact that you are doing the same work as so and so other scholar and that your scholarly community thinks your work is solid. As such, intermittent enthusiasm, interest and positive feedback from colleagues in general is crucial. I want to particularly thank my chair, Bob Breunig, in this regard. Bob championed my research effectively and worked hard to integrate me into a range of intellectual efforts around the Crawford School. He also agreed to co-author a book on hybrid policies with me, which was a huge boost to my confidence as an early-career academic. I would also like to thank Richard Ryan and Anna Alexandrova, and various scholars of subjective well-being who I met at the Warwick Summer School on Wellbeing, especially Nick Powdthavee who invited me. This list includes especially Ekaterina Oparina, Reto Odermat and Matthew Adler, as well as Jessica Milligan, Paul Frijters and Andrew Oswald. Additionally, I want to extend a thank you to Jan Emmanuelle De Neve, Ed Diener and Arthur Grimes, all of whom I met at the $3^{\text {rd }}$ International Conference on Wellbeing in Wellington, New Zealand, and all of whom had constructive things to say about my research. Peter Drysdale, Shiro Armstrong and Ligang Song provided a nourishing intellectual environment within the development economics and political economy space for me at the Crawford School. They also kept me financially buoyant during my doctoral candidacy with scholarships and tutoring work. Peter deserves a special thank you because he got me into economics in the first place. Finally, I would like to thank my partner Madeline Goldie for putting up with the mood swings that are an inevitable part of any doctoral dissertation and for her emotional support more generally. My PhD was the best 4 years of my life to date and I hope things just keep getting better. 
For my parents, Dorottya Fabian and Janos Fabian 


\begin{abstract}
Subjective well-being scholarship (SW-B) has opened valuable new vistas in wellbeing research over the past four decades or so. However, owing to its operationalist epistemology, it cannot effectively advance into areas it now wants to influence, notably welfare economics and public policy. It must first adopt a more realist epistemology, which begins with a deep theory of wellbeing, including its causal structure. I provide this theory - the wellbeing production function — drawing on ideas in clinical, hedonic, moral, behavioural and developmental psychology, and both analytical and continental philosophy. The individual components of this theory have empirical backing, but the model of wellbeing that it gives rise to produces some inferences that are not verified by subjective well-being data. In particular, the model would suggest that people can experience sustained improvements in life satisfaction over the life course, but this is rarely what we see in data generated by life satisfaction scales questions administered in advanced nations. This discrepancy motivates an investigation in the final chapter as to whether life satisfaction scales might suffer from issues of scale norming driven by ceiling effects. This would explain the discrepancy between the model's inferences and what we see in the data. I provide theoretical arguments and empirical evidence from a novel life satisfaction plotting metric that lends credence to this hypothesis. This leads me to argue that the field should experiment with alternate metrics for measuring life satisfaction and evaluated wellbeing more generally. I discuss some possible options.
\end{abstract}




\section{TABLE OF CONTENTS}

Introduction

Chapter 1-Subjective Well-Being 9

Chapter 2-Theoretical Problems with Subjective Well-Being 24

Chapter 3-The Analytical Philosophy of Wellbeing 48

Chapter 4-A Wellbeing Production Function 69

Chapter 5-The Capabilities Constraint 89

Chapter 6-Hedonia 101

Chapter 7-Eudaimonia 129

Chapter 8-Despair 142

Chapter 9-The Coalescence of Being 178

Chapter 10-Measuring Subjective Wellbeing 206

$\begin{array}{ll}\text { Conclusion } & 243\end{array}$

$\begin{array}{ll}\text { Works Cited } & 249\end{array}$

$\begin{array}{ll}\text { Appendix } & 270\end{array}$ 


\section{Introduction}

Nature has placed mankind under the governance of two sovereign masters: pain and pleasure. It is for them alone to point out what we ought to do, as well as to determine what we shall do. On the one hand the standard of right and wrong, on the other the chain of causes and effects, are fastened to their throne.

- Jeremy Bentham, An Introduction to the Principles of Morals and Legislation

Man does not strive for happiness; only the Englishman does that.

- $\quad$ Friedrich Nietzsche, Twilight of the Idols

There has been a tremendous upwelling of interest in subjective well-being (SW-B) in the past four decades or so, driven in large part by hedonic psychology (Kahneman et al 1999) and more recently by happiness economists (Frey and Stutzer 2002, Bruni and Porta 2005, Weimann et al 2015). This research has been almost entirely atheoretical (Argyle 2001, p. 227) and has relied on an approach that might be described as exploratory data analysis. Researchers began with simple metrics for measuring SW-B, notably life satisfaction and experience sampling tools for studying mood. They delved into the data collected using these metrics with mostly correlational statistical techniques to derive insights into the nature of SW-B. The concept itself was defined operationally by how it was measured: as affect and life satisfaction. Early findings by researchers in the field were built on using richer data sets, and insights were steadily if slowly refined.

This line of inquiry has opened important new vistas. Most significantly, it has convinced a great many people that SW-B can actually be studied empirically, something that economists in particular have been sceptical of since an influential publication by Robbins (1934). It has also brought to light a range of important issues concerning the cognitive evaluation of 
wellbeing, including the possibility of adaptation to new circumstances and the impact of changing reference groups on life satisfaction. Finally, it has freed wellbeing scholarship from the arguably stagnant debates of philosophers and allowed it to be discussed independently from the tricky normative issue of prudential value — what is "good for" someone. In the process, SW-B scholarship has even revitalised philosophical inquiry into wellbeing (Fletcher 2015).

Encouraged by these successes, SW-B research has started to press into the public policy and welfare economics discourses, notably as part of the movement to go "beyond GDP" (Diener and Seligman 2004, Diener et al 2009, Clark et al 2018). In this, SW-B scholarship seems to have overstepped its limits. As calls to more thoroughly integrated perspectives from SW-B research into public policy have increased in volume, critiques of such actions have also increased in quantity and prominence. Old arguments pertaining to the difficulty of measuring SW-B with the precision required for public policy (Adler 2013), the potential for perverse outcomes from "affective governance" (Jupp et al 2016, Davies 2015), and the ethical dubiousness of making SW-B the target of policy have come once again to the fore (Haybron and Tiberius 2015, Fleurbaey and Blanchet 2015, ch. 5).

To date, SW-B scholarship has not responded to these criticisms in detail. This is understandable given the historical context of the field. Subjective well-being had to push past a lot of these old critiques to establish itself. It is now established, and the limits of its present methods and theories are being revealed by its recent attempts to go beyond the parameters it set itself in its infancy. As Alexandrova (2017) describes, SW-B has until now been a "field science" scouting terrain. It discovered a large area of inquiry that could be explored without needing to engage with classic critiques. But now this area is more or less documented and so SW-B scholarship is moving further afield. As it attempts to cross over borders into new territory it is coming up against the classic critiques. To effectively contribute to areas like public policy and welfare economics, SW-B scholarship must address these critiques by revisiting its fundamental methodological limitations, specifically its lack of theoretical depth and the questionable precision of its empirical instruments. In doing so it will transition from being a field science to being a more mature theory-led science.

As I will argue in this thesis, now is an excellent time for this self-critical reflection as the field's efforts in recent decades have granted it the requisite credibility and respect. There is a growing appreciation for the research questions of the field, interest in its findings, and 
patience with its shortcomings. This is indicated by the growing frequency of publications associated with SW-B appearing in top journals across economics, psychology and philosophy. SW-B has attained the latitude required to engage in a period of more speculative, potentially messy research that may undermine the field in the short-term but ultimately strengthen its foundations into the long term.

The present volume is an attempt to kick-off this new wave of scholarship. It provides the holistic theory of wellbeing that SW-B scholarship presently lacks. It also clearly articulates and empirically demonstrates the measurement problems of scholarship to date, and presents novel solutions.

This book develops an integrated theory of subjective wellbeing (SWB - unhyphenated) drawing on ideas in clinical, developmental, moral and behavioural psychology, analytical and continental philosophy, and economics. SWB here refers to how well individuals believe themselves to be. This is distinct from objective well-being, which refers to how well individuals are independently of their own subjective assessment. SWB is also distinct from, or rather broader than, SW-B, which I use to refer only and specifically to the constructs of affect and life satisfaction and to the literatures in hedonic psychology and happiness economics that have studied them. In a sense, the theory developed here merges the field of "subjective well-being" (hyphenated) with the broader literature on "subjective wellbeing" (unhyphenated), such as that which prevails in clinical psychology and philosophy. For the sake of brevity, I will use the acronyms SW-B and SWB for subjective well-being and subjective wellbeing, respectively. I will use the term "wellbeing" to describe all research concerned with the topic and all conceptualisations of it, including SW-B and those lines of inquiry concerned with objective wellbeing. I will use the term "welfare" to refer specifically to matters concerning the standard of living. Welfare thus takes in just about all of the literature in economics concerned with wellbeing other than happiness economics.

The broad theory of SWB presented in this book has two parts. The first is a description of wellbeing as an outcome. This aspect of the theory is called the wellbeing production function. Wellbeing is here modelled as a dependent variable that is a function of three "dimensions of wellbeing" that serve as independent variables. These are hedonia, eudaimonia and despair. These three dimensions roughly correspond to whether life is pleasant, fulfilling and valuable, respectively. Each dimension is made up of further subvariables. Hedonia is a function of positive and negative affect and hedonic life satisfaction. 
Eudaimonia is a function of the three basic psychological needs of autonomy, relatedness and competence. And despair is a function of the three themes of existentialism: meaning and purpose (nausea), identity (anguish), and virtue (seriousness).

The second part of the theory is a model of the process by which wellbeing is attained. This aspect of the theory is called the coalescence of being. It is a model of self-actualisation in the eudaimonic tradition, blending insights from psychology and philosophy. Coalescence involves the individual harmonising their actual self with their ideal and ought selves through goal setting and achievement. This process is guided by affective signals that accompany goal pursuit and social self-verification. Introspection upon these signals helps the individual to comport towards an identity that is self-concordant and wellbeing promoting.

This two-part theory is a "mega-hybrid" theory of wellbeing. In recent accounts from analytical philosophy, hybrids are characterised as theories of wellbeing that blend two or more of the traditional schools of thought regarding the nature of wellbeing, namely hedonism, objectivism and subjectivism (Woodard 2015). The production function of wellbeing is both objectivist and subjectivist in that it emphasises objective criteria that define wellbeing but also recognises that how these criteria are met will differ from individual to individual. In short, the production function of wellbeing posits objective ends with subjective means. The coalescence of being then places a strong emphasis on process, arguing that there is a prudent way to pursue wellbeing, albeit one that allows for a great deal of individual heterogeneity. This integrates eudaimonic perspectives on wellbeing into the theory of subjective wellbeing. Eudaimonists emphasise how one must live in order to attain wellbeing (often described as flourishing in this literature), rather than dwelling on what kind of state wellbeing is (Besser-Jones 2015). Coalescence also sees a strong role for virtue in the form of the ought self and reason in the form of introspection, thereby bringing into relief the Aristotelian emphasis on rationality and virtue in fostering wellbeing. Finally, coalescence delineates a critical role for affective signals in steering self-actualisation. This means that hedonism is central to a holistic understanding of wellbeing. I thus argue in this thesis that hedonism, subjectivism, objectivism and eudaimonia are all interdependent in wellbeing. Understanding wellbeing thus requires integrating the insights of each of these schools into an internally consistent model. This in turn requires a strongly cross-disciplinary approach.

The theory of wellbeing presented herein is unique in that it is integrative, interdisciplinary and describes wellbeing in terms of its causal structure. It complements existing statistical 
studies of the overarching structure of wellbeing (Ryff 1989b, Van Dierendonck 2004, Springer et al. 2006, Springer and Hauser 2006, Diener et al 2010, Hupert and So 2013) as an outcome variable with a sophisticated theoretical account of how different aspects of wellbeing interact as part of the process by which wellbeing is achieved. In so doing, it clarifies (i) why certain variables overlap in correlational analysis while others do not, (ii) the nature of apparent two-way causal relationships between wellbeing variables, and (iii) how the many seemingly divergent conceptions of wellbeing in philosophy, psychology and economics can be harmonised in a way that brings clarity rather than merely added complexity.

This thesis is structured as follows. It begins with an account of SW-B including its history and positive contributions to scholarship. The history of the field is important because it explains why SW-B proceeded with its peculiar methodology and why it was helpful and appropriate that it did so. The second chapter reviews theoretical (as opposed to empirical) weaknesses in the paradigm of SW-B. Foremost among these is that SW-B's operationalist epistemology gives rise to circularity. Construct validation exercises are pushed into service to resolve this issue. However, construct validation requires theorising at step one and this theorising is lacking in SW-B, at least for the new lines of inquiry it now wishes to explore. SW-B's operationalist epistemology is particularly problematic in the context of studying SWB because it gives rise to what Alexandrova (2017) calls "evidential subjectivism", which is where both the definition of the construct of interest and the way it is measured are subjectively defined. It is logically impossible to ensure that you are analysing a consistent construct when utilising an evidential subjectivism paradigm. These epistemic issues point to the need for deeper theorising in order to bridge SW-B and SWB. This deeper theory is elaborated over the next seven chapters.

The theoretical exposition begins with an exploration of analytical philosophy's perspectives on wellbeing. These define wellbeing as the prudential good, meaning what is "good-for" an individual. There are three traditional classes of wellbeing theory in analytical philosophy: hedonism, subjectivism and objectivism. Chapter 3 argues that analytical philosophy's tendency towards differentiation and classification has predisposed it to overlook important complementarities between these different classes. These complementarities are manifesting in tightening equivalence between the three classes in recent cycles of debate between their advocates. Focusing on the overlaps between the three classes reveals that they are more interdependent than their advocates and analytical philosophers in general might presume. 
This motivates the production function model of wellbeing, which tries to integrate rather than differentiate various perspectives on and theories about happiness. Chapter 3 also argues that analytical philosophy has tended to emphasise wellbeing as an outcome and thereby miss the crucial prudential issues associated with how wellbeing is pursued. There is a right way of achieving wellbeing regardless of how wellbeing is defined. Understanding this process requires integrating perspectives from the eudaimonic branch of wellbeing theories. To date, these have mostly been discussed under the rubric of objectivist theories of wellbeing as outcome, often missing their insights into wellbeing as process.

Chapter 4 presents the production function model of wellbeing in full, including a formal mathematical specification. It provides a preliminary description of the components of the production function. Chapters 5 through 8 then explain these components in more detail and justify their inclusion in the model. The production function model of wellbeing extends the consumer's problem in economics from one where an individual maximises their utility from consumption subject to a budget constraint to a broader problem where the individual tries to maximise their wellbeing subject to a capabilities constraint and a lack of wisdom and information regarding what will enhance their wellbeing.

The individual's capability constraint consists of income, health, education, political enfranchisement and environmental quality. These variables are justified in chapter 5 with reference to the development studies literature, particularly the work of Sen (1999a, 1999b). The individual's wellbeing function is given by the wellbeing production function and consists of hedonia, eudaimonia and despair.

The nature and content of hedonia is elaborated in chapter 6 drawing especially on the work of hedonic psychologists and happiness economists. This chapter not only describes hedonia but also, continuing the emphasis in chapter 3 on the praxis of wellbeing, explains how to get it. This explanation covers a range of techniques for mood management developed in recent decades including gratitude, savouring, positive activity interventions, mindfulness and prosociality.

Chapter 7 continues work started in chapter 6 differentiating hedonia and eudaimonia. It also goes into more detail on the nature of the three eudaimonic variables: autonomy, competence and relatedness. These are the basic psychological needs postulated by self-determination theory (SDT), the most prominent school of eudaimonic psychology. SDT has a tremendous amount of wisdom to contribute to our understanding of the process by which wellbeing is 
achieved and individuals come to flourish. These are presented at some length in this chapter. They include the nature of human motivation, how values can be internalised over time, and what goals nurture well-being and why.

Chapter 8 analyses the despair dimension, which concerns the three problems of existential philosophy: nausea, anguish and seriousness. Succinctly and crudely, nausea refers to the need for life to feel meaningful and purposeful. Anguish refers to the need for identity. And seriousness refers to our need to feel like we are a good person and be regarded as such by our peers. SW-B scholarship has recently moved rapidly to integrate meaning and purpose into its understanding of SWB, but it has done this in a typically atheoretical way. This is inappropriate because meaning and the other variables of despair concern fundamentally normative and meta-ethical issues that cannot be understood without substantial theorising. Existential philosophy from roughly 1850 to $1950 \mathrm{did}$ a tremendous job developing this theory, and several more recent literatures in psychology, such as logotherapy, have extended the existentialists' insights. Chapter 8 integrates these works into the broader SWB literature, bringing into sharper focus the importance of despair, how it relates to the other dimensions of wellbeing and what can be done to ameliorate it.

Chapter 9 presents a relatively complete account of the coalescence of being, building on ideas from SDT presented in chapter 7 and ideas from existentialism presented in chapter 8 . The coalescence of being models how agents come to gain the wisdom and information, in particular the self-knowledge, required to leverage their capabilities to attain wellbeing. The coalescence of being is a model of self-actualisation - the process by which we discover, create, affirm and become our identity. Coalescence involves goal setting with the intention of becoming one's ideal self. As the individual goes about achieving these goals they will be met with successes and failures and these will be accompanied by affective signals like depression, anxiety, exhilaration and joy. These signals help the individual to navigate away from goals that are not self-concordant and towards goals and an ideal self that is inspiring and possible for the individual. Steady progress towards being this ideal self in reality brings with it a preponderance of positive affect and gradually reduces instances of negative affect in life, nourishes the basic needs for autonomy, competence and relatedness, overcomes nausea through intrinsic motivation, grants seriousness through the need for integrity and fealty towards the desired-self, and dissolve anguish by building an internally consistent, palpable and rationally accessible sense of self. Coalescence thereby carries us to wellbeing. 
Chapter 10 uses the model of wellbeing developed in chapters 3 to 9 to reflect on how wellbeing is measured. It begins with an analysis of whether life satisfaction scales suffer from a problem of scale-norming. This is where respondents recalibrate their scales between responses over time such that the numbers on their scales correspond to different absolute levels of satisfaction from one survey to the next. The existence of scale-norming would undermine the inter-personal and inter-temporal comparability of responses, thereby making them unsuitable for welfare analysis and most policy applications. The chapter presents existing empirical evidence and new evidence from a novel SWB metric - the plotting metric - that scale-norming is a real phenomenon. These results align with inferences from the coalescence of being. Coalescence suggests that people frequently recalibrate their scales when they experience success or failure to achieve goals. The final part of the chapter elaborates a second new metric for measuring the dynamics of wellbeing-worms. A methodology is then described by which worms could be used to estimate the coefficients of the wellbeing production function and to begin studying how individuals learn what values are most suitable for them as coalescence proceeds.

The conclusion briefly returns to the issue of wellbeing and public policy. It argues that SWB is not ready for applications in welfare economics, notably cost-benefit analysis. However, there are some applications of SWB in the areas of education, workplace organisation, prison policy, and support for community and deliberative mechanisms that could be promoted today without transgressing the ethical obligations of government or being guilty of rushing to apply unripe science. 


\section{Chapter 1}

\section{Subjective Well-Being}

Policy decisions at the organizational, corporate, and governmental levels should be more heavily influenced by issues related to well-being - people's evaluations and feelings about their lives.

- Ed Diener and Martin Seligman, Beyond Money: Towards an Economy of Well-Being

\section{Introduction}

The purpose of this chapter is to review the paradigmatic approach to understanding wellbeing adopted by the subjective well-being literature (SW-B), which dominates contemporary policy thinking on wellbeing among hedonic psychologists and happiness economists. The chapter begins with a brief history of the study of wellbeing in order to explain why SW-B adopted its peculiar paradigm in the first place. SW-B scholarship emerged at the end of the 1960s against a backdrop of logical positivism and scepticism towards anything that wasn't objectively measurable. Economics had just about completely done away with the mind in its theories and while the cognitive revolution was well underway in psychology, neither this new paradigm nor the old behaviourist one could effectively accommodate research into the kinds of questions subjective well-being scholars were interested in. Meanwhile, philosophical and psychoanalytic theorising about wellbeing was hundreds of years old and mired in a swamp of theoretical conjectures so dense as to be difficult to apply in the context of scientific practice. In order to survive in this hostile environment, SW-B scholarship had to adopt a radically empirical methodology with a borderline disdain for theorising. Early work in happiness economics developed alongside this literature and was then increasingly folded into it until today happiness economics is basically a sub-discipline of SW-B scholarship. 
The second part of the chapter explores the definition of wellbeing used in SW-B scholarship. This definition emphasises experience and evaluated wellbeing, typically operationalised if not explicitly defined in terms of affect and life satisfaction respectively. The merits of this definition are discussed in the third part of the chapter, which highlights three main strengths: generality, the clear delineation between experienced and evaluated wellbeing, and liberalism. The focus on very general outcomes in the definition of subjective well-being leaves it free of theoretical priors regarding the ingredients and causal structure of wellbeing. This allowed subjective wellbeing scholarship to break away from the weighty history of wellbeing theorising and focus on empirically validating claims, something philosophy in particular was disinclined to do. The emphasis on experienced wellbeing contrasted with philosophical views, which tended to either dismiss experienced wellbeing as unimportant or argue that it was a function of evaluated wellbeing, neither of which appear to be true. The subjectivity of the definition allows people to decide whether they are well and what exactly this means. This liberal quality inoculates subjective well-being from some forms of political abuse.

This chapter analyses SW-B and presents its merits. The next chapter then discusses its shortcomings, especially in the context of economics and public policy. This discussion motivates a more thorough theoretical engagement with the concept of wellbeing that goes beyond the subjective well-being literature. This called-for theoretical engagement then forms the middle chapters of the book.

\section{A brief history of wellbeing research}

Scholarship of SWB in general but especially among economists is presently dominated by the frameworks developed in the SW-B literature. It is important to distinguish this literature from the broader literature on wellbeing and even on SWB. Ultimately, my contention is that economics, and public policy even more so, should be interested in SWB more broadly than the narrower domain of the SW-B paradigm. The SW-B literature is and will continue to be immensely valuable to furthering our understanding of wellbeing in general, but it has shortcomings when it comes to applications of wellbeing in economics and policy. In part, what this dissertation amounts to is an attempt to fold the subjective well-being literature into the broader literature on SWB. One thing about this integration that will be confusing is that the literature on wellbeing in general contains a rich vein of writings about so-called 
"subjectivist" conceptions of wellbeing. The theory of wellbeing that I present in this dissertation is also a subjectivist theory. The paradigm of the SW-B literature is certainly subjectivist in flavour, but it developed independently of the subjectivist tradition and remains to this day meaningfully distinct from it, as we will see. Succinctly, the SW-B paradigm defines wellbeing (and happiness) as a preponderance of negative over positive affect and high life satisfaction. This is narrower than just restricting one's field of inquiry to subjective assessments of one's own wellbeing, which is what SWB is. I will argue throughout this thesis that SWB is broader than the definition used in SW-B research. Parfit (1984) influentially distinguished between 3 different categories of wellbeing theory: objectivist, hedonistic and subjectivist. Objectivist theories state some collection of criteria that must be met in order for someone to qualify as well. These might be as straightforward as good health, or consider more complex factors like whether the individual is living virtuously. Hedonistic theories argue that wellbeing can be reduced to some balance of pleasure over pain or pleasant over unpleasant mental states. Subjectivist theories put an emphasis on whether an individual considers themselves to be well. Frequent expository tools in the subjectivist tradition are the suffering artist and the stoic or ascetic. The suffering artist cares little for their poor physical health and even for their happiness (mood), and is concerned only with the creation of their works. Similarly, the stoic and ascetic explicitly do not care about the pleasantness of their existence, only that they are free from desire or living in accordance with their religious ideals. On hedonistic conceptions of wellbeing, neither of these individuals is well, but this seems perverse. A similar outcome emerges from the application of most objectivist theories to the stoic and artist.

Objectivist theories dominated classical philosophical perspectives on happiness and wellbeing, such as in the writings of Aristotle and Christians like St. Augustine. They are also very prominent in contemporary thinking around health outcomes and quality of life, and in welfare economics. The capabilities approach, which is ascendant in welfare economics, draws heavily on Aristotelian thinking and, in some of its stronger forms, lays claim to universal values that underpin inter-subjective comparisons of welfare (Nussbaum 2000). In even its weakest forms, it argues that certain objectively observable factors, such as health and health human rights, constitute wellbeing or at least welfare.

Hedonism was largely dismissed in the earliest days of philosophy, notably by Aristotle, who said pleasure was the concern of swine not men. It enjoyed a substantial revival under the 
influence of Bentham and the other early utilitarians. It was, perhaps consequentially, integral to postulates in early economics, which held that people make consumption decisions based on expected outcomes in terms of pleasure or at least positive sensations (Bruni and Sugden 2007). However, hedonism was largely ejected from economics following, first, Mill's revival of Aristotle's swine argument and subsequent delimitation of higher and lower pleasures, and then especially after Robbins' (1934) influential argument that "utility" was unmeasurable and so economics should focus on observed behaviour and simply infer utility from that. Thereafter, utility in economics went from being thought of as something like pleasure to being seen instead as anything to which one could affix a positive $(+)$ sign. Curiously, this would seem to return to a conception of utility that is found in Bentham (1780). He is often considered to have cared only about pleasure and pain as a result of his famous claim that "nature has placed mankind under the governance of two sovereign masters, pain and pleasure". But less than a paragraph after this infamous statement he makes comments that suggest that he uses pain and pleasure as shorthand for the more general categories of value and disvalue:

The principle of utility recognises this subjection, and assumes it for the foundation of that system, the object of which is to rear the fabric of felicity by the hands of reason and law...By utility is meant that property in any object whereby it tends to produce benefit, advantage, pleasure, good or happiness, (all this in the present case comes to the same thing) or (what comes again to the same thing), to prevent the happening of mischief, pain, evil or unhappiness to the party whose interest is considered: if that party be the community in general, then the happiness of the community: if a particular individual, then the happiness of that individual. (ibid. p. 2, emphasis added)

Bentham here seems to be suggesting that utility means anything that is "good" and disutility anything that is "bad". His desire is to make practical statements about policy, not to get bogged down in a debate about what the good is. This approach of treating utility as anything positive was then prosecuted to its logical extreme by Samuelson $(1938,1948)$ in his revealed preferences approach, where utility comes to be only a representation of a preference ordering and utility as some state of mind is completely removed from economics. Economics consequently became associated with a subjectivist theory of wellbeing where individual's satisfying their personal preferences was taken to be wellbeing. 
The history of happiness economics is substantially a history of bringing utility as a state of mind back into economics. Easterlin (1974) first used measures of life satisfaction in the world values survey as a proxy for utility to examine the effect of income. And then Van Praag and his Leiden School (Van Praag and Frijters 1999) used similar assumptions about synonymy to estimate utility functions.

While early work in happiness economics like that of Easterlin and Van Praag developed independently of the SW-B literature, in recent years the two have basically moved in parallel. Indeed, it is fair to say that SW-B scholarship is comprised of hedonic psychologists and happiness economists. Both place a strong emphasis on empirics and exploratory data analysis and are generally disinclination to engage with the theory of wellbeing. With few exceptions, happiness economists use the SW-B definition of wellbeing. Both happiness economists and hedonic psychologists commonly use happiness, life satisfaction and wellbeing interchangeably. And prominent happiness economists frequently appear in academic volumes edited by subjective wellbeing scholars. For example, Van Praag has a chapter in Kahneman et al's 1999 volume (Van Praag and Frijters 1999). Easterlin (2014) has a chapter in Sheldon and Lucas' 2014 volume Stability of Happiness, which in many respects is an updated handbook of hedonic psychology. Powdthavee and Stutzer (2014), two other prominent happiness economists, also have a chapter in that volume. Finally, the list of academic advisors for the OECD's (2017) Guidelines for Measuring Subjective Wellbeing features a substantial number of economists, including doyens of happiness economics like Layard, Helliwell and Krueger.

The arguments of Robbins and the approach of Samuelson mirror in some ways the behavioural turn in Anglophone psychology. Metaphysical questions pertaining to the nature of mind were thought to be impeding progress on understanding human psychology. To remedy this, an emphasis was placed on observable behaviour and its explanations. An individual's subjective reports were irrelevant. This attitude persisted to some extent through the cognitive revolution. As Kahneman et al (1999) note, even the relatively simple or narrow model of wellbeing advocated by hedonism could not be accommodated within the strictures of these paradigms of psychological science.

While hedonistic and more recently objectivist theories of wellbeing predominated in the history of Anglophone thinking about wellbeing until the rise of economic subjectivism, continental philosophy was fonder of subjectivist theories of wellbeing from early on. 
Existentialist philosophy and early psychoanalysis argued for the importance of individuals affirming their personal values and self-actualising in order to achieve wellbeing. Subjectivist conceptions of wellbeing have only become popular in Anglophone philosophy in recent years, perhaps in part as a result of the progress of the subjective well-being literature. Prominent examples from recent decades include Sumner's (1999) highly influential subjectivist "life satisfaction" theory, and the recent work of Vitrano (2014).

It was against this backdrop of deeply qualitative theorising about wellbeing in the history of philosophical thought and widespread antipathy towards subjective or otherwise unobservable phenomena in economics and psychology that the study of subjective wellbeing had to emerge. As Diener et al (2009, p. 15) outline:

In Psychology, several researchers were developing and testing affective theories of well-being in the first decades of the $20^{\text {th }}$ century (Beebe-Center, 1932). Behaviourism ended this program of research, and the scientific study of feelings in psychology reemerged only gradually in the 1960s (Bradburn 1969, Schacter and Singer 1962, Nowlis 1965). It was only after the affective revolution in the 1980s that psychologists rediscovered feelings as an important research topic (Bower 1981, Frijda 1986, Schwarz and Clore 1983).

SW-B seems to have gone about establishing itself in this context by strongly prioritising empirics. It began its research program by developing instruments for measuring the cluster of phenomena it was interested in, notably emotions, affect and satisfaction. A theory of subjective wellbeing was only developed later and only on the basis of what could be observed in data that had been collected. Kahneman, Diener and Schwartz's 1999 volume, Wellbeing: The foundations of hedonic psychology, was the first substantial statement of theory in SW-B scholarship. Even that volume is arguably light on conjecture. SW-B scholarship tends to progress from measurement to theory rather than in the more conventional direction of theory to empirical test. By utilising this data-driven approach, SWB scholarship was able to fend off critics who disliked the study of subjective phenomena due to their inaccessibility, and progress without getting bogged down in the reams of theory that dominated philosophical and psychoanalytic perspectives on wellbeing. 


\section{Definitions of subjective well-being}

It is understandable given this historical background that SW-B scholarship employs a very general definition of what SW-B actually is. Below, I survey several prominent examples of this definition before commenting on its strengths.

One of the most recent and comprehensive engagements with the SW-B literature is the Organisation for Economic Cooperation and Development's 2017 report Guidelines for Measuring Subjective Well-being. It defines wellbeing as:

Good mental states, including all of the various evaluations, positive and negative, that people make of their lives and the affective reactions of people to their experiences. (OECD 2017, p. 10)

This is one of the most general definitions of SW-B in its emphasis on "good mental states" broadly defined and "all the various evaluations" that people make of their lives. The definition apparently draws on Diener et al (2006), Beyond the Hedonic Treadmill: Revisiting the Adaptation Theory of Wellbeing. That article uses a more circumspect definition of wellbeing (note that happiness is here used synonymously with wellbeing and SW-B):

The idea of happiness set points implies that well-being is a single entity with a single baseline. However, work by Lucas, Diener and Suh (1996) indicates that the global category of happiness is composed of separable well-being variables... Thus, the idea of a unitary set point is not tenable, because positive and negative emotions might both decline in tandem or life satisfaction might move upward while positive emotions decrease. (p. 307, emphasis added)

This definition of subjective well-being as a combination of positive effect, negative effect and life satisfaction is standard in the field. It originates with Ed Diener, and its pre-eminence is perhaps due in large part to his leadership of SW-B scholarship from the beginning. A similar definition is provided in Diener et al's (2009, p. 20) volume Wellbeing and Public Policy:

In short, we have defined well-being as a life that matches an individual's own ideals. We think of income, affect, and well-being judgements as alternative indicators of well-being. These indicators reflect well-being for different reasons and they have their own biases and measurement problems. By integrating the 
information across different indicators, it is possible to obtain a better impression of individuals' and societies' well-being.

This definition has a few noteworthy features. First, it is a definition of well-being, not just SW-B. The only difference seems to be the inclusion of income alongside affect and wellbeing judgements in the definition of well-being. Income is an objective indicator of wellbeing, so this cannot be a definition of SW-B. The other variables are the usual combination of affect and some measure of life satisfaction. It is worth noting given the earlier discussion of convergence between hedonic psychology and happiness economics in SW-B that one of the co-authors of Wellbeing and Public Policy is John Helliwell, a prominent happiness economist and author of the World Happiness Report. Other economists writing in the SW-B space give similar definitions of SW-B. The definition employed by the Stiglitz-Sen-Fitoussi commission (2009, p. 16) is perhaps the most succinct:

Subjective well-being encompasses different aspects (cognitive evaluations of one's life, happiness, satisfaction, positive emotions such as joy and pride, and negative emotions such as pain and worry): each of them should be measured separately to derive a more comprehensive appreciation of people's lives.

Again we see an emphasis on affect and cognitive evaluations. The most commonly operationalised such evaluation is life satisfaction, as can be seen from the slightly more complex definition of SW-B below from Clark et al (2017, p. 3):

In our view, we should evaluate people's happiness as they themselves evaluate it. People are often asked, "Overall, how satisfied are you with your life these days?" They answer on a scale of $0-10$, where 0 means "not at all satisfied" and 10 means "extremely satisfied."... When people answer this question, they are evaluating their own overall well-being. That is why we like this question. But well-being is often measured in other ways. One approach is to try to catch people's mood - their current hedonic feelings of enjoyment or discomfort...A third approach is to ask people how worthwhile they consider the things they do in their life - the measure of so-called eudaimonia. These measures are interesting, but we prefer life satisfaction as our measure of well-being for a number of reasons.

This definition demonstrates economists' tendency to think of well-being, life satisfaction and happiness as basically the same thing. It is also notable in that it doesn't so much define 
wellbeing as argue for a particular measure of it, namely life satisfaction as opposed to eudaimonic or affect-based measures. This is a central feature of the subjective well-being literature in general: the construct of interest is basically defined in terms of the measurement instruments used. A final noteworthy feature of Clark et al's definition is that eudaimonic aspects of wellbeing are considered alongside the more traditional affect and satisfaction aspects. This reflects the growing awareness among SW-B researchers of the importance of eudaimonic aspects of wellbeing. Note, for example, that Diener et al's definition in 2009 makes no mention of eudaimonia.

By the time the OECD guidelines were published in 2017, there was a growing awareness of eudaimonia among SW-B scholars. However, the guidelines avoid a full-blown engagement with eudaimonia owing to the relatively embryonic understanding of eudaimonia among SWB scholars. The guidelines document has this to say about eudaimonia in terms of defining wellbeing:

However, the guidelines also provide advice on measuring people's experiences and evaluations of particular domains of life, such as satisfaction with their financial status or satisfaction with their health status, as well as measures of 'meaningfulness' or 'purpose' in life (often described as 'eudaimonic' aspects of subjective well-being). This definition of subjective well-being hence encompasses three elements:

- Life evaluation - a reflective assessment on a person's life or some specific aspect of it

- Affect - a person's feelings or emotional states, typically measured with reference to a particular point in time

- Eudaimonia - a sense of meaning or purpose in life, or good psychological functioning.

It is worth summarising the themes that these definitions of SW-B share. First, SW-B is commonly and classically defined as a combination of experienced and evaluated wellbeing. These are then defined as affect and life satisfaction respectively. Eudaimonic wellbeing, defined as purpose or very broadly as good psychological functioning, is gaining traction as an additional element. Second, SW-B reflects an individual's own self-assessment of their well-being. In the 2009 definition of Diener et al., it also includes a component that is the 
individual's evaluation of the extent to which their life aligns with their ideals for that life. Clark et al (2018) make a similar point shortly after introducing their definition. They say that they prefer to focus on life satisfaction because "it allows individuals to assess their lives on the basis of whatever they consider important to themselves" (p. 4). This is in contrast to objective indicators like GDP and the human development index, which could be improving even as someone complains about the deteriorating quality of their life. Clark et al (ibid.) argue, fairly, that there is an important democratic element to giving primacy to people's own assessments of their quality of life.

\section{The strengths of the subjective well-being approach}

There is a lot to commend in the empirical flavour of SW-B scholarship and its associated usage of a very general definition of the outcome variable of interest. First, the generality of the definitions of wellbeing in SW-B scholarship mitigates against the presupposition of a specific theory of wellbeing. In particular, they say almost nothing about the causes of wellbeing. This generality allowed the first generation of SW-B research to place a strong and overdue emphasis on empirical approaches to improving our understanding of wellbeing. Second, the definition neatly delineates between experienced and evaluated wellbeing, which is important for understanding a range of findings in behavioural and hedonic psychology. Historical definitions, especially in philosophy, rarely made this distinction, or else were somewhat dismissive of experienced wellbeing as compared to evaluated wellbeing. ${ }^{1}$ This cynicism towards affective issues is implicit in Aristotle's disdain for hedonia and in continental (Nietzsche 1889/1990) and analytical philosophy's (Haybron 2001) scepticism about the importance of hedonism in accounts of the good life. Finally, the definition emphasises the subjectivity of wellbeing. This allows individuals themselves rather than some class of enlightened ones to decide whether they are well and what well means.

The definition of subjective well-being as affect and satisfaction posits only indicators of wellbeing and is thus a very open definition of the concept. As Sheldon (2013, p. 132) explains, it does not strongly imply particular ingredients for wellbeing or propose how these ingredients translate into wellbeing:

\footnotetext{
${ }^{1}$ The tendency in philosophy is instead to distinguish between mental-state and state-of-the-world conceptions of wellbeing
} 
Tiberius also calls for a general definition of well-being that does not presuppose a particular theory, just as I do (which is one reason I use the subjective wellbeing measure). However, in my view she may make a mistake in saying that eudaimonic measures describe ingredients "that go beyond positive affect and life-satisfaction". If she means that eudaimonic theories (e.g. the self-concordance model) specify ingredients for happiness that go beyond hedonic theories, I agree. However, if she means that positive affect and life satisfaction (and low negative affect) are insufficient as indicators of well-being, then I disagree, for reasons already discussed. These three variables, combined, provide an admirable criterion for studying what produces happiness, in part because they are content free and do not presuppose a particular and in part because they really do discriminate between hedonic and eudaimonic activities. In a similar vein, Tiberius states that "psychologists who study subjective well-being...take the ingredients of well-being to be subjective, psychological states such as life satisfaction or positive affect". Again, I disagree; I am a psychologist who studies subjective well-being, but I take states of satisfaction and positive affect as the outcome to be predicted by the proper ingredients, not the proper ingredients themselves.

An important benefit of this approach is that it discourages empirical work from becoming biased (in the partisan rather than statistical sense of the word) by theoretical priors. This is arguably a double-edged sword, as we shall see in the next chapter, but makes sense in the historical context of SW-B scholarship. The definition puts to one side the long history of theorising about well-being and effectively hits reset, restarting this field of inquiry from a much more empirical-oriented basis. In the absence of this focus on outcomes and the attendant reset of scholarship, inquiry into wellbeing had a tendency to get rapidly derailed by arguments emerging out of theoretical biases. For example, non-hedonists were liable to be dismissive of empirical inquiry into pleasure-based models of well-being on the grounds that, among other things, affective states are merely the phenoumenal surface of deeper constructs like virtue and self-actualisation (Haybron 2001). It is these deeper constructs that determine hedonic states and it is thus these deeper constructs that constitute wellbeing and are the proper objects of study. A good example of such objections to simplistic theories of well-being is Annas' (2004) Aristotelian critique of subjective well-being scholarship. 
Differentiating between experienced and evaluated wellbeing is important to make sense of a range of empirical observations in behavioural and hedonic psychology. A prominent example is the peak-end rule, where people tend to judge how pleasant or unpleasant an experience was based on its most intense point and/or how the experience felt at its conclusion (Kahneman 1999). As a result, people can remember experiences that they judged at the time to be very painful on average for a relatively long time as being less unpleasant than shorter and less painful on average experiences so long as the worse-at-the-time experience had a weaker peak or a less painful conclusion. This is important for understanding SWB over time, such as in the context of goal pursuit. Many goals are mostly arduous but come with a substantial, albeit brief, final payoff. Completing a $\mathrm{PhD}$ is, for example, arduous but comes with a nice high when you finally tender your thesis and go through your graduation ceremony. It may seem that completing a $\mathrm{PhD}$ is not worth it from an experienced well-being point of view. It is instead typically justified from an evaluative point of view. Completing a $\mathrm{PhD}$ thesis, unlike say, completing a professional license course that you don't care about, has diffuse positive benefits after its peak experience. Besides the graduation ceremony - the peak - one has generally better self-esteem as a result of completion, and a clear symbol one's identity as a smart person and effective scholar. These certainly improve evaluated wellbeing, but may also provide a flow of positive affect when reflected upon or else bolster one's emotional resilience in times of crisis.

Historically, wellbeing theory was arguably dismissive of experienced wellbeing, or at least argued that experienced wellbeing was substantially a function of evaluated wellbeing. For example, Nozick's (1974) famous “experience machine” thought experiment was frequently used to debunk the idea that experienced wellbeing was of primary importance. The experience machine is a fictional device that you can plug into and be transported to some simulated reality, like in the movie The Matrix, wherein you have only positive experiences. In some other iterations of the thought experiment it is even implied that you can live out all your dreams, including the experience of achieving your goals. Would you plug in?

Philosophers generally assume that people would not plug in because they would rather lead a life that is in contact with reality. This implies that hedonic experiences aren't preeminent in people's subjective wellbeing. There is a common argument in philosophical discussions of wellbeing that suggest that an illusory life or one in which the individual is deceived is somehow worse than a life lived with perfect knowledge (see Kagan 1984 and Adams 1999, p. 84, for examples). However, in empirical studies of the experience machine thought 
experiment, people turn out to be more comfortable with the idea of living in The Matrix than philosophers typically suppose (De Brigard 2010, Weijers 2014). Evidence from experience machine experiments suggest that people are certainly fond of reality but that they are also tempted by the machine, and will overwhelmingly choose the machine if reality is unpleasant, such as being consigned to prison (Hindriks and Douven 2018).

A different critique of experienced wellbeing is that it is merely a function of evaluated wellbeing, or at least of things that determine life evaluations. This sort of argument is implied in, for example, self-discrepancy theory, which will be discussed in greater detail in chapter 9. Self-discrepancy theory argues that people try to harmonise who they actually are with who they would ideally like to be. If they achieve this, they experience positive affect in the form of self-esteem and a sense of achievement, while if they fail, they experience depression. The affective states are here the product of an evaluation, specifically whether one is one's ideal self.

The problem with finding the root cause of experienced wellbeing in evaluated wellbeing is that there are undeniably ways for experienced wellbeing to change, at least temporarily, independently of changes in evaluated wellbeing. Many of the techniques of mood management discussed in chapter 6 , like gratitude, rely on this, and are used in interventions with depressed patients to help them improve their mood while other, longer techniques are used to heal the evaluative sources of their depression. Focusing on evaluated wellbeing, even if it accounts for the lion's share of the causes of experienced wellbeing, obfuscates the existence of these independent sources of experienced wellbeing.

A final commendable feature of the subjective well-being definition is that it leaves the individual sovereign over their state of being. This is attractive to anybody who subscribes to liberal norms. Objective metrics of wellbeing are open to abuse by paternalistic or authoritarian governments that desire to dictate to their citizens. An extreme case would be any of the totalitarian regimes of the $20^{\text {th }}$ century, where citizen's wellbeing was defined in terms of how well society conformed to a utopian vision. In China, for example, the horrors of the Cultural Revolution were justified on the grounds that it was accelerating and deepening communism. More benignly, if a government pursues economic growth but does not deliver on more qualitative considerations like housing affordability and cultural policy, this won't show up in GDP figures but it will show up on subjective wellbeing variables. 
The liberalist merits of the SW-B definition can be presented more abstractly against competing hedonistic and objectivist theories. A hedonistic definition of well-being runs the risk of degenerating into a society that pursues pleasure and the absence of suffering at the expense of any kind of depth of thought or feeling. This possibility is eloquently explored in Aldous Huxley's Brave New World. In his arguably dystopic vision of the future, people take Soma (read: antidepressants) at the slightest hint of negative emotions, including mere thoughtfulness, and focus all their energies on sexual gratification and work. When one of the protagonists complains “but I don't want comfort. I want God, I want poetry, I want real danger, I want freedom, I want goodness. I want sin.” He is sent away to the Falklands least he destabilise Utopia. The protagonist's complaints would have more traction in policy circles if those circles were animated by a subjective definition of wellbeing.

The kind of thinking that lies behind classic objectivist theories of wellbeing are implicated in all of the horrors of historical totalitarianism and theocracy. Objective list theories tend, like Aristotelian eudaimonism, to specify well-being in terms of pursuing "the good". There is almost always a strong and clear normative dimension to these theories, which include things like Christian notions of Godliness, fascist notions of racial purity and even materialistic capitalism's occasional over-simplification of wealth as synonymous with wellbeing. If the good is what matters for wellbeing, then a government or ruling class has a moral imperative to bring its citizens into line with that good. Of course it is the ruling class that defines the good, and so there is a tendency in the context of objectivist states to be authoritarian. More recent incarnations of objectivist theories of wellbeing, notably the capabilities approach, especially as articulated by Nussbaum (2000), get around these problems by specifying fundamental liberal rights as inherent to the good or as universal values to be promoted.

The SW-B definition as an instrument of public policy arguably gets around these concerns not only by letting individuals decide whether they are well but also by leaving individuals free to decide what well means to them. In some articulations of the definition, it is further implied that individual wellbeing will depend on whether people are able to satisfy their preferences (their "ideals"). It follows from this that SW-B presupposes a liberal world order that leaves individuals free to pursue whatever normative code they wish. 


\section{Summary}

The field of SW-B research had to define itself against a backdrop of scepticism towards anything subjective and a centuries old body of wellbeing theory. In order to do this effectively, it opted for a fundamentally empirical approach that emphasised a very general, outcomes-based definition of wellbeing derived in no small part from the instruments used to measure that particular conception of wellbeing. This definition initially emphasised affective state - derived from experience sampling methods - and life satisfaction — derived from scale questionnaires. More recently, the definition of subjective well-being has also emphasised that subjective is defined in terms of whatever the respondent's ideals are. Even more recently, SW-B scholars have come to suspect a role for eudaimonic issues in SW-B, but have stopped short of a full blown engagement owing to what they perceive as a lack of effective measurement tools (OECD 2017, p. 32-33).

The merits of the SW-B definition of wellbeing are three fold. First, the very broad definition obviates against research being biased by theoretical priors. It places a strong and overdue emphasis on the empirical as opposed to theoretical investigation of wellbeing. Second, the discrimination between experienced and evaluated wellbeing makes clear the important distinction between these two aspects of wellbeing and allows their natures and interrelationships to be more accurately understood. Finally, the central position of the respondent in both the definition of subjective well-being and its assessment ensures a degree of commitment to liberal norms that are arguably important in the context of wellbeing policy. In the next chapter, I turn to consider criticisms of the SW-B paradigm, motivating a deeper engagement with the wider wellbeing literature. 


\section{Chapter 2}

\section{Theoretical Problems with Subjective Well- Being}

But I don't want comfort. I want God, I want poetry, I want real danger, I want freedom, I want goodness. I want sin.

- Aldous Huxley, Brave New World

\section{Introduction}

As discussed in the previous chapter, the paradigm of SW-B certainly has merits. However, it is also not without weaknesses. These are the subject of this chapter. I argue that while the definition and methodology of SW-B was acceptable within the field's original remit, it is inappropriate to applications in welfare economics and public policy. Once you leave the realm of SW-B scholarship with its relatively narrow questions and peculiar disciplinary and historical background you need to adopt a more sophisticated definition of "wellbeing" and more complex and incisive methods than life satisfaction scales. This requires engaging with all the interdisciplinary theory that SW-B scholarship has largely and deliberately avoided. It also requires then subjecting a fit-for-purpose theory of wellbeing to empirical tests designed to unearth the causal structure of that wellbeing. This is in contrast to the exploratory data analysis approach of SW-B scholarship to date. As Alexandrova (2017) has argued, the definition of well-being used in SW-B to date is acceptable for a field science, but the field is now relatively mature and needs to become a theory-driven science and ultimately a laboratory science. For this, deeper theorising about what wellbeing is and how it can be measured is required. Given the field has achieved increasing academic reputation in recent years, now is a perfect time to engage in this critical reflection. 
I split the weaknesses of SW-B scholarship into three groups: epistemic, theoretical and measurement. This chapter covers the epistemic and theoretical weaknesses, while chapter 10 discusses measurement issues.

Under the heading of epistemic concerns I discuss issues of circularity, the application of construct validation techniques in SW-B scholarship, and evidential subjectivism. SW-B is defined in terms of the instruments used to measure it. These are then justified on the grounds that they do indeed measure SW-B. This is circular and problematic from an epistemological point of view. One cannot logically arrive at conclusions about reality using this methodology, only conclusions about constructs. "Construct validation" exercises are required to exit circularity. However, the construct validation used in SW-B scholarship is arguably invalid in this regard. Construct validation as an exercise is itself only epistemologically sound if it begins with thorough theorising about its construct of interest. Owing to the field's history, SW-B scholarship avoids this crucial bit of initial theorising and its construct validation exercises are thus arguably ineffectual. SWB scholarship's approach is further undermined epistemologically by its adherence to evidential subjectivism. This is where both the measurement of a construct and the construct itself is subjectively determined. I canvass a range of further weaknesses in SW-B research associated with its disinclination to theorise that aren't strictly epistemological issues. First, I consider whether affect and life satisfaction are an appropriate conceptualisation of wellbeing for the contexts SWB is starting to press into. I explain how SW-B research sneaks a range of political and normative assumptions into its work, often inadvertently, under the guise of an empirical methodology and value-neutral definition of wellbeing. Second, I explore how insufficient philosophising in SW-B research leads to some outcomes that are of low marginal value because they merely rediscovers things we already know and/or overlooks deeper and more complex issues. Third, I discuss the difficulty of understanding how different aspects of a complex phenomenon like wellbeing interrelate and interact without thorough theorising. Finally, I note the tendency in SW-B research towards disjunctivitis, which is again a function of insufficient theorising about how different elements of wellbeing fit together.

The discussion in this and the next chapter ultimately builds to the claim that now is an appropriate juncture in the scholarship of wellbeing to take stock, posit an integrated theory of what wellbeing is and consider how it might be measured accurately and in a manner that 
aids causal identification. Presenting this theory and contemplating how it might be investigated empirically then becomes the subject of the rest of the dissertation.

\section{Epistemic Concerns}

\section{Circularity}

In conventional scientific method of the Popperian variety, research proceeds from hypothesis to test (Popper 1934/59). You begin with a theory and you attempt to find evidence refuting that theory. If it stands up to the scrutiny of tests designed to prove it false, you treat the theory as factually accurate until such time as falsifying evidence comes along. The purpose of this approach is to overcome the problem of induction. This problem concerns the difficulty of making inferences about a population from a sample of that population An intuitive statement of this problem is that if you observe swans a million times and every time they are white, you still can't be certain that somewhere in the world (like Australia) there isn't a swan that is black. All empirical knowledge is based on sampling and it may be the case that you simply haven't seen enough of the distribution. Falsification is a kind of solution to the problem of induction because you are not trying to prove something but rather to disprove it. You are not trying to demonstrate the truth of the statement that "all swans are white" but rather to find an exception. Until such an exception is discovered, we can treat the statement "all swans are white" as fact based on our existing sample of observations. We can continue to build a paradigm on the basis of our existing facts.

In important ways, the science of SW-B does not adhere to this approach. One of these ways is that SW-B scholarship did not first develop a theory of wellbeing, then measurement instruments that could access aspects of this theory so that they could be tested. Instead, it began by developing measurement instruments and collecting data. Only then did it begin to make theoretical conjectures about the nature of wellbeing, or in this case, SW-B. SW-B is defined basically in terms of the instruments that are used to measure it. Experience sampling methods were developed for measuring affect, and affect then became a component of subjective wellbeing. Life satisfaction scale questions were developed for measuring evaluated well-being, and then life satisfaction came to be part of the definition of SW-B. SW-B scholars are now considering incorporating meaning and purpose into SW-B, but are holding off until they develop measure of the concept (Stone and Mackie 2013, OECD 2017, 
Clark et al 2018). Again, the measure of the construct is preceding theory about what the construct actually is.

Circularity ensues when data collected using these instruments is then used to justify the instruments as accessing the construct that researchers are interested in. A positive result is inevitable. There is virtually no way that a question about life satisfaction could fail to capture life satisfaction. That doesn't mean that life satisfaction is wellbeing. What wellbeing is was never defined in the first place. The definition of the phenomenon of interest comes after something has been measured, and the phenomenon is then defined as whatever it is that is measured.

Another example might make this clearer. After SW-B was defined as some function of affect and life satisfaction, SW-B scholars noted that the two were not closely correlated (Diener et al 2006). From there, they posited that wellbeing is both experienced (affect) and evaluated (life satisfaction). However, there was never any theoretical justification of why affect and life satisfaction are both part of wellbeing. As noted in the previous chapter (in praise), this definition does not map neatly onto any definition in the philosophical, economic or health traditions. Even hedonistic conceptions, which are the closest analogy, typically emphasise experience or evaluation (Feldman 2002). Philosophers have previously argued that momentary affective states should not count towards wellbeing (Annas 2004, Sen 1999). Instead, we should focus on "emotional wellbeing", which is concerned with emotional disposition rather than emotional state. According to this argument, we would not say that someone who is "happy most of the time" is unwell simply because they're in an unusually bad mood (Haybron 2001).

The fundamental problem with this circularity is that SW-B research never actually posits a hypothesis about reality that is then subjected to tests. Theory comes after the test. It is logically impossible to arrive at a fact about reality using this method. Instead, one develops facts about constructs. This then motivates the exercise of "construct validation". Here again, SW-B scholarship runs into epistemic problems.

\section{Construct validity}

The primary means by which SW-B scholars justify their definition of wellbeing is by appeal to psychometric evidence, in particular construct validation exercises (Kaminitz 2018, p. 
433). "Constructs" in this context are "literally something that scientists 'construct' (put together in their own imagination) and which does not exist as an observable dimension of behaviour" (Nunnally and Bernstein 1994, p. 85). There are many things in psychology that cannot be observed objectively and thus require the development of a construct in order to be operationalised. Intelligence is a famous example. Construct validity refers to "the degree to which a test measures what it claims, or purports, to be measuring" (Cronbach and Meehl 1955). More specifically, construct validity refers to whether a "measure performs in the way theory would suggest with respect to the construct being measured" (OECD 2017, p. 49). In the case of SW-B, scholars claim that questions about, in particular, life satisfaction, do in fact measure wellbeing.

Alexandrova (2017, p. 131) describes the "implicit logic" of construct validation as follows:

A measure (M) of a construct $(\mathrm{C})$ is validated to the extent that $\mathrm{M}$ behaves in a way that respects three sources of evidence:

1. $\mathrm{M}$ is inspired by a plausible theory of $\mathrm{C}$

2. Subjects reveal $\mathrm{M}$ to track $\mathrm{C}$ through their questionnaire answering behaviour

3. Other knowledge about $\mathrm{C}$ is consistent with variations in values of $\mathrm{M}$ across contexts

Hedonic psychologists have certainly produced a great many studies that attempt to employ the logic outlined above to seemingly demonstrate the construct validity of SW-B. The OECD (2017) Guidelines on Measuring Subjective Wellbeing provides a good summary of the construct validity evidence that exists for life satisfaction and affect as measures of wellbeing. To wit:

Among individuals, higher incomes are associated with higher levels of life satisfaction and affect, and wealthier countries have higher average levels of both types of SW-B than poor countries (Sacks, Stevenson and Wolfers 2010). At the individual level, health status, social contact, education and being in a stable relationship with a partner are all associated with higher levels of life satisfaction (Dolan, Peasgood and White, 2008), while unemployment has a large negative effect on life satisfaction (Winkelmann and Winkelmann, 1998). Kahneman and Krueger (2006) report that intimate relations, socialising, relaxing, eating and praying are associated with high levels of net positive affect; conversely, 
commuting, working, childcare and housework are associated with low levels of net positive affect. Boarini et al. (2012) find that affect measures have the same broad sets of drivers as measures of life satisfaction, although the relative importance of some factors changes.

Further, it is clear that changes in SW-B - particularly life evaluations - that result from life events are neither trivial in magnitude, nor transient. Studies have shown that changes in income, becoming unemployed, and becoming disabled have a long-lasting impact on life satisfaction (e.g. Lucas 2007, Lucas et al 2003, Diener et al 2006). Although there can also be substantial individual differences in the extent to which people show resilience, or are able to adapt to, adversity over time. In the case of negative life experiences, Cummins et al. (2002) note that extreme adversity is expected to result in 'homeostatic defeat'-thus, life experiences such as chronic pain of arthritis or the stress of caring for a severely disabled family member at home can lead to stably low levels of SW-B. Similarly, Diener et al (2006) describe evidence of partial recovery from the impacts of widowhood, divorce and unemployment in the five years following these events, but SW-B still fails to return to the levels observed in the five years prior to these events. Thus, although there is evidence of partial adaptation to changes in life circumstances, adaptation is not complete, and the impact of these life events on evaluations is long-lasting.

It is worth noting that these studies all draw on panel data and do not use sources of exogenous variation or quasi-experimental methods to establish the causal claims they make. However, the weight of evidence points to SW-B measures moving broadly in the directions we would expect following events commonly associated with positive and negative effects on wellbeing such as divorce, unemployment and spinal injury.

Despite this evidence, the OECD Guidelines are ultimately very measured in their conclusions as to the validity of SW-B metrics. They argue that "an extensive body of evidence" accumulated over the last two decades "strongly supports the view that measures of both life evaluation and affect capture valid information". However, they note that this does not mean "that measures of SW-B are universally valid or devoid of limitations". They emphasise that SW-B measures should only be regarded as "fit for purpose" if "used with appropriate caveats" (p. 50). 
The OECD's reasons for hesitation all concern statistical matters. They note that SW-B measures have a relatively high noise-to-signal ratio of around 20:80-40:60. Things like the day of the weak, the season and the weather, among others, can all influence certain SW-B metrics. SW-B measures are also sensitive to survey content. For example, questions about the political state of the nation result in lower responses to subsequent life satisfaction questions. A recent paper (Husser and Fernandez 2018) found that simply adding "in your life" to the end of life satisfaction questions resulted in respondents giving higher scores. This implies that the words made people think more of the state of their own life rather than society in general. ${ }^{2}$ The OECD also notes that respondents vary in their response styles and how they interpret questions.

The OECD does not discuss more theoretical concerns with SW-B construct validation. Of note in this regard is Alexandrova's (2017) argument that construct validation in the context of SW-B does not use enough theory to support its implicit logic:

None of the conditions as they are currently implemented are strong enough to ensure validity...Indeed the philosophical heart of condition 1 [ $\mathrm{M}$ is inspired by a plausible theory of $\mathrm{C}$ ] is often enough replaced by an informal report of folk views or an unsystematic literature review. Instead of examining the nature of wellbeing of the relevant kind by building at least in outline a mid-level theory of it, the temptation is to canvass how this concept is understood by the relevant population and be done.

The list of validations referred to by the OECD makes this charge of "folk theorising" clear. Only the most straightforward postulates about wellbeing are used to validate the instrument - things like income effects and unemployment. Deeper issues like goal attainment, norms and preference satisfaction are overlooked, in part because of data limitations. This is a shallow engagement with what wellbeing is. There is little reference to the rich history of philosophical or psychological literature on the topic. This is especially problematic when the validation of measures using such simple theory is then taken to make the measures valid for more nuanced work, like cost-benefit analysis. This is what Alexandrova is getting at when she refers to the "wellbeing of the relevant kind". Wellbeing is an extremely rich concept with a great deal of political and sociological baggage. How we

\footnotetext{
${ }^{2} \mathrm{~A}$ curious corollary of this observation is that it suggests people think society is worse than it actually is. If on average people are more satisfied with their life than with society in general, then presumably they think others are more miserable than they actually are.
} 
understand it can vary from context to context. For example, orthodox economists tend to think of it in terms of the distribution of stuff (Agner 2015) while and social workers dealing with children think of it in terms of body weight and brain development (Waldfogel et al 2010).

Validating a construct using the broadest possible theoretical postulates makes sense if you are trying to carve out a niche amidst logical positivism, but is inappropriate when you're engaging in a broader socio-political exercise. In the context of such an exercise, outsourcing theory to statistics inadvertently robs normative issues of their significance by turning them into technical questions. Alexandrova explains well the dangers of this tendency in SW-B scholarship:

But it is less modest once we see its political context. Historians of psychological sciences have long noted the convenience of the methods of these sciences to the political order in which they arose and endure...psychologists, psychometricians, psychotherapists, and even psychoanalysts, have long played a crucial role in the management of individuals in liberal democracies. Their authority as advisors depends on their adoption of technical methods for handling questions that were not previously within the domain of science - what it means to be normal, intelligent, well-adjusted and so on. As Rose argues, this is how moral or prudential questions are turned into psychological ones. Similarly in our case, in undertaking the validation of measures of well-being, psychometrics puts itself forward as the arbiter of questions that are properly moral and political....Appeal to subjects' behaviour or their reports is a standard move. It makes validation procedures seemingly democratic and grounded in facts - and evidence-based too, so very convenient. Far from being modest and safe, this avoidance of philosophy and its replacement with a technical exercise in construct validation is epistemically wrong and morally dangerous. (ibid. p. 147)

Such arguments are echoed in Davies (2015), who focuses specifically on the socio-political implications of happiness studies and their potentially insidious consequences.

It is important to underline, as Alexandrova does, that "these questions cannot be resolved by checking more correlations". There needs to be "explicit deliberation of what counts as wellbeing to a given community". There are no resources in the implicit logic of construct validation to permit such an exercise. 


\section{Evidential subjectivism}

There is an additional epistemic problem for SW-B associated with the circularity of its definition: evidential subjectivism. Unlike other applications of operationalism, such as the measurement of intelligence, SW-B scholarship not only uses subjective measures of its construct of interest, it also uses a subjective definition of that construct. As Alexandrova (2017) explains:

Evidential subjectivism is a bet that not only is the object of measurement always a psychological state but, more importantly, the evidence used to validate a measure of this state must itself feed mainly from reports or behaviour of the relevant subjects in relation to this measure. Whatever philosophical evidence we might have about the nature of the psychological states relevant to well-being are reduced to observations of the behaviour of respondents to questionnaires (ibid. P. 130).

The principle epistemic problem with evidential subjectivism is that responses to SW-B questions across individuals do not necessarily elicit a measure of the same construct. We don't know how our respondents are defining the construct when they answer our questions. This makes developing a clear and unified definition of wellbeing using such questions difficult if not impossible. We fundamentally don't know what it is that we are measuring. We haven't defined it and developed measures for it. Instead we have started with subjective measurement elicited by asking people about our construct of interest. This approach cannot logically produce a clear hypothesis or a test of that hypothesis.

A related issue is that evidential subjectivism undermines our ability to develop better theory by admitting any and all conceptions of, among other things, life satisfaction. This undermines the role of theorists in refining the concepts under investigation. It gives too much credence to bad theories. To quote Alexandrova again:

Suppose the goal was to measure my happiness. It is certainly appropriate to check that my understanding of the related concepts (contentment, peace, elation, engagement, etc.) matches how the measurer understands these concepts. But it does not follow that I am the only authority on what happiness is. There are better and worse theories of happiness as we have seen, and this plain fact appears to be denied by evidential subjectivism, which in turn appears to be written into the very procedure of measure validation. P. 136 
In the next section, I explore the history of SW-B's operationalist methodology in psychology more generally. This makes it possible to pinpoint where SW-B crosses over from a domain where operationalism is appropriate to where it is not.

\section{Operationalism in SWB and psychology more generally}

The methodology of SWB research is grounded in the field's historical context. As discussed in the previous chapter, when SW-B scholarship emerged, psychology was dominated by logical positivism. SW-B scholarship could only carve itself a niche by focusing resolutely on empirics rather than theory. In consequence, it developed not only subjective measurement tools like experience sampling and life satisfaction scales, but also a subjective definition of wellbeing that left respondents free to decide what they meant by the term. Importantly, how respondents understood the term was only very rarely asked directly.

In a recent article, Kaminitz (2018) uncovers the history of the SW-B approach in psychology more generally, especially with regard to interpersonal comparisons of utility and satisfaction (IPCS). She notes that where difficulties associated with IPCS led economists to focus on the objectively observable metrics of real choices and money and thereby develop the doctrine of revealed preferences, psychologists instead opted for a different epistemic approach (Kaminitz 2018, p. 432):

An interesting starting point is the reaction of psychologists to logical positivism in the 1930s and 1940s, the period of intellectual history that saw economists embracing the sceptical approach to the question of scientific comparisons of utility between individuals. Significantly, positivistic influence led psychology along a completely different path. In particular, the work of S. S.

Stevens... actually opened the door to quantified scientific comparisons between individuals' inner worlds. The key to unlocking this door was Steven's (1946) seminal definition of measurement: "measurement, in the broadest sense, is defined as the assignment of numbers to objects or events according to rules." This is what philosophers of science would call a nominalist definition of measurement. For Stevens, methods of measurement are definitive of concepts; a view that stands in opposition to realism, which takes measurements to be methods of finding out about objective quantities that we can identify independently of measurement. Indeed, Steven's nominalism took the radical 
form of operationalism: the view that the meaning of a concept is fully specified by its method of measurement, implying that each measurement operation defines its own concept. The relevancy of this stance to our concerns is straightforward: scientists who hold to nominalism do not commit to measuring real entities-real life-satisfaction/happiness included; so from this perspective the impossibility of inter-personal comparisons of real quantities of satisfaction is obvious, but irrelevant.

Kaminitz's observations reveal the roots of the SW-B approach and also why it is problematic when used outside the disciplinary boundaries of SW-B scholarship. SW-B scholarship uses an operationalist epistemology rather than a realistic one: SW-B is defined by its measurement, as we have seen. This is in contrast to the other traditions investigating wellbeing. They require a concept of interest to be pinpointed in reality before metrics are developed to measure it. It was precisely the quagmire of theory these traditions had developed that SW-B wanted to avoid at its genesis. Kaminitz notes, as I did in the previous chapter, that psychology in general and SW-B scholarship in particular was able to make a great deal of progress by sidelining such complex theoretical issues:

Steven's definition was widely accepted within the psychological community and integrated into the basic psychological toolbox and textbooks of the second half of the twentieth century. It has opened the way for tremendous progress in the development of methods of measurement by putting to one side substantial philosophical questions pertaining to issues of ontology and epistemology. (ibid. p. 432-433).

Where this approach becomes problematic is when SW-B scholars move into other wellbeing domains, like public policy, and attempt to (often inadvertently) ride roughshod over other definitions of wellbeing that they find there. The argument used in favour of the SW-B approach in such cases is that it is grounded in evidence and value-neutral. But this is misleading. Due to their operationalist approach, what SW-B scholars have is an empirical understanding of $S W-B$, not wellbeing. They have an empirical understanding of affect and life satisfaction. It does not follow that this way of understanding wellbeing is appropriate for contexts other than SW-B scholarship. In other domains people are interested in a particular conception of wellbeing and justify this conception with reference, typically, to a range of political, ethical and contextual issues. 
When SW-B scholars, including happiness economists, attempt to supplant these existing definitions of wellbeing along with their justifications and replace them with the SW-B definition on the grounds that it is value neutral, they are in fact engaged in a sleight of hand. In these cases, the importation of an operationally defined concept of wellbeing amounts to sneaking in a variety of ethical and political assumptions regarding the nature of wellbeing under the guise of what appears to be a sterilised concept. ${ }^{3}$ The definition of wellbeing used in SW-B scholarship is in such cases presented as admirably theory-free, but it should in fact be criticised for being theory-avoidant. What is going on here is that SW-B scholars are inadvertently defining wellbeing in substantive ways for purposes to which that definition of wellbeing may be objectionable, and heading-off criticism but saying that their definition is entirely derived from observation. Alexandrova (2017, p. 93) provides a neat anecdotal explanation of why this is concerning:

The most serious charge is an importation into science of substantive views about the nature of well-being that those whose well-being is being studied may have good reasons to reject. This danger is real. When eminent economists including Nobel Prize winners advocate a measure of national well-being that takes into account only the average ratio of positive over negative emotions of the populace (Kahneman et al 2004b), the citizens can legitimately object if they take wellbeing to consist in more than that. Perhaps they believe that national well-being should also encompass compassion, kindness, and mutual trust of their community, the sustainability of their lifestyle, not to mention justice.

The items Alexandrova lists at the end of the above quote-compassion, kindness, trust, sustainability and justice - are all features of the long history of philosophical engagement with wellbeing. This philosophy is the study of what makes a life or some part of it good for an individual - the study of prudential value. As already discussed, SW-B scholarship had to put such considerations to one side to make progress in its domain, which is fair enough. But it is now bringing its view to other domains and failing to acknowledge, let alone engage with

\footnotetext{
${ }^{3}$ Davies (2015, p. 6) notes that this tendency has a long historical record going back at least to Bentham: Repeatedly, from the time of the French Revolution to the present (accelerating in the late $19^{\text {th }}$ century), a particular scientific utopia has been sold: core questions of morality and politics will be solvable with an adequate science of human feelings. How those feelings are scientifically classified will obviously vary. At times they are 'emotional', at other times 'neural', 'attitudinal' or 'physiological'. But a pattern emerges, nevertheless, in which a science of subjective feeling is offered as the ultimate way of working out how to act, both morally and politically.
} 
the greater complexity of wellbeing in the context of those domains. To explain why its definition of wellbeing is the appropriate one for exercises like cost-benefit analysis, SW-B scholarship must provide theoretical argument as to why its conceptualisation of wellbeing is superior to others in these new contexts. I discuss this issue in greater detail in the next section.

\section{Theoretical Concerns}

Is subjective well-being the kind of wellbeing we are interested in?

Alexandrova (ibid, p. 142) notes in her discussion of construct validation in SW-B research that "part of measure validation should be whether the measure captures a construct that is worth caring about". In this section, I take up this issue of whether life satisfaction and affect (and now meaning in life) are what we care about when we speak of wellbeing. My conclusions are mixed. On the one hand, all of these concepts have a long history in thinking about wellbeing. On the other hand, as they are used by SW-B scholars without a coherent theory of wellbeing, they are sometimes applied in problematic ways. Moreover, these items represent a kind of grab-bag of concepts that are relevant to wellbeing. They are in fact relevant, as is evidenced by all the construct validation work that has been done on them, but little attention has been paid to how these concepts fit together or what their relative importance might be. What work has been done is almost entirely psychometric: for example, checking correlations between affect and satisfaction. This can be misleading if we have no intuitive understanding of how and why these items might be related in complex ways. This becomes particularly problematic when it comes to understanding the causal structure of wellbeing.

Alexandrova argues that affect and life satisfaction aren't necessarily relevant aspects of wellbeing for people working in particular fields. For example, doctors studying the wellbeing of babies are mostly interested in physical indicators of wellbeing like birth weight. She consequently advocates for a variantist concept of wellbeing that can fluctuate according to the context in which it is being used. I am sympathetic to her argument. This dissertation is interested specifically in applications of wellbeing in economics and public policy. SW-B scholarship's definition of wellbeing is relevant to research questions in these fields and it is growing in influence. However, SW-B's definition also differs in marked ways from traditional conceptual frameworks in these fields. In particular, in happiness economics 
"wellbeing" is synonymous with utility (Van Praag and Frijters 1999), and in conventional economics utility comes from "goods" rather than life satisfaction and affect. Now "good" is obviously a much broader concept than affect and life satisfaction. The economic approach here echoes what philosophers in the analytical tradition call the study of prudential value or what makes a life "good-for" an individual. There are problems with the "prudential value" conception of wellbeing that I will discuss in the next chapter. SW-B scholarship, including its approach to definition, has actually been a welcome challenge to the philosophical tradition and has opened new areas and methods of inquiry. However, adopting the prudential value point of view for a moment can also illuminate how the SWB approach can lead to problematic outcomes.

Problems arise for SWB scholarship when it moves from merely studying affect and life satisfaction to suggesting that these are "good-for" people. Some examples from SW-B's policy advocacy work will help illustrate. Easterlin's earliest paper (1974), which arguably kicked-off happiness economics, was entitled "does economic growth improve the human lot?" His measure of the human lot, that is, of prudential value, was life satisfaction. This would be disagreeable for someone for whom the human lot is a function of justice, the realisation of the kingdom of heaven on earth, goal pursuit, survival, perfection or truth, among other possibilities. I don't think that life satisfaction is a worse definition of the good than any of these options (quite the opposite in fact), but it is worth underlining that life satisfaction is merely one among many competing options. This is why utilising the SWB definition of wellbeing in policy advocacy without admitting its normative claims amounts to trying to sneak moral and political values in under the guise of a value neutral, "empirical" definition.

To see why these alternate specifications of what is good-for individuals are important, consider the implications of defining the prudential good as life satisfaction for matters of distribution. The claim that what ultimately matters is happiness or wellbeing and thus that justice amounts to ensuring an equal distribution of these things in society is called "welfarism". Advocacy for welfarism is implicit in calls for SW-B metrics to be used in costbenefit analysis (Fujiwara and Dolan 2016). One of the most prominent arguments against welfarism was made by Amartya Sen (1999). He noted that people get used to their circumstances - they have "adaptive preferences". As such, a downtrodden, impoverished wife in rural India can have a similar level of life satisfaction to a wealthy but ultimately frustrated businesswoman in New York. Precisely such a situation is observed in the 
literature on "happy peasants" and "frustrated millionaires" (Graham and Pettinato 2002). If the only thing that is prudentially good is life satisfaction, then a social planner considering how to allocate society's resources should be indifferent between these two individuals. This outcome strikes most people as perverse. It seems that the prudential good also has something to do with living in a just society or getting your justly deserved slice of the collective pie. That would explain why we think the happy peasant deserves redistribution from the millionaire even though the millionaire is less happy. It is worth pointing out that Easterlin's research was not aimed at demonstrating that life satisfaction is the prudential good. Rather, it was aimed at critiquing the prevailing view that economic growth is the prudential good. In this it was certainly an important endeavour.

Another example: Clark et al (2018) advocate for a range of schools policies on the basis of their effect on the emotional wellbeing of children. They focus on emotional well-being because of a lack of data on other dimensions. This is fine, but as I discussed in Fabian (2018), they do not consider whether other dimensions of wellbeing might be harmed by an excessive emphasis on emotional wellbeing. Consider that learning many important things, even when that learning is broadly enjoyable, can involve a great deal of negative emotion, like stress, low self-esteem and boredom. We learn them anyway for payoffs in terms of other dimensions of well-being, like identity, employability or purpose. So if we focus on the emotional wellbeing of children without considering longer term eudaimonic and other payoffs, we may well do those children a disservice. Stone and Mackie (2013, p. 16) make a similar point:

The terms used to describe SWB have often been ambiguously applied, which has muddled discussion and possibly slowed progress in the field. For example, the term "happiness" has been used to refer to momentary assessments of affect as well as to overall life evaluations. This absence of precision precludes understanding of the complexities known to coexist. For example, a person who is engaged in stressful or difficult activities, such as working towards an education or job promotion, may find substantial meaning or satisfaction with life overall; a person who is generally suffering or lacking hope may experience temporary reprieve in an enjoyable moment 
These examples illustrate why picking indicators of wellbeing from a grab bag rather than considering a more holistic model of what is "good-for" an individual can lead to problematic inferences.

\section{The need for philosophy}

Philosophy complements science in two main ways. First, by cleaning up existing empirical findings and organising them into coherent bodies of knowledge (see below on disjunctivitis). And second, by using deductive reasoning on inductively established facts to derive new hypotheses for empirical testing. In a sense, philosophy runs ahead of contemporary empirical research to scout the forward terrain. When philosophy is doing its job, empiricists should be able to draw on rich, clearly articulated theoretical work when conducting innovative research. Poorer outcomes emerge when philosophy is not doing its job, or when theorists are not paying attention to the work that has been done, or when empiricists are simply dismissive of anything theoretical as unscientific. In such cases, empirical work has a tendency to investigate things we already know, and to overlook deeper or more complex issues and thus test shallow hypotheses. In such cases, the marginal value of empirical work is low. Institutional settings in contemporary universities arguably have more to do with this than anything peculiar to wellbeing scholarship itself. Nonetheless, these issues are pernicious in this field, as the following examples indicate.

\section{Rediscovering what we already know}

A prominent example of missing something we already know is the recent rediscovery of the eudaimonic dimension of wellbeing by SW-B scholars. The distinction between hedonic and eudaimonic happiness goes back to antiquity. It features in the writings of Aristotle, the epicureans and the stoics. If anything, it is the hedonic dimension of wellbeing that disappears during the medieval period, only returning with Bentham and the utilitarians. The British fixation with the hedonic dimension of happiness puzzled continental philosophers of wellbeing, notably Nietzsche (1889/1990, p. 33), who mocked "man does not strive for happiness; only the Englishman does that". The eudaimonic dimension of wellbeing was prominent in Anglophone philosophy faculties in the second half of the $20^{\text {th }}$ century (Kraut 1989, Norton 1976, Griffin 1986) and has featured prominently as part of the recent revival 
of virtue ethics therein. Against this background, it is a little odd that eudaimonic aspects of wellbeing only start to figure in the SW-B literature in the late $2000 \mathrm{~s}$, owing at least in part to critiques from eudaimonic psychologists about equating wellbeing with happiness (Deci and Ryan 2006, Waterman 2008, Kashdan et al 2008).

That said, the application of the SW-B paradigm to eudaimonic issues has yielded some innovative new insights. For example, Dolan (2014) has used a simplified account of eudaimonia that emphasises the distinction between pleasurable and purposeful activities to explore eudaimonia from a behavioural perspective. Graham (2017) combines hedonic and eudaimonic perspectives on SWB to provide novel insights into economic behaviour and contemporary sociological trends in the United States. There is certainly a lot of value in integrating the two literatures.

Broadly speaking however, the response of SW-B scholars to the necessity of incorporating eudaimonia into how they think about wellbeing has committed precisely the mistakes of that incorporating other perspectives should help eliminate. Namely, there is a rush to develop cheap and simple measures of eudaimonia so that the strictly empirical approach can continue. There is little attempt to understand eudaimonia in a sophisticated way by engaging with it theoretically before moving to the development of empirical instruments for measuring it. It is consequently described in shallow terms in the SW-B literature as "meaning in life" or "good psychological functioning" (OECD 2017, Clark et al 2018). Overlooking the need to theorise at the outset will ultimately undermine research into eudaimonic wellbeing by SW-B scholars down the track.

It is worth noting that Daniel Kahneman, one of the progenitors of hedonic psychology and the field of SW-B research, has addressed similar critiques in the past (Kahneman 2010). In response to an article by Rakow on the "potential value of sourcing ideas from other disciplines or from earlier periods of time”, Kahneman said:

I think that the assertion in the last paragraph that "What I hope to have shown is how decision research in the late $20^{\text {th }}$ century could have benefitted from a close reading of Knight's Risk, Uncertainty and Profit, particularly as a source of useful hypotheses to pursue" makes no sense at all. This is not how science is done...Science is essentially a conversation in which people respond to what others have most recently said, or to the ideas that are currently dominant. Ideas that change the direction of the conversation are new because they are new in the 
conversation — not because no one has had them before.... Reading Knight would not have helped us at all — we would not have recognized that what he said meant the same thing as what we said...I don't think many psychologists draw their hypotheses from Plato or Montaigne, though these authors certainly said many things that sound similar to ideas that people proudly publish in Psychological Science.

It is fair for Kahneman to bring up the matter of scientific "conversation". As I have repeatedly emphasised, early SW-B research had a particular context—it's conversation, if you will - that necessitated its particular approach. However, SWB is now mature and breaking into a much wider conversation: the wellbeing conversation. It is hamstrung in its ability to contribute effectively to this conversation by features of its traditional approach, namely an absence of theory. It should not contaminate the wider conversation with this weakness. Instead, it should borrow the strengths of the wider conversation, like a deeper theoretical understanding of eudaimonia and wellbeing more generally, and use these to shore up its own weaknesses. Kahneman might be right that empirically, wide, interdisciplinary literature reviews are not how science is done. That doesn't mean it isn't how science should be done. Such an approach would result in much less duplication and wasted effort, provide clearer and more significant hypotheses to test, give layered answers and help to guide efficient data collection efforts.

\section{Overlooking deeper and more complex issues}

Good theory helps empiricists to understand their phenomena of interest holistically so that they can measure and test at the forward edge of our knowledge. Poor integration of theory into empirical research can lead to complexities being obscured. Empirical work then proceeds at a distance from the frontiers of knowledge.

The limited integration of preference theory into SWB research is illustrative. Almost no research in SW-B has incorporated preferences. This is despite a strong emphasis on the role of preference-satisfaction in promoting wellbeing in theory from philosophy, economics and psychology. Consequently, changes in SW-B are largely framed in terms of changes in circumstances rather than preferences and the role of preferences is left under-investigated. A prominent example is the long-running study of the effect of income on life satisfaction. Intuitively, the role money plays in your life satisfaction depends on what you want to spend 
your money on. If you are an ascetic with strong preferences around abstinence, money will have little effect on your life satisfaction. On the other hand, if you have a genuine love of fast and fancy cars, even a substantial income will be insufficient for you to be satisfied with your life. Furthermore, how your preferences change as you become richer or poorer, age and change your perspective on life will affect how rapidly your life satisfaction adapts to those income changes.

The ongoing debate around the life satisfaction effects of absolute and relative income seems somewhat facile in this context. Much of this research is of a very high calibre (for example: Clark et al 2008b, Stephenson and Wolfers 2013, Clark and Senik 2014a). However, because it doesn't take account of preferences it is unable to penetrate surface level phenomena to grasp the deeper causal structure of wellbeing. For example, one of the most striking findings in this field in recent years is Easterlin et al's (2017) observation that China's dramatic income growth over the past three decades has translated into little improvement in average life satisfaction scores. There are measurement issues to be concerned about, but leaving those to one side, Easterlin et al's findings support the hypothesis that absolute increases in income have a limited effect on SW-B due to adaptation to improved circumstances. In particular, income growth for poor Chinese workers has typically involved migration to cities where the upper end of their reference group changes from village leaders to urban business elites who are much wealthier. Migrant workers might consequently undervalue their improved circumstances as they now see how much better they could be.

These are important and valid hypotheses about the dynamics of wellbeing, but they can't be effectively investigated without considering the preferences of migrant workers. For the lack of change in life satisfaction to be driven by adaptation to income and reference group effects it would have to be the case that migrant workers develop stronger preferences for income after migrating to cities. It is possible that the limited change in life satisfaction is driven by other factors. For example, migrant workers may have static preferences for air quality that are not satisfied in China's urban centres ( $\mathrm{Li}$ et al 2018). Or they might have preferences for political enfranchisement that are both deepened and left unsatisfied by the poor treatment migrant workers receive under the prevailing hukou system of household registration (Liu et al 2017). In both cases, positive income effects from migration would actually be offsetting the negative impacts of migration on other preference domains. 
Some of the issues outlined above could be resolved using experimental or quasiexperimental methods. These would overcome the causal identification problems associated with naturalistic evidence. However, even in such cases, issues concerning preferences and causation could remain. If it were demonstrated through a quasi-experimental methodology that income increases satisfaction this might still leave open whether preferences were a part of this causal channel. Furthermore, this result would reflect an average effect of incomethe impact will differ by respondent according to their preference for discretionary spending and financial security. As preferences are fundamentally heterogeneous (and dynamic) across respondents, leaving them unaccounted for will limit the insights provided by any study of wellbeing performed at the aggregate level.

Solving this problem is not a matter of more sophisticated statistical techniques, though they might have a role to play. Nor is it strictly a function of having richer data. Even with better data, empiricists unequipped with a thorough understanding of how preferences are involved in wellbeing and its dynamics would struggle to ask the right questions. They would thus continue to operate in the shallows rather than the depths. What is missing here is a clear theoretical explication of the role of preferences in wellbeing dynamics that can be used to guide empirical research.

\section{Interplay of wellbeing dimensions}

The discussion of income effects above alludes to a more general reason why theory is critical to the study of wellbeing: understanding the causal channels between different dimensions of a complex phenomenon like wellbeing is very difficult without theory. Wellbeing transcends disciplines and fields of inquiry. It ranges across philosophy and all of the social sciences and takes in topics from the nature of pleasure to how humans create values and sustain meaning in normative communities. Studying the causal and dynamic structure of wellbeing requires some theoretical explication of how all these different literatures and aspects of wellbeing fit together. Avoiding this difficult work is liable to lead researchers to overlook mediating factors in wellbeing causation and deep determinants of surface-level phenomena. They may miss how different aspects of wellbeing fit together in causal ways. Statistical analysis has difficulty overcoming these issues without building on periodic theoretical analysis. This comes back to philosophy's role in cleaning up and organising existing empirical knowledge. 
An example will help illustrate. As mentioned earlier, there is presently a groundswell of interest among SW-B scholars in the eudaimonic dimensions of wellbeing. As per standard practice in the field, the first step in better integrating eudaimonia into SWB has been to find metrics that track it. Several such metrics have been considered, notably Huppert and So's Flourishing Scale and Diener and Biswas-Diener's Psychological Well-Being Scale. Existing metrics in the eudaimonic wellbeing field, such as Ryff's five-factor model of psychological wellbeing and the metrics used by self-determination theory have received less attention because they are typically relatively more laborious instruments that are suboptimal for the kind of aggregate-level and policy-oriented research SW-B scholars are most interested in conducting. The new metrics have been used to assess whether eudaimonic wellbeing is distinct from affect and life satisfaction. It seems to be. Clark and Senik (2011) report a correlation between life satisfaction and four different aspects of eudaimonic wellbeing of between 0.25 and 0.29. Diener et al (2009) report a correlation of 0.62 between their psychological well-being scale, which is supposed to reflect eudaimonic aspects of wellbeing, and life satisfaction scale responses. The correlation between their psychological wellbeing scale and measures of positive and negative affect is 0.62 and 0.51 respectively. Huppert and So (2013) found a correlation of 0.34 between "flourishing"-which takes in aspects of eudaimonic wellbeing — and life satisfaction in European social survey data.

These correlation statistics help to establish eudaimonic wellbeing as an important object of inquiry independent from affect and life satisfaction, but they do not enlighten us about the interrelationships between eudaimonia and these other aspects of SW-B. We can't tell, for example, whether eudaimonia correlates with life satisfaction because one causes the other, because there is two-way causation, or because people who are high in one just tend to coincidentally be high in the other. Now of course it is early days in this research project, and SW-B scholars might reasonably respond to this point by saying that they will get round to investigating such interrelationships. However, this project may well be futile without some preparatory theoretical deliberation about how we might reasonably expect eudaimonia to interplay with other aspects of wellbeing. Statistical analysis of this relationship would struggle to pinpoint its object unless we first consider what the relationship between these different aspects of well-being is like. We can then design an empirical instrument specifically to test this hypothesis.

To illustrate, consider the fact that nihilism (the belief that the world and existence is meaningless) only affects some people, and potentially only under certain social 
circumstances (its historical high tide was in the lead up to World War II in Europe). Nihilists escape their affliction, according to existential philosophy (De Beauvoir 1947/2002/2002) and terror management theory (Shaver and Mikulincer 2012), by creating meaning subjectively and comporting themselves towards self-concordant normative communities. Now consider that many people are insulated against developing nihilistic feelings in the first place by being raised in and socialised into normative communities. Examples include religious and village communities with clear value systems, rituals, and a shared symbolic language. The end result of either a nihilist building a normative community or a typical individual being born into one that works for them is life satisfaction. Now a statistical analysis at the aggregate level may well pick up that there is a correlation between "relatedness" and life satisfaction, and hence between eudaimonia and life satisfaction. It may even be able to establish this as a causal relationship using some quasi-experimental methodology. However, it will struggle to identify whether the high life satisfaction and relatedness scores of an individual are the result of them successfully overcoming nihilism or simply never having been afflicted with it in the first place. Moreover, such analysis will have a tendency to see nihilism as a poor predictor of satisfaction compared to relatedness, even though it is nihilism that is the primary agent. It is nihilism that causes the negative effect on life satisfaction; relatedness is a cure or insulator. Of course, if the researchers are savvy and able to ask the right questions of their data they can identify these interrelationships and nuances (or prove this theoretical explication false), but to become savvy in the first place they must adequately theorise.

\section{Disjunctivitis}

Insufficient theorising can lead to empirical researchers operating in silos rather than working together in cooperative enterprise. The result will be internally inconsistent bodies of knowledge. Antonakis (2017) calls this “disjunctivitis". Instead of advancing in paradigmatic fashion, researchers each take little steps in different directions. The research that gets done is fragmented, oftentimes incoherent as a body of work, and does not help science advance in a cohesive fashion. So for example, we have hedonic psychologists making a range of breakthroughs in mood management through techniques like gratitude and savouring. Meanwhile, eudaimonic psychologists make breakthroughs in how nourishing the basic psychological needs of relatedness, competence and autonomy can cure depression and 
anxiety. But we have little work on how these two domains of wellbeing interact on their relative contributions to an overarching construct like life satisfaction. In addition, other areas of SW-B research, like the philosophy of wellbeing or happiness economics, proceed with little reference to either of these psychological literatures (with exceptions of course).

Disjunctivitis finds its roots in institutional settings in contemporary universities. Most universities are organised by discipline and faculty are under pressure from their deans to publish in top disciplinary journals rather than field journals. This inevitably leads to siloing even in interdisciplinary fields like wellbeing. Faculty are also under tremendous pressure to publish, and typically to publish papers rather than monographs. This encourages the production of isolated works rather than the development of broadly-conceived, internally consistent bodies of knowledge. Finally, faculty are under pressure to be cited, which encourages them to invent new theory that they can stamp with their personal brand rather than simply building on or extending existing paradigms.

Nonetheless, disjunctivitis is also a function of an overly empirical approach to research and a disinclination to theorise. This mindset means that a researcher rarely needs to go beyond the boundaries of their own discipline to find enough theory to motivate an empirical exercise. Researchers consequently stop too often at those boundaries when they would find in other disciplines the material required to produce a richer, deeper theory to explore in their empirical work.

In addition, empirical work tends to be incremental in nature. It is averse to starting with a grand theory. Indeed, grand theories can be harmful to empirical research because they make hypotheses too complex to neatly test. This discourages empirical researchers from regularly taking stock of their field and bringing it into internal coherence. A manifestation of this is the contemporary tendency in empirical disciplines (which is increasingly all disciplines) to produce handbooks or other edited volumes where scholars in a field each contribute a chapter on what they are doing. This is in contrast to a smaller number of authors surveying the field and attempting to collect its findings into a unified theory within a single monograph. My contention in this dissertation is precisely that such a unifying project is overdue in the field of SW-B, or would at least be beneficial. 


\section{The way forward}

Alexandrova neatly sums up the weaknesses of contemporary SW-B research in the following passage:

By sticking to a resolutely anti-realist metaphysics the psychometric approach wrongly outsources to statistics what is essentially a theoretical problem: What must well-being be like, as a causal system, for questionnaires to detect it? (ibid, p. 148)

These weaknesses can be traced back to the implicit logic of construct validation, as I have explained earlier. In the concluding passages of her book A Philosophy for the Science of Wellbeing, Alexandrova (ibid. p. 150) advocates for a "Better Implicit Logic" to inform construct validity in wellbeing research (emphasis added to differentiate from the basic implicit logic quoted above):

A measure $\mathrm{M}$ of construct $\mathrm{C}$ can be considered validated to the extent that $\mathrm{M}$ behaves in a way that respects three sources of evidence:

1. $\mathrm{M}$ is inspired by a plausible theory of $\mathrm{C}$. This theory should be articulated as fully as possible and defended against alternatives.

2. $\mathrm{M}$ is shown to track $\mathrm{C}$ as $\mathrm{C}$ is understood and endorsed by the subjects to whom $C$ is applied.

3. Other knowledge about $\mathrm{C}$ is consistent with variations in values of $\mathrm{M}$ across contexts. This knowledge should encompass the normative significance of $C$, including moral and political contexts of the use of $C$.

In this dissertation, I attempt to fulfil condition (1). I elucidate a plausible theory of wellbeing that is appropriate to a range of domains, but especially to economics and public policy. I then consider whether contemporary SW-B metrics track this kind of wellbeing with sufficient accuracy for the purposes of wellbeing researchers, thereby making a start on (2). I find them somewhat lacking and consequently propose extensions to these metrics as a first step to developing better means of measuring wellbeing. 


\section{Chapter 3}

\section{The Analytical Philosophy of Wellbeing}

It is vain to talk of the interest of the community without understanding what is the interest of the individual.

- Jeremy Bentham, An Introduction to the Principles of Morals and Legislation

I begin the development of the production function theory of wellbeing from the analytical philosophy literature. There are two reasons for this. The first is that analytical philosophy is very attentive to issues of categorisation and delineation. Discussing the analytical literature allows the question of "what is wellbeing" to be made quickly tractable by outlining particular schools of thought. Explaining these schools and their strengths and weaknesses provides a picture of the landscape of wellbeing theory. The details can then be filled in. The second is that the analytical philosophy of wellbeing takes as its object of inquiry the prudential good. This is what is "good for" someone. The most salient critique of SW-B's recent forays into policy and welfare economics is that it pays insufficient regard to normative issues pertaining to the prudential good. In contrast, extensive theorising regarding the prudential good underpins the present paradigm in welfare economics. Commencing the development of a theory of SWB with analytical perspective gives due regard to the centrality of these issues to a holistic theory of SWB.

This chapter makes two principle claims. First, that recent cycles of debate between the three most prominent classes of wellbeing theories in analytical philosophy-hedonistic, subjectivist and objectivist - have resulted in a great deal of convergence between those different theories. Increasingly, advocates of one or other theory are only able to respond to critiques of their position by, in a sense, subsuming their interlocutor's perspective. This 
trend suggests that there might be value in giving greater thought and consideration to the similarities between wellbeing theories rather than focusing almost exclusively on their differences.

This brings us to the second claim, which is that adopting a practical perspective on wellbeing that considers the process by which wellbeing is attained rather than simply what it is reveals that these theories are more interdependent than their advocates might presume. Furthermore, it reveals that self-actualisation is critical to the acquisition of wellbeing regardless of which theory of wellbeing you are employing. Moreover, themes from all three classes of wellbeing theory are required to understand how self-actualisation works to promote wellbeing. I advance a particular model of self-actualisation in this dissertation, namely the coalescence of being, which is explained in detail in chapter 9.

The chapter is structured as follows. First, I present again Parfit's (1984) tripartite classification of wellbeing theories and show how recent articulations of each of these theoretical positions have involved adopting some ideas from the other theories. I argue that an integrated theory of wellbeing is thus worth pondering. In the second part of the chapter I sketch such an integrated theory. I call it the production function theory of wellbeing. Wellbeing in this theory is a function of hedonia, eudaimonia and despair, which refer to whether life is pleasant, fulfilling and valuable, respectively. In the third part of the chapter, I discuss the dynamics and praxis of wellbeing. This discussion reveals the importance of selfactualisation to the attainment of wellbeing. It also reveals a degree of interdependence between the major wellbeing theories.

Before moving on, a brief clarifying statement is required. Haybron (2011) helpfully distinguishes two different ways the term "happiness" is used in the scholarly literature and by the general public. He calls these "happiness in the psychological sense" and "happiness in the wellbeing sense". The psychological sense refers to the state of mind of happiness. This sense of happiness is most commonly employed, unsurprisingly, by psychologists, notably those working in the field of hedonic psychology. The wellbeing sense refers to what it means for a life to "go well" for an individual or what is "good-for" that individual. Another way of saying this would be happiness in the prudential sense. There is a strong normative dimension to happiness in the well-being sense. This sense is most common among philosophers. This dissertation is about well-being and thus happiness in the well- 
being sense. In some of the older philosophical literature, happiness is used instead of wellbeing in a way that appears to mean well-being in the prudential good sense. In such cases, I take the authors to be referring to wellbeing and use that word instead. Ultimately, I argue that Haybron's two senses of happiness are more interrelated than some presently believe. Furthermore, I argue that understanding wellbeing as what is "good-for" an individual invites confusion because people achieve wellbeing by pursuing their good. As such, the production function theory of wellbeing that I develop is hard to describe as either a theory of happiness in the psychological sense (i.e. a state of mind) or a theory of wellbeing as the prudential good. It is nonetheless a theory of wellbeing.

\section{Three classes of wellbeing theory}

Parfit (1984) partitioned wellbeing theories into three classes: subjectivist, objectivist and hedonistic. Hedonistic theories define wellbeing as pleasure (Gregory 2015). The most common subjectivist theory is desire- or preference-satisfaction (Heathwood 2015). More recently, some philosophers have defended a life satisfaction subjectivist theory, where the "individual is happy if they say they are satisfied with their life" (Vitrano 2013). Objectivist theories establish a universal standard by which to judge whether a life is happy (Fletcher 2015). There are two prominent traditions herein, one philosophical and the other psychological. Both distinguish themselves by their use of the term "eudaimonia". Among philosophers in the Aristotelian tradition, eudaimonia is the wellbeing that attends living virtuously in accordance with reason. More recently, some clinical psychologists working on wellbeing have distinguished their work from that of the so-called hedonic psychologists by characterizing it as promoting a eudaimonic view of wellbeing, meaning to live well, rather than a hedonic view, meaning to have a high degree of positive emotion (Deci \& Ryan 2006; Ryan et al. 2008). These clinical psychologists argue that there is empirical evidence that individuals whose lives nourish their three basic psychological needs for autonomy, competence and relatedness demonstrate eudaimonic wellbeing, whereas those whose lives do not nourish these needs suffer from depression, anxiety and other ailments. As such, the 
basic psychological needs provide an objective standard for judging someone's wellbeing. ${ }^{4} \mathrm{I}$ discuss these eudaimonic theories in greater detail in chapter 7.

Some theorists are increasingly moving away from Parfit's tripartite classification (see, for example, Woodard 2012). However, they are typically not moving towards an integrated theory, but rather towards a more fine-grained taxonomy. Here I want to take the opposite approach. Analysing the similarities between wellbeing theories rather than their distinctions provides a range of important insights. In the rest of this section, I explore recent articulations of the hedonistic, subjectivist and objectivist perspectives on wellbeing. I argue that recent iterations of the debate between these different perspectives have seen their distinctions reduced. The theories are now increasingly borrowing from each other.

\section{Hedonism}

Prominent hedonistic theories in history are those of the epicureans and the classical utilitarians. The most prominent recent entry into this class of theories is Feldman's (2002) attitudinal hedonism. Attitudinal hedonism is distinct from sensual hedonism, which concerns only feelings, specifically pleasure and pain. In contrast, attitudinal hedonism is concerned with attitudes, especially enjoyment, which Feldman argues is phenomenologically not a feeling. He argues that attitudinal hedonism "is a mode of consciousness", and considers it robust to many classical critiques of hedonism. For example, attitudinal hedonism can explain why the stoic is well in a way that sensual hedonism cannot. Stoics want peace and do not seek sensory pleasure. Thus on a sensory-hedonist account, they cannot possibly be well. Intuitively though, we feel that the stoic acquiring what she wants brings her wellbeing, so this account seems flawed. Attitudinal hedonism is robust to this critique, because while the stoic does not experience much pleasure, her life nonetheless goes well (ibid, p. 7).

\footnotetext{
${ }^{4}$ There is another variety of objectivist theory that I omit to discuss herein due to space constraints. This is the model of wellbeing one finds in quality of life and other medical research, where the researcher sets up a list of criteria that define high quality of life (see, for example, McClimans \& Browne 2011). An example is saying that a deaf person is fundamentally not capable of the same degree of wellbeing as a person who can listen to Mozart and talk with perfect elocution, regardless of how happy that deaf person claims to be. These metrics are designed for practical purposes and aren't fully fledged theories of wellbeing, so I omit them.
} 
Feldman also claims that attitudinal hedonism can withstand normative critiques. However, in order to do so, Feldman's attitudinal hedonism must essentially subsume the tropes of objectivist theories, and his theory thereby is no longer a purely hedonistic theory. There are three critiques that Feldman takes up. The first is that someone living a pleasant but deceived life is considered to have just as valuable a life according to hedonism as someone who experiences as much pleasure but is not deceived, and this seems wrong. The second is that hedonism does not differentiate between worthless and worthwhile pleasures - what Crisp (2006) calls the "worthless swine" criticism in reference to Aristotle. ${ }^{5}$ The final critique is that while hedonism might give us an account of what sort of life is worth living, it does not give us an account of what sort of world is worth creating.

In each case, Feldman responds by expanding his theory. First, he suggests that pleasure could be accorded more weight when it is drawn from a true state of affairs. Second, he suggests that pleasures from certain sources could be weighted to take into consideration the worthiness of those sources of pleasure. He calls this "dessert-adjusted intrinsic attitudinal hedonism". Finally, he suggests that we also weight pleasure to capture the worthiness of the subject receiving the pleasure. In this way, hedonism can answer the third critique by saying that the value of a world is the sum of the double dessert adjusted values of the intrinsic attitudinal pleasures enjoyed and pains suffered in that world.

Feldman's three extensions to attitudinal hedonism would seem to subsume the core arguments of the objectivist perspective on wellbeing in order to become robust to them. $\mathrm{He}$ says (p. 17):

Such things as excessive or deficient prior receipt, legal or moral 'rights' to pleasure, hard work, virtue and vice etc. probably influence the extent to which someone deserves some pleasure.

The argument of the objectivist perspective, especially eudaimonism, is precisely that these sorts of questions, such as "what is vice?" and "who is virtuous?", cannot be usefully answered with a hedonistic conception of wellbeing in the wellbeing sense. Feldman's

\footnotetext{
${ }^{5}$ J.S. Mill (1863) was responding to similar criticisms of utilitarian hedonism when he attempted to distinguish between the higher and lower pleasures.
} 
hedonism escapes these critiques only by, in a sense, admitting them. He maintains the core claim of hedonistic theories of prudential wellbeing, namely that what is "good-for" an individual is pleasure. But he then augments his notion of pleasure with objective principles about what sort of pleasure is "good for" an individual.

An arguably more telling critique of hedonism comes from Haybron (2001). He argues that hedonism "does little more than skim the phenomenal surface off our emotional states" while missing the deeper character of wellbeing. ${ }^{6}$ His arguments refer specifically to happiness, but they readily adaptable to hedonistic theories of wellbeing as well. This deeper character includes the fact that wellbeing is dispositional. A well person might be suffering right now, but we would still say that they are "well in general". We would also predict them to be well in the future. These are clearly not matters of present pleasure, nor can they be adequately described by the notion of a positive attitude.

Despite these shortcomings, it is important to rescue from the hedonistic tradition the idea that pleasure matters to wellbeing. The eudaemonist literature seems to deny this at times, or at least heavily marginalizes the importance of hedonia (Annas 2004). It is possible for pleasure and pain to relate directly to wellbeing. For example, sciatic pain made me irritable and impatient, undermining my hedonic wellbeing. Being unusually irritable and impatient in turn affected my sense of self, undermining my eudaimonic wellbeing. During episodes of sciatic pain, I would say that I am unwell, and one of my primary goals in life is to be rid of my sciatic pain and the injury that causes it. It thus undermines my wellbeing on a life- and desire-satisfaction account as well. Haybron's (2001) point that happiness and wellbeing are more than the phenomenal surface of our emotional states is a good one, but it doesn't mean that the phenomenal surface isn't nonetheless an important aspect of wellbeing.

\footnotetext{
${ }^{6}$ This critique seems particularly powerful when aimed at some of the early hedonic psychology definitions of subjective wellbeing, notably Kahneman's “objective wellbeing”, which essentially involved adding up someone's pleasure score over (moment-by-moment) time by taking an integral (Kahneman 1999).
} 


\section{Subjectivist theories}

One of the most well-known subjectivist theories of wellbeing is Sumner's (1999) life satisfaction or "authentic happiness" theory. ${ }^{7}$ He provides a summary statement in the following passage (p. 172):

[Happiness], we have found, can be equated with life satisfaction, which has both an affective component (experiencing the conditions of your life as fulfilling or rewarding) and a cognitive component (judging that your life is going well for you, by your standards for it). The best way of determining people's happiness levels is to ask them ... However, an individual's report will accurately reflect his perceived happiness only if it is relevant (focused on the prudential dimension of the value of his life), sincere (uninfluenced by the desire to maintain a particular social image), and considered (uncoloured by transitory feelings of elation or depression). The question then is whether happiness, as so measured, is identical to well-being. We have found two reasons for thinking that it is not: a person's self-evaluation may not be informed and may not be autonomous. In either case it is inauthentic, in that it does not accurately reflect the subject's own point of view. Welfare therefore consists in authentic happiness, the happiness of an informed and autonomous subject. This theory of welfare as authentic happiness is clearly subjective.

As with Feldman's hedonism, Sumner here substantially subsumes other theories of wellbeing into his subjectivist theory. The "affective component" of Sumner's theory subsumes hedonism. This is fine, because as discussed, hedonism is inadequate. But it also

\footnotetext{
${ }^{7}$ Another prominent subjectivist theory of wellbeing is that of SW-B. I have declined to discuss this school here for several reasons. The first is that the SW-B literature explicitly distinguishes between short term affect and long-term evaluations of life satisfaction. As such, it is a subjectivist theory that incorporates a hedonistic theory. It already admits the need for integration. Second, there is presently a substantial debate on in the psychology literature between advocates of the SW-B approach and another group who regard themselves as advocating a "eudaimonistic perspective" on wellbeing. This debate is quite lively (see, for example, Kashdan et al 2008; Waterman 2008 and Ryan \& Huta 2009), and the SWB school seems to be rapidly incorporating the eudaimonistic perspective into its own understanding of wellbeing (see, for example, Stone \& Mackie 2013, Graham 2011). As such, it is unclear whether the SW-B perspective can really be considered a freestanding, consistent way of thinking about wellbeing. Finally, the SW-B literature is explicitly atheoretical (Sheldon 2013, Kahneman et al. 1999). The emphasis is on letting the data do the talking and theorising post-hoc. As a consequence, the SW-B literature has a minimalist conceptual understanding of wellbeing and endeavours to avoid theoretical elaboration. As such, it seems wrong to analyse it as a freestanding conception of wellbeing.
} 
seems inaccurate to describe this theory as being really distinct from hedonism. It is hedonism-plus. Sumner concedes this when he says (p. 175):

The wellbeing theory resembles hedonism...in its endorsement of an experience requirement...[but] it also incorporates an information requirement (as part of its condition of authenticity], it is a state-of-the-world theory.

Sumner's theory also subsumes aspects of the objectivist perspective, though he seems not to realize this. This is evident in the requirements of relevance, sincerity and consideration. It is also central to his notion of authenticity, which requires autonomy and being an informed subject. Autonomy is one of the principal objective determinants of wellbeing in SelfDetermination Theory (Deci \& Ryan 2000, Ryan and Deci 2000), and eudaimonic philosophy is fundamentally about discovering and living in accordance with your true self, that is, your daimon (Norton 1976). Sumner's criticism of objectivism is essentially that it gives a poor account of the end state of wellbeing, but he fails to realise that his definition of that state requires a lot of ideas from the objectivist tradition to explain how you get to that end state in practice. This is clear in the passage where he rejects objectivism (p. 175):

[A theory of wellbeing should explain] what it means for a life to be going well not just in itself or from some other standpoint but for its subject. This is, of course, the demand that objective theories of welfare are unable to meet...The authenticity requirement, which is an essential part of the wellbeing theory, guarantees that the operative point of view in a subject's self-assessments of her wellbeing is genuinely hers.

Sumner's critique here is telling for many if not most objectivist accounts, but he overlooks that without self-actualisation, which is one of the principle themes of the majority of objectivist accounts of wellbeing, authenticity is difficult if not impossible to achieve. Selfactualisation requires the harmonisation of discordant aspects of the psyche through integrity, reason and discipline. If self-actualization has not proceeded far, an individual cannot give an honest, authentic account of their own wellbeing.

It is unsurprising in this context that some of the most prominent contemporary objectivist theories are to be found among clinical psychologists (Waterman 2013, Ryan and Deci 2017). Therapists are frequently working with people who lie to themselves and to the therapist, and 
who may be doing well in some ways but not others. A pure subjectivist model is inappropriate in this context because such individuals are not at all of one mind - they are instead compartmentalised, with multiple selves that may be in conflict (Harter 2012). They are an unreliable narrator of their own experience, with an inclination to ignore or repress affective signals that indicate that they are not living authentically. These problems carry over to individuals outside of the psychotherapeutic context as well. ${ }^{8}$

The existence of multiple selves undermines not just life satisfaction theories like Sumner's but also preference-satisfaction theories of wellbeing. People are not a single unified "self" with well-ordered preferences that are more or less "satisfied". Instead, humans are comprised of multiple, often incompatible selves that are sometimes harmonised over the life course through the process of self-actualisation. Through self-actualisation, individuals learn what preferences are "right" for them. Unless self-actualisation is in a very late stage, it is questionable to assume that the preferences people are trying to satisfy will in fact improve their wellbeing if actualised. Preference-satisfaction has, of course, a role to play in selfactualisation and the acquisition of wellbeing more broadly. However, as I will discuss in the final part of this paper, self-actualisation is guided by affective signals and by basic psychological needs for autonomy, competence and relatedness. As such, self-actualisation involves elements from the subjectivist, objectivist and hedonistic traditions. Desire- and preference-satisfaction perspectives struggle to provide a holistic understanding of what wellbeing is and how it is attained without borrowing ideas from other theories of wellbeing.

A final point: Sumner implies that objectivist theories do not describe what wellbeing feels like for its subject. This claim can only be sustained by focusing on classicist works in the objectivist tradition. If you go beyond writings in the Aristotelian tradition, you find many rich descriptions of what eudaimonia feels like. For example, self-determination theory notes that integrated, authentic individuals report their behavior as largely intrinsically motivated and thus easy and fulfilling (Deci \& Ryan 2000). Such individuals also have high levels of positive affect and vitality (see chapter 7 for a longer discussion). Self-discrepancy theory provides empirical evidence that self-concordant individuals report experiencing anxiety and depression infrequently while experiencing positive affective states like exhilaration,

\footnotetext{
${ }^{8}$ Curiously, the need for one's internal psychology to be consistent rather than compartmentalised is a powerful interpretation of what it means to live in accordance with reason, as counselled by Aristotle.
} 
confidence and satisfaction relatively more frequently (Silvia \& Eddington 2012). The eudaimonic individual will also experience flow more frequently (Csikszentmihaly 1992). This is the feeling of being "in the zone" or "lost in the moment".

\section{Objectivist}

Objectivist theories hold that if certain conditions are met, such as virtue, rationality or physical health, an individual is happy regardless of whether they say that they are. What Sumner gets right is that the objectivist theories don't make much sense unless they themselves incorporate subjectivist elements. There are two main things that a good objectivist theory needs to rescue from the subjectivist tradition. The first is that subjectivist ways of understanding the phenomenology of wellbeing, especially the experience of it for the subject, are necessary for a complete theory of wellbeing. The second is a subjectivist theory of value, including ethical values.

In his conception of eudaimonic wellbeing, Aristotle argued that wellbeing can only be achieved by living in accordance with certain values. Specifically, individuals must be reasonable, virtuous and perfect their nature (Annas 1998). These claims have come in for heavy criticism. Kraut (1979) makes a common complaint when he notes that nobody knows what is objectively "good", so virtuousness is an impossible and opaque criterion for wellbeing. Additionally, Haybron (2008), among others, has noted that perfection in the form of fulfilling one's nature is an aesthetic quality rather than a moral one, and certainly has nothing phenomenologically in common with wellbeing. For example, Floyd Mayweather is very close to a perfect boxer, but he is also a wife beater and hardly a moral paragon. The failure of classical eudaimonism to persuade theorists like Sumner comes down substantially to the claim on the part of Aristotle and other classical advocates that the individual must live in accordance with objective normative standards in order to achieve eudaimonia.

Philosophies of wellbeing since the enlightenment that perpetuate the eudaimonistic emphasis on self-actualization but dispense with this notion of objective values, such as existentialism (Sartre \& De Beauvoir 1946) and Norton's neo-Aristotelianism (Norton 1976), are robust to these critiques because they take as one of their foundational assumptions the idea that norms are inherently and inescapably subjective. Indeed, they argue that creating and affirming 
one's subjective values is the only way to achieve eudaimonia. External normative standards are a threat to this process (Nietzsche 1886/1990). In so doing, these theories resist being labelled "objectivist" at all. They draw on ideas from the objectivist tradition, in particular the role of self-actualization in achieving well-being, but define the state of wellbeing and the content of virtue and the good in subjectivist ways.

The emphasis on subjective values in this philosophical literature is also present in psychology schools that advocate a eudaimonistic perspective. Self-determination theory, for example, posits objective psychological needs that define wellbeing but also emphasizes the role of autonomy and being guided by one's intrinsic motivations in the achievement of wellbeing (Deci \& Ryan 2000). It argues that “objective” normative standards could typically only be adhered to by way of extrinsic motivation and self-regulation. Far from producing wellbeing, this would lead to weariness, self-discrepancy and neurosis.

By extension, these modern eudaimonisms argue that the individual must fulfil their unique, individual nature. "Human nature" is here understood not as belonging to the category "human" and perfecting the qualities of that category, such as reason. Instead, human nature is defined by the two existentialist maxims - "man is condemned to be free" and "for man, existence precedes essence" (Sartre and De Beauvoir 1946). What these two maxims amount to is that we must choose who we want to be. Any attempt to escape from this ontological self-responsibility can only be sustained through self-deception, hence why the existentialists referred to such acts as "bad faith". Wellbeing in this eudaimonic tradition arises from actualizing an identity that is self-concordant (Sheldon 2013) — knowing thyself and becoming who you are in Aristotle's language. Aristotle's emphasis on reason and the philosophical life are a product of the fact that his daimon was defined by these things. Other people's daimon's will encourage different callings.

The typical critiques of objectivist approaches to defining wellbeing are telling against classic Aristotelian accounts. However, the more modern eudaimonisms of existentialist philosophy and clinical psychology are robust to these critiques. They are so in large part because they have subsumed ideas from the subjectivist tradition, in particular the idea that "the good" is subjectively determined. Modern eudaimonisms typically establish objective ends that must be met in order for an individual to achieve the experience of wellbeing, but the means by 
which these ends are achieved are deeply subjective. The subjectivist and objectivist understandings of wellbeing are thus integrated in these theories.

The above analysis does not result in an integrated theory of wellbeing. The three traditional philosophical theories of wellbeing canvassed are irreconcilable. However, it does suggest that there is a great deal of overlap between them, especially when you consider how wellbeing is achieved rather than simply what it is. This motivates a deliberate investigation of the similarities between wellbeing theories from a more practical point of view. I undertake such an investigation in the next section. To differentiate the perspective that I use and theory that I develop, I refer to it as the production function theory of wellbeing.

My approach borrows heavily from economics and psychology and from the approach of psychologists working on wellbeing, including those associated with SW-B. In these lines of inquiry, well-being is not defined as the prudential good. Instead, scholars look for correlations and causal relationship between variables linked with wellbeing in folk theories and professional practice, including variables like depression and anxiety that are considered the inverse of wellbeing and variables such as vitality and good moods that are thought to accompany wellbeing. This is a kind of factor analysis approach where scholars study things associated with wellbeing and then try to tease apart what is an ingredient in wellbeing, what is an outcome associated with wellbeing, and what is a part of the causal channels by which wellbeing emerges. Following this approach, I posit a function that defines what factors determine an individual's level of subjective wellbeing. I hypothesise that these are, empirically speaking, objective determinants of wellbeing: individuals without any of these factors will report low SWB; Individuals high in these factors will report high levels of SWB. Furthermore, that individuals high in all of these factors will have greater wellbeing than individuals who are only high in some factors. In the ensuing chapters I present the relevant empirical evidence. In chapter 9, I describe the process by which this wellbeing is achieved. The content of this process will vary greatly from individual to individual, hence why this is a subjectivist theory. It is also gives a critical role to affective signals in guiding the accretion of SWB, so it is partially a hedonistic theory. However, the basic principles of this process, such as self-actualisation through goal attainment, will be the same for everybody. As such, the theory posits that there is a right way to go about pursuing wellbeing and that wrong ways will result in ill-being. As such, I argue that the prudential good must be understood not just 
as an outcome, but also as a process. This is a central claim of eudaimonistic theories of wellbeing, as discussed in detail in chapter 7.

\section{The Production Function Theory of Wellbeing}

The notion of a production function is borrowed from economics. The production function in economics is used to express in mathematical terms what determines the output of a firm. The most common form of the production function is the Cobb-Douglas production function, which is as follows:

$\mathrm{Q}=\mathrm{AK}^{\alpha} \mathrm{L}^{\beta}$

Where:

$\mathrm{Q}=$ Quantity of output

$\mathrm{K}=$ capital (i.e. machinery)

$\mathrm{L}=$ labour

$\mathrm{A}=$ the productivity of labour and capital (i.e. "total factor productivity")

$\alpha$ and $\beta$ are parameters that capture the rate at which the marginal product of capital and labour respectively diminish.

The production function approach to wellbeing would similarly involve specifying some mathematical model of what factors that contribute to increasing someone's SWB, to wit:

$\mathrm{SWB}=\mathrm{f}($.

I begin to populate this function below.

Looking across the full breath of research into SWB, three broad themes emerge. These are whether life is pleasant, whether it is fulfilling, and whether it is valuable. ${ }^{9}$ Hedonic psychologists working on wellbeing, for example, were and still are largely interested in whether life is pleasant. That is their definition of wellbeing (Kahneman et al 1999). Aristotle and the existentialists, in contrast, were mostly concerned with whether life is valuable. They

\footnotetext{
${ }^{9}$ The Japanese concept of Ikigai also incorporates all three of these dimensions of wellbeing (Matthews 2008)
} 
emphasized whether the individual was virtuous and had meaning in their life, among other things. In the fulfilment camp we find, among others, the eudaimonic school of psychology (Deci and Ryan 2000) and Bertrand Russell's (1930) writings in The Conquest of Happiness. These three themes point to a very general specification of the wellbeing production function. In order to better understand these themes and their interaction, I will need to specify them in more detail. I do this by drawing from the literatures most concerned with each of these three themes.

Call the idea that SWB is about a pleasant life the hedonic dimension of SWB. Fixtures of this dimension of SWB include the primary concerns of hedonic psychology: a balance of positive over negative affect, emotional resilience and a positive disposition. There is also an evaluative component to hedonia; call it hedonic life satisfaction. This is an evaluation of the pleasurableness of life as distinct from existential satisfaction, which concerns how valuable and fulfilling life is. Lester Burnham, the protagonist of American Beauty, is high in hedonic life satisfaction but low in existential satisfaction. He is wealthy and comfortable but suffering from ennui and despair.

Hedonia is commonly contrasted with eudaimonia, which concerns elements of SWB that are not emotional or phenomenological in character. I use eudaimonia to describe the cluster of variables that take in whether life is fulfilling. For this cluster I draw on the literature from eudaimonic psychology, specifically the three basic psychological needs emphasized by selfdetermination theory (Deci \& Ryan 2000): autonomy, relatedness and competence. Autonomy concerns how volitional you are in your decisions and the extent to which your behavior is intrinsically motivated. Competence concerns the extent to which you are instrumentally good at the things you care about. And relatedness concerns the quality of your social ties to people and groups you care about.

The school most interested in the normative dimension of SWB-whether life is valuablewere the existentialists, so I will pay homage to them and call this dimension the despair dimension. It is made up of the three principle concerns of the existentialists: nausea, seriousness and anguish. Nausea concerns whether life is meaningful and purposeful, seriousness whether one's ethics are experienced as authoritative and binding, and anguish whether one has a clear sense of self that effectively guides decision making. These themes were prominent in early continental psychology (Frankl 1946) and continental philosophy, 
and also appear in the writings of positive psychologists (Baumeister 1992, Ryff 1989, Seligman 2011).

Putting these factors together, we arrived at a specification of the wellbeing production function that is sufficient for our needs for the time being:

$\mathrm{H}=\mathrm{f}($ hedonia, eudaimonia, despair)

Where

Hedonia $=\mathrm{f}($ affect, satisfaction, emotional wellbeing $)$

Eudaimonia $=\mathrm{f}($ autonomy, competence, relatedness $)$

Despair $=\mathrm{f}($ anguish, nausea, , seriousness $)$

The above specification of the wellbeing production function is obviously intended as a sketch. Other authors have advocated for different demarcations to the one I am proposing. Baumeister (1992), for example, places most of what I have under the headings of eudaimonia and despair under the single heading of "meaning". I defend my specification over the course of chapters $6-9$. Before that, however, it is important that I complete this sketch. Everything I discuss here will be repeated in greater detail in later chapters.

In the next section I analyse the role self-actualisation plays in satisfying all three of hedonia, eudaimonia and despair. This is not to suggest that self-actualisation is some sort of metastrategy for SWB that subsumes all others. There is a lot of value in studying strategies appropriate to the acquisition of any one of the dimensions of wellbeing, such as Sheldon et al's (2013) hedonic adaptation prevention model. I discuss these in some detail in chapter 6 . Rather, it is to show how important self-actualisation is to the praxis of SWB, the extent to which the three dimensions of SWB are interrelated and interdependent, and the usefulness of adopting a perspective that keeps a firm eye on the praxis of SWB.

\section{Self-actualisation and the practice of wellbeing}

To talk about the practice of SWB we need to ask the question: what makes you well? Some things come to mind. These include doing the things you love, being the kind of person that 
you love, ${ }^{10}$ and making the world a better place. In this section, I hope to show how selfactualisation can bring about such things and ultimately lead to SWB. The account of selfactualisation given below is a sketch of the coalescence of being.

Let's begin by describing the core of the self-actualisation process. I borrow this from selfdiscrepancy theory (Higgins 1987): you harmonize your "actual self" with your "ideal self" and "ought self". Your actual self is who you are at the moment, including the things that you value and the things you find intrinsically motivating and pleasurable. Your ideal self is who you would like to be, and your ought self is who you have a responsibility to be.

Your ideal self can be thought of as a constellation of values and their associated behaviours with which you identify; the-ought self is similar, but the values therein are specifically ethical values. "Identification" is the first stage of intrinsic motivation in self-determination theory (Deci \& Ryan 2000). Unlike duress or introjection, which are extrinsic kinds of motivation, identified behaviours are somewhat self-determined rather than entirely selfregulated. This spectrum of motivation can be understood by thinking about swimming. Many people value health and pursue exercises, such as swimming, as a means of maintaining their health. Identifying with health provides some motivation, but swimming for some people, because they are unskilled and unfit, is arduous and requires willpower. It is thus an extrinsically motivated activity in that one needs to regulate aspects of one's psyche that want to exit the pool in order to proceed. As such identified behaviours become assimilated into the self they start to connect up to other values. For example, swimming for health might inadvertently make one more aesthetically pleasing to oneself. One might also join a triathlon club to help with swimming, thereby associating swimming with one's other values for community groups and athletic competitions. In this way, swimming becomes integrated, which is the final stage of internalisation before intrinsic motivation.

The basic idea in self-discrepancy theory is that you should identify who you want to be with the help of your innate motivational compass and then try to become that person (this is reminiscent of the Hellenic maxim, repeated by Nietzsche and Norton: "become who you are"). This includes moving away from the "feared self" - the individual you especially do not want to be. The process by which you bring your actual self into accord with your ideal self is guided by affective signals that accompany what the existentialists called the

\footnotetext{
${ }^{10}$ Adam Smith (1759) put this eloquently when he said: "man naturally desires, not only to be loved, but to be lovely; or to be that thing which is the natural and proper object of love" (Smith 1759).
} 
"disclosure of being". This is where who you are in reality is revealed to you in your actions or the observations of others. For example, if you desire to be fit and consequently swim $1 \mathrm{~km}$, this indicates to you that you are somewhat fit. You will experience positive affect as a result because you are revealed by your actions to be closer to who you ideally want to be. As you swim further, faster and more frequently, you will more thoroughly confirm this observation that you are fit, and this will make you happy and enhance your SWB.

If you do not complete the $1 \mathrm{~km}$ swim, this will result in negative affect (Silvia \& Eddington 2012). There are a few ways to interpret this negative affective signal. If you are depressed because you didn't complete your objective, you need to redouble your efforts. However, if you are instead disenchanted by swimming, it might simply be that swimming doesn't suit you - it is not in accordance with your true self (Sheldon 2002). Your self (or daimon) is communicated to you by the degree of motivation you experience for certain values and activities and the corresponding affective signals you receive when undertaking those activities and affirming those values. Now if fitness is core to your personality but swimming is not a self-concordant way to achieve fitness, you just need to find a more suitable sport. However, it could be that fitness itself is the unsuitable value, in which case you may need to abandon it. In order to correctly interpret your affective signals, you will need to do some introspection and rationally engage with your feelings. ${ }^{11}$ This introspection is a major theme in narrative therapy (McAdams and Janis 2004) and some cognitive behavioral therapy exercises (it is also fundamental to the other great Hellenic maxim: "know thyself").

This process of harmonisation with the actual self through iterative engagement with the world and introspection is similar for ethical values and the ought self. Perhaps you identify with recycling because you think that the world has finite resources and we should protect them for future generations. Recycling takes a bit of effort initially as you learn to sort your rubbish and motivate yourself to carry your loads to the neighbourhood recycling bins, but gradually it comes to be easy. You get a warm feeling from recycling that makes the effort worthwhile, and you integrate the trip to the bins into your weekly grocery run. You also start to organise with local groups to make recycling easier and the community more aware of its benefits. The council follows through on your suggestions, and the world is now a little bit more the way you'd like it to be.

\footnotetext{
${ }^{11}$ See Tiberius (2008) for a longer discussion of what this introspection might look like.
} 
Now imagine that one day you are watching a documentary on television that shows how glass recycling actually takes more energy that it saves, and that the other resources involved, namely sand, are abundant (Munger 2013). You find it very persuasive. At this point, you might need to adjust your recycling values to not include glass, and this might bring you into conflict with some of your old comrades in the neighbourhood recycling group. Here, not only will you need to introspect about your own values, but you will also need to develop arguments, especially rational arguments, with which to convince your colleagues of your own views. This will force you to systematise your thinking about your own values. ${ }^{12}$

The above is a simple explanation of self-actualisation using crude examples, but it already reveals how self-actualisation brings about SWB. First, guided by your affective signals, you mostly do things that you find intrinsically enjoyable, and gradually eliminate extrinsically motivated activities and values from your life. This achieves a balance of positive over negative affect as your behaviour gradually comports towards positive affect and away from negative affect. It also nurtures your sense of autonomy. As you become good at these pursuits following sustained engagement in them, you feel more and more competent at things that you care about. This is exhilarating and provides a source of self-esteem that makes you resilient to depression and anxiety, thereby fostering your emotional wellbeing.

You also pursue ethical values that you find intrinsically attractive. This pursuit of values is likely to bring you meaning and give you purpose because you are making the world a better place. This overcomes nausea. You pursue these values because you want to be the kind of person who lives in accordance with them - that person is your ought and ideal self, and achieving that identity gives you positive emotions. Your pursuit requires integrity. This necessity for integrity overcomes the problem of seriousness because your values are serious to you and you are capable, indeed incentivised, to impose them upon yourself. ${ }^{13}$ As you pursue your values and try to make the world a better place you necessarily come across and socialise with other people who share your values, and organise with them to oppose groups with antithetical values. This nurtures your need for relatedness. ${ }^{14}$ This process of self-

\footnotetext{
12 This provides some more texture to what Aristotle might have meant by "living in accordance with reason".

13 This links self-interest and ethical behaviour, the holy grail of much ethical philosophy. It also addresses one of the major sticking points in existentialist philosophy, namely how to overcome the seeming capriciousness of subjectively chosen values.

${ }^{14}$ It is worth noting that relatedness and community are hereby brought about in a way that maintains individual autonomy. Many scholars and commentators urging greater emphasis on the role of community in wellbeing, notably Haybron (2008, chapter 12) overlook this and thereby condemn misfits and deviants to a life of oppression on the part of groups they don't want to belong to.
} 
actualisation also sees you come to better understand yourself gradually because of the disclosures of being that you witness, the introspection and rational analysis of yourself that you undertake, and the emotional signals that you get from yourself. The end result is that you come to know and be who you are, which annuls anguish. All the different dimensions of wellbeing have been accounted for: hedonia, eudaimonia and despair.

\section{Implications of the production function perspective}

Several noteworthy points emerge from the production function perspective. First, as discussed above, self-actualisation promotes all the different dimensions of wellbeing. It is clearly important, and its particularities perhaps deserve more theoretical and empirical attention from both philosophers and psychologists.

Second, taking a practical perspective on wellbeing reveals how the different dimensions of wellbeing are interrelated. For example, self-actualisation resolves despair through the pursuit of values and goals with integrity. At first, the despair dimension might not seem to have much to do with emotions, certainly not with positive emotions like exhilaration. Yet the selfactualisation process that dampens despair is guided by affective signals that include things like exhilaration, enchantment and joy. Thus the despair and hedonia dimensions are interrelated. The same can be said for the basic psychological needs that make up the eudaimonia dimension. Pursuing activities that are intrinsically motivated and bring us into contact with groups that share our values helps us to feel autonomous, related to people we care about and, ultimately, competent. Yet we rely on affective signals to help us sense whether we are undertaking an intrinsically motivated activity or instead a self-regulated one, and we require notions from the despair dimension to understand our values and thereby help us to identify comrades. The dimensions are again interrelated. It might seem like the affective dimension is the critical one here as it is the guide to the deeper dimensions, but the relationship goes both ways. It is hard to understand why we feel good or bad about particular behaviours, values and groups without understanding the eudaimonic and despair dimensions. As such, a narrow focus on the hedonic dimension of wellbeing would miss crucial issues related to the causation of happiness and wellbeing.

Third, self-actualisation is important to the practice of wellbeing regardless of what traditional theory of wellbeing is used and there are aspects of all three theories present in the 
production function perspective. You will need to feel pleasure in order to be happy in the long run because without pleasure your self-actualisation will not progress. This is the hedonistic part of the theory. You will also need to satisfy your preferences-these can be understood as the goals that are inherent to the ideal and ought selves. These preferences will initially be unclear to you and may even be wrong. Hence you will be inauthentic when you express them. However, as self-actualisation proceeds and you come to know thyself and become who you are, you will discard self-discordant preferences and refine self-concordant ones through introspection. You will consequently end up satisfied with your life in a considered, authentic and autonomous way, as in Sumner's subjectivist theory of wellbeing. Finally, you will need to live in accordance with reason because rational introspection is required to understand your affective signals. Furthermore, rational reflection is necessary to resolve compartmentalisation and inconsistencies in your identity. You will also be a virtuous individual because without commitment to your ought self you will not achieve seriousness, meaning and relatedness (though of course what "virtue" is for you will ultimately be defined by you). As such, you will satisfy themes from the objectivist tradition.

Finally, the coalescence of being account of self-actualisation reveals that defining wellbeing as the prudential good is problematic. Coalescence shows that individuals only come to know what is good by pursuing their SWB. Defining wellbeing as the prudential good thus invites confusion. It puts the cart of goodness before the horse of wellbeing. Furthermore, the coalescence account argues while the individual is ultimately the arbiter of what is "goodfor" themselves, they only discover this good by following affective signals (hedonistic "goods"), by behaving rationally and with integrity (Aristotelian "goods"), and by pursuing the nourishment of their basic psychological needs (eudaimonic "goods"). As such, they only arrive at their subjective understanding of what is "good-for" themselves by following "goods" from the hedonistic and objectivist traditions.

\section{The way forward}

The first half of this chapter pried open some space in the analytical philosophy cosmos for a new theory of subjective wellbeing derived from scientific approaches to wellbeing. The second half of this chapter was then a sketch of both the production function theory of wellbeing - a model of what wellbeing means as an outcome variable — and the coalescence of being - a model of how self-actualisation achieves that outcome. In the next chapter I 
present the production function in full, including a more thorough mathematical specification. There are then five chapters justifying, explaining and analysing each of its component parts. The last of these, chapter 9 , goes into great detail on the nature of the coalescence of being. 


\section{Chapter 4}

\section{The Wellbeing Production Function}

My soul is like a hidden orchestra; I do not know which instruments grind and play away inside me, strings and harps, timbales and drums. I can only recognise myself as symphony

- Fernando Pessoa, The Book of Disquiet

\section{Introduction}

This chapter outlines the contours of the wellbeing production function and provides a formal, mathematical specification. Chapters 5 through 8 then justify and explain each of its constitutive elements in greater detail. I discuss in turn the agency constraint, hedonia, eudaimonia and despair drawing extensively on the relevant literatures in psychology, philosophy and occasionally economics.

Before continuing, I'd like to address a concern that some readers might have at this stage that this project of developing a holistic model of wellbeing is ambitious; indeed, overly ambitious given the state of our existing empirical understanding of wellbeing. On the one hand, I am sympathetic to this perspective. When theory gets too far ahead of empirical confirmation it opens the possibility of ultimately wasted effort when a large body of theoretical work is later refuted by empirical findings. On the other hand, I see a lot of value is outlining the entirety of wellbeing for two reasons.

First, there is plenty of potential for wasted empirical effort if incremental gains in some relatively narrow area of wellbeing scholarship are disconfirmed by or otherwise incompatible with findings in some other relatively narrow area of scholarship. This is a very real possibility in wellbeing scholarship because its wide interdisciplinary scope means that there are several streams of research at present that don't talk to each other much, if at all. If 
they do communicate more in the future, researchers in each stream might discover that their work runs counter to a body of knowledge in some other silo. An example is Annas' (2004) paper on classical philosophical perspectives on happiness. This piece was prompted by an email notifying her that she had been included in the World Happiness Database, which at the time was a repository of research in SW-B. Upon perusing that resource she felt that Aristotelian perspectives were unrepresented in it. This was a problem these perspectives called into question research in SW-B yet were unaddressed by SW-B scholars.

Second, there are numerous examples in other areas of social science research where the statement of a "general theory" aided the investigation of specific branches of that general theory and helped them to feed their findings back into the general theory. An illustrative case is inquiry into the nature of the education production function. Several decades ago, the Coleman Report (1966) proposed that education was a function of student characteristics like general intelligence and effort, school characteristics like teacher quality and class sizes, household characteristics like income and parental conflict, and neighbourhood characteristics like socio-economic status and culture. At the time, the magnitude of the effects of each of these domains was ambiguous and the exact details of the functional form of the education production function were unclear. Nonetheless, the statement of the entirety of the function clarified the research landscape and illuminated, among other things, what interrelationships might exist between relevant variables. This clarification at the aggregate or meta-level was helpful to more precise empirical scholarship of individual variables like the impact of school infrastructure on education outcomes (Hanushek 1986).

I am attempting something similar here with the wellbeing production function. I lay out a general theory in the hopes that it will guide more specialised inquiry and feed the results of those inquiries back into the general theory. The chapter begins with the first order structural equation of the wellbeing production function. It then discusses the second order structural equations for each variable in the first-order structural equation. With the key variables thus specified, the chapter turns to discuss important parameters, in particular emotional wellbeing, nihilism, personality and reference points. Once these are articulated, the wellbeing production function is specified formally. An estimation strategy is discussed in chapter 10 . 


\section{The wellbeing production function}

Wellbeing is a function of three variables: hedonia, eudaimonia and despair. These correspond to whether life is pleasant, whether it is fulfilling and whether it is valuable, respectively. Hedonia is in turn a function of positive affect, negative affect, and hedonic life satisfaction. In the subjective well-being literature, positive and negative affect (i.e. positive and negative emotional states) are widely regarded as being two separate spectrums rather than a single bipolar "mood" variable (Tugade and Fredrickson 2004, Kuppens et al 2008). For example, you can be simultaneously in a state of high positive affect, such as exhilaration while watching the latest Hollywood action movie, and negative affect, such as stress at your impending deadlines. As such, they are entered into the model as separate terms. Hedonic life satisfaction refers to how pleasant you evaluate your life as being.

Note that Hedonic life satisfaction is distinct from existential satisfaction, which is captured by the eudaimonia and despair terms. You can evaluate your life as pleasant while still being unsatisfied with it and/or generally unwell. For example, your typical OECD university student of middle class background afflicted with nihilism might say that they "can't complain" about their life, because they are healthy, safe, comfortable and entertained. They are high in hedonic life satisfaction. However, they may also feel lonely, empty, purposeless, dislocated and unmotivated. They are low in existential satisfaction. I am personally and intimately familiar with this state. As far as better understanding the structure of responses to life satisfaction scale questions is concerned, the distinction between hedonic and existential life satisfaction seems a valuable one.

Eudaimonia in this model is a function of the three basic psychological needs emphasised by self-determination theory (SDT): autonomy, competence and relatedness. Autonomy refers to how volitional one feels in one's behaviour. Competence to how capable one is convinced one is at things one cares about. And relatedness refers to the depth of one's social ties to people one cares about. I say "in this model" because in the philosophical literature wherein the concept of eudaimonia was born it is typically associated with normative dimensions of wellbeing, in particular the notion of virtue and living in accordance with it. The three basic psychological needs of SDT are only tangentially associated with virtue. I nonetheless use eudaimonia to describe this cluster of variables because Richard Ryan, Edward Deci and other theorists of psychological wellbeing self-identified as advocating for a eudaimonic perspective on wellbeing in distinction to a hedonic conception when they first started 
engaging with the subjective wellbeing literature (Ryan et al 2008, Waterman 2008, Kashdan et al 2008). Another reason is that the literature on despair is much more explicitly focused on normative issues than the eudaimonic psychology literature, and the despair literature is in some ways the evolution of the eudaimonic literature in philosophy.

Despair is a function of nausea, seriousness and anguish. All of these terms are drawn from existentialist philosophy, and their meanings are not strictly in accordance with common usage. Despair, for example, is not the opposite of hope and optimism. Those are affective states. Instead, despair is best understood as analogous to what Frankl (1946/2008) called existential vacuum.

Nausea refers to the sense that the world and the individual in it is without purpose. Furthermore, that any meaning we bring to the universe through endeavour and creation ultimately crumbles to dust from entropy, and so meaning can only be sustained by our drives, attention and love (Reginster 2009). There is nothing transcendental in the universe, no grand cosmic plan, no "meaning of life".

Seriousness is the sense that the world is devoid of moral order. In Nietzsche's words, "there are no moral facts". As research in the evolutionary psychology of moral cognition has shown, morality evolved as a cognitive module that helps humans cooperate in groups (Haidt 2012, Greene 2014). Ethical value is not an essential property of things, but rather a quality that humans imbue things with that is then sustained by inter-subjective belief. Moral rules are thus "made up" and therefore arbitrary. The world is a morally relativistic place. In this context, morality loses its authority, its binding power. As Dostoevsky noted so eloquently, "all things are permitted". Ethics are no longer serious. This is a distressing realisation for humans because we are wired to operate within communities sustained by normative architectures that we regard as serious. We are also wired to ostracise and potentially even assault individuals who transgress these serious ethical rules. If the rules are made up and trivial, then we have no transcendental grounds for this exclusion and oppression. We are also without any effective normative anchor in the world. Despair ensues.

Anguish refers to the absence of a palpable identity that we can use to make decisions (Sartre 1943/2005). Humans are always in a state of "becoming"-we are defined anew by decisions we make moment to moment. As such, we are always ontologically free to break commitments we have made to ourselves in the past or even completely rewrite our identities. This is a profound privilege because we are responsible for the creation of our essence, but it 
is also a difficult burden because we must always justify our conduct to ourselves and are perpetually cut off from a definitive understanding of who we are. The normative element of anguish is that we are defined substantially by our values - it is these that we affirm and manifest in our choices. Existential vacuum is a state where nausea and the problem of seriousness combine such that we do not easily experience values as deterministic, and consequently our choices become difficult and our affirmation of them feels empty. We are more easily "anguished", literally, because the question "what should I do?" finds no definitive response.

At this juncture, a flowchart might help clarify what SWB is a function of. I will present a more formal specification after discussing parameters in the next section.

\section{SUBJECTIVE WELLBEING}

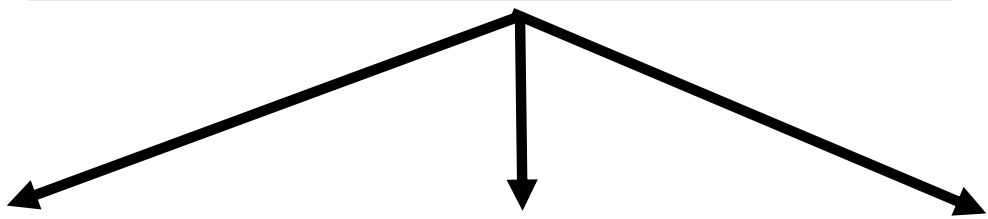

\section{Hedonia}

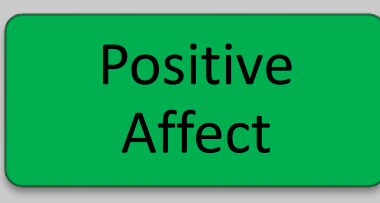

Negative Affect

Hedonic Satisfaction
Eudaimonia

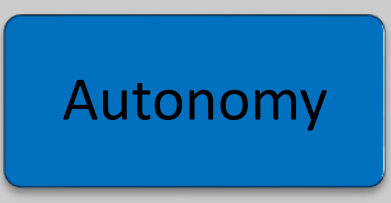

Competence

Relatedness
Despair

Nausea

(Meaning)

Seriousness

(Values)

Anguish (Identity) 


\section{Model parameters}

There are two important parameters that affect one of the first-order variables of the wellbeing production function. These are emotional wellbeing, which parameterises hedonia, and nihilism, which parameterises eudaimonia. There are two additional parameters that affect the entire production function. These are personality and relative status effects. I will first discuss parameters that reflect the interrelationships between the three dimensions of wellbeing — hedonia, eudaimonia and despair — before turning to personality and reference group effects.

\section{Emotional Wellbeing}

Hedonia is parameterised by emotional wellbeing, which is a function of eudaimonia, despair, attention and flow. Emotional wellbeing refers in this case not to your present emotional state, which is captured by the affect variables, but to your emotional disposition. This was discussed in the previous chapter with reference to Haybron's (2008) analysis of hedonism. Emotional wellbeing captures your tendency towards depression, for example, the resilience of your good moods to negative shocks, optimism versus pessimism, hope and other factors that affect the dynamics of your mood - its tendencies in terms of stability and change.

Emotional wellbeing has two broad parametric effects. The first is that if your emotional wellbeing is low, you will struggle to achieve a high degree of positive affect and a low degree of negative affect. It is hard to put a depressed person into a good mood, even with things that have in the past elicited a strong positive affective response from them. Or consider another example: someone who has low self-esteem (an affective state) because they feel incompetent (an aspect of eudaimonia) will be hard to cure of their low esteem by way of mood interventions. The source or cause of the low self-esteem, namely that person's belief that they are incompetent, will need to be addressed first. The second parametric effect of emotional wellbeing is that it affects the speed of adaptation of mood to negative and positive shocks. The lower someone's emotional wellbeing, the faster they will return to a low baseline following positive shocks. The effect of negative shocks will also be more persistent. Consider someone who is anxious because they have recently contradicted, in a grievous way, the morality they are trying to affirm as part of their ought-self. Taking this person out 
dancing might distract them for a few hours, but the anxiety will rapidly return until the object of their guilt is addressed. Individuals low in eudaimonia and high in despair will have transient and fragile good moods.

Emotional wellbeing is a function of eudaimonia and despair because the affective signals such people receive day to day will frequently draw their attention to their unmet psychological needs or the urgency of finding meaning in life. They will consequently have little cognitive ability to focus on potential sources of good mood and the things in their life that they find pleasing.

The other principle component of emotional wellbeing is "attention". Attention in this context is a catch-all for various cognitive-behavioural strategies for managing mood, which include the very straightforward idea that focusing your attention on the positive things in your life will put you in a better mood than focusing on the negative things (Dolan 2014). This is the literal meaning of "attention" and forms a core part of the hedonic adaptation prevention model (Armenta et al 2014). Other techniques in this suite come overwhelmingly from research in hedonic psychology and include savouring, gratitude, humility and other positive activity interventions, as well as the general idea of maintaining a positive and optimistic attitude, which helps to avoid learned helplessness (Seligman 1992). I will discuss these techniques in greater detail in chapter 6 in the context of hedonia.

It is debatable whether "flow" should be included in emotional wellbeing. Flow is a state where one is "in the zone" and is typically associated with high positive affect and existential satisfaction (Csikszentmihaly 1992). It requires the individual to engage in tasks that are intrinsically motivated, have immediate, high quality feedback, and in which the individual has a high degree of skill but is nonetheless challenged. In this sense it is a particular kind of attention. However, in order to achieve flow consistently and in a wide variety of life domains, individuals need to progress quite far along the coalescence of being. As such, I am unsure whether to think of flow as a dimension of emotional wellbeing or as a framework through which to understand coalescence. For the time being, I include it as part of emotional wellbeing. I will discuss flow in greater detail in later chapters. 


\section{Nihilism}

Eudaimonia is parameterised by nihilism. This is a cognitive state wherein the individual finds it difficult to make the psychic movement towards transcendence, which is the philosophical term for finding meaning and identity and experiencing one's values as serious (De Beauvoir 1949/2011). The cynical worldview at the heart of nihilism, which perceives the world and life as objectively pointless, is factual accurate. As such, nihilism is hard to escape once you have succumbed to it because it is not inherently erroneous. Transcending nihilism requires the individual to bring meaning and value into the world and thereby define themselves as an identity. This requires some conviction that the things the individual does "matter" (in the sense that they have a point) simply because the individual "cares" about them (Heidegger 1927/1962). But this care is always under assault from the nihilistic instinct that things aren't worth caring about because everything turns to dust in the end. The extent to which these instincts dominate conscious attention is what is captured by the nihilism parameter.

It is worth nothing that most people do not suffer from nihilism. They are held up out of it by culture and other mechanisms by which humans are socialised into value systems and identities that they experience palpably enough to stave off nihilism. The reason why nihilism welled up in Europe in the 19th century, ultimately culminating in totalitarianism, is that this era saw massive declines in the domination of religious values and belief following the enlightenment (Nietzsche 1891/1978), the emergence of atomising metropolises that broke down the systems of small community life (Simmel 1903/1950), and the transition from citystates to nation-states. All of this amounted to a tremendous shift in group allegiance at the level of individual identity. The traditional sources of socialisation into palpable normative systems and communities that nourished competence and relatedness (though not autonomy, which was arguably a major driver of the enlightenment transformation) were weakened in the space of a mere century or so. Totalitarianism, whether fascism or communism, with its clearly defined normative codes, penchant for grand demonstrations of social solidarity in parades and the like, and organisation of individual life through work groups, party clubs and central planning, effectively filled this vacuum (Jung 1928/1933, Fromm 1941/1994).

A similar phenomenon is arguably taking place today in the English speaking countries, especially America. The end of the cold war, the decline of smaller towns as a result of globalisation, the destabilisation of religious belief by science and the divergent values of 
rural and urban America are all fomenting an existential crisis. People are desperate for identity, especially group identity (hence the rise of identity politics), normative seriousness (hence the political animosity) and purpose (Sides et al 2006). Nihilism could be easily and fairly dismissed as a niche issue or even a mental health disorders by American psychologists in the past, but will become a bigger issue in coming decades.

Nihilism undermines the acquisition of each of the three components of eudaimonia. Most obviously, it undermines the veracity and authority of the normative systems that underpin groups. The ways in which we are "related" to each other come to feel arbitrary and fictitious. A nihilistic individual can feel competent, but the power of this self-belief to give rise to feelings of wellbeing is undermined by the nagging question "what is the point in being competent at this?" The achievement of competence requires persistent effort in the same direction. Such motivation is hard to sustain in the face of nihilism. The relationship between nihilism and autonomy is less straightforward. Nihilism and despair arise out of our ontological freedom (Sartre and De Beauvoir 1946). Consciousness gives us a sense that we have free will. In the moment of decision we perceive ourselves as completely at liberty from any kind of determinism. Not even a gun to your head or the threat of eternal hellfire takes away our capacity to choose the bullet or damnation. Sometimes we don't know what to do with this freedom - we don't know what the correct choice is in a situation. Yet we also know that this decision will define who we are into the future. This is what the existentialists called "anguish". Anguish makes our autonomy salient, but it also cuts us off from identity and confronts us with the need to effectively justify our decisions. Anguish leads us to ask the question "who is it that is making this decision? Who am I? What defines me?" A failure to answer these questions definitely undermines feelings of autonomy.

One might ask whether it would be more straightforward to parameterise eudaimonia with despair rather than introducing the additional concept of nihilism. Yet nihilism and despair are distinct. You can be high in despair and still relatively high in nihilism - this is how I would describe myself. Much like emotional wellbeing's effect on hedonia, an individual high in nihilism is fragile with regards to their sense of meaning, seriousness and identity and prone to bouts of existential anxiety. It is a psychological tendency. On the other hand, you can also be high in despair and low in nihilism. This would describe most people in primitive tribes or anyone else who has been raised in a thick normative system that they slot comfortably into, like many people in contemporary Japan or Amish communities. The normative community provides seriousness, meaning and identity in the form of designated 
social roles (notably gender roles), dampening despair. Slotting into this normative community effectively grants a sense of competence and nourishes feelings of relatedness. Eudaimonia is assured. Such an individual has neither nihilism nor despair. Finally, you can be high in nihilism and low in despair. The "suicidal nihilist" of popular culture is a clear example, but a less dramatic one is the bourgeois university student afflicted by existential malaise mentioned earlier. This individual's nihilism inclines them to see the world as pointless despite all the many exciting and wonderful things happening in it, and to see themselves as undefined despite belonging to a range of groups, possessing hobbies and affirming values.

\section{Personality}

There is extensive empirical evidence to suggest that personality has a strong effect on how well people believe themselves to be (DeNeve and Cooper 1998, Steel et al 2008). Individuals high in openness and extraversion have been repeatedly found to have more positive moods on average, while individuals high in neuroticism and introversion have more negative moods (Bouchard et al 1990, Steel et al 2008, Lucas and Diener 2009). Personality has also been found to play a mediating role in adaptation to changing circumstances. For example, individuals high in conscientiousness struggle to adapt to unemployment (Boyce et al 2016). By extension, changes in personality over time can predict changes in SWB. For example, individuals who become less conscientious while unemployed adapt faster to unemployment (Boyce et al 2013, 2017a). Recent research exploring the link between hope and optimism has found strong impacts on emotional wellbeing, SW-B and long-run life outcomes, like sustained effort to exit poverty (Bailey et al 2007, Hutz et al 2014, Graham 2017). The complex interplay between personality, personality change and SWB is only now becoming a topic of extended, in-depth inquiry but if recent publication rates are any indication this is a rich vein of research (Boyce et al 2017b).

\section{Relative Status and Reference Group Effects}

A final parameter to consider is how to model relative status effects. There is now an abundance of empirical evidence that suggests people care almost as much about their relative income as they do about their absolute income (Knight and Gunatilaka 2014, Fritjers 
and Mujcic 2012, Clark et al 2008b). There is also a long history of inquiry into more generalised "status anxiety" in sociology and anthropology (Veblen 1899, Bourdieu 1979). People care about not just how good their own life is, but how good their life is relative to the lives of others.

Hedonic psychologists and happiness economics have demonstrated extensively the effect of relative status on affect and hedonic life satisfaction. The extent to which people evaluate their lives as comfortable is determined in part, for most people at least, by their neighbours (Brown et al 2008, Bhuiyan 2018, Clark and Senik 2014b). This is one origin of the ubiquitous phenomenon of "keeping up with the Joneses". A parallel phenomenon is the ebullient mood of the poor in the developing world, which has been recognised in the subjective well-being literature under the rubric of the "happy peasant" (Graham 2012). The poor compare themselves to other poor people in many cases, and thus their relative poverty does not burden them as much as it might affect a worker in a rich neighbourhood.

It is less clear how relative status affects eudaimonia and despair. Nihilism is largely a first world problem. It first reared its head in Europe at the height of that region's power in the period from 1850-1950, and it is today emerging in America on the heels of America winning the Cold War and becoming the first global hegemony. One could further argue that it is overwhelmingly the concern of the cultural elite. In Europe, it was most prominent among intellectuals and artists and in America today its epicentre is the university campus and apex media establishments like The New Yorker and The Atlantic. These anecdotes would suggest that despair is mostly a problem for those on top in a society.

However, many of the tropes of despair are today most evident in the American rust belt states, and similar phenomenon were present among Europe's working class during its existential crisis. Europe's totalitarian regimes tapped into a strong and very broad-based desire for normative order and relatedness with their nationalist, historicist and racist narratives. In the USSR and Germany, there was also a strong appeal to competence and autonomy. In Germany the narrative was about restoring German pride and place after the humiliation of the Versailles treaty. In Russia it was about self-determination for the proletariat. Moreover, Europe's totalitarian regimes were distinctly anti-elite and antiintellectual. The same can be said of the contemporary backlash against urban liberals and foreign workers among despairing rural Americans, who decry the elites' intellectual assault on what they consider common sense in the form of say, gender-segregated bathrooms, gun 
rights and abortion. These tropes would suggest that despair is just as if not more liable to emerge among the downtrodden.

Looking directly at the individual components of despair, there is reason to believe that relative status could affect them. Competence, in particular, is often measured relative to other people and verified by authoritative peers. Art, for example, is notoriously prone to trends and cliques, and one's reputation as an artist depends significantly on the opinion of others rather than one's technical brilliance. Even in cases that we could consider clearer cut, like mathematics, there are complicating cases. John Nash, the progenitor of game theory, suffered a complete nervous breakdown arguably because he believed himself to be a genius doing magnificent work but he was unable to gain recognition for it. Even in cases where one can get absolute feedback about one's competence, whether one is "good" at that thing often depends on relative considerations. In weightlifting for example, one can transcend one's personal best, but whether one is an outstanding weightlifter nonetheless depends on whether one can lift more weight than anyone else. These relative effects on competence flow on to anguish through the channel of identity. We often define ourselves in distinction to others and in terms of our achievements, which depend at times on being better than others.

Autonomy seems largely independent of relative status effects, but nausea, seriousness and relatedness could all be affected. For most people, one's sense of meaning and seriousness is dictated in part by the extent to which the group one belongs to flourishes. The despair and desperation of rust belt voters in America's 2016 election is driven in part by a perception that their communities and their way of live are in decline relative to others, notably those of black and urban communities and emerging economies to which industrial jobs have been offshored (Graham 2017).

The effect of relative status on relatedness can be understood through the lens of "popularity contests". One might have a good level of relatedness in absolute terms but be dissatisfied with it because in relative terms one is unpopular or not the queen bee. To lean on popular culture tropes, one might be an accepted member of the cheerleading squad, but because one is not the head cheerleader and dating one of the star players of the football team, one feels dissatisfied with one's level of relatedness. These sorts of sentiments are certainly toxic and undermine feelings of relatedness. They smack of extrinsic aspirations - goals that are pursued for reasons contingent to the goals themselves, like fame, money, or status (Kasser and Ryan 1993, 1996). As discussed at length in chapter 8, the pursuit of extrinsic aspirations 
is negatively associated with wellbeing and even their attainment is at best only weakly positively associated with wellbeing. So it is healthy to not care about relative status and people should work on caring less about it, but people do care, and this affects (negatively) their level of relatedness.

In summary, it seems reasonable to conjecture that relative status exerts parametric effects on every dimension of the wellbeing function. In the next section I discuss the agency constraint. Individuals attempt to maximise their wellbeing function subject to a constraint imposed by their agency and information they have about what would actually make them happy. I do not model the information issue, but I do discuss it in chapter 5 and the coalescence of being, discussed in chapter 9, is a model of how it is alleviated.

\section{The Agency Constraint}

In the vast majority of subjective wellbeing research, the conventional objectives of economic development are taken to exert a causal influence on wellbeing (Clark et al 2018). I take a different view. I argue herein that income, health, education, political enfranchisement and environmental quality are a constraint on an individual's ability to achieve wellbeing, rather than a direct source of that wellbeing. I specify this as an "agency" constraint. I use the term agency rather than freedom-Sen's (1999) preferred term-because freedom is only one aspect of agency. Being healthier while imprisoned for example, does not improve one's freedom, but it does improve one's agency. One can more easily hold one's own in the yard, for example, or keep oneself entertained with exercise.

The reason why these variables should be thought of as a constraint rather than part of the wellbeing objective function is that they merely help you to achieve the things that give you SWB; they do not themselves provide SWB (though they do provide welfare). This idea is embedded in the standard consumer problem of neoclassical microeconomics. Income in this literature is not a source of utility but rather something that you need in order to purchase goods that you prefer. It is these goods that are the actual cause of your utility. The same is true of the other "capabilities". As your health improves, for example, your capabilities expand; perhaps you are now able to run a marathon. However, unless you actually want to run a marathon, this has little effect on your SWB. And so too for the other variables, even political enfranchisement: if the degree of enfranchisement you experience in your society is 
sufficient for you to genuinely undertake all the activities you desire, then the fact that your enfranchisement is not as good as possible does not have a significant impact on your SWB.

The most important ramification of taking agency as a constraint rather than a source of SWB is that it formalises this idea that people need to act on their agency to achieve SWB-agency is the means, SWB is the ends. Further increases in agency beyond a certain minimum don't have appreciable effects on SWB because they don't actually help the person to selfactualise. They already have everything that they need for that - except information regarding what self to actualise.

Agency shocks can be readily understood through the constraint lens. If someone is operating at their agency frontier and they experience a negative agency shock, this will likely have substantial impacts on their SWB. The same can be said for positive shocks that expand the capabilities frontier. For example, an aspiring professional athlete will experience an increase in their health as they train. Their SWB will consequently increase as they will become a more capable athlete. If they then suffer an injury their health will go down, tightening the constraint on their ability to actualise their athletic ideal self. Their SWB will decline.

An interesting issue that emerges from this example is the potential to substitute from one capability to another. Substitution is most feasible when an individual is not operating at their agency frontier across all the dimensions of agency and thus they have some slack in one dimension of their agency that they can start to utilise when a different dimension experiences a contraction. For example, someone with very high environmental quality who suffers a shoulder injury (health shock) and can no longer play racquet sports can substitute to hiking through that pristine environment. They may have avoided this activity previously because they enjoyed badminton marginally more than hiking, but now that badminton is precluded they choose to hike and experience only a minor decline in overall SWB.

\section{A formal specification}

Describing the wellbeing production function in mathematical terms is a tricky business. On the one hand, one wants to be specific and complete. On the other, one does not want to arbitrarily parameterise the model or make strong assumptions about things like additive versus than multiplicative relationships. These details should be left to empirical inquiry. In the interests of providing a thorough statement of how the different aspects of wellbeing fit 
together, the model outlined below does engage in arbitrary parameterisation and the like, but it must be stressed that the exact relationship between the variables will require further investigation. As emphasised in the introduction to this chapter, the value of this exercise is in sketching the entire landscape of wellbeing, not in providing a definitive portrait of that landscape.

The production function begins with the first-order structural equation (equation \#1):

$$
S W B=f(H, E, D)
$$

Where:

SWB $=$ subjective wellbeing

$\mathrm{H}=$ Hedonia

$\mathrm{E}=$ Eudaimonia

$\mathrm{D}=$ Despair

The second-order structural equations immediately follow (equations \#2, \#3 and \#4):

$$
H=f(P, N, S)
$$

Where:

$\mathrm{H}=$ Hedonia

$\mathrm{P}=$ Positive Affect

$\mathrm{N}=$ Negative Affect

$\mathrm{S}=$ Hedonic Life Satisfaction

$$
E=f(A, C, R)
$$

Where:

$$
\begin{aligned}
& \mathrm{A}=\text { Autonomy } \\
& \mathrm{C}=\text { Competence } \\
& \mathrm{R}=\text { Relatedness }
\end{aligned}
$$




$$
D=f(M, I, V)
$$

Where:

$\mathrm{M}=$ Meaning (nausea)

$\mathrm{I}=$ Identity (anguish)

$\mathrm{V}=$ Virtue (seriousness)

The parameters of emotional well-being $(\varepsilon)$ and nihilism $(\eta)$ are introduced next (equations \#4 and \#5).

$$
\varepsilon=f(E, D, a, \varphi)
$$

Where:

$0<\varepsilon<1$

$\alpha=$ attention

$\varphi=$ flow

E and D are eudaimonia and despair respectively.

$$
\eta=f(D)
$$

Where:

$0<\eta<1$

Emotional wellbeing parameterises hedonia in the following manner (equation \#6):

$$
H=f(P, S) \varepsilon-(1-\varepsilon) N
$$

To understand what is going on here, consider a case of high emotional wellbeing, say $\varepsilon=$ 0.8. In this case, the majority of the value of positive affect $(\mathrm{P})$ and hedonic life satisfaction $(\mathrm{S})$ are carried over to the total value of the hedonia $(\mathrm{H})$ variable. Meanwhile, $(1-\varepsilon)$ is 0.2 , which means that the overall impact of negative affect on hedonia is dampened by the individual's high level of emotional wellbeing. In the case of low emotional wellbeing, say $\varepsilon$ $=0.2$, the effects would be reversed. This is not a perfect model of emotional wellbeing because it does not capture dispositional effects. These would have to be modelled as part of 
a dynamic account of SWB. I do not present such an account owing to challenges associated with measuring SWB in a dynamic context that are discussed in chapter 10.

Nihilism parameterises eudaimonia in the following manner (equation \#7):

$$
(1-\eta) E
$$

So a high value of nihilism $(\eta)$ reduces the effect of a high score on eudaimonia (E). For example, if $\eta=0.7$, then $(1-\eta)=0.3$, and so only a third or so of the individual's eudaimonia score is carried over to their overall subjective wellbeing.

The next step is to substitute equations 6 and 7 into equation 1. From here on, I will assume an additive relationship between hedonia, eudaimonia and despair, and between the subvariables in each second-order equation, such as between positive affect and life satisfaction:

$$
S W B=\varepsilon(P+S)-(1-\varepsilon) N+(1-\eta) E+D
$$

What remains to be integrated are the parameters of personality and relative status.

Personality can be modelled as a function of the big five personality factors: openness, conscientiousness, extraversion, agreeableness and neuroticism. But for the sake of simplicity I will simply leave the effect of personality on SWB as a single variable, $\rho$.

Relative status effects (r) are a function of where the individual sits in some particular ranking that they care about and their propensity to care about that position:

$$
r=f(c, \delta)
$$

Where:

$0<\mathrm{r}<1$

$\delta=$ your position in the status distribution

$\mathrm{c}=$ propensity to care about one's status

Integrating personality and reference group effects into equation 8 yields:

$$
S W B=r \rho[\varepsilon(P+S)-(1-\varepsilon) N+(1-\eta) E+D]
$$

A few more parameterisations are worth mentioning. First, affect appears to exist on a bounded scale running from so much suffering that you pass out to so much ecstasy that you 
pass out (Kahneman 1999). Perhaps with direct chemical intervention we could achieve higher levels of affect, but in the natural course of life, affect has a maximum and a minimum. It is straightforward to impose such a structure on affect directly, though of course the numerical value of the upper bound is arbitrary:

$0<\mathrm{N}<10$

$0<\mathrm{P}<10$

The variables in the first-order structural equation can also be parameterised to only take positive values, so hedonia, eudaimonia, despair and SWB are all $>0$.

Second, SWB suffers from diminishing marginal returns to each of its component parts. This is known as the hedonic treadmill: we get used to sensations over time. The first heatwave of summer feels oppressively hot, but it is actually only a day in the high 20s (degrees Celsius). As summer drags on and our bodies acclimatise to the weather, even higher temperatures feel less oppressive. The declining intensity of such sensations was a foundational observation among classical economists like Edgeworth (Bruni and Sugden 2007). It was also one of the main areas of study in early hedonic psychology (Morris 1999, Frijda 1999). It is reasonable to conjecture that this observation can be extended to each of the variables in the wellbeing production function such that successive increments to scores produce relatively less SWB than previous increments. It is obvious enough that we acclimatise to material comfortsthese are very sensational in nature. But we also acclimatise to eudaimonia and the growth of meaning, values and identity. For example, consider an American professor at the peak of their career, married 25 years and still going strong, with kids who have recently departed for college and a network of old and deep friendships. This person is likely high in autonomy, competence and relatedness. Say they now meet a new colleague with whom they get along well. This improves their relatedness score. The effect would be marginal compared to say, the same event for a postdoctoral student trying to break into an existing network of researchers. Daniel Kahneman expressed similar sentiments after winning the Nobel Prize. It was a crowning achievement in an illustrious career, but precisely because was already sitting on the peak of a very high mountain, going one step further didn't seem to have that much of an effect (reported in Diener and Biswas Diener 2008, p. 153). One presumes that getting his first postdoctoral position may have provoked more of a surge in SWB. 
In neoclassical consumer theory, diminishing marginal returns to the consumption of particular goods is captured by exponential parameters. A similar approach seems appropriate enough for including diminishing marginal returns to hedonia, eudaimonia and despair. Each individual variable receives its own parameter: hedonia $(\tau)$, eudaimonia $(\theta)$ and despair $(\mu)$. This is to reflect that improvements to a neglected dimension of wellbeing will have relatively large effects compared to improvements to a dimension in which an individual is already strong. So for example, a materially impoverished but spiritually rich artist will likely get higher returns to wellbeing from an increase in their living standards (reflected in hedonic satisfaction) than they would from exhibiting 9 paintings in their next show compared to 8 . Incorporating these parameters for diminishing marginal returns into equation 10 yields the full wellbeing production function (equation 11):

$$
S W B=r \rho\left[(\varepsilon(P+S)-(1-\varepsilon) N)^{\tau}+((1-\eta) E)^{\theta}+D^{\mu}\right]
$$

Individuals looking to grow their subjective wellbeing do so subject to a constraint imposed by their level of agency. In the context of a developing country, this would need to be modelled. However, in the context of the developed nations where SWB advocates are pressing their policy suggestions it might sometimes be reasonable to assume that the capabilities constraint is meaningfully relaxed. Constrained people, such as the disabled, are arguably the exception rather than the rule in such contexts, whereas in developing countries this situation is reversed. The more pressing impediment to wellbeing in advanced nations is the dearth of information people have about what behaviours, values and goals would make them well. How individuals can overcome this information shortfall is discussed in chapter 9.

\section{Conclusion}

The benefit of specifying an integrated subjective wellbeing function is that it clarifies what the relevant variables and parameters are and indicates how they might fit together.

Researchers working on particular aspects of the function can then understand their work in relation to that of other scholars and can feed their findings, in particular their coefficient estimates, into the overarching model. In this way, disparate research streams can be brought together to discover the nature of the complex phenomenon of subjective wellbeing without many researchers having to work across the entire spectrum of the phenomenon. This is necessary in the context of SWB research because it has such a wide disciplinary scope and 
combines a large number of research streams that individually require disciplinary specialisation, impeding cross-disciplinary pollination.

This chapter presented a sketch of the wellbeing production function. Detailed explication of variables and extensive references to supporting literature were absent. In the next four chapters I provide this missing content. 


\section{Chapter 5}

\section{The capabilities constraint}

Even if we act to erase material poverty, there is another greater task, it is to confront the poverty of satisfaction - purpose and dignity — that afflicts us all...gross national product does not allow for the health of our children, the quality of their education or the joy of their play. It does not include the beauty of our poetry or the strength of our marriages, the intelligence of our public debate or the integrity of our public officials. It measures neither our wit nor our courage, neither our wisdom nor our learning, neither our compassion nor our devotion to our country, it measures everything in short, except that which makes life worthwhile. And it can tell us everything about America except why we are proud that we are Americans.

- Robert Kennedy, Remarks at the University of Kansas

\section{Introduction}

This chapter has two objectives. The first is to advocate for the idea that capabilities are a constraint that you try to maximise wellbeing within rather than being direct causes of wellbeing. The second is to justify the items in the capabilities constraint, namely income, health, education, enfranchisement and environment. The argument proceeds in the following manner. I begin by briefly discussing the notion of a budget constraint on utility in microeconomics. This idea directly informed the emphasis on income growth in early development economics. I argue that the more recent emphasis on capabilities and especially the way capabilities were operationalised in the Human Development Index, Millennium Development Goals, Sustainable Development Goals and the Sen-Stiglitz-Fitoussi Commission Report (2009) essentially involves broadening the budget constraint into a capabilities constraint. I draw on this operationalisation literature to justify the content of the capabilities constraint. 
The focus on capabilities in development policy is appropriate given the ethical obligations facing the governments that are the primary actors in the development policy space, particularly regarding paternalism. However, it means that development policy in advanced nations is somewhat distant from wellbeing. Expanding capabilities expands welfare, not wellbeing. Wellbeing is determined by functionings rather than capabilities. Functionings are related to the utility function in neoclassical microeconomics, not to the budget constraint. The production function theory of wellbeing extends this framework to associate the capabilities constraint with a wellbeing function much like the budget constraint is associated with a utility function. What functioning is best for one is often hard to figure out, and having more capabilities doesn't make it any easier. Indeed, the expansion of choice might actually make it harder to set yourself to one life. This is why existential malaise is a rich-person and first-world problem. I discuss empirical evidence that attests to this.

\section{Wellbeing in neoclassical economics}

The most basic building block of neoclassical economics is a model of the individual consumer trying to maximise their utility subject to their budget constraint. Utility here is defined in the vaguest terms as anything "good" for the individual. More specifically, it is defined mathematically as anything that can be associated with a plus sign (+). Disutility is represented by a negative sign (-). In introductory textbooks, the utility function takes the following "Cobb-Douglas" form:

$$
U=x^{\alpha} y^{\beta}
$$

Where $\mathrm{x}$ and $\mathrm{y}$ are two goods (or baskets of goods) and alpha and beta are parameters that capture the rate at which the marginal contribution of these goods to utility diminishes as consumption increases. Goods are assumed to have prices, $\mathrm{P}_{\mathrm{x}}$ and $\mathrm{P}_{\mathrm{y}}$. The individual's budget constraint is given by their own income. Expenditure must be less than or equal to income, and expenditure is given by the total quantities of $\mathrm{x}$ and $\mathrm{y}$ consumed multiplied by their respective prices. The budget constraint can thus be written:

$$
I=P_{x} x+P_{y} y
$$

The "consumer's problem", as it is called, is then given by the following objective function:

$$
\max U=x^{\alpha} y^{\beta} \text { s.t. } I=P_{x} x+P_{y} y
$$


An obvious inference that can be drawn from this model is that relaxing an individual's budget constraint will increase their utility—more income means more consumption which means more utility. The additional utility gained will diminish as consumption increases, but nonetheless there is a straightforward association between income and utility by way of greater consumption. Note though that it is the consumption of goods that provides utility, not income itself. The direct "causes" of utility are in the utility function.

The consumer's problem is the formal background to the traditional emphasis on income growth in development economics and the priority that was given to developing measures of GDP before any other metric of "wellbeing” (Masood 2016). Income growth is a central element in several structural changes that take place in the early stages of development, notably the agrarian transition and industrialisation (Foster-McGregor and Verspagen 2016, Thirlwall and Pacheco-López 2017). It also has tremendous power to improve the quality of life by financing health care, infrastructure, technology and other aids (Deaton 2013). Given this significance, it is only natural that development economists started with GDP. However, a series of objections were raised to its pre-eminence as a measure of wellbeing, development and social "progress" over the second half of the $20^{\text {th }}$ century. Four in particular stand out. These concerned issues of freedom, value, sustainability and distribution. I discuss each of these in turn below.

\section{Development as freedom}

Amartya Sen is famously associated with the argument that GDP is not a good measure of development because it is a poor proxy for whether people can actually do what they want to do and be who they want to be (Sen 1999a). While income measures whether someone can consume the goods they want, the notion of "goods" here is restricted to material goods that can be bought in commercial markets. Non-commercial goods, like dignity, career choice, living in a non-discriminatory society etc. cannot be measured by income, but are nonetheless important markers of wellbeing. Moreover, a society that was getting richer but providing fewer of these goods would not be regarded by many as "developing".

Sen proposed a richer notion of development than simply income growth. He called it "development as freedom" and emphasised that a society makes progress when it increases the possibilities open to its citizens (Sen 1999a). Income is an insufficient concept to measure such development. For example, if one is rich but disabled, one might struggle to fulfil one's 
wish of climbing Mount Everest despite having plenty of income. Or if everyone in society is getting wealthier but some opportunities are denied to an underclass of citizens on the grounds of caste, race, gender or sexuality then life is possibly not getting better for this underclass and it is contentious to suggest that society is making progress.

Sen (1999b) provides a formal framework for understanding this definition of development. He begins by distinguishing between wellbeing and advantage (ibid. p. 3). He defines wellbeing in terms of achievement: "how 'well' is his or her 'being'?" Advantage, on the other hand, "refers to the real opportunities that the person has, especially compared with others". Critically, "the opportunities are not judged only by the results achieved, and therefore not just by the level of well-being achieved". If a person with very restricted options nonetheless manages to live out the life that is uniquely wellbeing maximising for them, this does not mean that this person is free to be who they want to be and do what they want to do because they can basically only choose to be this one particular person - they have little freedom of choice (ibid. p. 9). Sen argues that the progress of a nation should not be judged by whether its citizens are able to find wellbeing in that society, but by whether they have a range of options available to them from which they can choose the one that will give them the most wellbeing.

This raises the important distinction between capabilities and functionings. Sen (1999b) approaches this distinction formally, but for ease of exposition I will dispense with the mathematics. A functionings are modes of being - they are the things one chooses to be and do. Capabilities refers to the option set of functionings: "[if] $\mathrm{Q}_{i}\left(\mathrm{X}_{\mathrm{i}}\right)$ represents the freedom that a person has in terms of the choice of functionings...Qi can be called the 'capabilities' of person i given those parameters[ $\mathrm{x}_{\mathrm{i}}$ ]" (ibid. p. 9). In other words, capabilities are the options someone has - all of their possible functionings - whereas someone's functionings are the options that they choose to actualise in reality. Wellbeing derives from how an individual values the particular functionings they choose to realise, and different functionings will thus give rise to different amounts of wellbeing to different people.

The most immediate operationalisation of Sen's theory was the adoption of the Human Development Index (HDI) as a complement to GDP by the UN and many other major development policy organisations. HDI incorporated education and health, measured by years of schooling and life expectancy, to attempt to better capture the capabilities people had (Hirai 2017). Education opens a range of doors unrelated to income, such as the ability to 
apply for jobs with specific educational requirements. Health is similar. For example, better health might allow one to be an athlete, or go on exhilarating hikes. A key reason for the early adoption of these variables despite education and health constituting a very limited proxy for human development was the ease of data collection.

To bring this discussion back to the model of wellbeing developed in chapter 4, capabilities are an expansion on the notion of a budget constraint, and functionings are an expansion on the notion of a utility function. In neoclassical consumer theory, the budget constraint captures the option set available to the individual. In Sen's notion of development as freedom, capabilities serve the same purpose, only the qualitative nature of the constraint is richer. It ceases to be entirely a matter of income, covering instead the broader notion of whether an individual can be who they want to be and do what they want to do. Who they then choose to be and what they choose to do are their particular functionings, and it is by these functionings that they derive utility. Functionings are characterised by qualities like consumption, career choice, family life, hobbies etc. Depending on the individual's preferences for these things, certain functionings will provide greater utility/wellbeing. Standard microeconomic theory about diminishing marginal returns to these particular characteristics likely still applies. For example, concentrating entirely on career and thus retaining little work-life balance could be expected to result in less total wellbeing for most people owing to greater diminution of marginal returns to career at such high levels of investment.

Critically, expanding the budget constraint to a capabilities constraint retains from neoclassical theory the notion that expanding a constraint doesn't cause utility or wellbeing growth. Rather, it opens more possibilities for consumption or functioning and it is these things that cause increases in utility or wellbeing. It is for this reason that I say that expanding capabilities increases welfare, but not wellbeing. Welfare is about access to wealth, resources, assets, opportunities, etc. hence its central place in theories of justice and the efficient allocation of scarce resources. Wellbeing is about hedonic and existential satisfaction. The two are connected, but not identical. Wellbeing requires the leveraging of welfare. If you don't know how to do that, acquiring more welfare won't increase your wellbeing much. In neoclassical theory it is assumed that the individual knows what they want to consume and that they optimise their consumption to maximise utility accordingly. Recent findings in behavioural economics show that this is not strictly true (Kahneman 2011). As Pareto 
emphasised, people need opportunities to learn what their preferences are before revealed preferences in consumer choice can be reasonably employed as a proxy for utility. The same basic logic holds for wellbeing. More capabilities means that people can access functionings they couldn't access before. However, unless people know which functioning is most appropriate for them, this increase in capabilities doesn't necessarily translate into greater wellbeing. Learning must first take place, which is where the coalescence of being comes in.

This notion of capabilities as a constraint is hard to appreciate in the context of a very tight capabilities constraint, such as that afflicting most people in poverty, because such a constraint seems to actively hurt you. For example, someone with a bung knee suffers as a result of this knee. It would appear to be a cause of ill-being in this case. Relaxing their health constraint by providing them access to high quality health care that can address their knee would then seem to cause an improvement in their wellbeing. There are some exceptional cases like this (especially in health), but for the most part, income, health, education, enfranchisement and environment only restrict or provide options that must still be realised and thereby converted into wellbeing. Alternatively, one can think of situations like the bung knee or authoritarian repression as cases of a negative capabilities constraint. This might be most appropriate in a developing countries context.

SW-B research is entwined with the positive psychology movement and is gaining traction mostly with the governments and NGOs of the advanced nations of the OECD. This is a different context to a developing country. There is little need to think about removing impediments like bung knees and authoritarian repression to people's functioning in these nations. The emphasis is instead on opening new vistas by helping people to attain tertiary education and peak physical fitness. Here the notion of capabilities as constraint rather than cause is straightforward.

There are two corollary points to be made here. First, the notion of capabilities as constraints rather than causes explains why further economic development in advanced economies like Australia, Denmark and Canada appears to have little impact on life satisfaction-people already have what they need to be who they want to be and do what they want to do. The issue is not one of resources or options but of self-knowledge. People's wellbeing is typically high in these countries, but sometimes they still can't attain full flourishing. A reason for this is they don't know how to leverage their capabilities effectively. They don't know which flourishings to actualise. 
This inference is corroborated by some empirical evidence (Ng and Diener 2014). Graham and Lora (2009) found that the most important determinants of wellbeing among the poor in Latin America are income and social support networks, while for the rich they are work and health. Similarly, Graham (2017) found evidence that the poor in America suffer from "bad" stress associated with desperation, while the rich suffer from "good" stress associated with goal-striving. These findings support the notion that the poor are heavily constrained in terms of their capabilities and must consequently fixate on life-sustaining activities. The rich, in contrast, have capabilities aplenty and can focus on more transcendental activities like career. Furthermore, Diener and Fujita found that resources such as health, wealth and attractiveness barely correlate with SW-B unless they are relevant to people's idiographic strivings (1995).

Second, if capabilities are a constraint on rather than a cause of wellbeing then the popular practice of looking for the determinants of wellbeing by running regressions with SW-B as the dependent variable and capabilities as explanatory variables bypasses a causal analysis. Direct causation is in a deeper substrate of analysis; one tied to preferences. It is unsurprising against this background that these regressions have low explanatory power, with $\mathrm{R}^{2}$ of around 0.15 (Clark et al 2018, p. 20). Several such investigations have been conducted to date and were certainly useful (Argyle 1999, Van Praag and Ferrer-i-Carbonell 2004). Improving their explanatory power further also has value. But if the field of SWB wants to move closer to a causal analysis, different data sets and models will be required, notably ones with preference information.

Datasets with such information are exceptionally rare at present and the ones that do exist typically contain information on consumption preferences rather than preferences over goals, life domains or other items directly relevant to SWB. When such preference questions do exist in a data set, such as in the Household Income and Labour Dynamics of Australia (HILDA) panel, they are not structured in a way that communicates trade-offs. For example, HILDA asks respondents to express how important income, leisure, family, religion and other items are to them on a scale from $1-10$. But respondents can say that they have an equally 10/10 preference for family, income, leisure etc. As a result, researchers cannot study which preferences are relatively stronger and whether these relative preferences play out in behaviour or affect the contribution of each of these domains to wellbeing. Given these data limitations, studying SWB with preference information is next to impossible at present. In chapter 10 I outline a methodology for more effectively collecting preference information in the context of SWB surveys. In the rest of the present chapter I discuss how capabilities have 
been operationalised and hence why I specify the capabilities constraint as consisting of income, health, education, enfranchisement and environmental quality.

\section{Nussbaum, political enfranchisement and the MDGs}

The next extension to the traditional economic emphasis on income growth concerned political enfranchisement. Around the time the Human Development Index was being implemented and refined in the 1990s, Sen was collaborating with Martha Nussbaum on closely related work concerning the gender dimensions of development. In Nussbaum's (2000) influential book, Women and Development: The Capabilities Approach, she argued for 10 "central human functional capabilities" that should underpin development policy. These were (pp. 78-80):

\section{Life expectancy}

2. Bodily health

3. Bodily integrity i.e. freedom from assault

4. Being able to use the senses, to imagine, think and reason

5. Emotions - being able to have attachments to things and people outside ourselves

6. Practical reason - being able to form a conception of the good and engage in critical reflection about the planning of one's life

7. Affiliation: being able to live with and towards others; also dignity

8. Respect for other species

9. Play

10. Control over one's environment, both political and material

Nussbaum was especially concerned about the special constraints facing women, particularly in developing countries, with respect to these central capabilities. Owing to the historical disenfranchisement of women they often face gendered obstacles that limit their freedom. For example, during and immediately after World War 2 in Europe women were explicitly paid lower wages than men for the same work, limiting their economic freedom (Summerfield 2012). In some Islamic countries and communities women are required to cover up or face opprobrium and criminal charges, which limits their cultural freedom to engage in a range of activities, like going to the beach (though the Burkini, an Australian invention, has improved things a little). Until recently, women in Saudi Arabia were not allowed to drive, severely constraining their mobility and freedom. Women were excluding from voting in many 
nations until recently, and are still ineligible to vote in the Vatican. These limited their political freedom. Similarly, they were excluded from a range of opportunities. Women were excluded were only allowed to graduate from Oxford University in 1920, even if they met all the academic requirements (Oxford University Archives 2007). They could not be members of the Berlin Philharmonic Orchestra until the 1908s. Finally, women's sexual freedom has often been curtailed through practices like enforced marriage and honour killings.

Nussbaum's arguments were operationalised somewhat in the Millenium Development Goals (MDGs). These included the eradication of poverty, universal primary education, the reduction of infant mortality and efforts to combat HIV/AIDS, malaria and other headline diseases. These correspond to the earlier focus on income growth and then education and health. The MDGs also targeted maternal health specifically and gender equality and the empowerment of women. This enfranchisement of women was pursued through 3 sub-gaols (United Nations 2015a):

- Eliminate the gender disparity in education between girls and boys at the primary, secondary and tertiary levels.

- Increase the share of women in wage employment in the non-agricultural sector

- Increase the proportion of seats held by women in parliaments worldwide

Broadly speaking, the emphasis here is on political freedom and non-discrimination, which is why I refer to this set of capabilities as pertaining to "enfranchisement". When someone is enfranchised in terms of their capabilities, it means that they are culturally, politically and legally at liberty to pursue their preferences. They may still lack the health, education, income and environment necessary to do so, but they are not actively disenfranchised by others, the community or the state.

\section{Sustainability and the SDGs}

The $7^{\text {th }}$ millennium development goal was to ensure environmental sustainability. This objective has increased in prominence in recent years and efforts to achieve it have accelerated. The clearest demonstration of this is the replacement of the millennium development goals with the sustainable development goals (SDGs: United Nations 2015b). These extend earlier initiatives born under the HDI and MDGs. For example, there is now an 
objective to achieve peace, justice and strong institutions, which speaks to political enfranchisement. More prominently, the SDGs place greater emphasis than in the past on environmental issues. There are new goals concerned with sustainable cities and communities, climate action, responsible consumption and production, organic life on land and in the water, and infrastructure development. This strong emphasis on environmental capabilities in the contemporary development literature speaks to the need to include environmental factors in the capabilities constraint.

The most obvious way that environment constrains wellbeing is in negative terms through the effects of pollution, congestion and overcrowding, which make life unpleasant and difficult. But what about in places where environment in terms of air and water quality and population density is good, like Australia or the United Kingdom? Here we come across other issues that relate more subtly to the notion of capabilities. For example, both Australia and the UK have problems around good quality, affordable housing that is proximate to opportunities like interesting, well-paid work, effective schools and enjoyable leisure activities. Owing to a range of policy settings that constrain sensible and equitable real estate development (Daly and Coates 2018), many young Australians face a trade-off between living in affordable, pleasant housing that is far from many opportunities or living in run-down or poorly built, often crowded neighbourhoods close to opportunities. These less pleasant neighbourhoods can also feature other environmental bads like high crime rates, noise pollution and limited amenities like parks. Some of these trade-offs are unavoidable to some degree, at least without major technological changes around things like telecommuting - you can't live both next to a major commercial enterprise and a national park, for example. Others, however, are surmountable through advances in urban planning and design, as advocated by the SDGs and as explored in the burgeoning literature on wellbeing and geography (Glaeser 2012, Arampatzi et al 2018, Okulicz-Kozaryn 2015).

\section{Distribution and social capital}

Before concluding, the issues of welfare distribution and social capital must be addressed and reasons given as to why they are not factors in the wellbeing production function or the capabilities constraint. Alongside environmental outcomes, the SDGs also target reductions in inequality in society. Such inequality, particularly when considered through the lens of just distribution, is a long-running theme in welfare economics (see, for example, Atkinson 1970, 
Fleurbaey and Maniquet 2011). Why then do I not include some account of the distribution of capabilities in the capabilities constraint? Similarly, some recent work on measuring development has promoted the so-called "four capitals" framework, which takes in built, human, environmental and social capital (Arrow et al 2012, Costanza et al 2012). I have accounted for the first three of these in income, education and health, and environmental capabilities, but political enfranchisement is insufficient to capture the full scope of social capital. Absent are things like networks, cultural markers and the ability to blend into a particular cultural group, like the ruling class. Why do I not account for social capital in the capabilities constraint?

The reason why I have not included these items in the capabilities constraint is because they are included elsewhere in the wellbeing model. Inequality is incorporated through reference group effects and low-rank aversion. This is more appropriate than including it in the capabilities constraint because being of low rank in a reference group appears to exert a direct impact on wellbeing, with the severity varying by person. Inequality is thus a matter for the wellbeing function, not the capabilities constraint. Social capital is picked up through the relatedness variable. Individuals attempt to adopt the cultural markers of particular networks in order to join them. Success in this endeavour, which depends in part on whether the group's identity is self-concordant with the individual's identity, results in the ability to blend into that group. What remains unaccounted for in terms of social capital is picked up by political enfranchisement. Of particular note here is the notion of equality of opportunity. If the ruling class, for example, is less a meritocratic group and more a matter of hierarchies enforced by laws, institutions and discrimination, such as was the case under the apartheid regime in South Africa, then individuals without social capital do not have the capability to advance themselves. But it is precisely such factors that are captured in the political enfranchisement aspect of the capabilities constraint, so no additional variable is required.

\section{Conclusion}

Capabilities should be thought of constraints on wellbeing rather than direct causes of it. It is functionings that give rise to wellbeing. Different functionings will suit different people, and the completion of a difficult process - the coalescence of being - is required to discover which functionings are best for you. Given this delineation, capabilities should not be entered directly into the wellbeing function. By extension, it is quixotic to continue to search for the 
determinants of wellbeing in correlations between SWB indicators and elements of the capabilities constraint, especially in advanced nations wherein the capabilities constraint is largely relaxed. If we want to discover the causes of wellbeing we need to dig deeper.

Capabilities can be effectively operationalised using income, health, wealth, political enfranchisement and environmental quality. What this limited taxonomy loses in nuance and comprehensiveness it more than makes up for in parsimony and ease of use. This is important in the context of the empirical investigation of wellbeing, which has historically been plagued by data availability issues. Operationalising capabilities in this way keeps the production function of wellbeing connected to the development economics literature, to development policy in practice, and to the relatively rich data collection efforts associated with these. 


\section{Hedonia}

It is impossible to live a pleasant life without living wisely and well and justly. And it is impossible to live wisely and well and justly without living a pleasant life.

- Epicurus

The chapter has three objectives. First, to demonstrate that hedonia is in fact distinct from eudaimonia and despair. Second, to describe the nature of hedonia based on theoretical arguments and empirical evidence. And third, to discuss various techniques that are useful specifically to the promotion of hedonia, as distinct from eudaimonia, despair or wellbeing in general.

On the first subject, I provide statistical evidence from SWB research that "experienced" wellbeing, which refers in that literature to affect, is distinct from "evaluated" wellbeing, which in that literature refers to life satisfaction. In my model I break evaluated wellbeing down into a more fine grained taxonomy, but the point stands that one's emotional state in the moment is a distinct dimension of wellbeing. I add to this account using philosophical arguments from Haybron $(2001,2008)$ regarding an individual's emotional disposition. This extends the emphasis on experienced wellbeing in SW-B research to encompass emotional wellbeing more broadly. Finally, I present some of my own philosophical arguments regarding the separability of what I call "hedonic satisfaction" from what I call "existential satisfaction". The former is an evaluation of how pleasant one's life is and properly belongs in the hedonia dimension. The latter refers instead to how fulfilling and valuable one considers one's life and thus encompasses the eudaimonia and despair dimensions.

Having demonstrated the independence and veracity of hedonia I then describe its nature. I draw in particular on the hedonic psychology literature. Here I discuss hedonic adaptation, the peak-end rule and other cognitive biases or quirks associated with affective systems, the 
neuropsychology of emotion and the determinants of emotional resilience. I also discuss findings from experience sampling studies of what behaviours people find most and least enjoyable.

In the final section of this chapter I canvass a range of techniques that can improve hedonia. These include positive activity interventions like gratitude and humility, the hedonic adaptation prevention model, "bringing in the good", mindfulness, flow, cognitive behavioural therapies and crude hedonism. I also explore some practices that will encourage negative affect, including learned helplessness, rumination and leaning into the hedonic treadmill. This follows through on the claim in chapter 3 that wellbeing research needs to consider not only what wellbeing is but also how it can be achieved. Managing hedonia is also a sensible topic to cover given evidence that happiness is associated with a range of better life outcomes, including greater longevity, strong immune systems, less hypertension and higher productivity (Diener and Biswas-Diener 2008, Jacobs-Bao and Lyubomirsky 2013).

\section{The distinctiveness of hedonia}

SW-B scholarship has done a great deal of statistical work demonstrating that affect is distinct from life satisfaction. The main evidence used to support the claim is that events and changes in circumstances tend to affect one or the other variable (experienced wellbeing or evaluated wellbeing) differently. There is voluminous evidence for this (Lucas et al 1996, 2003, Kahneman and Deaton 2010, Diener et al 1999, Stutzer and Frey 2008). A second piece of evidence used to promote this claim is that experienced and evaluated wellbeing have different correlates (Luhmann et al 2012, Diener et al 2010). For example, child-rearing tends to lower experienced wellbeing, particularly by increasing negative affect, while also increasing evaluated wellbeing (Powdthavee 2008). Recreational use of hard drugs would have the opposite effect. Intuitively, it is not hard to imagine a range of activities that would improve evaluated wellbeing, especially in the long run, but reduce experienced wellbeing. For example, the last few months of a $\mathrm{PhD}$ are an arduous, stressful time that decreases experienced wellbeing, but growing closer to the goal of submitting your dissertation increases evaluated wellbeing. It is important to separate these items out if we are to understand the causal structure of wellbeing and why people make particular trade-offs across wellbeing dimensions in their life choices. 
There is also substantial evidence that negative and positive affect should be entered as two separate items in the utility function, rather than as a single "mood" or "happiness" variable going from low to high (Stone and Mackie 2013, p. 39). This observation goes back at least to Bradburn, (1969) who found that positive and negative affect are not opposite ends of a single spectrum, but are rather distinct from each other. His findings have been replicated multiple times (Kahneman et al 1999, Diener et al 1999, Gere et al 2011). The distinctiveness of positive and negative affect can be grasped intuitively by considering that someone can be experiencing both kinds of affect simultaneously. For example, someone might be stressed about an impending deadline but also happy to be catching up with an old friend. Curiously, positive affect is qualitatively unidimensional (it is usually described as "joy") whereas negative affect has multiple qualitative dimensions like stress, anxiety, depression and bereavement (Argyle 2001, p. 10).

The empirical evidence for these two propositions - that experienced and evaluated wellbeing are different and that negative and positive affect are distinct - is strong enough that a high level panel of the National Academies of the United States concluded:

\footnotetext{
Although life evaluation, positive experience and negative experience are not completely separable - they correlate to some extent - there is strong evidence that multiple dimensions of SW-B coexist. Experienced well-being is distinct enough from overall life evaluation to warrant pursuing it as a separate element in surveys; their level of independence demands that they be assessed as distinct dimensions (Stone and Mackie 2013, p. 4).
}

The evidence from SW-B scholarship justifies including positive affect and negative affect as separate terms in the wellbeing function and distinguishing an affective dimension of wellbeing from an evaluated dimension. However, more is required to justify the separation of hedonic life satisfaction from other dimensions of evaluated wellbeing, and the inclusion of the emotional wellbeing parameter. I turn to these items next.

\section{Hedonic versus existential satisfaction}

SW-B research uses a single-item in its investigations of evaluated wellbeing-life satisfaction. To better understand the component parts of this global evaluation it is helpful to break it down into concepts that are theoretically separable. One such breakdown is to 
differentiate between judgements of whether life is pleasant versus judgements of whether life is fulfilling and valuable (See Annas 2004, Deci and Ryan 2008, Waterman 2008). The two are not mutually inclusive. Separating the two out can help us to grasp intuitively how different preferences and circumstances can drive wellbeing dynamics.

Some examples will help illustrate the difference between hedonic and existential satisfaction. Earlier in chapter 4 I gave the example of a middle-class university student in an OECD country afflicted with nihilism. They are physically healthy, secure, with a bright future ahead of them. They have had every advantage in life, and can probably continue to live in good conditions with food, clothing and entertainment in perpetuity. They certainly live in such circumstances now. However, they also suffer from ennui-a kind of existential boredom. They lack seriousness and purpose, and they don't know who they are or who they want to be. This kind of person would score high on hedonic satisfaction and low on existential satisfaction. If you asked them about their life they might say they "can't complain". Or "everything is fine but you know, there's just something missing".

The opposite case is exemplified by a genuine freedom fighter in jail or hiding in the jungle. They have a cause that they believe in that gives them seriousness, meaning and identity. They have comrades who nourish their sense of relatedness. They are actively fighting for autonomy and, if the revolution is going well, they will feel competent. But their day-to-day circumstances might be extremely unpleasant, and so their hedonic satisfaction will be low.

Two other examples would be someone with a high-paying but ultimately lame job, and the archetypal long-suffering artist, beloved by philosophers everywhere. The former would have a pleasant but hollow life, while the latter's life would be a struggle but a passionate one.

To understand why appreciating these different dimensions of evaluated wellbeing is important for developing a causal understanding of wellbeing, we can return to the earlier example of someone nearing the completion of their $\mathrm{PhD}$. Assuming this person is an Australian $\mathrm{PhD}$ student on a typical scholarship, this person is likely presently low in affect owing to the stress of writing up their dissertation, but moderately high in hedonic life satisfaction owing to their middle-class income in a highly developed nation. Their overall hedonia is thus modest to good. Meanwhile, their existential satisfaction is perhaps slightly better, relatively speaking. They have a clear goal in mind, a collegiate community (hopefully), they work on what they want to work on and, assuming they are in fact nearing completion, they feel increasingly competent. After the completion of the $\mathrm{PhD}$, this situation 
is likely to change. We might expect affect to improve markedly as the stress ebbs away. Hedonic satisfaction will also likely increase as the individual now moves back into the conventional workforce and receives a pay rise. The impact on existential satisfaction is less clear-cut, but likely positive. Having a $\mathrm{PhD}$ in hand is a constant reminder that you are competent and accepted by your peers. However, the individual may lose a major source of meaning after submitting and may have to find paid work where they have less autonomy.

The examples above demonstrate not only the possibility of differentiating hedonic and existential satisfaction in theory, but also the usefulness of doing so for understanding the causal origins of changes in wellbeing. Sticking with life satisfaction as a global metric for everything to do with evaluated wellbeing is advantageous in that it facilitates quick data collection and provides a straightforward point of reference. However, to effectively investigate and understand what provokes changes in life satisfaction we need to specify a more disaggregated concept. This will allow us to appreciate nuances.

It should be noted that there is empirical evidence to suggest that hedonic and existential satisfaction are two different constructs. Clark and Senik (2011) report a correlation between life satisfaction and four different aspects of eudaimonic wellbeing of between 0.25 and 0.29 . Diener et al (2010) report a correlation of 0.62 between their psychological well-being scale — which is supposed to reflect eudaimonic aspects of well-being — and life satisfaction scale responses. The correlation between their psychological well-being scale and measures of positive and negative affect is 0.62 and 0.51 respectively. Huppert and So (2013) found a correlation of 0.34 between "flourishing"-which takes in aspects of eudaimonic wellbeing - and life satisfaction in European social survey data. These studies are not reporting exactly the split I am describing between hedonic and existential satisfaction. However, the finding that eudaimonia (which includes meaning and purpose in most of these studies) is only a component of life satisfaction suggests that hedonic and existential satisfaction are distinct.

\section{Emotional Wellbeing}

Recall from Chapter 4 that emotional wellbeing is a parameter that affects hedonia. It refers not to one's emotional state, which is captured by the positive and negative affect variables, but instead to one's emotional disposition — how inclined you are to react positively or 
negatively to emotional shocks. Individuals low in emotional wellbeing will have weaker and more transient responses to positive shocks and stronger, longer-lasting responses to negative shocks. Vice versa for people high in emotional wellbeing. It is important to distinguish between affective state and disposition to appreciate the full causal structure of hedonia. To illustrate, consider that some Buddhists like Mathieu Ricard (2014) are both low in negative affect and less likely to experience sustained negative affect because they have trained their minds to navigate away from such feelings (Dorjee 2014). I discuss a range of techniques that bear on emotional wellbeing, like mindfulness and bringing in the good, below. The extent to which the individual practices these techniques, whether with full cognizance of what they are doing or inadvertently, is captured by the "attention" variable, of which emotional wellbeing is a function.

Emotional wellbeing is also a function of eudaimonia and despair. Self-determination theory notes that individuals whose lives do not nourish their basic psychological needs for autonomy, relatedness and competence are more likely to manifest symptoms of depression, anxiety and poor mood and less likely to present with symptoms of psychological wellbeing (Roth et al 2017, Ryan et al 2016, Laurin et al 2015, Britton et al 2014, Weinstein and Ryan 2011). Similarly, empirical research in the area of logotherapy - a school of clinical psychology that investigates the role of meaning in psychological wellbeing-finds that people low in meaning and purpose are more likely to be depressed, anxious and despondent (Weinstein et al 2012, King and Hicks 2012, Ryff 2012, Slattery and Park 2012). Recent statistical work with large samples by SW-B scholars finds inverse results for people high in meaning and purpose (Yalçin and Malkoç 2015). Research in terror-management theory finds that people who feel a lack of seriousness concerning their values become distressed and anxious (Solomon 2012, Salzman and Halloran 2004, Dechesne and Kruglanski 2004). Some research on religion suggests inverse effects for people who feel deeply connected to their values (Emmons 1999). Finally, work in developmental psychology suggests that individuals struggling to define themselves, like adolescents, are more prone to depression, anxiety and low mood and that these symptoms are alleviated as these individuals learn who they are (Summer et al 2014, To and Sung 2017). 


\section{The Nature of Hedonia}

Hedonic judgements of present affective state and related matters like felt pleasure and pain are characterised by a range of curious cognitive phenomena. These include the peak-end rule, reference points and their interactions with prospecting, the focusing illusion, adaptation, the hedonic treadmill, homeostatically protected mood, and the mood effect on global evaluations. I review these matters in this section drawing on literature from the last three decades of research in hedonic psychology (Kahneman, Diener and Schwartz 1999).

\section{The Peak-End Rule and Hedonic Experience}

Kahneman (1999) laid out the core features of hedonic experience according to then state-ofthe-art empirical evidence. He differentiated between "subjective happiness", which "is assessed by asking respondents to state how happy they are", and "objective happiness", which "is derived from a record of instant utility over the relevant period". An example of an objective happiness recording instrument is to ask patients undergoing a colonoscopy to report their pain on a scale of $1-10$ every minute.

Some of the most interesting findings from early hedonic psychology concerned the discrepancy between how people experienced pleasure and pain and how they remembered it. For example, the main determinants of how painful (pleasurable) people remember an experience are the peak of pain (pleasure) associated with that experience and how much pain (pleasure) they felt at the end of the experience (Schreiber and Kahneman 2000, Frederickson 2000). When a better end was added to a painful experience, people seemed to prefer a long event with more total pain than a shorter event with a more painful finish (Kahneman et al 1993). Similarly, people would recall more negatively events with high peaks of pain even if those events were shorter overall (Do et al 2008).

Reference points, focusing illusions and loss aversion:

Alongside his work on hedonic psychology, Kahneman is well known for his work on prospect theory (Kahneman and Tversky 1979, Tversky and Kahneman 1986). The two streams of research intersect when it comes to the importance of reference points for hedonic evaluations. Prospect theory holds that "the carriers of decision utility are gains and losses 
relative to a reference level, which is often the status quo" (Kahneman 1999, p. 17, emphasis added). What this implies is that people's assessments of how happy or sad they will be made by a change in circumstances is coloured by their present circumstances:

A given state can be assigned quite different utilities depending on the state that preceded it, and quite different states can be assigned approximately the same utility if they represent the same change relative to the reference level.

Kahneman (1999) uses the example of paraplegics to illustrate the implications of prospect theory in this context. Most people are surprised to learn that people who become paraplegics appear to largely recover pre-accident levels of life satisfaction within 2 years. Kahneman posits that this is because people focus on the event of becoming a paraplegic, where the reference point is being able-bodied, and pay insufficient attention to the experience of being a paraplegic, where the reference point is being a paraplegic. While the research on paraplegics is questionable in light of measurement issues discussed in chapter 10 and the high willingness to pay among disabled individuals to reverse their conditions (Loewenstein and Ubel 2008), it illustrates neatly why prospect theory is relevant to evaluations of wellbeing.

Two related ideas from prospect theory are worth mentioning here: loss aversion and focusing illusions. Loss aversion refers to the fact that people tend to weight losses more than gains when making decisions. A famous example is that people want more money to sell an item than they are willing to offer to buy the same item. In the context of life satisfaction, individuals are hypothesised to weight losing what they have more substantially than making further improvements in their quality of life.

Focusing illusions are items that become salient during judgements of happiness that then bias those judgements by anchoring responses. A famous example is that citizens of the American mid-West anticipated that they would be happier if they lived in California because the weather there is better (Schkade and Kahneman 1998). However, residents of California and the Mid-West reported similarly high levels of SW-B. It would seem that California has some draw backs - perhaps high house prices and long commutes, which offset the good weather effect. The findings of this study and at least one other famous study of focusing illusions (Kahneman et al 2006) might fall foul of scale-norming and ceiling effects, which I discuss in chapter 10. Other studies, however, are more robust. For example, Strack et al (1988) found that priming respondents to think about how many dates they had been on 
recently caused them to dramatically re-evaluate their life satisfaction relative to a control group. Similar anchoring effects have been observed for priming people with health issues or the quality of their marriage (Schwarz et al 1991, Smith et al 2006).

In the model presented in chapter 4, these phenomena are captured by reference group effects. This is insufficient to capture the full complexity of cognitive biases about SW-B, but my interest is in specifying a wellbeing production function, not a cognitive evaluation function for SW-B. I go into some detail in chapter 10 on why we are quite far away from understanding the later in the context of SW-B.

\section{The hedonic treadmill, adaptation and homeostatically protected mood}

A major topic of inquiry in SW-B research is the adaptation hypothesis. This is the idea that people get used to changes in their circumstances such that the effects of those changing circumstances on wellbeing fade with time and people return to a long run "set point" of SWB. Adaptation is certainly what we see in life satisfaction panel data. Oswald and Powdthavee (2008) find substantial adaptation to disability, and Powdthavee and Stutzer (2014) find similar effects for income growth. Other studies have found relatively rapid adaptation to childbirth and marriage and slower adaptation to the death of a spouse and divorce (Clark et al 2008a, Dyrdal and Lucas 2013, Lucas et al 2003, Stutzer and Frey 2006, Specht et al 2011). Some circumstantial changes are severe enough to have lasting effects, but some degree of adaptation is still typically observed. These include cosmetic surgery and unemployment (Clark 2006, Powdthavee 2012). As I have already flagged a few times, there are residual questions about the reliability of scale measures in the context of dynamic change owing to issues of scale-norming, but leaving those aside for now, there seems to be a solid body of evidence to suggest that adaptation is real.

Set point theory is a relatively more recent addition to the adaptation hypothesis that posits a baseline level of SW-B (in terms of both affect and life satisfaction) that people return to as they adapt to shocks (Lucas et al 2004, Lucas and Donnellan 2007, Lykken 1999, Heady 2010, Heady et al 2014). This set point is a kind of average level of SW-B that is apparent when looking at life satisfaction scale data. There is some evidence that set-points are substantially genetically determined, and that they vary substantially with personality (Lykken and Tellegen 1996, Røysamb et al 2014). In particular, extroverts tend to have 
higher set points than introverts and other people high on neuroticism. Finally, there is some evidence that set points, at least those for mood, are homeostatically protected (Cummins et al 2014). That is to say, neurochemistry has evolved to bring us back to a mild level of background positivity. Being depressed or ecstatic for sustained periods of time is maladaptive: depressed people lack motivation and ecstatic people are easily distracted from tasks required to maintain life. Consequently, we are wired to feel such things only temporarily, while the rest of the time we feel pretty good, naturally (Cummins 2014).

Alongside homeostasis, adaptation is conjectured to be driven by 2 forces: changing reference points and the hedonic treadmill (Layard 2005). Relative status effects, especially those associated with where in a particular ranking an individual sits, have been shown to exert a strong effect on people's satisfaction with their circumstances (Frijters and Mujcic 2013, Boyce et al 2010, Brown et al 2008, Bhuiyan 2018). One might think that an improvement in objective life circumstances would result in an improvement in SWB. However, structural changes in life circumstances can often change people's reference points such that they now assess their position within some new ranking. For example, when an individual from a working class background becomes the CEO of a mid-sized company, we might intuitively expect their high income rank to result in high SWB. However, it is possible that this individual will now judge their income by where it sits in a ranking comprised only of other CEO salaries, not the salaries of the general population. Consequently, their SWB might actually decline. This might explain why lottery winners seem to adapt rapidly to their newfound wealth (Brickman et al 1978) despite some persistent positive effects (Lindqvist et al 2018). Another commonly conjectured manifestation of such changing reference points is rising aspirations. The idea here is that as people achieve long-held visions of success, they develop new aspirations that cause them to acclimatise to their existing achievements (Sheldon and Lyubomirsky 2012).

It should be noted that the psychological processes underpinning status anxiety can be treated with cognitive discipline (Lyubomirsky and Ross 1997). That is to say, while there may be an unconscious, natural inclination among humans to care about status and thus to acclimatise to new income and new reference points, you can make conscious choices to avoid such acclimatisation.

The Hedonic Treadmill is about getting used to sensations rather than circumstances, though sensations here is meant broadly such that it includes, say, the prestige associated with a more 
senior position at work. Kahneman (1999) uses comparisons to the psychology of colour and absolute length to explain treadmill effects. In experiments pertaining to people's perception of the intensity of colour, people's sensory mechanisms adapt to the brightness and richness of hues and experience them less intensely under repeated stress. In length experiments, people are given two lines in each phase of an experiment: short and medium, and then medium and long. In the first phase, people will describe the medium line as, for example, "neither long nor short". In the second, they will describe it as, for example, "short" or "very short". This is a context effect. The different outcomes in these two experiments are "produced by different processes: colour adaptation reflects a change in the sensory mechanism, whereas the context effect observed in size judgements is derived by the requirements of effective communication" (ibid. p. 11). The hedonic treadmill is about changes in the sensory mechanism. It is like acclimatising to a hot bath-scalding at first, then pleasant. The temperature of the water doesn't change and neither does the individual's reference point. Instead, their sensory instruments adjust.

\section{Hedonic psychology and economics}

Economists have played an increasingly prominent role in SW-B, typically alongside hedonic psychologists. Their efforts have been focused predominantly on the relationship between SW-B and income and unemployment, though more recently they have also become interested in the role of emotional factors like hope, optimism and stress in economic decision making (note that optimism can be captured by the personality trait in the wellbeing production function: see Sharpe et al 2011). These are all important issues for the wellbeing production function. Crude hedonism is largely a function of disposable income to spend on pleasantries and frivolities. Work is a major source of competence, meaning and relationships. Affective signals, including stress, play an important role in the coalescence of being.

Graham (2017) provides a thorough review of the existing literature on the relationship between hope, optimism, stress and SW-B and economic outcomes (see also De Neve and Oswald 2012, De Neve et al 2013). People who are more hopefully and optimistic have lower discount rates and invest more in the future than pessimistic and anxious people. They also appear to be more resilient to negative shocks, both in terms of their behaviour and their SW- 
B (Favara and Sanchez 2017). This finding parallels findings in the study of learned helplessness and learned optimism, which gave birth to positive psychology (Seligman 1975).

Poor people and rich people appear to experience different kinds of stress, on average. The poor suffer from "bad stress" associated with insecurity and desperation (Graham and Chattopadhyay 2010). They rely on social networks to mitigate these effects. In contrast, wealthy people experience "good stress", which is associated with goal pursuit and striving more generally (Graham and Lora 2009).

Extending earlier work on loss aversion, Graham (2017) finds that previously rich, typically white, typically semi-rural communities in America that have declined as a result of deindustrialisation express large declines in SW-B. In contrast, historically poor, typically black, typically inner-city communities are optimistic about the future in America and report relatively high SW-B despite being poorer on average than many of the declining white communities.

Finally, Graham finds that inequality can have positive effects on optimism, investment and work ethic if it is perceived to be the result of genuine economic opportunities (Graham 2011). In this case, people believe that hard work will see them get ahead (Graham and Nikolova 2015). By contrast, inequality has a dampening effect if it is perceived to be a function of inequality of opportunity and extractive institutions. If people think the deck is stacked against them and there is no way to get ahead, they will exert relatively less economic effort.

The effects of unemployment have already been touched on: unemployment results in large decreases in SW-B that are not entirely adapted to over time. Sustained unemployment also seems to have a scaring effect. Even after the long-term unemployed return to work, their SW-B stays somewhat depressed (Knabe and Rätzel 2011).

Research into the relationship between income and wellbeing deserves some longer discussion, if only because of the central place it occupies in the happiness economics discourse. The seminal paper in this literature was Easterlin's 1974 study of the relationship between life satisfaction and GDP using the World Values Survey. He found that while income explained differences in satisfaction within countries, it did not explain differences between countries. This finding became known as the Easterlin Paradox and it animated SWB scholars for several decades. Easterlin speculated that this might be due to adaptation, 
specifically reference group effects, where rich people within countries counted themselves wealthy, but poor people in wealthy countries did not compare themselves to even poorer people in other countries. The Easterlin Paradox was a major driver of early research into reference groups, rising aspirations and adaptation in early SW-B scholarship.

Research in the late 2000s took a lot of the wind out of the Easterlin Paradox's sails. Most importantly, Stevenson and Wolfers (2009, 2014), using updated World Values Survey data, demonstrated that there is a consistent linear relationship between the log of income and life satisfaction, and that a satiation point for income does not exist. As income grows life satisfaction continues to improve. Stevenson and Wolfers argued that the Easterlin Paradox was an artefact of bad data from the early waves of the World Values Survey. These waves typically suffered from sampling bias associated with only interviewing wealthy people in poor nations (only they had telephones) and not including enough poor nations.

Stevenson and Wolfers do concede that the relationship between income and happiness is linear only on the $\log$ scale, meaning that the richer you get, the more money you need to get an increase in your life satisfaction. This means that while there is no satiation point, there quite quickly comes a point where there may be much cheaper or easier ways of getting happier than earning more money. This aligns with the arguments of the preceding chapter, which argued that further relaxing an individual's capabilities (or budget) constraint beyond the point where they already have the agency they need is unlikely to have much effect on their life satisfaction. Stevenson and Wolfers also do not refute either the adaptation hypothesis or reference group effects.

Easterlin has recently presented fresh evidence in favour of the idea that economic growth has limited effects on wellbeing. In this case, the evidence comes from China, where spectacular economic growth from 1990 to 2015 does not seem to have translated into improvements in life satisfaction (Easterlin et al 2017). These are striking findings, but the methodology leaves a lot to be desired. Like most research in happiness economics, the analysis is purely correlational. It is quite possible that economic growth in China has had a tremendous positive impact on wellbeing that is offset by other factors, like rising levels of pollution and political repression. The analysis also relies on scale responses in a context where ceiling effects and scale-norming are major concerns.

A final important angle on the relationship between economics and SW-B is new research exploring changes in SW-B as a result of macroeconomic fluctuations (Boyce et al 2018). In 
particular, economists have recently begun exploring the differential impacts of recessions and booms on SW-B (De Neve et al 2018). Initial findings suggest that recessions have larger negative effects on SW-B than booms have positive effects. However, these results must be treated with caution as the data sets used pertain to advanced economies where ceiling effects might cloud responses.

\section{Results from experience sampling and time use surveys}

This section briefly reviews our current understanding of what activities affect mood and emotional responses. Which are core features of the hedonia variable. The findings are drawn from studies using experience sampling (Stone et al 1999, Hektner et al 2007) and day reconstruction methods (Kahneman and Krueger 2006). The former involves paging respondents over short, regular intervals (hourly, for example) and having them report their mood and current activity. The latter asks respondents to describe their mood and activities over the course of the previous day. Both methods are considered valid and effective measurement tools (Schwarz et al 2008).

Kahneman et al (2004), using the day reconstruction method and a large sample of women in Ohio, found that people had the highest levels of affect while engaged in sex, socialising, relaxing, eating, exercising, practicing religion and watching television. They reported relatively lower levels of affect while talking on the phone and napping, and while engaged in chores including cooking, shopping and computer tasks. The lowest levels of affect were reported for housework, childcare, commuting and working. Similar results are reported by Argyle and Lu (1990).

White and Dolan (2009) and Dolan (2014) have recently used experience sampling methods to extend this analysis by distinguishing between activities that give pleasure and those that give purpose. They find that some relatively less pleasurable activities like household chores and working are high in purpose, while some pleasurable activities like watching television are low in purpose. Two items that were relatively high in both pleasure and purpose were spending time with kids and volunteering activities. White and Dolan's research is a very interesting extension of Aristotelian ideas regarding the differences between hedonia and eudaimonia because White and Dolan focus on the experience of pleasure and purpose in the 
moment. Most philosophical and psychological accounts consider these two dimensions of wellbeing from the life evaluation standpoint instead.

A final topic that must be mentioned briefly here is the notion of flow, which is studied using experience sampling methods. This is a feeling of being "in the zone" or "lost in the moment" that transpires when individuals are engaged in high challenge activities with immediate, high quality feedback that they are skilful at and intrinsically motivated to undertake (Csikszentmihaly 2002). Flow is a very positive state, but it is misleading to describe it as a positive emotion because consciousness tends to dissolve during flow and the individual doesn't really feel anything - it is a state of enjoyable focus rather than a mood. Flow is something that can permeate your entire life, but is typically studied through the lens of particular activities, like sport or practicing a musical instrument (Csikszentmihaly ibid. Bakker 2005). I will discuss flow in the broader sense and in greater detail in chapter 9 on the coalescence of being.

\section{Techniques for achieving hedonia}

In the remainder of the chapter I survey a range of techniques for improving hedonia, in particular one's mood. For this I draw largely on the literature from hedonic psychology and cognitive-behavioural therapies. Some of these techniques, notably mindfulness, have more general benefits, but they are not meta-strategies for achieving holistic well-being. For the most part, these techniques are what I call "methods of mood management". In the closing passages of the chapter I discuss why mood management might often be insufficient for achieving deep, holistic and resilient wellbeing. The techniques surveyed are positive activity interventions, gratitude, humility, prosocial behaviour, compassion, maximising versus satisficing, experimental disclosure, the hedonic adaptation prevention model, bringing in the good and mindfulness. This is meant to be a thorough list of presently studied techniques, but there are doubtless some techniques that I have overlooked.

\section{Positive Activity Interventions}

Positive activity interventions (PAIs) are "simple, self-administered cognitive and behavioural strategies that can increase subjective well-being (happiness) by promoting 
positive feelings, positive thoughts and positive behaviours" (Shin and Lyubomirsky 2014). Examples of positive activities include writing letters of gratitude, counting one's blessings, practicing optimism, using one's strengths in a new way, affirming one's most important values ("experimental disclosure") and meditating on positive feelings towards oneself and others. These are all distinct from simply doing something pleasant, like eating some cake. The mechanism by which PAIs work to improve affect is theorised to be a combination of stimulating positive emotions and thoughts and satisfying basic psychological needs, specifically for autonomy and relatedness (Frederickson et al. 2008, Boehm et al. 2012). Many PAIs encourage pro-social behaviour and an awareness of others and their role in our lives, which encourages and reinforces feelings of relatedness. PAIs are also an active therapy, which provides patients with a sense of autonomous control over their psychological state. PAIs protect against negative affect by discouraging rumination and loneliness (Sin and Lyubomirsky 2009).

The effectiveness of PAIs is moderated by several factors. First, consistent application of PAI techniques over long time horizons is more effective than short courses of treatment. In the context of PAIs like gratitude and kindness that basically involve being a good person, this finding suggests that acting like a good person for a little while doesn't have the same effect on wellbeing as actually being a good person. Second, practising a variety of PAIs, varied randomly by the patient themselves, is more effective than mechanically practising the same PAI as instructed by a therapist. The implication here is that one must authentically feel gratitude, be optimistic etc. Finally, the activity must fit the person (Nelson and Lyubomirsky 2012). For example, collectivists may respond more powerfully to social PAIs like kindness than to internal PAIs like counting one's blessings.

\section{Gratitude}

The most well-known PAI is gratitude (McCullough et al 2002). In Western psychological science, gratitude is defined as "the recognition of a positive outcome from an external source, including a sense of wonder or thankfulness for the benefit received" (Nelson and Lyubomirsky 2016). Gratitude is distinguished from "appreciation" by the presence of an interpersonal component. That is to say, if you are grateful for the good things in your life or for something like a beautiful sunset, psychological science says you have "appreciation". Gratitude refers more specifically to thankfulness for positive things you receive from other 
people, especially in the form of altruistic acts that are privately harmful to the Samaritan (Bartlett and DeSteno 2006). Gratitude does not involve feelings of indebtedness, though it does encourage reciprocity.

Contemporary research distinguishes between gratitude as an emotional state that you enter when you recognise altruistic acts, and gratitude as a trait (Wood et al 2010). A person with a grateful disposition (trait) feels the emotion of gratitude more frequently and with greater intensity. More generally, grateful people orient themselves towards appreciating the positive in the world. Grateful people thus experience both gratefulness and appreciation on a regular basis. Interventions to boost gratitude include counting one's blessings (Emmons and McCullough 2003), keeping a gratitude journal where you write down the things you are grateful for, and simply encouraging people to notice when people do them favours and react by saying "thanks" (Emmons 2008).

The mechanisms through which gratitude affects well-being are unclear (Wood et al 2010). Indeed, it is still an open question whether wellbeing leads to gratitude or gratitude leads to wellbeing. However, experimental studies suggest gratitude interventions for wellbeing stimulate outcomes that no-intervention (typically wait-list) control groups don't receive. There is presently some empirical support for the following causal theories. First, focusing on positive rather than negative things inevitably increases the salience of positive feelings in one's conscious mind. This is particularly relevant for appreciation, which encourages individuals to actively enjoy the things they have rather than pine for what they don't or ruminate on their shortcomings. Second, gratitude instigates cognitive processes that see people reframe negative things in positive ways, such as "my layoff made me appreciate the support my family provides" (Nelson and Lyubomirsky 2016). Finally, gratitude dovetails with a range of prosocial behaviours and outcomes. Cultivating gratitude makes you more aware of the things others do for you, which opens your eyes to your social networks and encourages you to help them in turn, inspiring a virtuous cycle of mutual support. In general, gratitude deepens social relationships, satisfying our need for relatedness (Algoe 2012). The effects of gratitude on sociality are strong enough to have inspired an entire research effort dedicated to exploring the links between gratitude and avenues for the evolution of altruism and other pro-social behaviours in humans (McCullough et al 2008). 


\section{Humility}

A construct related to gratitude is humility. In psychological science, humility is defined by five observable indicators: a secure, accepting identity, freedom from distortion, openness to new information, other focus and egalitarian beliefs (Chancellor and Lyubomirsky 2013). This broad definition is strikingly divergent from tradition. Contrast the definition given by psychological science with the Oxford Dictionary: "humility: the quality of having a modest or low view of one's importance”. Synonyms include modesty, humbleness, meekness and unassertiveness. It is in the context of this definition that Nietzsche (1888/2000, Essay 3), Norton (1976) and all other philosophers who dislike the self-abnegation inherent in most religions attack humility as an unhealthy attribute. Yet in the definition used by modern psychological science, humility is transfigured from this arguably unhealthy, self-abnegating quality to being inextricable from the kind of self-awareness that is required for any sort of self-renovation.

The second half of the definition - other focus and egalitarian beliefs - is especially questionable. Not only does it have no historical basis, but it also seems to equate humility with a pinch of left-wing idealism. It is unsurprising in this context that humility scholarship struggled to find a coherent measurement strategy through most of the $20^{\text {th }}$ century (Davis et al 2010). Individuals on the left and right wing of politics have very different ways of understanding egalitarianism. As explained by Haidt (2012), the left wing emphasises relative equity - how far apart people are in terms of resources like wealth. The right wing instead emphasises proportional equity. This means getting what you deserve. Consequently, where the left-wing sees unemployment payments as critical for egalitarianism, the rightwing seems them as fundamentally inegalitarian, for example. In this context, unless survey questions measuring egalitarianism are phrased with great care, left wingers may score high on questions where right-wingers score low, and vice versa, not because of different degrees of concern regarding equality, but because of different intuitions regarding the meaning of the term. This makes the concept hard to measure and can easily bias results. Humility scholars insist that "a complete lack of a hallmark should disqualify one from possessing humility" (Chancellor and Lyubomirsky 2013). They suggest that anyone who "[approves] of inequalities among social groups in status, wealth and power" is not humble. Depending on survey design, this might precludes anyone with libertarian as opposed to Rawlsian values, to take a simple example, from having humility. 
Given this political and definitional baggage ${ }^{15}$, I will restrict my discussion here to empirical results pertaining to the first half of the new definition of humility: a secure, accepting identity, free from distortion and open to new information. I will use the phrase "welladjusted" to describe this collection of traits. Being well-adjusted is a prerequisite for many of the techniques described in this chapter, and certainly for the coalescence of being.

A secure, accepting identity is largely about ego-stability and consistent self-esteem. Volatility of self-esteem is associated with higher incidence and intensity of anger and hostility (Kermis 2005). Low self-esteem is associated with depression, delinquency and a tendency to externalise problems (Donellan et al 2005). How to bring about ego-stability and self-esteem is an ongoing project in psychology and the subject of the rest of this book, but some necessary conditions can be drawn from studies in humility.

First, ego-stability should not be confused with a self-image cast in iron that the individual never deviates from under stress from the external world. This goes against the eudemonic arguments of Norton (1976; see chapter 8). Instead, individuals' self-relevant beliefs should "express flexibility and abstractness" (Campbell et al. 1996). This is because abstract selfrelevant thoughts can shift with changing circumstances and information, whereas identities founded on specific goals or facts collapse easily as a result of failure to meet those goals. Empirical evidence suggests that the achievement of goals improves emotional wellbeing, but failure to achieve goals leads to sadness (Lyubormisky et al 2011). Of course, an identity based on abstract and flexible facts or goals is harder to precisely define, apply, be confident in and pursue, so there is a trade-off here. From a pragmatic point of view then, what is important is to be realistic in one's self-appraisal and open to changing one's mind in the face of new information. This mitigates the likelihood of failure and also allows for flexibility, without precluding the clear articulation of one's identity.

Second, one should have a compassionate regard for one's self-image. This means that you are honest about your deficiencies and weaknesses, including your ability to change them

\footnotetext{
${ }^{15}$ One other point that deserves a footnote is that these definitional issues might be fuelling empirical findings in a deceptive way. For example, there is a substantial body of work attesting to an upward spiral between gratitude and humility (Kruse et al 2014; Ruberton et al 2016). Yet this seems to be inevitable once you define humility as a fundamentally social virtue. There would be nothing wrong with this if researchers were examining the relationship between "other focus and egalitarian beliefs" and gratitude. The problem comes when they tie in being well-adjusted. At that point, the implication is that saying thanks and being more appreciative of your life gives you a more "secure, accepting identity, free from distortion and open to new information". While one could invent theory to justify this result, it seems much more reasonable to assume that the result is an artefact of the definitions the empirical studies start from.
} 
(Neff and Vonk 2009). There is a risk in this attitude that you become lazy about changing those aspects of yourself that you don't like. Such an outcome should be avoided. But equally, hating yourself for things that you cannot change is a toxic and counterproductive attitude. Compassionate self-regard is fundamentally about being reasonable with yourself. This is distinct from being soft on yourself, which simply perpetuates cognitive dissonance.

Finally, "freedom from distortion" is predominantly about being honest with yourself. Individuals free from distortion can claim responsibility for their mistakes and avoid taking credit for work done by others, and they do not exaggerate or denigrate their own achievements. An undistorted self-image is critical to the coalescence of being. Yet it is a difficult quality to cultivate. There is abundant empirical evidence for self-enhancement in people's self-perception, even among otherwise well-adjusted individuals. For example, most people see themselves as above average, even in the face of contrary evidence (Kruger and Dunning 1999).

\section{Prosocial behaviour}

Gratitude and humility both sit within a broader category of "prosocial" techniques for improving emotional wellbeing. There is some evidence that prosocial behaviour makes both the kind person and the recipient happy, and this happiness in turn encourages the recipient to reciprocate or otherwise "pay it forward" (Aknin et al 2012; Dunn et al 2008). One hypothesised channel for this is that prosocial behaviour and a prosocial environment enhance our sense of relatedness. Another proposed channel is purely chemical, where prosocial behaviours provoke a dopamine or other pleasant neurochemical response. This makes sense if prosocial behaviour is something we have evolved to improve our survival (Wilson 2015). Positive feelings associated with prosocial behaviour would encourage us to engage in such behaviours, thereby improving our fitness.

\section{Compassion}

A specific kind of prosocial behaviour that has received a measure of attention recently is “compassion", specifically as articulated in Buddhist philosophy. In recent work to define compassion so that it can be operationalised in empirical work, the following five 
characteristics were identified: 1) recognising suffering, 2) understanding the universality of suffering in human experience, 3) feeling empathy for the person suffering and connecting with the distress (emotional response) 4) tolerating uncomfortable feelings aroused in response to the suffering person and thereby remaining open to and accepting of the person suffering (non-judgement), 5) motivation to act/acting to alleviate the suffering (Strauss et al 2016). There are three orientations of compassion: compassion for others, receiving compassion from others and self-compassion (Jazaieri et al. 2013). There is a tendency among many people to avoid compassion because it seems to threaten self-interest or because compassion is considered a limited resource that should be reserved for one's immediate kin.

The benefits of compassion for others have already been discussed under pro-social behaviour, and the benefits of self-compassion were covered during the analysis of humility. What remains to be examined is receiving compassion from others. Some people may fear such compassion because they do not consider themselves worthy of it (Gilbert et al 2011). Others may actively reject it because it implies that there is something wrong with them. In this context, increasing an individual's capacity to receive compassion might make them more open to receiving help when needed, or pay more attention to the concerns of others about their present behaviour. Empirical work in compassion is in its infancy and thus, effects sizes for these benefits are not available.

An important point to make about compassion is that most contemporary psychological studies of it are tied to Buddhist philosophy and conducted by committed Buddhists. These authors underline that it is unclear whether the practice and benefits of compassion can be isolated or even exist independently of broader Buddhist practice and values (Dorjee 2014). Notably, compassion is a learned skill in Buddhism that helps the individual to practice loving kindness, notice and alleviate suffering and ultimately move towards enlightenment. It is thus intricately associated with Buddhist ethics and philosophy. In the absence of this ethical context, "compassion" becomes mostly about simply being a nice person.

\section{Maximising versus satisficing}

Studies of the behavioural tendencies of happy people have revealed that they tend to look for and quickly take "good enough" options when making decisions rather than investing time and resources to find the best possible option (Abbe et al 2003). This is the distinction 
between satisficers and maximisers. Several studies have demonstrated that maximising is negatively associated with happiness (Schartz et al 2002). This is not because maximisers make worse decisions. Indeed, the opposite seems to be the case. Instead, it is because they agonise over those decisions, even after they have been made. There is a cost in cognitive resources to this, not to mention an unnecessary burden of anxiety.

\section{Experimental disclosure}

Experimental disclosure involves writing and talking about life events as a form of therapy. In theory, disclosing the fact of such events and any feelings associated with them may allow people to free their mind of unwanted thoughts, help them to make sense of upsetting events, teach them to better regulate their emotions, habituate them to negative emotions and improve their connection with their social world. It's what has been happening on psychiatrist's couches for decades. A somewhat recent meta-analysis found statistically significant effects from experimental disclosure on emotional wellbeing, but the effect size was trivial, explaining only $0.56 \%$ of the variance (not half, but half of one per cent) in measured outcomes (Frattaroli 2006).

The issue might be that the ambit of experimental disclosure might be too broad. There is evidence that only writing and talking works for processing negative events, whereas simply thinking about positive ones has similar effects (Lyubomirsky et al. 2006). Writing and talking involve “organising, integrating and analysing one's problems with a focus on solution generation or at least acceptance". This processing can satisfy the desire to understand the meaning of an event, enhance understanding of its significance and create a narrative that links into the individual's identity (Singer 2004; Smyth et al. 2001). Processing takes the emotion out of events, which allows them to be reflected on without triggering distress. Obviously thinking is involved in both writing and talking, but their structured nature obviates against deleterious rumination in a way that thinking on one's own does not (Hixon and Swann 1993). Thinking rather than writing or talking about positive events is useful precisely because it limits processing and allows the individual to instead wallow in the positive emotional valence of pleasant past experiences (Lyubomirsky et al. 2006). 


\section{The hedonic adaptation prevention model}

This idea of retaining the emotional valence of positive experiences so that they can be replayed and reducing it for negative experiences so that they fade from consciousness is the basic idea in the hedonic adaptation prevention (HAP) model (Armenta et al 2014). The HAP was developed to counteract the natural tendency of individuals to adapt to positive experiences while accelerating adaptation to negative ones (Sheldon \& Lyubomirsky 2012). One of its principle insights is that experiences that generate a variety of positive thoughts and feelings have a more prolonged effect than those that generate only a single positive effect (Fritz et al 2017). For example, the effect of most material goods tends to fade because they provide only one sensation. By contrast, a new and enjoyable job can provide a plethora of positive things to reflect on, such as colleagues, location, the work itself, pay and a meaningful mission. In general, experiences have been found to produce longer lasting positive emotional valence than goods (Van Boven and Gilovich 2003).

Two other techniques frequently covered off in discussions of the HAP are appreciation and living in the moment. Appreciation in this context is the same as in gratitude-reflecting on what's good in life brings positive emotions to the fore, reducing cognitive space for negative ones. Living in the moment breaks down into four items: savouring, basking, marvelling and awe. When something good is happening to you, focus your attention on the positive feelings and do not think of the future. This is savouring, and it prevents the emotion from fading. If you can act on the experience, by say, throwing a party, that's even better, and referred to as capitalisation. Basking is the same technique as savouring applied when positive things are happening to other people that you have positive feelings for. Marvelling and awe are essentially savouring applied to things that are amazing or staggering, typically in nature, like a beautiful sunset or sublime athleticism (Bryant and Verof 2006).

It is worth noting that two behavioural tendencies that are in a sense the opposite of the HAP are rumination and leaning into adaptation. Rumination is "a mode of responding to distress that involves repetitively and passively focusing on symptoms of distress and on possible causes and consequences of these symptoms". Critically, "rumination does not lead to active problem solving to change circumstances surrounding these symptoms". People who ruminate remain "fixated on the problems and on their feelings about them without taking action" (Nolen-Hoeskema et al 2008). Unlike in the HAP on experimental disclosure, ruminators do not process their experiences, construct a healing narrative or look for 
solutions. They consequently build up the presence of negative emotions in their conscious experience rather than dissolving them. Leaning into adaptation involves a tendency to always focus on the future, the negative, the next step, or what remains to be maximised. Unsurprisingly, leaners adapt rapidly to improvements in their circumstances and consequently remain dissatisfied with life.

\section{Bringing in the Good and the HEAL method}

Another integrated strategy for improving affect balance similar to the HAP model is Hanson's (2013) notion of bringing in the good and the HEAL method he has developed for this practice. Bringing in the good is "the deliberate internalisation of positive experiences in implicit memory". Like the HAP, it is a suite of techniques that help individuals to focus on and thereby increase the intensity and duration of good feelings. Bringing in the good also emphasises the possibility of using positive experiences and to overwrite negative associations embedded in memory. For example, consider someone who suffers anxiety as a result of past bullying that occurred when they attempted to join a new social circle. This individual can focus on positive feelings associated with an enjoyable social experience in the present and use them to actively replace the association between social interaction, bullying and negative feelings they have in their mind. The HEAL method has four steps (Hanson, ibid. p. 61):

1. Have a positive experience: this involves either noticing a pleasant experience underway, such as a feeling of wonder, or creating one yourself by, for example, thinking about things you are grateful for.

2. Enrich it: stay with the feeling for a sustained period (10 seconds or longer) and try to bring it to the centre of consciousness.

3. Absorb it: Meditate on the feeling so that it occupies not just your consciousness but mind (and potentially body) more generally.

4. Link positive and negative material: bring negative associations into consciousness alongside your positive experience, thereby replacing those negative associations with the present positive ones. 


\section{Mindfulness}

Mindfulness is a concept in Buddhist philosophy and practice that Jon Kabat-Zinn adapted for use as a treatment for stress (Kabat-Zinn 2006). It has also recently been adapted as a treatment for preventing depressive relapse (Barnhofer et al. 2009). Kabat-Zinn's original definition of the term was: "paying attention in a particular way: on purpose, in the present moment, and non-judgementally". The definition employed in the Philadelphia mindfulness scale is "the tendency to be highly aware of one's internal and external experiences in the context of an accepting, non-judgemental stance towards those experiences" (Cardaciotto et al 2008).

These Western definitions of mindfulness are substantially more limited than the Buddhist one. Traditional definitions situate mindfulness within a broader sweet of activities that help the individual practice Buddhist ethics, let go of the self and attain enlightenment. The therapeutic effects of mindfulness derive from its ability to grant patients control over their conscious thoughts. In particular, mindfulness grants the ability to recognise emotions as not existing independently of mind. As we exercise some control over our minds, we can choose to let go of negative thoughts. This is referred to as renunciation (Ricard 2003). It can help individuals to avoid ruminative thoughts and control stress. Furthermore, the nonjudgemental aspect of mindfulness allows people afflicted with stress and depression to manage these emotions without them provoking feelings of self-hate or hopelessness (Keng et al 2011).

Some studies suggest that mindfulness can aid in values clarification and improved behavioural self-regulation (Roemer et al 2009; Shapiro et al. 2006). Attentional control is fundamental to self-awareness, which is in turn critical for self-actualisation. To understand who you are, you must be aware of your actions and decisions on a moment by moment basis. It is in the moment that your self-concept is disclosed. If you are unable to regulate your behaviour in line with your values, or if you are incapable of recognising when you are acting in- or out of line with your values, you cannot self-actualise. Non-judgement in the moment is important here, as judgement will cause you to expunge things in your conscious experience that you don't like and double down on those you do. This can be a hindrance to self-actualisation if you avoid confronting the fact that you are behaving out of line with your avowed self. Or it can be a boon when it allows you to regulate your subconsciously triggered behaviours so that they are in-line with your avowed self. You especially want to be non- 
judgemental in the discovery phase of self-actualisation when you are still trying to figure out who you are. In the affirmation stage, non-judgement of actions that are incongruent with your avowed self impedes self-actualisation.

This way of using mindfulness is somewhat antithetical to the way Buddhism applies the technique. In Buddhism, mindfulness allows the practitioner to:

...recognise the emotion at the very moment that it forms, understand that it is but a thought, devoid of intrinsic existence, and allow it to dissipate spontaneously so as to avoid the chain reaction it would normally unleash (Ricard 2003, p. 133).

Consistent application of mindfulness in Buddhism allows the user to gain control of the mind and through it, the substance of conscious experience. The end goal is to completely empty the consciousness of self:

If we want to be free of inner suffering once and for all, it is not enough to rid ourselves of the emotions themselves; we must eliminate our attachment to the ego. Is that possible? It is, because as we've seen, the ego exists merely as mental imputation. A concept can be dispelled, but only by the wisdom that perceives the ego is devoid of intrinsic existence (Ricard 2003, p. 130).

Where Buddhism seeks to dissolve the ego, the coalescence of being seeks to build, strengthen and multiply its ephemeral strands until it is no longer ephemeral, but deep, dense, precisely articulated, aware of itself and its nature over time and consistent in that nature. As we will see in later chapters, the purpose here is not to become free of inner suffering once and for all, but rather to become able to make sense of suffering and transfigure it into a source of meaning and joy in life (Nietzsche 1887). Furthermore, if you destroy the ego, there is nothing left to experience the good life. What remains is not happiness, but tranquillity (Schopenhauer 1844/1966). Mindfulness is a technique that can be used to bring about either the dissolution or consolidation of the self. This thesis argues for the latter in direct and explicit opposition to Buddhism.

\section{Conclusion}

This chapter demonstrated that it is correct, at least on the available evidence, to think of hedonia as a function of positive affect, negative affect, and hedonic life satisfaction. It 
further argued that hedonia is distinct from eudaimonia and despair. In particular, that hedonic satisfaction and existential satisfaction are distinct. In the next section, it discussed the importance of conceiving emotional wellbeing as a parameter that influences an individual's affective tendencies, in particular their disposition to feel particular emotions and be in particular moods. Emotional wellbeing is a function of eudaimonia and despair, and is one channel linking the three dimensions of wellbeing — hedonia, eudaimonia and despairtogether.

The second section of the chapter discussed some prominent characteristics of hedonia. The first was the peak-end rule and the curious tendency of people to remember hedonia differently to how they experience it. This lead to a discussion of two other prominent clusters of cognitive-behavioural theories associated with hedonia: reference points and the related phenomena of focusing illusions and loss aversion in wellbeing assessment, and adaptation by way of homeostasis and the hedonic treadmill. This section also discussed the literature in happiness economics, notably the impact of hope, desperation and inequality on investment decisions, declining marginal returns from income to wellbeing and the deleterious impact of unemployment.

Section three reviewed what we have learnt about hedonia from time use surveys. Perhaps the main takeaway point here is that pleasure and purpose are experienced differently and are often traded-off against one another. Work, for example, is good for purpose but not necessarily pleasure, and vice versa for watching television. We should be careful about overlooking either when thinking about wellbeing. Besides that point, the results are what you would expect: people don't like doing chores and commuting, and enjoy leisure and spending time with friends and family.

The final section of the chapter reviewed a range of techniques for mood management drawn largely from the cognitive-behavioural therapies literature. These assist the individual to achieve hedonia by encouraging constructive habits of behaviour and mind. In many cases they are formalisations of ancient wisdom (especially stoicism) and common sense, like being a good person, looking on the bright side and not sweating the small stuff. Other techniques are somewhat more complicated, like self-compassion and staying in the moment of positive events.

The techniques of mood management are important, but they have a somewhat shallow relationship to wellbeing because they rarely consider the deeper causes of emotions and 
mood in eudaimonia and despair. Emotion is a sophisticated signalling device. Fear, for example, sends our mind a message that we need to leave because we are in danger. In a similar way, positive and negative emotions accompany the process of self-actualisation. For example, a sense of achievement accompanies the completion of authentic goals, whereas only a sense of relief accompanies the completion of extrinsically-regulated goals. The coalescence of being uses these emotional signals as guides in the quest to overcome despair and achieve eudaimonia. As one progresses on that quest, the positive emotional signals one receives increase in frequency, duration and intensity, while negative emotions do the opposite. This is because you are engaging in fewer activities, hold fewer values and associate with fewer people who are 'wrong' for you. When you associate with the 'right' things, you get positive emotional signals, and you rarely get negative emotional signals because you rarely associate with the 'wrong' things. The key point to underline here is that while mood and emotion management is important, if one is in despair or not living eudaimonically then negative moods and emotions will be much more prevalent regardless of how competent you are at cognitive-behavioural therapy. As such it is necessary to approach wellbeing, including emotional wellbeing, holistically. We need to go beyond emotion and beyond mood management because Hedonia, eudaimonia and despair and interdependent. This task is taken up over the coming chapters. 


\section{Chapter 7}

\section{Eudaimonia}

It matters not how strait the gate,

How charged with punishments the scroll,

I am the master of my fate;

I am the captain of my soul

- William Ernest Henley, Invictus

\section{Introduction}

As with the previous chapter on hedonia, the primary purpose of this chapter is to justify the distinction of eudaimonia as a dimension of wellbeing and to explain the three variables it contains, namely autonomy, competence and relatedness. The chapter also explains the differences between eudaimonic ideas in psychology and philosophy, and introduces a range of ideas from self-determination theory (SDT) that are extremely helpful for understanding the process by which wellbeing is acquired.

The chapter proceeds as follows. I begin with the literature in psychology that emphasises the important theoretical and empirical differences between hedonic and eudaimonic conceptions of wellbeing. Early subjective well-being scholarship on the part of hedonic psychologists and happiness economists did not consider nor correctly discern empirically the central importance of relationships, autonomy, purpose and personal growth to wellbeing. Following the efforts of eudaimonic psychologists, these literatures are now steadily merging. Having established eudaimonia as a standalone dimension of wellbeing, I turn to explain the difference between philosophical and psychological conceptions of eudaimonia. The two literatures are unified by an emphasis on wellbeing as both a process and an outcome, and by their claim that the most prudent way to attain wellbeing derives from distinctive qualities of 
human nature. They diverge from there. Philosophical accounts are grounded in a view of humans as rational and ethical creatures and consequently emphasises the role of practical reason and virtue for attaining wellbeing. Psychological accounts instead emphasise that humans are organisms that have evolved to autonomously seek to expand their self, notably in terms of their skills and knowledge, and to integrate into groups that aid their survival. Psychological accounts consequently argue that the prudent way to pursue wellbeing involves nurturing basic psychological needs that emerge from this organismic basis.

The most influential of the psychological accounts of eudaimonic well-being is that of selfdetermination theory (SDT). It is to this body of work that the second half of the chapter is dedicated. I explain the nature of the three basic needs emphasised by SDT - autonomy, competence and relatedness - and review the empirical evidence that strongly suggests that nurturing these basic needs is fundamental to wellbeing. I then briefly review the evolutionary basis of SDT and the notion of humans being wired for organismic integration, which gives them their basic orientation towards growth and internal consistency. A critical aspect of this organismic perspective is the nature of motivation-why do we do some things and not others? The next section of the chapter explains SDT's theory of motivation and the important distinction between intrinsic and extrinsic motivation. The motivation spectrum and the process of internalisation, by which extrinsically motivated behaviours can gradually become intrinsically motivated, is a core idea in the coalescence of being. It is introduced in this chapter in detail. Finally, I review research from SDT on goal pursuit and why selfconcordant goals that are intrinsically motivated are strongly and positively associated with wellbeing while extrinsic aspirations are often negatively related to wellbeing. This research underscores that the prudential good must be understood both as process and outcome.

\section{Eudaimonia as distinct from Hedonia}

In the previous chapter on hedonia I reviewed arguments and evidence from people working within the subjective well-being tradition, including happiness economists and hedonic psychologists, which suggested that hedonia and eudaimonia are distinct aspects of wellbeing. Here I review additional arguments and evidence, this time from scholars working within the eudaimonic tradition. 
Arguably the first work in psychology claiming that affect and satisfaction were insufficient concepts to capture holistic wellbeing was Ryff (1989a, 1989b). She argued, as I did in chapters 1 and 2, that SW-B research had proceeded in the absence of theory and that this had caused that literature to miss important dimensions of wellbeing (1989b, p. 1069):

The premise of this study is that there has been particular neglect at the most fundamental level in...the task of defining the essential features of psychological well-being. It is argued that much of the prior literature is founded on conceptions of well-being that have little theoretical rationale and, as a consequence, neglect important aspects of positive functioning.

After reviewing theoretical work in the history of philosophical and psychological thought on wellbeing, Ryff posited a 6-factor model of wellbeing. It included the following: selfacceptance, positive relations with others, autonomy, environmental mastery, purpose in life and personal growth. She then conducted a statistical analysis, the results of which suggested that these dimensions were important aspects of wellbeing and that affect and life satisfaction were insufficient to capture them (Ryff 1989b, p. 1077, emphasis added):

Certain of these instruments showed convergence with prior indexes of wellbeing...However, other dimensions - most notably, positive relations with others, autonomy, purpose in life and personal growth-were not as closely tied to current assessment indexes, as evident in both bivariate and multivariate analyses. These findings support the claim that key aspects of positive psychological functioning emphasized in theory have not been represented in the empirical arena.

What are the implications for past and future research on wellbeing? Primarily, they suggest that the previous literature has been guided by somewhat narrow conceptions of positive functioning. Central emphasis has been given to shortterm affective well-being (i.e. happiness) at the expense of more enduring life challenges such as having a sense of purpose and direction, achieving satisfying relationships with others, and gaining a sense of self-realization...Similarly, life satisfaction, despite its more enduring, long-term quality, has failed to monitor such features of well-being as autonomy, personal growth and positive relations with others. 
Three of the dimensions emphasised by Ryff — relations, autonomy and personal growth — are central themes in SDT's account of positive human functioning. Similarly, autonomy, purpose and especially personal growth features in what came to be known as eudaimonic accounts of psychological well-being, notably in the work of Waterman (2013). He published a series of works (1990, 1992, 1993, 2007a, 2007b) developing the notion of eudaimonic wellbeing as grounded in personal expressiveness and providing statistical evidence that this aspect of wellbeing was poorly represented in SW-B research. A key finding of Waterman's work was that while experiences of eudaimonia are always accompanied by positive affect, the reverse is not true. This finding relates to the idea presented in the previous chapter regarding existential satisfaction. It is possible to be periodically happy and even generally satisfied with your life while still experiencing feelings of nausea, ennui and stagnation, all of which negatively impact wellbeing. Furthermore, it is not possible to address these deep determinants of ill-being simply by treating mood and affect. In contrast, someone who is triumphing over despair and nourishing their needs will almost certainly have positive affect and be satisfied with their life because affect and satisfaction guide the process by which despair is overcome and the needs nourished.

Waterman's efforts eventually culminated in a discussion between him and prominent researchers within SW-B in the Journal of Positive Psychology (Kashdan et al 2008, 2009, Waterman 2008). In their papers, the SWB scholars expressed reservations about eudaimonic wellbeing owing to difficulties operationalising it, the diversity of conceptions of eudaimonic wellbeing, confusion about what constituted wellbeing as an outcome as compared to things better conceptualised as causes of wellbeing or part of the process by which it is achieved, and the differences between eudaimonic philosophy and eudaimonic psychology. However, they ultimately concluded that wellbeing scholarship needed to pay more attention to the factors highlighted by eudaimonic scholars, including relationships, meaning, autonomy and personal growth. The eudaimonic perspective has grown in prominence in hedonic psychology and happiness economics since (OECD 2017, Stone and Mackie 2013, p. 5). Incidentally, this thesis is an attempt to provide the synthesis and clarity Kashdan et al (2008) are concerned is lacking in non-hedonic accounts of wellbeing.

Waterman was supported in his efforts by a handful of other scholars, especially Vitters $\varnothing$ and others associated with the "functional" approach to psychological well-being (Vitters $\varnothing 2009$, 2010, Vitters $\varnothing$ et al 2013, 2014) and researchers in SDT. Following an influential publication in the journal of happiness studies merging SDT and eudaimonic perspectives in psychology 
and philosophy (Ryan et al 2008), SDT has consistently been described by its leaders as a eudaimonic approach to wellbeing (Ryan and Deci 2017, p. 612).

\section{Eudaimonia in philosophy and psychology}

The term "eudaimonia" has a long history stretching back to Aristotle. Commensurate with these illustrious origins, eudaimonia has been the object of extensive study in philosophical circles and only more recently in psychology. Chapter 9 integrates some aspects of the philosophical accounts into the coalescence of being. This chapter focuses almost exclusively on the psychological accounts, notably the one emanating from self-determination theory. It is worthwhile in this context to briefly delineate the psychological account of eudaimonia from the philosophical account, and to consider overlaps and potential complementarities between the two.

In both philosophical and psychological accounts, eudaimonia concerns what it means to "live well". This notion of living wellbeing means that eudaimonia is about human flourishing in the sense of wellbeing-as-process more than wellbeing-as-outcome, though outcomes certainly feature, especially in psychological accounts (Besser-Jones 2015). Furthermore, eudaimonic accounts of wellbeing think that it must be explained in terms of the distinctive features of human beings. In this regard, Aristotle emphasised our capacity for reason and morality and so defined eudaimonia as activity of the soul in accordance with reason and virtue (Aristotle NE 1999, 1.7).

This emphasis on practical rationality as fundamental to wellbeing is inherent in most contemporary philosophical conceptions of eudaimonia (Annas 2011, Russell 2012, LeBar 2013) but absent from psychological accounts. Psychologists tend to be sceptical of emphasising reason because there is extensive empirical evidence that we don't behave in accordance with reason, and this is true even of people who have high levels of wellbeing and reasoning ability. We are influenced by unconscious biases (Bargh and Chartrand 1999, Kahneman 2011), and some of these are conjectured to have important buffering effects on self-esteem and other aspects of wellbeing (Johnson et al 1997). Many of our behaviours are unconscious and automatic (Doris 2002, Kahneman 2011). And our moral reasoning tends to come after moral instincts, which are what actually guide our behaviour (Haidt 2001, 2012). 
Rather than reason and virtue, psychological accounts instead ground their conception of eudaimonia in basic human needs, the kinds of lives that nourish these needs and the positive states that flow from living and satisfying the needs in this way (Ryan and Deci 2017, Ryff and Singer 2008). The psychological accounts argue that humans have evolved to be a particular kind of organism that is programmed to behave so as to satisfy these basic needs and that the organism will experience ill being if it deviates from this nature. As a result, psychological accounts of eudaimonia share with philosophical accounts the idea that eudaimonia is about living well, that the determinants of wellbeing are objective (in this case, whether basic psychological needs are met) and the idea that this nature of wellbeing emerges from the distinctive nature of humans.

\section{The basic psychological needs}

SDT posits three basic psychological needs: autonomy, relatedness and competence. A basic need is defined first and foremost by its direct causal relationship with optimal development, psychological integrity, health and wellbeing. This causal relationship goes in both directions: the frustration of needs leads to ill-being just as their nourishment leads to wellbeing (Ryan and Deci 2017, p. 251). As a logical consequence of this definition, basic psychological needs are posited as objective determinants of wellbeing. Someone who is not nourishing these needs will be unwell, regardless of how effective their mood management or the extent to which they are satisfying their preferences. Basic psychological needs have been empirically demonstrated to be universal features of human psychology that cut across cultures and genders (Ryan and Deci 2017, ch. 22).

Autonomy is the need to self-regulate one's experiences and actions. An autonomous individual feels volitional, congruent and integrated (De Charms 1968, Ryan 1993, Shapiro 1981). They are not externally controlled. The behaviours of an autonomous individual are self-endorsed and self-concordant in the sense that they align with the individual's authentic motivations and values. Autonomy in SDT is not about independence, self-reliance, freedom from all social influences, detachment from others, or individualism (Ryan and Deci 2017 p. 568). This is critical for understanding the relationship between autonomy and relatedness, and for responding to critiques from social relativists who argue that SDT is an excessively Western and individualistic doctrine that does not hold in collectivist cultures (Iyengar and Lepper 1999, Markus and Kitayama 2003). Succinctly, an individual can autonomously 
promote the needs of the group and autonomously comport towards other individuals. There is no conflict between autonomy and relatedness and nothing in self-determination theory that makes it incompatible with collectivist cultures. I will return to these matters in chapter 9.

Competence is about being good at the things you want to be good at. It refers to a need for mastery and effectiveness. It is implicated in a huge range of behaviours, from athletics and video games to scientific research and puzzle solving. However, it is also easily thwarted by excessive challenge, pervasive negative feedback and overwhelming social comparisons (such as to professional athletes).

Relatedness concerns having healthy and satisfying relationships with valued others. More generally, it is about social connectedness. It is nourished both by being cared for by others and by being valuable to others, notably as a result of contributions to the group. Relatedness is closely related with to a "sense of belonging" (Baumeister and Leary 1995) and a sense of being integral to social organisations — what Angyal (1941) called homonomy.

There is extensive cross-cultural empirical evidence to support the hypothesis that nourishing the basic psychological needs improves wellbeing in terms of positive affect, life satisfaction, ease of motivation, vitality, self-esteem, and the absence of psychopathology, depression, anxiety, compartmentalisation, defensiveness and personality rigidity (Chen et al 2015, Church et al 2013, Sheldon et al 2004, 2009). These results have been extended to specific domains including the workplace (Deci et al 2001, Ilardi et al 1993, Baard et al 2004) and schools (Jang et al 2009). Variation in the degree to which basic needs are nourished predicts differences in wellbeing between individuals, and variation in the degree to which each need is nourished predicts changes in wellbeing within individuals (Sheldon et al 1996, Reis et al 2000, La Guardia et al 2000, Lynch et al 2009).

\section{The evolutionary basis of self-determination theory}

Self-determination theory has contributed a range of other ideas to the wellbeing discourse that are important for understanding wellbeing holistically, especially with regards to the process by which wellbeing is attained. I will address three of these ideas in detail in the rest of this chapter: the evolutionary basis of SDT, the motivation spectrum, and the notion of self-concordant goals. 
Evolutionary perspectives have swept across the psychological sciences in recent decades. The basic principle of evolutionary psychology is that there is such a thing as "human nature", including in terms of psychological processes and features, and that this nature is the product of evolutionary forces (Barkow et al 1992). Evolutionary psychology has brought a kind of discipline to the psychological sciences by requiring that postulated aspects of human nature be able to be grounded in evolutionary processes. If some postulated quirk of human psychology can't be understood as an evolutionary adaptation then its veracity is immediately called into question.

SDT is grounded in a view of human nature that neatly addresses the evolutionary basis of the theory's basic tenets. SDT posits a process of organismic integration that is inherent to human beings (Ryan and Deci 2017, p. 29):

...individuals are thought to possess an inherent, active tendency toward the extension, progressive transformation, and integration of structures, functions, and experiences. By continuously stretching their capacities, expressing their propensities, and integrating new skills and knowledge into existing structures, people develop in the direction of greater effectiveness, organisation and relative unity in functioning. Regulation of action based on a synthesis of experiences and values provides the basis for a coherent and vital sense of self and integrity.

A succinct way of saying this is that humans have a tendency towards personal growth, but the concept runs deeper than that. In particular, the three basic psychological needs emerge directly from the idea of organismic integration. Humans have adapted to be inquisitive beings that seek to improve their competence as this is critical for survival - they must learn navigation, the crafting and use of tools, language, self-defence and a range of other skills to flourish. The psychological need for competence evolved around the acquisition of these skills. Humans are also aided in their survival by collective action and have consequently evolved a tendency towards social behaviour (Baumeister 2005, Greene 2013, Wilson 2015). This is the evolutionary basis of the need for relatedness. The need for autonomy emerges in parallel to intrinsic motivation as a basic rudder guiding behaviour towards adaptively advantageous growth and integration (Ruiz-Mirazo et al 2000, Santelices 1999). Environments and groups that thwart the basic needs undermine the tendency of humans towards organismic integration. This naturally gives rise to distress in the form of depression, 
anxiety and psychopathology as the psychophysiology of the human animal reacts to its odds of survival being diminished (Slavin and Kriegman 1992).

Organismic integration also undergirds the psychological tendency to avoid and overcome compartmentalisation. Integration requires different aspects of the self to be coherent. If different aspects of the self are inconsistent they will pull the individual in different directions, leading to goal conflict, undermining motivation and causing distress.

\section{The motivation spectrum}

SDT is foremost a theory of motivation: an account of why humans undertake certain actions and not others, and why they feel certain behaviours to be relatively effortless to engage in while others require willpower or even duress. The principle device for understanding motivation in SDT is the notion of a spectrum of motivation running from extrinsic to intrinsic, with more intrinsically motivated tasks being easier to undertake because they are more self-determined. In contrast, the more extrinsically motivated a behaviour the more selfregulation is required to engage in it. Extrinsically motivated activities are undertaken either to avoid extrinsic punishment or to attain rewards that are contingent on the activity, such as approval. Intrinsically motivated behaviours are those where the activity is its own reward, so to speak. Individuals can gradually bring behaviours and values from extrinsic motivation to intrinsic motivation through the process of internalisation, described below. Intrinsic motivation and the associated need for autonomy emerge out of the postulate of an organismic tendency towards integration. This tendency must be grounded in a primordial, subconscious inclination towards certain activities. Activities that align with this core inclination come naturally - they are intrinsically motivated.

The spectrum of motivation begins at one end with "controlled" behaviours, also known as extrinsic regulation. These are behaviours motivated by external agents, typically via duress. They cause distress to the controlled individual and are draining, reducing vitality.

Introjected behaviours are one step closer to intrinsic motivation. Like controlled behaviours they are motivated by factors contingent to those behaviours, but unlike in controlled behaviours that are motivated by external duress, these contingencies are administered by the individual themselves (Deci and Ryan 2000, p. 236). The prototypic examples are contingencies of self-worth like pride, shame and guilt. The latter two are related to moral 
opprobrium, which links them to contingencies of positive regard by others (Tangney and Tracy 2012). Another important and common example is activities undertaken for contingent love, especially from parents and peers.

Identified behaviours are another step closer to intrinsically motivated. Individuals do not engage in them for their own sake, but because they recognise their value. They thus require less intense self-regulation than introjected behaviours. A common example is exercise. While many people dislike jogging, especially in the early days when one is unfit, they jog because they consider health intrinsically valuable. This makes motivating oneself to jog easier, despite jogging being an introjected behaviour (health is a contingent value in this case). Because of the more palpable element of volition in these behaviours, they are meaningfully more autonomous than introjected behaviours. However, they are not engaged in for their own sake, so they are not intrinsic behaviours either.

Integration constitutes the point of transition between extrinsic and intrinsic motivation. At this stage, the positive valuations that underpin identified behaviours are integrated into the individual's broader value system. They thereby become part of the individual's core identity and thus become more intrinsically motivated. For example, as the individual becomes better at jogging and joins running groups they may start to enjoy running and more thoroughly assimilate it into their lifestyle. It moves from being something that is done simply for health to something that is done for its own sake.

A better example, if I might be permitted to draw on my own life experiences for illustration, is my pursuit of mathematical competence. Not so long ago, I detested mathematics.

However, I wanted to study graduate economics and for this I required mathematics. As such, I identified with mathematics - I came to value it — but pursuing it required substantial willpower and self-regulation. Over time, I discovered branches of mathematics that were very useful to research work that I was intrinsically motivated towards. Statistics and econometrics ${ }^{16}$, in particular, came more easily to me in terms of motivation than say, trigonometry, because I could see the applications of statistics in helping me to answer questions about what causes happiness. As I started to more deeply appreciate the usefulness of mathematics to my research it began to connect more thoroughly with these other aspects

\footnotetext{
${ }^{16}$ Econometrics is a branch of statistics pioneered by economists that focuses on causal identification rather than the size of correlations. It is far more influential in the social sciences than conventional statistics because social scientists do not have access to laboratory conditions that make causal identification straightforward.
} 
of my identity and thereby became integrated. I still don't have a lot of intrinsic motivation towards mathematics, but it requires much less willpower to engage in it now than when I first got started.

Internalisation sees behaviours move from identification through integration to intrinsic motivation. It occurs when identified behaviours nurture the basic psychological needs for autonomy, competence and relatedness. When these needs are not nurtured the identified behaviours tend to be aborted. For example, individuals will affiliate with identified groups so long as those groups provide them with support and other goods and accept them as a valued member of the collective. If this does not take place, the individual will experience ostracism and ultimately abandon the group barring unusual circumstances. Similarly, individuals will undertake valued activities unless they prove consistently incompetent at them. The classic case here is New Year's Resolutions. Finally, individuals will undertake identified behaviour provided this grants a sense of autonomy. It is notable in this circumstance that experimental evidence suggests that the provision of contingent rewards can actually undermine intrinsic motivation (see Deci et al 1999 for a meta-analysis).

\section{Intrinsic and extrinsic values and self-concordant goals}

A final insight pertaining to wellbeing emerging from research in SDT is that all goals are not created equal. Specifically, goals targeted because they are associated with extrinsic and contingent rewards of money, fame and image do not produce the same wellbeing benefits at attainment as goals associated with intrinsic rewards of personal growth and intimacy (Kasser and Ryan 1993, 1996). Indeed, attaining extrinsic goals can even be negatively associated with wellbeing (Kasser and Ryan 2001). Moreover, even the mere pursuit of so-called "extrinsic aspirations" can be actively harmful to wellbeing as the process involved in this pursuit does not nurture the basic psychological needs. In contrast, the process of achieving "intrinsic aspirations" does nurture the basic needs and so pursuing such goals is wellbeing enhancing regardless of whether the goals are actually attained (Howell et al 2011, Sheldon et al 2004, Sheldon and Krieger 2014). Experimental evidence suggests that this poor relationship between extrinsic aspirations and wellbeing holds even in social contexts, like business schools and corporate law firms, which espouse extrinsic aspirations like money and power and celebrate their achievement (Kasser and Ahuvia 2002, Vansteenkiste et al 2006). 
Sheldon has expanded these insights into a more general theory of goal self-concordance. The self-concordance model posits that people will derive greater wellbeing from goals that fit their personalities, or more daringly, their innate selves (Sheldon and Vansteenkiste 2005). Now innate qualities vary across persons and are very difficult to measures, but intrinsic motivation can act as a proxy in empirical work (Sheldon and Cooper 2008, Sheldon and Schuler 2011). Part of the power of the model comes from its relationship to selfactualization. It is by muddling through to self-concordant goals - directed by the negative affective signals that accompany the pursuit and achievement of disconcordant goal and the positive affective signals that accompany the pursuit and achievement of concordant goalsthat we can "bootstrap ourselves into our own futures, arriving at the future of our choice" (Sheldon 2013, p. 126). This is reminiscent of the Hellenic maxim, commonly repeated in eudaimonic texts, to "become who you are". I will return to the notion of self-concordance and its relationship to goal setting and achievement in chapter 9.

\section{Conclusion}

SDT's nuanced theory of goal pursuit and attainment is important because it underscores that there is a right and wrong way to go about wellbeing. The prudential good-what is "good for" someone-is a combination of prudential outcomes and prudential pursuit of those outcomes. Pursuing extrinsic aspirations is unlikely to be beneficial to wellbeing. Relatedly, SDT's findings concerning extrinsic aspirations also underscore that "preference satisfaction" is an insufficiently nuanced way to conceptualise wellbeing. The satisfaction of some preferences are unlikely to result in wellbeing. Preference-satisfaction accounts of wellbeing are often aware of such issues and deal with them through the notion of "laundered" preferences (Adler 2013). SDT's research on aspirations contributes to our understanding of what laundered means in reality and what the process of laundering involves.

More broadly, SDT's sophisticated combination of organismic principles, motivation and the notion of basic psychological needs bridges objectivist and subjectivist ways of conceiving wellbeing. SDT and eudaimonic accounts of wellbeing more generally are typically categorised as objectivist theories of wellbeing. This is understandable - the basic psychological needs are posited as objective indicators of wellbeing. However, the need for autonomy and the motivation spectrum make it clear that individuals approach these objective ends in thoroughly subjective ways. What goals, values and behaviours an 
individual is intrinsically motivated to pursue will be unique to that individual - they are entirely subjective. What specific competencies and relations nourish the individual's basic psychological needs will be substantially, indeed mostly, dictated by their subjective makeup and assessments.

In the next two chapters I expand on SDT's insights, integrating them with a range of ideas from continental philosophy and other theories in psychology. I will expand the subjectiveobjectivist foundation of SDT with critical insights about the role of affect — of hedonia — in guiding the process by which individuals discover their intrinsic self and navigate towards it. I will merge SDT's theory of internalisation with ideas from self-discrepancy theory to produce a simple mechanism that describes the central processes of self-actualization. I will bring in ideas from philosophy and social psychology pertaining to the nature of introspection and social verification to give a deeper account of what living in accordance with practical reason actually means. I will also return to the literature surveyed at the beginning of this chapter on the need for meaning and purpose, demonstrating that it is a prominent dimension of wellbeing and one that cannot be fully understood without also paying attention to issues of identity and virtue. 


\section{Chapter 8}

\section{Despair}

There is but one truly serious philosophical problem and that is suicide. Judging whether life is or is not worth living amounts to answering the fundamental question of philosophy.

- Albert Camus

\section{Introduction}

As with the respective chapters on hedonia and eudaimonia, the primary purpose of this chapter is to justify the inclusion of the despair dimension in the wellbeing production function, justify its three subcomponents — nausea, anguish and seriousness - and explain their nature. Despair was the motivating theme of existentialism, a prominent school of continental (European) philosophy that existed from around 1850 to 1950 . This period had to deal with the aftermath of the enlightenment's disenchantment of the world - what Nietzsche called "the death of God". The tremendous progress of reason during the enlightenment period had made it increasingly difficult to believe the teachings of Christianity, and Europeans were consequently grasping around, increasingly frantically, for stable sources of meaning, identity and palpable norms. The loosening force that the enlightenment exerted on European culture was exacerbated by the emergence of the modern metropolis, an environment that did not encourage the close knit, tight cultural communities that characterised agrarian life. Large cities also allowed people unprecedented free expression in the anonymity of crowds while making it difficult to really stand out. Drawing identity from the collective was thus made harder by cities while they simultaneously undermined the ability of people to be seen as unique individuals by others (Simmel 1903/1950). Anguish, nausea and seriousness were the inevitable, organic products of this milieu, and people's 
desperation to mitigate them contributed to the rise of fascism and totalitarianism. These ideologies provided thick norms based on race or class and enforced by the state. Similar forces are today contributing to the polarisation of politics and the rise of nationalist populism in the United States and Europe. Now is thus a timely moment to revisit the insights of existentialism, which have been almost entirely absent from the resurgent wellbeing discourse. While empirical research has recently caught up to the themes of existentialism, the full depth of that philosophical movement is not yet appreciated.

The chapter is set out as follows. First, I review Kierkegaard's notion of despair, which is arguably the first systematic articulation of existentialism's core problems. I then distinguish the three existential problems articulated by the French existentialists: nausea, seriousness and anguish. These three problems are contained within Kierkegaard's writings, but it is easier to work with them as three separate concepts rather than one unified but relatively clumsy idea. Succinctly, nausea refers to the sense of meaninglessness that characterizes the world after the death of God; seriousness refers to the feeling that ethics and values more generally lack significance and authority over the individual's conduct in the absence of a cosmic order to enforce them; and anguish refers to the inability of the individual to attain being or identity owing to the perpetual dialectic of the psyche. In part 2 of the chapter I review a raft of research in modern psychology attesting to the importance of nausea, seriousness and anguish for understanding wellbeing. This bolsters the case for including the existential problems in the wellbeing production function and also gives them greater texture and a more solid empirical footing. In the third part of the chapter I canvass solutions to despair that have been proposed in the philosophical literature. Specifically, I examine Kierkegaard's faith-based solution, Norton's eudaimonistic approach, and the roots of the coalescence of being in the work of Nietzsche and the French existentialists. These philosophical roots provide the skeleton upon which I foist theories and evidence from recent research in psychology to articulate the coalescence of being in the next chapter.

\section{The Existential Problems}

\section{Despair}

The problems and themes of existentialism were arguably first articulated in the modern era by the Danish philosopher Soren Kierkegaard, especially in his The Sickness unto Death 
(1849/2008). He uses a single term to encapsulate these problems: despair. From the outset, despair is characterised as concerning human "being” (Kierkegaard 1849/2008, p. 9):

Despair is a sickness of the spirit, of the self, and so can have three forms: being unconscious in despair of having a self (inauthentic despair), not wanting in despair to be oneself, and wanting in despair to be oneself.

To understand Kierkegaard's notion of despair, it is necessary to engage with his definition of the "self". This is given in very arcane terms (Kierkegaard 1849/2008, p. 9):

Spirit is the self. But what is the self? The self is a relation which relates to itself, or that in the relation which is its relating to itself. The self is not the relation but the relation's relating to itself. A human being is a synthesis of the infinite and the finite, of the temporal and the eternal. In short a synthesis. A synthesis is a relation between two terms. Looked at in this way a human being is not yet a self

To translate: "self" is reflexive consciousness, that is, self-awareness. "Spirit" in Kierkegaard's philosophy is thus "I". The reason why self is a "relation that relates to itself" is that consciousness can stand apart from the rest of a person. We can reflect on ourselves. Indeed, we seem to be able to change ourselves through conscious effort.

The final part of the definition, which refers to human being as a synthesis of the temporal and the eternal and thus "not yet a self", is the most important. The "temporal and the eternal" refers to the fact that individual humans grasp that they are both the same person over time - Usain Bolt, for example — and also that they are a different person at each point in time-Usain Bolt before and after breaking the 100m sprint world record, for example, or before and after retirement. The reason why we are "not yet a self" is that we are always becoming. We as individuals are defined by the choices we make over time. Consciousness and free will mean that these choices are never pre-determined. As such, who we are in the next moment is always an open question and we are always engaged in an ongoing process of self-actualisation. The existentialists would later summarise the relationship between consciousness and becoming in one of their two slogans: "existence precedes essence". The idea here is that we are born a human being, but it is the nature of human beings to be constantly constructing an individual identity through their unique choices: "man first of all 
exists, encounters himself, surges up in the world - and defines himself afterwards" (Sartre and De Beauvoir 1946, p. 3). ${ }^{17}$

Consciousness is a powerful thing. As the French existentialists would later emphasise, it makes us ontologically free-we are at liberty to define ourselves, in particular our values. Consciousness means that we are not automatons: slaves to our genetic programming and instinctive reactions to external stimuli. Kierkegaard (1849/2008, p. 9) emphasises these boons:

The possibility of this sickness is man's advantage over the beast, and it is an advantage which characterises him quite otherwise than the upright posture, for it bespeaks the infinite erectness of loftiness of his being spirit.

Yet while consciousness is powerful and liberating, it also brings with it the sickness of despair. Existentialism as a school of philosophy would come to distinguish three subcomponents of despair: anguish, seriousness and nausea.

\section{Anguish}

Anguish emerges first. As a conscious being, we are always cut-off from ourselves in the moment of decision (Sartre 1943/2005, p. 62). As such, we lack an identity that we can hold fixed over time - we cannot definitively answer the question "who am I?" because we are always becoming. Furthermore, we are capable of agonizing over decisions. This is particularly true in the early stages of self-development because immature identities often contain conflicting values, rationales, desires and identities. They are segregated. As such, our "self" cannot provide clear guidance about what we should do in certain circumstances. We will be conflicted and paralysed with doubt in those circumstances. Anguish takes in these two issues: the absence of a fixed identity and the ability to agonise over decisions when the self is not unified (Sartre 1943/2005, p. 53):

If our analysis has not led us astray, there ought to exist for the human being, in so far as he is conscious of being, a certain mode of standing opposite his past and his future, as being both this past and this future and as not being them. We

\footnotetext{
${ }^{17}$ See also Nietzsche (1886/2000, p. 250): "[man is] the as yet undetermined animal”.
} 
shall be able to furnish an immediate reply to this question; it is in anguish that man gets the consciousness of his freedom, or if you prefer, anguish is the mode of being of freedom as consciousness of being; it is in anguish that freedom is, in its being, in question for itself.

Kierkegaard's three forms of despair all concern anguish. The first form-being unconscious in despair of having a self-refers to those individuals who are not aware that they are moral agents and masters of their own destiny. They do not suffer from anguish, but only because they do not comprehend the significance of their choices. The second form, not wanting in despair to be oneself, refers to people who flee the responsibility associated with being a moral agent — what the existentialists would later refer to as "bad faith". Eichmann who was "just following orders" as he sent thousands of Jews to their deaths is the archetypal example (Arendt 1963/2003). The final form-wanting in despair to be oneself-refers to individuals who want to define themselves and use integrity to guide their decision making but cannot because consciousness means that we are always becoming, inescapably.

\section{Seriousness}

The third form of despair brings two other subcomponents of despair into the picture alongside anguish: seriousness and nausea. As mentioned, seriousness is the feeling that our values lack authority. In later existentialist philosophy, notably the writings of the French existentialists, the primary force undermining seriousness is the death of God. Without a cosmic order to undergird and enforce morality, moral rules come to appear arbitrary and in any case are easily ignored as they do not attract retribution from cosmic justice (Sartre and De Beauvoir 1946, p. 6):

The existentialist, on the contrary, finds it extremely embarrassing that God does not exist, for there disappears with Him all possibility of finding values in an intelligible heaven. There can no longer be any good a priori, since there is no infinite and perfect consciousness to think it. It is nowhere written that 'the good' exists, that one must be honest or not lie, since we are now upon the plane where there are only men. Dostoevsky once wrote that if God does not exist, then everything is permitted; and that, for existentialism, is the starting point. 
Kierkegaard is writing before the death of God as declared by Nietzsche (1887/1974, p. 181) and is a deeply religious Christian. As such, the chief force undermining seriousness in his philosophy is not the absence of God, but an absence of faith. Without belief in a cosmic order, the individual is necessarily confronted by the capriciousness of norms (1849/2008 $\mathrm{p}$. 83):

It recognizes no power over itself; therefore, in the final instance it lacks seriousness and can only conjure forth an appearance of seriousness, even when it bestows upon its experiments its greatest possible attention.

This is because an individual in the third form of despair is responsible not only for creating their own values but also for enforcing them upon their own behavior. Consciousness means that this enforcement is never absolute, because we are always capable of contradicting ourselves. Our values thus appear whimsical, "made-up" and superficial. Kierkegaard makes this point eloquently in the following passage (1849/2008 p. 84):

The self is its own master, absolutely (as one says) its own master; and exactly this is the despair, but also what it regards as its pleasure and joy. But it is easy on closer examination to see that this absolute ruler is a king without a country; that really he rules over nothing; his position, his kingdom, his sovereignty, are subject to the dialectic that rebellion is legitimate at any moment.

Sartre makes a similar point when he underlines that past pledges on the part of conscious being exert little binding force on decisions in the present (Sartre 1945/2005, p. 57):

...but what he apprehends then in anguish is precisely the total inefficacy of the past resolution. It is there doubtless but fixed, ineffectual, surpassed by the very fact that I am conscious of it. The resolution is still me to the extent that I realize constantly my identity with myself across the temporal flux, but it is no longer $m e$ - due to the fact that it has become an object for my consciousness. I am not subject to it; it fails in the mission which I have given it. 
Only we can hold ourselves to our promises because consciousness means that we can always decline to follow through. What this means is that we are always capable of contravening our ethical prerogatives, even those we previously avowed ourselves. God's cosmic order was a way around this issue because it promises eternal suffering to any who go against the rules. But if values are subjective and if the cosmic order does not exist, then the only immediate consequences of contravening your values is you being disappointed in yourself. Indeed, there may be no consequences, because you may decide that your past values were erroneous. This ease with which we can generate, assert and then contravene values speaks to their lack of seriousness. If we are the only authority over our values and conduct, then our values exert little binding power.

A critical corollary point to draw out here is that in existentialist philosophy (exempting Kierkegaard's owing to his theism) values are subjective. Values are not written into the firmament. In the words of Greene (2014), they "do not exist out there on some Platonic shelf" waiting to be discovered by someone with sufficient capacity for moral reasoning. Reasons can be given to justify certain values, but the extent to which these reasons are experienced as binding over individuals is dictated by how compelling those individuals feel those reasons to be, not by some cosmic order of justice. This is the link that connects anguish and seriousness most directly. We are the source of values but we also cannot ever justify these values by reference to something other than ourselves (Sartre 1943/2005, p. 62):

...it follows that my freedom is the unique foundation of values and that nothing, absolutely nothing, justifies me in adopting this or that particular value, this or that particular scale of values. As a being by whom values exist, I am unjustifiable. My freedom is anguished at being the foundation of values while itself without foundation.

\section{Nausea}

Nausea is closely related to seriousness. Nausea is the feeling of meaninglessness (literary explorations are available in Camus 1942/2013 and Sartre 1938/2000). In the absence of any cosmic order to imbue the world with transcendental purpose, the world comes to merely be, it does not matter (Reginster 2006). Meaning and purpose are closely related to value. When 
something is valuable, striving for it becomes somewhat meaningful. If values are made-up and capricious, then it is hard to sustain a sense of purpose when pursuing them, especially in the absence of pleasure or other crude rewards. A cosmic order not only supplies values-it also makes things transcendentally valuable. The goods of a cosmic order are valuable across time and space. Playing your part in God's plan, for example, contributes to the grand design of the universe, and secures you an eternal place in paradise. Without faith in a cosmic order, the best an individual can hope for is to pursue a life that is meaningful to them for the duration of their lives. Perhaps the bleakest articulation of this sentiment comes from De Beauvoir in the following passage, where she seems to invoke the inevitable heat death of the universe (De Beauvoir 1947/2002, p. 120):

The future stops too; beyond there is nothing more because nothing more is disclosed. From that formless night we can draw no justification for our acts, it condemns them with the same indifference; wiping out today's errors and defeats, it will also wipe out its triumphs; it can be chaos or death as well as paradise: perhaps men will one day return to barbarism, perhaps one day the earth will no longer be anything but an icy planet. In this perspective all moments are lost in the indistinctness of nothingness.

Nausea was a major theme of Nietzsche's philosophy. He argued (1887/1974, p. 75) that it was a fundamental part of the human condition:

Gradually, man has become a fantastic animal that has to fulfil one more condition of existence than any other animal: man has to believe, to know, from time to time, why he exists; his species cannot flourish without a periodic trust in life_-without faith in reason in life.

These words would sound familiar to anyone who has ever examined the literature on "the meaning of life". Nietzsche argued that the most pervasive and powerful source of meaning to date was religion, in particular the ascetic ideal. Religion imbues the universe with meaning - the cosmic order. The meaning of life becomes about comporting oneself to God's plan, or nirvana, or whatever else a religion dictates. This meaning is external-it lies outside the individual. This is why Nietzsche argued that "Men of faith... are necessarily dependent people, the sort of people who cannot posit themselves as a goal, who are utterly incapable of 
positing goals out of themselves" (Nietzsche 1885/1990, p. 54). He counselled the opposite approach: embrace the meaningless of existence as a joyous liberty:

Nobody is responsible for people existing in the first place, or for the state or circumstances or environment they are in. The fatality of human existence cannot be extricated from the fatality of everything that was and will be. People are not the products of some special design, will, or purpose, they do not represent an attempt to achieve an 'ideal of humanity', 'ideal of happiness' or 'ideal of morality'-it is absurd to want to devolve human existence onto some purpose or another. We have invented the concept of 'purpose': there are no purposes in reality ... a person is necessary, a person is a piece of fate, a person belongs to the whole, a person only is in the context of the whole - there is nothing that can judge, measure, compare, or condemn our being, because that would mean judging, measuring, comparing and condemning the whole ... but there is nothing outside the whole! The fact that nobody is held responsible any more, that being is not the sort of thing that can be traced back to causa prima, that the world is not unified as either a sensorium or a 'spirit', only this can constitute the great liberation - only this begins to restore the innocence of becoming...the concept of God has been the biggest objection to existence so far ... we reject God, we reject the responsibility in God: this is how we begin to redeem the world. (Nietzsche 1889/1990, p. 182)

Nietzsche was the first of the existentialists to appreciate that the existential problems all emerge from ambiguity - from the fact that we want to know with certainty who we are, what is right, and what we should do. When the universe does not provide these answers we become anguished and nauseated. The typical response to this ambiguity, as terrormanagement theory demonstrates empirically (see below) is to search around in one's cultural environment for sources of identity, meaning and seriousness. The existential problems emerge with greatest force in ages characterised by the breakdown of such cultural environments, with the early metropolitan period in Europe and deindustrialised communities in present day America being prime examples. Existentialism argued that this quest for certainty was either doomed to failure or to be engulfed in totalitarianism (fascist or socialist). The correct response is to embrace ambiguity, but this is a bitter pill to swallow. I will discuss their solution to despair in the final part of this chapter. Before that I will review 
more recent empirical work in psychology that attests to the veracity of the existentialist's claims.

\section{Scientific evidence for the importance of the existentialist perspective}

In this section I want to review cross-disciplinary literature and empirical evidence that attests to the importance of the themes of existentialism for understanding wellbeing. This serves two purposes. First, it strengthens the case for including the despair cluster in the wellbeing production function. It shows that multiple lines of inquiry have converged on nausea, anguish and seriousness as central aspects of the wellbeing. It also provides some solid empirical support for the claims of the existentialists, as many of the literatures I will be referring to are quantitative and experimental in nature. Second, it makes existentialist themes more accessible. Existentialism, like all continental philosophy, is written in at times impenetrable language that turns off a lot of parties who would otherwise be interested in its insights. The literatures to which I refer below are typically written in a more straightforward manner. Furthermore, as these literatures span multiple disciplines and are often interdisciplinary in nature, demonstrating their connection to existentialist thinking should reveal that existentialism is not a niche field but actually part of a broader landscape of inquiry into nausea, seriousness and anguish.

I begin with the literature on nausea or, as it is more commonly referred to, "meaning and purpose". This is because of all the themes of existentialism, it is nausea that has received the most attention in other literatures, including recently in SW-B scholarship (OECD 2017, Diener et al 2010, Seligman 2011). I move from there to seriousness, which is studied as part of contemporary inquiries into culture, norms and community disintegration, including as part of the literature on eudaimonic wellbeing. The final literature I examine is that coming out of terror-management theory (TMT), which argues that humans have a primordial aversion to death and uncertainty. They respond to this terror by comporting themselves towards meaning making communities. TMT segues nicely into the issue of anguish, which is fundamentally about the ambiguity of norms and existence. Anguish is inadvertently a feature of any inquiry into identity and personal narratives. 


\section{Nausea}

The literature arguably most engaged with the role of meaning in well-being is research in the logotherapy tradition in clinical psychology. Logotherapy was pioneered by Victor Frankl, most famously in his short work Man's Search for Meaning (1946/2008; see also Frankl 1969, 1975, 1978, 2000, 2010). It emphasises, echoing Nietzsche, that human's need meaning, that the absence of meaning can cause depression and other psychological ills, and that meaning-based therapies can help people suffering from a range of mental illnesses, particularly those associated with "existential vacuum". Logotherapy has been championed in America in particular by Victor Wong (see Wong 2012). Several studies in this vein speak to the relationship between meaning and wellbeing (see Steger 2010 for a review). A relationship has been found empirically between positive affect and meaning (Keyes et al 2002, King et al 2006, Steger et al 2006). Similarly, empirical studies have found a correlation between meaning in life and life satisfaction (Bonebright et al 2000, Ryff 1989, Steger 2006, Steger and Frazier 2005, Steger et al 2008). More broadly, meaning has been linked to a range of traits including autonomy, self-control and sense of control (Steger et al 2008, Garfield 1973, Newcomb and Harlow 1986, Reker and Peacock 1981), ego-resiliency (Tryon and Radzin 1972, Shek 1992) and positive perceptions of the world (Simon et al 1998).

There are other literatures concerning meaning and wellbeing. Religion is empirically associated with higher levels of subjective well-being (Ellison 1991, Witter et al 1985, Lim \& Putnam 2010, Dolan et al 2008). Scholars of religion have argued that a key driver of this association is religion's capacity to give people a sense transcendental meaning (Emmons 1999, Emmons et al 1998, Steger 2005). This echoes both Kierkegaard's argument that faith is the way out of despair (see below) and the argument of Nietzsche and the French existentialists that nausea emerges most powerfully after the death of God.

Baumeister has written about the role of meaning in wellbeing at book length (1991). He subsumes into the notion of meaning many of the themes that I separate out into eudaimonia, anguish and seriousness. However, he also draws important links between meaning and man's nature as "the cultural animal" (see Baumeister 2005 for a longer treatment). A critical advantage that homo sapiens possesses over other animals (and perhaps even over other members of the homo genus - see Harari 2011) is that we are capable of symbolic language. 
That is, we are capable of agreeing to shared meanings that we project through culture and its attendant tropes, notably art and ritual. This capacity is wired into us and has allowed our species to survive and flourish. Given this evolutionary background, it is reasonable to suspect that our neurochemistry is inclined to make us feel good when we interact with meaning and encourages us to seek out, create and interact with meaning.

With all this empirical work being done, one might ask why we need existentialist philosophy at all to help us appreciate the importance of meaning to wellbeing. The reason why existentialism needs to be more thoroughly integrated into this scholarship is because it is more theoretically deep than any other literatures, or at least, it brings novel insights. In particular, because other literatures did not typically emerge as a direct response to atheistic nihilism, they sometimes proceed as though the acquisition of meaning is a relatively straightforward affair. They advise people to "go out and do something meaningful" or "reinterpret your suffering as meaningful", as though this is easy. A principal contribution of existentialism is to recognise that in a nihilistic universe populated by loose, secular cultures (see below) meaning is very hard to achieve. Existentialism provides the basic building blocks of the process - namely the coalescence of being — by which meaning can be wrestled from such a hostile universe. Similar things can be said for its contribution to our understanding of seriousness and anguish.

\section{Seriousness}

The last twenty-odd years have seen tremendous progress in the study of the evolutionary psychology of moral cognition and sociobiology more broadly. A motivating question of much of this research is: how can altruism exist? The driving force of evolution is the selfish gene and its competition with other selfish genes. Such a gene is understandably altruistic towards kin because kin share a lot of genetic material. But why would it care about entities that aren't kin, including other members of its species? What gives rise to cooperation at the level of the group? This question is particularly stark in humans, where a group can number millions of members and where such groups can form along seemingly arbitrary lines like hobbies and nationalities (a social construct if ever there was one). An additional complexity in humans is that we are so clearly selfish to a substantial degree. We are not ants or bees- 
we do not operate as if by some sort of hive mind — nor are we a herd, and our groups are much larger than packs.

Breakthroughs have come in the last few decades out of the idea that natural selection can take place at both the level of the individual and the level of the group (Wilson 2015). While selfish individuals typically outcompete altruistic individuals, altruistic groups outcompete selfish groups. It is thus potentially fitness enhancing to be able to cooperate and behave altruistically, and we should not be surprised to observe cooperative groups and species flourish in the long run. ${ }^{18}$

Morality is part of the elaborate psychic and cultural architecture that we evolved as a species to help us cooperate in ever larger groups. The evolutionary chain is very simplistically the following. We first developed shared intentionality (Tomasello et al. 2005). This is the ability to grasp what someone else wants to do, which is necessary for any cooperation. Shared intentionality allows for behaviours like one person pulling down a branch while another picks the fruit. Both parties then share the meal. The next evolutionary step came after we started to form ever larger groups to achieve ever more lucrative collective tasks, like hunting mammoths and sacking villages. We developed group-mindedness, which is the cognitive architecture required for culture (ibid.). In the words of Jonathan Haidt, it grants us "the ability to learn and conform to social norms, feel and share group-related emotions and ultimately, to create and obey social institutions, including religion” (Haidt 2011).

The way this cognitive architecture manifests in moral judgement is superbly explored in Joshua Greene's Moral Tribes (2013), particularly his analysis of results from what is jokingly called trolleyology. This is the study, through laboratory experiments, of the decision-making processes involved in the now infamous trolley problem, an ethical hypothetical where there is a runaway train about to hit 5 people. You, a bystander, can hit a switch that will divert the train onto a different track where it only kills one person. Most people hit the switch. In brain scanners, their frontal lobe - the part of the brain responsible

\footnotetext{
${ }^{18}$ A note here for the philosophers: altruism in this context should be understood as 'other regarding' rather than in the pure sense of "privately harmful behaviour". An individual cooperating for the sake of their own survival is obviously still selfish - that is in a sense the whole point of this new way of understanding individual versus group selection. A selfish individual who chooses to cooperate does so because on balance, it is the best course of action for themselves. This is not necessarily in any "rational" sense, but rather in a psychic sense. Selfdetermination theory's notion of self-regulation provides the psychological tools for understanding how a selfish individual could nonetheless engage in altruistic behaviour, and even come to consider such behaviour selfdetermined and nourishing. There is always an element of the self that chooses to do what is good for the group, and that element does it for the self, not for the group.
} 
for conscious processing (as opposed to instinct) is typically very active. The great mystery of trolleyology was that when people were instead given the chance to push a fat man off a bridge in order to stop the train the majority of people thought this was impermissible. In the brain scans, their brain stem and amygdala — elements of our brains carried over from our animal ancestors - were more active than their frontal lobe. Why did people change their decision given that the outcome is the same in both cases: one dead instead of five?

The answer lies in the different regions of the brain. The frontal lobe is responsible for the cool calculation of conscious processing. It is not emotional, and tends towards utilitarianism. The parts of the brain that light up in the bridge case, however, are responsible for instinctive decision making. For some reason, in the primordial era of our species, we had good reason to develop a cognitive module that instinctively prevented us from lashing out at our peers. That's quite easy to explain once you're across the altruism literature: it's hard to get a reputation as a trustworthy co-operator if you have a tendency to smack your peers, or in this case, literally throw them under a bus. Conscious processing came later for a range of reasons, one of which is its ability to help us cooperate in very large groups, as evinced by its ability to justify a utilitarian decision to kill someone to save five others.

This distinction between quick, intuitive, system 1 moral instinct and slow, thoughtful, rational system 2 moral theorising is a major theme of Jonathan's Haidt The Righteous Mind (2011). He explores it therein using the metaphor of an elephant and its rider. The elephant is system 1-our ancient moral instincts. The rider is system 2-our much more recently evolved rational faculties. Moral instincts are depicted as an elephant rather than a horse because elephants are so much larger and smarter than horses. When the elephant moves, the rider can do little to control it, even if a moral instinct seems irrational. Indeed, Haidt presents research that suggests it is often more apt to think of the rider as serving the elephant, and not the other way around. Notably, instinctive moral feelings come first, and we then use our frontal lobe to rationalise these feelings. As Haidt (ibid, p. 38) explains;

What the rationalists were really doing was generating clever justifications for moral intuitions that were best explained by evolution. Do people believe in human rights because such rights actually exist, like mathematical truths, sitting on a cosmic shelf next to the Pythagorean theorem just waiting to be discovered by Platonic reasoners? Or do people feel revulsion and sympathy when they read 
accounts of torture, and then invent a story about universal rights to help justify their feelings?

A powerful piece of evidence for this comes from work on moral dumbfounding. This is a common phenomenon where people exert sustained effort to try to rationalise a moral instinct but even when they cannot do so, they still don't change their mind. In one example, people are presented a hypothetical case where a brother and sister commit incest while using extensive birth control, then agree that it was lovely and satisfying for both of them but they won't do it again. A large number of respondents condemn their behaviour, but then cannot rationalise their condemnation. They first hunt for some sort of victim. When they can't find one, they say things like "it's just not done you know...". But they rarely change their minds and condone the behaviour. Another piece of evidence comes from studies employing hypnosis. Some participants are hypnotised to feel disgust when they hear a benign codeword. Out of hypnosis, they are then told a story that includes their code-word about, for example, some guy named Simon who puts in a lot of volunteer work to run a school theatre production. Triggered respondents concoct fantastical reasons why Simon is actually a terrible person, like that he is a popularity seeking snob (Haidt, ibid. p. 62). Control-group respondents display no such prejudices. Haidt catalogues a range of other studies using different methodologies that replicate these results.

The key lesson, Haidt argues, is that "judgement and justification are separate processes" (ibid. p. 50). Furthermore, 'We do moral reasoning not to reconstruct the actual reasons why we ourselves came to a judgement; we reason to find the best possible reasons why somebody else ought to join us in our judgement' (ibid. p. 52). The point to underline is that morality is an evolution that is tied to social opprobrium and approval. This explains why people tend to behave more ethically in laboratory experiments where they believe they are being watched (Burnham 2003, Burnham and Hare 2007, Ernest-Jones et al 2011, Haley and Fessler 2005, Nettle et al 2012). This parallels the arguments of Lerner and Tetlock (2003, p. 433), who write:

A central function of thought is making sure that one acts in ways that can be persuasively justified or excused to others. Indeed, the process of considering the justifiability of one's choices may be so prevalent that decision makers not only search for convincing reasons to make a choice when they must explain that 
choice to others, they search for reasons to convince themselves that they have made the "right" choice.

It is unsurprising in this context that the moral emotions of shame, guilt and self-esteem are tied to moral trespass. These emotions and their physical cues, like blushing with embarrassment, help us to track social signals, show contrition and, ultimately, to learn, obey, operate in and act to change normative codes within normative communities. Leary (2012) has argued that self-esteem is a kind of sociometer that provides you with an affective gauge of how attractive you are as a partner or comrade. This perspective explains the omnipresence of gossip in our society—social judgement requires obsessive commentary on people's reputations. It also explains why God, that moral adjudicator par excellence, needs to be allseeing (Wilson, 2002).

The take-away point from all this discussion of evolution, culture and moral cognition is the following, from Greene (ibid. p. 329):

Morality is not what generations of philosophers and theologians have thought it to be. Morality is not a set of freestanding abstract truths that we can somehow access with our limited human minds. Moral psychology is not something that occasionally intrudes into the abstract realm of moral philosophy. Moral philosophy is a manifestation of moral psychology. Moral philosophies are, once again, just the intellectual tips of much bigger and deeper psychological and biological icebergs. Once you've understood this, your whole view of morality changes. Figure and ground reverse, and you see competing moral philosophies not just as points in an abstract philosophical space but as the predictable products of our dual-process brains.

Or more succinctly: "Morality is a set of psychological adaptations that allow otherwise selfish individuals to reap the benefits of cooperation" (ibid. p. 23). Haidt's (ibid, p. 314) definition of a moral system makes a similar point:

Moral systems are interlocking sets of values, virtues, norms, practices, identities, institutions, technologies, and evolved psychological mechanisms that work together to suppress or regulate self-interest and make cooperative societies possible. 
So morality is not objective (and by extension, neither is value). It is instead a function of our current social and environmental circumstances and helps us to prosper in those circumstances. Little wonder then that our morals have evolved so much since the renaissance as society has changed rapidly. Little wonder too, that different cultural groups existing in different environments have developed different moral codes. Now if value is subjective then we run straight into the problem of seriousness as articulated by the existentialists. Analytical philosophy has not yet caught up to their insight into these matters. This is perhaps because the Anglophone countries are only now having the existential crisis that hit Europe in the $20^{\text {th }}$ century. America remains a very religious nation, for example. As the America's existential crisis bites deeper, the negative impacts of a lack of seriousness on SWB will become more apparent.

\section{Anguish}

There is a relatively small body of empirical work that connects the evolutionary psychology of moral cognition with the themes of existential philosophy by way of the human aversion to ambiguity and our need for seriousness. This is the literature from terror management theory (TMT), which grew out of a desire to experimentally engage with postulates in existential philosophy and anthropology (Burke et al 2010). The foundational idea of TMT is that consciousness makes humans unique among animals in that they can foresee their own death. This provokes in humans a profound, primordial terror. We have evolved a range of defence mechanisms to prevent this terror from becoming debilitating, the most prominent of which is culture, which explains the world and imbues it with meaning and value (Greenberg et al 2004, p. 16). The march of science in modernity has undermined our ability to buy into many of these cultural systems, notably religion. As Nietzsche foreshadowed, the death of God opened an existential vacuum. Nationalism and materialism sufficed to plug this gap for some time in the $20^{\text {th }}$ century, but we are now in existential freefall, hence the rise of partisanship, the explosive growth in self-help books and the precipitous rise in mental health incidence.

Experimental results in TMT are typically derived using what is called a mortality salience condition. Participants are randomised into a control and a treatment group, and the treatment group is manipulated in such a way that death thoughts become more prominent in their mind either consciously, unconsciously, or both. Tests are then administered to determine what effect mortality salience has on different variables. Results indicate that people in mortality 
salience conditions have a higher need for closure than controls, suggesting discomfort with ambiguity or, in other words, discomfort with anguish (Dechesne et al 2000).

Two defences to mortality salience have been identified in existing research. The first is proximal defence, which is observable immediately following the salience manipulation but then fades quite rapidly. These include avoiding rooms with mirrors or writing short stories about oneself. Mirrors and reflective stories are behavioural options inserted into experiments by researchers that are theorised to get at the approach or avoidance of self-focus. Another common proximal defence is suppression, with experimental evidence showing that mortality salience often doesn't reach the level of consciousness, but increases at the subliminal level after manipulation (Florian and Mikulincer 2004, p. 61). Proximal defences demonstrate that humans have psychological systems designed to inhibit death thoughts.

The second defence mechanism is distal defence, which has a delayed onset. The principle form of this defence is an exaggerated regard (disdain) for similar (dissimilar) others. For example, after a mortality salience condition, participants were more aggressive towards other participants with divergent political views (measured by how much hot sauce they administered to them $)^{19}$ than were individuals in a control condition. Distal defences indicate a connection between social identity and the management of death-related thoughts.

Results in terror management research are mirrored by results in identity consolidation theory (IC), which focuses on people's responses to personal uncertainty, which is more specific than general ambiguity. Personal uncertainty is also distinct from focal uncertainty. Focal uncertainty refers to situations where the individual doesn't know how to express themselves, such as not knowing the most appropriate way to dress and act during a college orientation week (Sorrentino and Roney 2000, Sorrentino et al 2009). Such situations provide an opportunity for autonomous self-determination, and can end with one feeling that one is "being oneself". In contrast, personal uncertainty refers to "a kind of identity crisis that arises from awareness of conflict or lack of clarity about self elements" (McGregor 2004, p. 183). In such cases, there is no clear identity to guide behaviour, and multiple selves can offer conflicting advice, leading to dissonance, debilitation and potentially multiple approach-avoidance conflicts. This is extremely similar to the notion of anguish in existentialist philosophy.

\footnotetext{
${ }^{19}$ This is a pretty standard approach to measuring aggressiveness in psychological studies. It is a meaningful form of pain, but not physically severe enough to trigger obstruction by an ethics committee.
} 
IC posits four overarching strategies that people use to cope with personal uncertainty: integration, self-worth myopia, group identification and conviction. These strategies have parallels with those observed in terror-management. They are rewarding because they bring about self-regulatory efficiency, but they can also lead to narcissism, intergroup bias and zealous extremism (McGregor 2004).

The integration strategy for overcoming personal uncertainty involves building some life narrative and fitting new situations into that narrative. Such stories can make a life meaningful (McAdams 1993; see Nehamas 1985 for an existentialist account). Coherent stories also make information of fundamental importance to their narratives more accessible and psychologically consequential (McGregor and Holmes 1999). Experimental results indicate that people confronted with existential dilemmas react by planning activities and projects that are higher in integrity than control participants (McGregor et al 2001). Undertaking of such activities reduces personal uncertainty.

Self-worth is actually two techniques for managing personal uncertainty. The first is defensively enhancing one's self image when confronted by destabilising information (Tesser 2000). Individuals reminded of their self worth immediately after personal uncertainty priming are less likely to engage this defensive technique (Steele et al 1993). The second technique is self-worth myopia, which involves reducing the elements of one's self-concept that are salient at any one time to make inconsistencies across multiple selves less obvious and thus less troubling.

Group identification involves borrowing values from a group, which reduces the need for unpleasant self-analysis in times of personal uncertainty and buttresses identity in times of crisis. Similarly to terror management research, experimental evidence in IC suggests that personal uncertainty encourages intergroup bias (McGregor et al 2009, McGregor 2010, Van Den Bos 2009a, 2009b).

The final strategy is conviction and extremism. The basic idea here is to double down on one's present beliefs when confronted by threats to personal certainty. Experimental evidence is a little messy in this area. Individuals high in self-esteem demonstrate greater conviction in their beliefs when confronted with personal uncertainty than controls or people low in self-esteem (McGregor \& Marigold 2003). However, it is unclear whether this is because their views are more fully developed, in which case it is 
appropriate that they be more convinced of them, or if the conviction is unwarranted and merely acts to buttress self-esteem.

These results in terror-management theory and identity consolidation theory underline the importance of seriousness and anguish to wellbeing. People are distressed by ambiguity. They respond by doubling down on existing sources of meaning and seriousness, in particular group norms. They are similarly distressed by inconsistent identities and respond to this with deeper compartmentalisation or attempts to resolve inconsistencies.

There is an additional, important idea in TMT related to this last point about compartmentalisation or personal growth. This is that culture, by which is meant collectively held and reinforced value systems, can only effectively manage despair at the level of the individual if that culture is intrinsically chosen and integrated into the self by that individual. In cases where a culture does not fit the individual, dissonance will prevent the cultural worldview from managing despair, provoking the individual to reassess that worldview until they have something that does work. In this process, it is possible for an individual to reach a point where they have an acceptable cultural worldview but it is still weak in terms of its internal consistency. At this point, individuals may be inclined to engage in further values exploration, but this threatens the integrity of their cultural worldview and provokes existential anxiety. This tension between anxiety on the one hand and dissonance on the other can lead to developmental arrest and moral amplification of the type demonstrated by extremists (Haidt \& Algoe 2004; McGregor 2003). As Pyszczynski et al. (2012, p. 389) explain:

Ironically then, a secure worldview and sense of self-worth allows us to venture forth to uncharted mental territories where discoveries can emerge that question those very security-providing structures, requiring us to revise those structures to accommodate our self expansions... Unfortunately, we often fail to allow this dialectic process to continue its forward momentum; rather, we give up the potential pleasures of intrinsically motivated growth-promoting activity in exchange for the comfort and security that clinging to existing forms of psychological organisation of self and world provides.

So personal growth is enjoyable and worldview instability is unpleasant, but a measure of instability is required for growth. It follows that if an individual were able to develop an 
internal consistent worldview with which they were not dissonant while also fostering a degree of comfort with existential ambiguity (i.e. instability) they could frequently engage in personal growth without provoking too much disturbingly uncomfortable anxiety.

This hypothesis is confirmed by three clusters of empirical evidence. The first is that when people are primed to be creative in mortality salience conditions they engage fewer defensive mechanisms and are more comfortable engaging with existential questions (Routledge and Arndt 2009). The second is that individuals who go through near death experiences find themselves much more comfortable with mortality and consequently tend to move to affirm personal values rather than group ones and engage in more growth-inducing activities (Martin et al 2004). The third is that individuals with a low need for closure are more comfortable reflecting on issues raised by mortality salience manipulations than individuals with a high need for closure, who tend to increase belief in bogus but relevant theories, like horoscopes, when doing so reduces mortality salience (Dechesne et al 2000, 2003).

As we shall see, the coalescence of being is precisely an existential stance characterised by frequent personal growth that provoke little anxiety. It achieves this by providing a paradigm through which to practice personal growth that is grounded in an acceptance of the fundamental ambiguity of existence.

A final empirical literature worth discussing briefly in relation to anguish is the research on identity development. There is a large body of work exploring the importance of developing a clear sense of self through late childhood, adolescence and early adulthood (Luyckx et al 2006). This process can simultaneously involve depression, anxiety, openness and curiosity, with failure prolonging depressive and anxious episodes (Luyckx et al 2008). Themes from the literature on adolescent psychology are present in the more adult-focused literature on narrative therapy. This practice involves assisting patients to develop life stories and integrate traumatic or distressing events into identity through narratives that makes sense of them and provide meaning and closure (McAdams and McLean 2013, Angus and McLeod 2004). There is a proliferating body of literature in clinical psychology and contemporary explorations of wellbeing in public policy contexts that explores the role of ethnic identity in wellbeing and personal narratives (Crocetti 2008, Stronge et al 2016, Muriwai et al 2005, Yap and Yu 2016). All of this speaks to the importance of identity to wellbeing and the challenge that anguish can pose. 


\section{Philosophical solutions to the existential problems}

In the rest of this chapter I canvass some of the philosophical solutions to anguish, seriousness and nausea as articulated in existentialist philosophy. I analyse three solutions: faith in Kierkegaard, eudaimonism in Norton and the basic principles of the coalescence of being in Nietzsche and the existentialists. This analysis serves two purposes. First, it clarifies the three existential problems further. Second, it illuminates what a practical theory of wellbeing needs to be able to do to overcome despair and thereby make holistic wellbeing possible. This provides a launch pad for the practical theory elucidated in this work, namely the coalescence of being. I describe coalescence in much greater detail in the next chapter, drawing principally on literature from empirical psychology rather than philosophy.

\section{Faith}

Kierkegaard takes the existence of a cosmic order for granted, and sees despair as arising out of either an ignorance of this fact or an inability or refusal to believe in it. I emphasise "believe" because Kierkegaard accepts that God and the cosmic order is never verifiablefaith is fundamentally "in the absurd": faith is not rational. He argues that it is the "strength of the absurd" that allows faith to overcome despair. He is quite disparaging towards efforts to ground religiosity in reason (Kierkegaard 1843/2005, p. 40):

\footnotetext{
Would it not be best all the same to stop with faith, and is it not disturbing that everyone wants to go further? ... Would it not be better to remain standing at faith, and for the one who stands there to take care not to fall? For the movement of faith must be made continuously on the strength of the absurd, though in such a way, be it noted, that one does not lose finitude but gains it all of a piece.
}

Faith works to address the existential problems by arresting the psychic dialectic out of which despair arises. The self is then held fixed by God (Kierkegaard 1849/2008, p. 164):

The formula for that state in which there is no despair at all: in relating itself to itself and in waiting to be itself, the self is grounded transparently in the power which established it. Which formula in turn, as has frequently been remarked, is the definition of faith. 
An individual who has faith in the Holy Scriptures receives transcendental values from outside their self, and these values then determine their behavior in an authoritative way. Religious values are written into the firmament and this gives them a transcendental character. Moral laws are clearly defined in scripture and enforced by cosmic justice, which provides serious ethics. The individual has a place in God's plan, and by fulfilling their ordained role they can contribute to God's holy and beneficent goals. The apprehension of such a transcendental purpose overcomes nausea. Religion's serious, transcendental normative order overcomes anguish because the appropriate decision for an individual to make in any given situation is proscribed in the ethical commandments of scripture and the individual's role in God's plan. They need never anguish in the moment of decision, merely consult The Word. The veracity of this scripture is given additional symbolic authority by the strong cultural forces that typically underpin religious communities, including elaborate and ever-present rituals and iconography that make norms easily identifiable and interpretable, and in-group reciprocity and tight enforcement of social norms that reinforces notion of cosmic justice for believers and heathens (Wilson 2002, Gelfand 2011).

The "knight of faith" also need never anguish over the question "who am I?" because they receive the answer in contemplation of the lord. For Kierkegaard's knight of faith, the internal psychic dynamics of personality development are supplanted by the individual's comportment towards an external identity that they receive from an eternal God. Through prayer, communion and consultation with priests, the individual comes to sense God's intentions for them, which provides the "identity" that they are supposed to live. As this identity is part of the grand cosmic plan, it is given all at once, which arrests anguish, and transcendental, which provides meaning and seriousness. In this way, despair is arrested (Kierkegaard 1843/2005, p. 45). An example of an individual who appears to have behaved in the manner of the Knight of Faith is Martin Luther, one of the founders of the Protestant faith. He famously remarked: "I cannot and will not recant anything, for to go against conscience is neither right nor safe. Here I stand; I can do no other; so help me God. Amen”. 


\section{The crisis of faith}

While Kierkegaard is correct to the extent that faith can manage despair and dampen its effects, he is incorrect to suggest that it annihilates despair. The movement of faith begins with an individual introspecting, engaging with religious texts and communing with priests in order to discover their ordained place in God's plan. Once this is discovered, despair is annihilated so long as the individual maintains their faith that they have correctly identified their role in God's plan and that God and His cosmic order in fact exist. Faith in both of these items has to be maintained on the strength of the absurd because neither is a testable hypothesis. The possibility of a crisis of faith in this context seems substantial. The march of science since the enlightenment has called so much of revelation into questions that sustaining belief in the rest of the Bible, Koran or other religious texts is increasingly difficult. Conviction regarding one's own role is also ever open to the problem of the satanic verses: how is one to know whether it was God who sent you a message, and not Satan? Finally, the knight of faith seems to be just as open to the assaults of unexpected events as any other individual. By way of a simple example, consider a religious mother in the Bible belt of the United States. Following scripture, she regards homosexuality as a sin, and the care of her offspring as a duty. She then discovers that her daughter is a homosexual. She can only salvage her identity from this crisis by reinterpreting some aspect of her faith. This instance of inner normative conflict is an archetypal example of anguish. In this sense, faith is no different to the coalescence of being. Both involve positing values and affirming them. However, faith requires the reason for these values to be grounded in the absurd, whereas the coalescence of being accepts from the outset no ultimate justification for values other than individual's adhering to those values through integrity out of a desire to attain wellbeing. Coalescence does not rely on faith; it relies on subjectively compelling arguments.

\section{Eudaimonism}

How can a secularist solve the existential problems? Eudaimonism is one approach. Norton (1976) provides arguably the most thorough account of how eudaimonism works to address the existential problems. His is the only account of eudaimonism that I am aware of that specifically engages at length with existentialist philosophy. As such, I will focus my analysis on his account rather than less extreme theories of eudaimonia that are not formulated with 
reference to existentialism. More classical eudaimonism in the style of Annas (2004) is founded on the notion of objective goods. The evolutionary psychology literature that I surveyed above runs counter to this possibility. Norton's eudaimonism does not have this requirement.

Where faith requires the individual to comport towards an external standard of behavior embedded in scripture, Norton's eudaimonism advocates for an entirely inward-looking approach. The central tenet of eudaimonism is that each individual has an innate "true self"their daimon (Norton 1976, p. 14). "Eudaimonia" is the positive feeling that attends living in accordance with this true self (ibid. p. 5). It can be interpreted as the feeling that attends the absence of despair, and is often translated as "happiness", but happiness in the wellbeing sense rather than mere positive emotion.

It should be noted that Norton's eudaimonism is strictly about living in accordance with one's true individual nature. I emphasise individual because some readings of eudaimonism take it as involving merely the fulfilment of our human natures (Haybron 2008, p. 174). This is incorrect, at least insofar as Norton's eudaimonism is concerned. According to Norton, it is the nature of human's to have a daimon, and living in accordance with it brings wellbeing. But each individual daimon is unique, and so "nature-fulfilment" is a fundamentally subjective enterprise; lumping it in with objective-list theories of wellbeing like the capabilities approach of Sen and Nussbaum is misleading. The most important quote from Norton in this regard is the following (Norton 1976, pp. 9-10):

Our consideration of "personal truth" reveals that the great enemy of integrity is not falsehood as such but-ironically — the attractiveness of foreign truths, truths that belong to others... When an individual allows himself to be deflected from his own true course, he fails in that first responsibility from which all other genuine responsibilities follow, and whose fulfilment is the precondition of the least fulfilment of other responsibilities...Philosophically, this is formulated as the principle of ultimate varieties of value.

How does eudaimonism overcome anguish and nausea and secure seriousness? The power of eudaimonism in this regard comes from the fact that an individual's daimon constitutes a complete identity. It contains an individual's past, present and future. Only their present can 
exist at any one time (this is their "actuality"), but the daimon also contains all their potentiality, which the individual must actualise if they are to "become who they are" (Norton 1976, p. 229):

As we have noted previously, the mature lifetime of the integral individual is a single act, spread over time by the condition of existence that a thing cannot present itself all at once. But in a profound sense, integrity hereby abolishes time by containing its past and its future in its present... The past and future of the eudaimonic individual are contained in his present in the mode of that moral necessity conferred upon his life by his chosen principle of personhood.

The individual "discovers" their true self through introspection around the end of adolescence, and then lives their life in accordance with this true self. Certainly not all of their true self is present at once, but their commitment to living in accordance with their daimon (integrity) means that their values and behaviors are determined once and for all from the moment they orient themselves towards their daimon (Norton 1976, p. 223):

Eudaimonia's "wholeheartedness" means that the whole person is present in each of his acts. This is so because the individual's choice of his ultimate possibility establishes a principle of entailment whereby his future and his past are implicit in his present, and thereby are within his present act. Because his "there" is within his "here", he is devoid of that condition of semi-distraction that is the common attendant of personal life.

The "condition of semi-distraction" described in the quote above is anguish. Eudaimonism resolves anguish by providing the individual with a deterministic identity from the beginning of adulthood. Their decisions are guided by their adherence to the daimon they seek to actualise, annulling anguish in the moment, and their daimon is a richly conceived identity, annulling any anguish pertaining to the question: "who am I?"

Eudaimonism brings about seriousness through the combination of two factors. The first is the moral imperative of living in accordance with the daimon. Norton argues that the daimon represents each individual's personal perfection, and as such, they are morally obliged to actualise it (Norton 1976, p. 141): 
The metaphysics of self-actualization discloses that every "is" is also an "ought to be" in virtue of its promissory character, its foreshadowing of its own perfection. Affirmation of the actual world by actual beings is at the same time their responsibility to the perfection implicit in that actuality. Moral responsibility is inescapable because affirmation is inescapable.

This commitment to perfection provides the content of an individual's values, but it does not grant them seriousness; that comes from the necessity of integrity. Norton emphasizes that eudaimonism requires an individual to act with fealty to their daimon at all times (ibid. p. 193):

The actualization of personhood is progressive, requiring, in Nietzsche's words "long obedience in the same direction". To re-choose is to annihilate all accomplished actualization stemming from original choice; it is a re-beginning out of a lapse into indeterminacy. As such it poses to the life in question the specter of final indeterminacy as a life without identity or necessity — a life that in the true meaning of the term has failed to exist.

In Norton's philosophy, the individual has both an ethical and selfish interest in affirming their daimon. If they transgress its character they will be plunged back into: "a life without identity or necessity". The positive feeling of eudaimonia will dissipate, and they will be left unwell. So they must act with integrity. They must show fidelity to the identity (the daimon) that they have chosen to actualise over the life course. This injects seriousness into ethics by making the individual an effective authority for enforcing their own values upon themselves. The individual will resist those aspects of her psyche that suggest transgressing the daimon and will stick to the plan that is the daimon.

It is somewhat unclear how Norton's eudaimonism overcomes nausea, but I take it to be largely a matter of intrinsic motivation. The daimon is fundamentally internal and comporting towards it is accompanied by the positive feeling and state of eudaimonia. As such, it is reasonable to expect that the individual will be intrinsically motivated to live in accordance with their daimon, and this motivation will be reinforced by positive affective signals. Contemporaneous theories in psychology, notably Maslow's hierarchy of needs (Norton's 
book is dedicated to Maslow), suggested that this kind of self-actualisation is sufficient for achieving a sense of meaning. This view is sustained in more modern schools like selfdetermination theory (Ryan \& Deci 2000).

\section{The shortcomings of eudaimonism}

There are several flaws in the above arguments pertaining to eudaimonism's ability to overcome despair. The first is the notion that "perfection" is sufficient to ground ethics. This error runs all the way back to Plato. Perfection has a normative but not an ethical quality. It registers in the aesthetic sense, not the moral sense. For example, we can recognize that Floyd Mayweather is close to a perfect boxer. But while we may value this instrumentally, we do not give Mayweather moral credit for it. We do not think Mayweather is a "good" person because he is a "good" boxer. Indeed, Floyd Mayweather is widely regarded as a terrible person due to his history of domestic violence, which has nothing to do with perfection. I am here using a definition of perfection grounded in instrumentality, but the point stands if you interpret perfection to refer instead to mere actualisation of one's daimon, which may not have much instrumental quality whatsoever.

The research in the evolutionary psychology of moral cognition canvassed earlier suggests that ethical impulses evolved to help us cooperate in groups. These studies suggest that utilitarian and deontological considerations, particularly regarding issues of fairness and reciprocity, are fundamental to our moral cognitions. Norton's eudaimonism and its ethics of personal perfection can't engage with any of this, which severely hamstrings the extent to which it can provide serious (or indeed useful and practical) ethics.

The second flaw in eudaimonism is that the idea that you can discover your "true-self" in totality at the end of adolescence simply does not jibe with present theories in developmental psychology. ${ }^{20}$ It is reasonable enough to claim the existence of an innate self. Empirical evidence suggests a substantial role for genetic determinism of identity through a range of channels (Pinker 2002). Notably, our physical and intellectual characteristics and aptitudes

\footnotetext{
${ }^{20}$ Norton should be given credit for trying to ground his theory in the work of contemporary developmental psychologists, notably Piaget, but the science has moved on since.
} 
exert a significant determinism over the paths we can take in life. You cannot, for example, be a professional basketballer if you are short. Personality in general seems substantially hereditary, as do tastes and talents (Polderman et al 2015). However, there is a long way to go from this innate self to an actual self that constitutes an identity that is sophisticated, refined and deep enough to withstand anguish.

Developmental psychology nowadays emphasises genetic predispositions rather than predeterminants (Carver 2012). Metaphorically, we are born a lump of clay with certain dimensions, notably weight, but our final form is shaped through individual interaction with environmental factors. An elegant summary is provided by Neuroscientist Gary Marcus (2004, pp. 30-40): "nature provides a first draft, which experience then revises ... 'built-in' does not mean unmalleable; it means 'organised in advance of experience"'. Developmental psychology also emphasizes the existence of "multiple selves" that we must harmonise as our personality develops (Showers and Zeigler-Hill 2012). Adolescence is punctuated by a growing awareness of these multiple selves and "a dramatic rise in the detection of contradictory self-attributes that lead to conflict and confusion" (Harter 2012). This is a radically different picture to that developed by Norton. He posits that adolescents find themselves misunderstood by others and subsequently embark on a quest of self-discovery (Norton 1976, p. 111). Present developmental psychology instead argues that adolescents can't understand themselves and consequently set out on a quest of individuation that is part self-discovery through introspection and part self-creation through the affirmation of desired character traits (Higgins 1991). This process of identity formation is ongoing throughout life, so Norton's notion that we can choose an identity at the end of adolescence is questionable. Eudaimonism's ability to overcome anguish is thus limited.

The discussion in the paragraph above points to a third approach to overcoming despair that sits between faith and eudaimonism. I call the coalescence of being, or coalescence for short. It combines introspection - the internal - and environmental, especially social, engagement the external. It also involves both self-discovery and self-creation. I turn now to briefly pick up its origins in the work of Nietzsche and the French existentialists. I elaborate it in full with reference to the modern psychology literature in the next chapter. 


\title{
The coalescence of being in philosophy
}

The most fundamental tenet of coalescence is comfort with ambiguity: as Nietzsche (1886/2000, p. 343) said, "above all, we should not want to rid the world of its rich ambiguity". As discussed, there is a desire in humans for fixed meaning: a grand cosmic plan, serious values. But these things don't exist. We also want objective truth, but this is inaccessible. So there is no hope of certainty. ${ }^{21}$ As long as we look for certainty, we will suffer. So step one is to embrace the lack of certainty that characterises our existence (Nietzsche 1887/1974, sec. 346):
We have become cold, hard, and tough in the realisation that the way of this world is anything but divine; even by human standards it is not rational, merciful or just. We know it well, the world in which we live is ungodly, immoral, inhuman.

This is liberating. If objective normative truth exists then it exerts a binding power. We could not help but comport to it. Yet this would undermine our ability to be our authentic selves. Accepting that the universe is devoid of value and that objective truth is inaccessible gives us latitude to define our own values and affirm them in the world without inherent normative opprobrium. It is by relinquishing the need for certainty and instead embracing our capacity to will meaning and value that we overcome despair (Nietzsche $1887 / 1974$, p. 289):

\begin{abstract}
One could conceive of such a pleasure and power of self-determination, such a freedom of the will that the spirit would take leave of all faith and every wish for certainty, being practised in maintaining itself on insubstantial ropes and possibilities and dancing ever near abysses. Such a spirit would be the free spirit par excellence.
\end{abstract}

\footnotetext{
${ }^{21}$ Note that when I speak here of the inaccessibility of truth I am referring to the problem of induction, rather than the notion, common in postmodern circles, that objective truth does not exist. There is an objective world, and we can utter true statements about it. For example, we, a $17^{\text {th }}$ century European, might say that "all swans are white". We base this claim on the thousands of times we have observed swans and the fact that they were always white. We then travel to Australia and discover that some swans are black. There is no social construction here. Our original claim is refuted. We believed that all swans were white because we had no observed the entire population of swans. This is the problem of induction: we can never know whether truth claims we make on the basis of observation are simply a function of too small a sample. Pure mathematics does not fare any better, because mathematics begins either with axioms that are not drawn from reality or empirical facts that suffer from the problem of induction. The "way out" of this problem is to focus on falsification. If we subject a claim, like "all swans are white", to a battery of tough tests and we are unable to falsify it then we can treat it as a fact (not truth) until falsifying evidence comes along. This is the scientific method (Popper 1934). I am not denying objective truth, I am denying that we can access it.
} 
Note that ambiguity is distinct from absurdity. Ambiguity holds that the meaning of the world is not given but must be created through affirmation. Absurdity is the view that the world is completely devoid of meaning and value. It is most commonly associated with Camus' philosophy, especially in The Myth of Sisyphus.

What immediately follows from this liberating embrace of ambiguity is the need to adopt what Nietzsche calls "noble morality" as distinct from "slave morality". Very simplistically, slave morality is about adherence to an external normative code, such as that laid out in the Bible. It is called "slave" morality because it involves obedience to someone else's values. Noble morality is the opposite: it involves embracing your nature as a value creating entity and acting with fidelity to your own good and evil: "The most basic laws of preservation and growth require the opposite: that everyone should invent his own virtues, his own categorical imperatives" (Nietzsche 1885/1990, p. 134). A noble's ethical sense is attuned primarily to whether they are transgressing their own values rather than whether they are transgressing other people's values (1888/2000, p. 495):

The proud awareness of the extraordinary privilege of responsibility, the consciousness of this rare freedom, this power over oneself and over fate, has in his case penetrated to the profoundest depths and become instinct, the dominating instinct. What will he call this dominating instinct, supposing he feels the need to give it a name? The answer is beyond doubt: this sovereign man calls it his conscience.

Nietzsche talks often of "profound selfishness". What he means is that you owe allegiance to your own values first and foremost. It does not mean that you don't care about society's values or those of your particular subculture. You can certainly adopt these as your own and enforce them upon yourself (most people do at least to some extent), but you are the ultimate authority over what is right and good and nothing can absolve you or rob you of this responsibility. The French existentialists would later summarise this in one of their maxims: "man is condemned to be free". In the writings of Nietzsche and the French existentialists this freedom is both the source of despair and the font of tremendous happiness and wellbeing provided the individual can overcome that despair. 
It is also worth noting that Nietzsche's selfishness is not about repudiating altruism and otherregarding conduct. It is simply about locating the reason for altruism within the individual's own ethical rubric because it thereby becomes a source of individuation (Nietzsche 1881/1996, s. 103):

It goes without saying that I do not deny_ - unless I am a fool — that many actions called immoral ought to be avoided and resisted, or that many called moral ought to be done and encouraged - but I think the one should be encouraged and the other avoided for other reasons than hitherto.

An absolutely critical aspect of noble morality and the coalescence of being more generally is integrity. Integrity is "the right to make promises". Nietzsche (1888/2000, book 2, s. 1). Nietzsche is here alluding to the problem, first identified by Kierkegaard, that if the individual is the source of values then these values are open to capriciousness. What is to stop the individual changing their mind at the moment of decision? Our past self does not exert any binding power over our present self because consciousness always allows us to stand apart from it. Integrity as a virtue is a way out of this problem because it discourages the individual from contravening their avowed values. The strength of integrity lies in the fact that it is necessary if individuals want to achieve wellbeing. Integrity is required for seriousness and seriousness is required for wellbeing, so individuals have a self-interest, a privately beneficial reason, to uphold their values. ${ }^{22}$ Nietzsche makes this point more explicitly when he says that conscience is "To possess the right to stand security for oneself and to do so with pride, thus to possess the right to affirm oneself..." (1888/2000, p. 496). Affirmation is critical to wellbeing, and without integrity one cannot affirm oneself because one's commitments are loose. De Beauvoir (1947/2002, p. 27) makes a similar point when she says that "to will is to engage myself to persevere in my will".

\footnotetext{
22 This idea has profound implications for meta-ethics that have not been fully appreciated by philosophers to date, but the present work is not the place to elaborate. However, it is worth noting the following passage from Sartre (1943/2005, p. 646), which more fully explains how and why existentialist ethics moves away from the traditional notion of ethical behaviour as "disinterested conduct". In this philosophy, individuals are the source of (other-regarding) values, and they affirm and live by these values in order to achieve wellbeing. Ethics is then best understood through the prism of self-interest and psychic payoffs to being a good person.

Thus existential psychoanalysis is moral description, for it releases to us the ethical meaning of various human projects. It indicates to us the necessity of abandoning the psychology of interest along with any utilitarian interpretation of human conduct - by revealing to us the ideal meaning of all human attitudes. These meanings are beyond egoism and altruism, beyond also any behaviour which is called disinterested. Man makes himself man in order to be God.
} 
Nietzsche makes one final comment about integrity that is worth elaborating on. He says (Nietzsche 1888/2000, p. 494) that:

Man himself must first of all have become calculable, regular, necessary, even in his own image of himself, if he is to be able to stand security for his own future, which is what one who promises does!

The reference to "his own future" speaks to the relationship between integrity and wellbeing. Perhaps more important though is the notion of becoming "calculable, regular, necessary, even in his own image of himself". This is reminiscent of the Hellenic maxims: "know thyself" and "become who you are". If you know yourself then you are calculable — rationally accessible - in your understanding of yourself. If you are proceeding to be the person who you claim to be and want to be by affirming your values through integrity then your identity is a necessity - you cannot deviate from it because then you would lose your integrity and, in so doing, lose your "self" and your wellbeing, which will dissolve in despair. There is an element here even of the Aristotelian notion of living "in accordance with reason". You have reasons for why you value the things that you do and you live in accordance with those reasons through integrity. If the reasons upon which your values are based are confronted by other reasons that some more salient part of your identity finds convincing then you will have to adjust your identity. But until such times you will persevere in your will for the sake of goodness and your own happiness.

Integrity is the bedrock of seriousness in existentialism and an important element in the process by which wellbeing is achieved. The other crucial element is what the French existentialists called "the disclosure of being". This is where one's identity, one's "being", is revealed (disclosed) in the world by our actions and in the impressions and assessments of others. By this revelation, one's being “exists" for a moment (De Beauvoir 1947/2000, p. 30):

My freedom must not seek to trap being but to disclose it. The disclosure is the transition from being to existence. The goal which my freedom aims at is conquering existence across the always inadequate density of being.

Disclosure is important because in it we are revealed as who we are in actuality. If what is disclosed aligns with who we want to be then we experience a rush of self-actualisation. If what is disclosed diverges from our ideal self then we will experience depression. Repeated disclosures of who we want to be gives us the sense that we are moving towards - coalescing 
towards - our ideal self. This alleviates anguish for two reasons. First, we get the sense that our commitments (to ourselves especially) are trustworthy - we have the right to make promises. This means that we are not entirely cut off in the moment of decision from our prior commitments. Second, as coalescence progresses we disclose an increasingly broad, refined, deep and, critically, consistent identity across a range of environments and circumstances. By this we come to have a clearer sense of who we are, thereby mitigating the anguish that comes with not knowing our identity.

What remains to be explained is how Nietzsche and the French existentialists thought we could overcome nausea. The first step is to accept that transcendental meaning is not possible. Things are only meaningful because we care about them (De Beauvoir 1947/2002, p. 15):

Man exists. For him it is not a question of wondering whether his presence in the world is useful, whether life is worth the trouble of being lived. These questions make no sense. It is a matter of knowing whether he wants to live and under what conditions.

Though they never say it explicitly, the source of meaning in existentialist thought is close or at least related to intrinsic motivation. There are things that we care about because we, in a sense, can't help caring about them. Arguments about the inherent purposelessness of the universe bounce off intrinsic motivation. We approach some values, activities and goals through a kind of primal movement of the spirit. The meaningfulness of these things to us requires no explanation (De Beauvoir 1947/2002, p. 158):

Let men attach value to words, forms, colors, mathematical theorems, physical laws, and athletic prowess; let them accord value to one another in love and friendship, and the objects, the events, and the men immediately have this value; they have it absolutely. It is possible that a man may refuse to love anything on earth; he will prove this refusal and he will carry it out by suicide. If he lives, the reason is that, whatever he may say, there still remains in him some attachment to existence; his life will be commensurate with this attachment; it will justify itself to the extent that it genuinely justifies the world.

As with seriousness, the capacity of these values to ward off nausea is grounded in their ability to bring us happiness. When we pursue our intrinsically motivated goals we 
experience the feeling of purpose. When we successfully pursue these goals we experience "happiness" (De Beauvoir 1947/2002, p. 136):

However, it must not be forgotten that there is a concrete bond between freedom and existence; to will man free is to will there to be being, it is to will the disclosure of being in the joy of existence; in order for the idea of liberation to have a concrete meaning, the joy of existence must be asserted in each one, at every instant; the movement toward freedom assumes its real, flesh and blood figure in the world by thickening into pleasure, into happiness. If the satisfaction of an old man drinking a glass of wine counts for nothing, then production and wealth are only hollow myths; they have meaning only if they are capable of being retrieved in individual and living joy. The saving of time and the conquest of leisure have no meaning if we are not moved by the laugh of a child at play. If we do not love life on our own account and through others, it is futile to seek to justify it in any way.

The essence of the existentialist approach to nausea is simply to observe that caring about something has the power to make that thing meaningful. Humans are wired to care about things, to imbue them with symbolic significance, to see them as meaningful and their promotion and purposeful. This is the neural architecture that allowed us to craft the great religions of the world. Meaning is contrived, but admitting that doesn't rob it of its power. Things matter because we care about them. That is enough (De Beauvoir 1947/2002, p. 159):

The fact remains that we are absolutely free today if we choose to will our existence in its finiteness, a finiteness which is open to the infinite. And in fact, any man who has known real loves, real revolts, real desires, and real will knows quite well that he has no need of any outside guarantee to be sure of his goals; their certitude comes from his own drive.

\section{Conclusion}

Existentialism emerged in response to the profound challenge of ambiguity that confronted European society in the wake of the enlightenment. It analysed three problems: Nausea, seriousness and anguish. Nausea is today studied in the wellbeing literature under the rubric of meaning and purpose. Seriousness is being studied by psychologists working on moral 
cognition and by a range of social scientists studying the role of culture in perpetuating and enforcing norms, but it has not made its way into the wellbeing discussion. The notion of identity and the anguish that makes it hard to achieve are almost completely absent from wellbeing discussions outside of adolescent psychology, though the notion of "finding yourself" remains a favourite of pop-psychology and self-help books. Existential philosophy can today add texture to the scientific study of these phenomena. More importantly, it can highlight that success in these normative dimensions of wellbeing will require, for many people at least, negotiating the very tricky issues of nihilism and subjective values. Meaning, seriousness and identity are not things that we can just choose to have one day. They require patient, thoughtful exertion over a sustained period of time. This exertion is very difficult if one doesn't have all the necessary philosophical tools. Existentialism provides many of these tools. On some subjects, especially the issue of relativism, existentialism was an incomplete philosophy, but this is not the place to dwell on these matters. Suffice that I have hopefully demonstrated that the existential problems identified at the start of this chapter are an important part of wellbeing that should not be overlooked, and that existential philosophy provides at least the beginnings of a powerful theory of how self-actualization can overcome these problem. In the next chapter I complete this theory of self-actualisation - the coalescence of being - drawing on a wide-range of literatures in psychology. 


\section{Chapter 9}

\section{The Coalescence of Being}

They say that to know oneself is to know all there is that is human. But of course no one can ever know himself. Nothing human is calculable; even to ourselves we are strange.

- Gore Vidal, Julian

\section{Introduction}

This chapter outline the coalescence of being in full. The coalescence of being is a model of the process by which the outcome of SWB as modelled in the production function is achieved. Recall from chapter 3 that understanding this praxis of SWB is critical for understanding SWB holistically. There are two reasons for this. First, if you pursue SWB the wrong way you will never attain it. By extension, knowing about the outcome or state of SWB is insufficient for advising people, in a clinical or policy context, on how to be well. The coalescence of being describes the right way of pursuing SWB. For the philosophers: the prudential good describes not just an outcome but also a process. Second, training an eye on praxis illuminates the extent to which the different dimensions of SWB are interlinked and hence why they need to be studied with at least an awareness of how they interact.

I outline the coalescence of being in the following stages. I begin with the core of the process, which is the harmonisation of the actual, ideal and ought selves. This involves both introspection and engagement with the external world and self-discovery and self-creation. It is guided by affective signals. I draw on self-determination theory to flesh out what each of these selves represents and to explain the motivational underpinnings of coalescence. I show how coalescence brings about the conditions for zest and flow. Having sketched the basic principles of coalescence, I move to flesh out the details. The first question to answer here is where intrinsic motivations come from? I find answers in the developmental psychology 
literature. I then go into depth on the nature of goal pursuit. I discuss why self-concordant goals make a greater contribution to SWB than just any old goal, and also how we can know that we have achieved a goal. The discussion in this section extends the existentialist's notion of the disclosure of being, linking it with more recent empirical work in social selfverification. Next I turn to the automatisation of behaviour, which is one of the principle means by which the coalescence of being dampens anguish. This discussion draws heavily on the literature in personality systems integration theory. In the final section I return to the wellbeing production function, pointing out and explaining how the coalescence of being improves each aspect of SWB represented in the function. This section begins with a lengthy discussion of relatedness. Here I discuss the role of collectives in the coalescence of being and respond to concerns about whether coalescence is a narrowly individualistic doctrine. It is not.

\section{The Core of Coalescence - Integration and Motivation}

The innermost core of the coalescence of being comes from self-discrepancy theory (Higgins 1987). The basic idea of self-discrepancy theory is that people will be distressed by divergences between their actual self (the person they are), their ideal self (the person they would like be) and their ought self (the person they have a responsibility to be). This distress will be communicated to them by negative affect, which can reach depression levels if discrepancies are large, acute or unaddressed. Higgins theorised that discrepancies between actual and ought selves would lead to agitation while discrepancies between actual and ideal selves would give rise to feelings of dejection. Resolving discrepancies would reduce these negative feelings and be accompanied by positive affect. Self-discrepancy theory is a more sophisticated expression of the core process of the coalescence of being. Instead of harmonising who you are and who you want to be, you harmonise your actual, ideal and ought selves. Failure to do this will result in the preponderance of negative affect, while success will result in a preponderance of positive affect.

These basic ideas are supported by empirical evidence, though the association of agitation with ought-self discrepancy and dejection with ideal-self discrepancy has been hard to measure cleanly (Silvia and Eddington 2012). This is in large part because depression and anxiety are often correlated, so people are both dejected and agitated, and discrepancies with ideal and ought selves are similarly highly correlated, often larger than 0.7 (Gonnerman et al 
2000; Phillips and Silvia 2010; Tangney et al 1998). This means that people who don't converge with their ideal self usually also don't converge with their ought self. The constructs have been demonstrated to be meaningfully different, but it seems that attaining convergence with either of them requires the same skill set across both constructs.

Another important finding from recent empirical work is the discovery of a fourth construct: the undesired self. This is 'a representation of the self at its worst, sometimes called the feared self', which is 'a fundamental avoidance goal, a self that people strive to prevent' (Silvia and Eddington 2012). On average, discrepancies from undesired selves have much stronger relationships with emotion than ideal or ought self discrepancies. However, ideal and ought self discrepancies exert a more powerful emotional effect the further an individual is from their undesired self (Carver et al 1999; Heppen and Ogilvie 2003; Woodman and Hemmings 2008). In practical terms, this means that escaping anxiety and depression is often a function of transcending your feared self, whereas the movement towards positive emotion requires moving towards ideal and ought selves. More colloquially, the first step on the road to happiness is to ensure you do not become what you hate. The majority of the path is then made up of moving towards what you love. As Adam Smith said, 'man naturally desires, not only to be loved, but to be lovely; or to be that thing which is the natural and proper object of love' (Smith 1759).

An important element of self-discrepancy theory is the idea that the self is a dynamic, integrative system that develops in interaction with its environment. This development is punctuated by affective signals (Showers and Zeigler-Hill 2012, p. 115). There is an executive function that is capable of reflexive thinking, often provoked by emotional messages from the broader mental system, but the self is fundamentally composed of several parts that don't necessarily enter consciousness or act in harmony (Damasio 2010). This is inline with contemporary psychological research into the nature of self. A definition of the self that emerges from this research is the following (Morf and Mischel 2012, p. 22):

The self and its directly relevant processes (e.g. self-evaluation, self-regulation and self-construction) may be conceptualised fruitfully as a coherent organisation of mental-emotional representations, interacting within a system of constraints that characterise a person (or a type) distinctively...but it is also a motivated, proactive knowing, thinking, feeling action system that is constructed, enacted, enhanced and maintained primarily in interpersonal contexts within which it 
develops. Through this organised system the person experiences the social, interpersonal world and interacts with it in characteristic self-guided ways, in a process of continuous self-construction and adaptation.

It seems Kierkegaard was vaguely correct in his description of the self as a relation that relates to itself, but neither Kierkegaard nor Norton was correct in orienting his philosophy completely towards the internal or external. Modern psychological science stresses that the self is determined both volitionally and by its environmental context. This take on self also suggests that existentialism's general neglect of positive emotion was unwise, because this leaves out the role of positive affective signals in motivating the individual and confirming that their current behaviours and beliefs are self-concordant.

Self-determination theory (SDT) provides a great deal of clarity regarding what the actual, ideal and ought selves specifically are that is grounded in a theory of motivation, which in turn leads nicely into the literature on goal strivings. In SDT, the actual self is characterised by intrinsic motivation. Intrinsically motivated activities are (Deci and Ryan 2000, p. 223):

those that are freely engaged in out of interest without the necessity of separable consequences, and, to be maintained, they require satisfaction of the needs for autonomy and competence...they are activities that people do naturally and spontaneously when they feel free to follow their inner interests.

Intrinsically motivated activities are thus those that are freely and autonomously engaged in for their own sake. They aren't necessarily productive, beneficial or good. One's actual self might be quite inclined to be a couch potato, for example.

The ideal self in SDT is composed of values, goals and behaviours that the individual identifies with or is in the process of integrating into their actual self, while the ought self is composed of introjected values, goals and behaviours. Identified, integrated and introjected here referred to different points along the continuum of motivation proposed by SDT, which runs from intrinsic at one end to extrinsic at the other. An entirely intrinsically motivated activity is part of the actual self (though it may not originally have been innate). At the other end of the spectrum are entirely extrinsically motivated acts done under duress.

Recall that the process by which behaviours move from external regulation through introjection and identification to integration is internalisation. This concept contains both social and egoistic elements. Individuals exist in a social world saturated with values. The 
desire for relatedness encourages individuals to comport themselves towards those groups with which they feel the greatest kinship. The greater the diversity of groups to which an individual can comport, the more individualistic this choice can be. However, it will also be more difficult, because in the early stages of developing a social identity the individual does not know themselves very well, and may well approach groups that are wrong for them. This is common in adolescence, wherein teenagers crave social acceptance but often bounce around different cliques looking for the one that fits them best. When the individual and group are a bad fit, the individual can only ever be extrinsically motivated towards the mores of the group. However, when the group is a good fit, this does not mean that the individual will immediately be intrinsically motivated to accept all of the group's mores. Instead, the individual will begin by identifying with these mores and gradually internalise them until they are intrinsically motivated. Deci and Ryan (2000, p. 239) summarise this as follows:

...research on internalisation of extrinsic motivations highlights the human readiness to internalise ambient values and regulations. Yet to fully integrate such values and regulations, and thus to become self-determined with respect to them, people must grasp their importance and synthesise their meaning with respect to other values and motivations...the holistic processing and self-compatibility checking that is necessary to act with self-concordance requires the experience of freedom from rejection by others, from indicators of incompetence, and from excessive pressures. In this sense, supports for relatedness, competence, and autonomy allow individuals to actively transform values and regulations into their own, and thus to be more self-determined.

What this means for the practice of the coalescence of being is that the actual self parameterises the individual's choice of group through the competence and autonomy dimensions. If you are dumb, you can't join the mathletes no matter how much this is your "ideal" group. Similarly, if you dislike exercise, the football team will have a hard time convincing your 6'6" frame to play linebacker no matter how much they want you in their group, because you will only ever be extrinsically motivated to play football.

An integration of SDT and terror management theory (TMT) by Pyszczysnki et al (2012) links the internalisation process to zest and flow. Zest is a notion developed by philosopher Bertrand Russell (1961/2006, p. 110) in his The Conquest of Happiness. He does not provide a succinct summary of it, but the following will do: 
What hunger is in relation to food, zest is in relation to life. The man who is bored with his meals corresponds to the victim of Byronic unhappiness. The invalid who eats from a sense of duty corresponds to the ascetic, the gormandiser to the voluptuary. The epicure corresponds to the fastidious person who condemns half the pleasures of life as unaesthetic. Oddly enough, all these types, with the possible exception of the gormandiser, feel contempt for the man of healthy appetite and consider themselves his superior. It seems to them vulgar to enjoy food because you are hungry or to enjoy life because it offers a variety of interesting spectacles and surprising experiences. (Emphasis added).

Zest corresponds to the spontaneous enjoyment of life for its own sake. It is intimately connected with intrinsic motivation. It has parallels with flow, but does not necessarily involve high challenge and high skill. The internalisation process transforms identified activities and values into intrinsic ones over time. Therefore, as the individual steadily orients themselves away from extrinsic activities and values and towards identified ones, intrinsic motivations and values come to preponderate in the individual's psyche and among their daily activities. This brings about zest — the spontaneous enjoyment of life as an intrinsically rewarding experience.

Because identified behaviours and values still contain an element of extrinsic regulation and are thus cognitively taxing to engage in, it is effortful to transition them to the point where they are intrinsic. If one has the requisite skill to do so, one satisfies all the condition for flow as a feeling that permeates one's life: high challenge, high skill, intrinsically motivated activities across a range of different life domains. The comportment towards the ideal self over time is an iterative process that involves reassessing the ideal self and actual self concepts following successes and failures. This is effectively a calibration of skill (actual self) to challenge (ideal self) over time as we abandon excessively challenging goals and replace them with ones more suited to us.

As such, the coalescence of being brings about flow and zest by way of internalisation. As Pyszczynski et al. (2012, p. 385) explain:

In this context, integrative processing entails changing one's existing psychological structures to accommodate the new information or experience, and is the mechanism through which growth, learning and change within the individual occurs. The heightened positive affect or exhilaration resulting from 
integrative processing acts as an incentive for one to approach challenging tasks in the future and as a reinforcer for such engagement once it has occurred. Thus, it is through the process of integrative activity that occurs in challenging situations rather than the products or outcomes of such activity that intrinsic motivation is generated.

This synthesis of self-determination theory, self-discrepancy theory, terror-management theory, flow, zest and the existentialist's notion of disclosure is the core of the coalescence of being. The following sections of this chapter build on this foundation. Up next is an analysis of how the coalescence of being integrates the innate self at the heart of Norton's philosophy. The text then moves on to a more detailed analysis of how achieving the ideal self links into the literature on goal strivings.

\section{The internal and the external}

One question that emerges out of the preceding analysis is where initial intrinsic motivations come from. Are they innate? SDT provides some guidance on this in their definition of self (Deci and Ryan 2000, p. 248):

Our concept of self, because of its organismic basis, begins with intrinsic activity and the organismic integration process - that is, with the innate tendencies of human beings to engage in interesting activities and to elaborate and refine their inner representation of themselves and their world. The activity and integrative tendency move the organism toward a more unified set of cognitive, affective, and behavioural processes and structures...the inherent tendency for activity, the integrative process, and the fundamental needs are all aspects of one's nascent self, and gradually the self is elaborated and refined through the integrative process. As such, behaviours that are motivated by regulations that have not been fully integrated into the self are not considered self-determined.

So the tendency to engage in interesting activities is innate, as is, presumably, what each individual finds "interesting", at least initially, but how the individual develops over time depends on environmental interactions. This jibes with the analysis in the previous chapter on eudemonism. It is reasonable to conjecture that we are born with certain innate parameters and dispositions that incline us towards particular values, behaviours and activities, but that 
the exact expression that these take over the course of our lives will be determined in large by how we respond to environmental constraints and prompts.

Empirical research on the emergence of self in adolescence provides a more rigorous understanding of how the nascent self emerges and becomes accessible to consciousness. This research makes use of some early ideas in psychology from James (1980) and Piaget (1960). James' distinction between the I-self as the actor, knower or recogniser and the meself as the object of one's knowledge remains fundamental in the analysis of emergent identity, as does Piaget's concept of egocentrism, which refers to a singular focus on the self (Harter 2012). The antithesis of egocentrism is perspective taking, which is the ability to take the viewpoint of others using mental images generated in the mind.

Toddlers have self-recognition (Rochat 2003). For example, when placed in front of a mirror with a spot of rouge on their nose, they rub the rouge, indicating that the colouring violates their understanding of what they look like. Very young children are also capable of describing themselves, their feelings and their ownership of objects (Kring 2008; Thompson 2006). Toddlers also demonstrate agency and a rudimentary I-self in the sense that they recognise themselves as the same entity across time (Lewis 2008; Nelson 2003). These imply a degree of autobiographical memory (Fivush and Haden 2003). Young children also understand that they are a person recognised by others. This is the basis for the future development of perspective taking, a capacity toddlers lack (Rochat 2003; Thompson 2006).

Children develop metacognitive awareness around 5 or 7 (Rochat 2003). This is the ability to think reflexively about one's own thoughts. Perspective taking improves rapidly between the ages of 8 and 10, improving self-awareness and preparing the child for the intense socialisation of adolescence (Selman 2003). Social awareness develops next, and the early teenage years are unsurprisingly marked by heightened self-consciousness and concern with the appraisals of others. Multiple self concepts are also the norm in adolescence, and thinking tends to be extremely categorical and compartmentalised (Harter 1999). Individuals then try to harmonise these cleavages over time (Higgins 1991). This harmonisation begins in earnest in mid-adolescence, around 13-15 years old, in which there is 'a dramatic rise in the detection of contradictory self-attributes that lead to conflict and confusion' (Harter et al 1997)..$^{23}$

\footnotetext{
${ }^{23}$ Note how distinct this is from Norton's theory. He posited that adolescents find themselves misunderstood by others and subsequently embark on a quest of self-discovery. Contemporary psychological research paints a
} 
A critical skill for the harmonisation of self-concepts emerges in late adolescence, namely the ability to "construct higher-order abstractions that provide a meaningful integration of single abstractions that previously represented contradictions" (Harter 2012, p. 706). An example is resolving an apparent contradiction between a studious self-image and lackadaisical behaviour in practice by asserting that you only study what you care about. Late adolescents are context sensitive when it comes to which aspects of themselves they present to the social realm. The ability to construct higher order abstractions allows many beliefs, values and standards to be clearly articulated and subsequently internalised, leading to more selfcoherence and a greater sense of agency (Harter, ibid.).

From the analysis above it should be clear that we arrive at full consciousness with some identity already. The extent to which this is innate or genetic versus environmentally determined is somewhat beside the point. What matters is that we always have an "actual" self, and that in our earliest encounters with it we apprehend it as discordant, confused and vague. Using our emerging sense of agency, we explore different facets of this actual self in the social world, gradually developing conceptions of our ideal and ought selves and comporting ourselves towards them. A greater degree of self-coherence comes about over time as a result. This brings us back to the basic point about self, which is that it is a reflexive relation that is constantly under construction, largely through interaction with the social world: "the self is an interpersonal self-construction system" (Morf and Mishel 2012, p. 27).

\section{The vehicle of coalescence: goal setting, achievement and recalibration}

The coalescence of being is closely related to the psychology of goal setting. Who you want to be is a goal, and attaining it involves goal setting and approach. There is a fairly large literature empirically demonstrating that people with clear goals on average have higher wellbeing than people who are, in a sense, aimless (Deci and Ryan 1985; Oyserman and Markus 1990; Locke and Latham 1990). However, this average effect obscures a lot of very interesting detail pertaining to authenticity. For example, Emmons $(1986,1999)$ observed that it is important not just to have goals but to attach some personal importance to them. This implies goals must be autonomously chosen and meaningful rather than simply interesting. In line with this, Sheldon has consistently replicated results showing that goals have stronger

different picture: adolescents cannot understand themselves and subsequently set out on a quest that is part selfdiscovery, part self-creation and part self-synthesis. 
effects on wellbeing when they are self-concordant (Sheldon and Kasser 1995; Sheldon and Elliot 1998, 1999; Sheldon and Houser-Marko 2001; Sheldon 2002).

There are two requisites for a goal to be self-concordant. The first is that it be pursued autonomously in the sense that the individual is intrinsically motivated. This pursuit might begin through introjection, but the wellbeing payoffs are strongest in the latter stages of internalisation. The second is that goals are aimed at intrinsic pursuits of personal growth, affiliation and community rather than extrinsic pursuits contingent to activities themselves, like financial success, image and popularity (Sheldon et al 2004; Kasser 2003). This is in-line with the theoretical postulates of the coalescence of being and SDT, which both stress that goals must be authentic in the sense that the individual moves towards them volitionally and out of an interest in the associated activities. It is also in line with the interpersonal dimension of the coalescence of being, which emphasises the need to find camaraderie (affiliation) and harmonise the self-as-individual with the self-as-member of various collectives.

A critical link between the goal setting literature, the coalescence of being and positive psychology is the finding that people only have strong affective responses to goals that relate to ego and identity. Such goals are sometimes referred to as contingencies of self-worth (Crocker and Park 2012). Successes and failures in goals that are not ego-involved do not provoke affective responses, but people have few such goals (ibid. 2012, p. 322). The differences in affective response to success and failure in contingent and non-contingent domains can be stark. For example, figures 9.1 and 9.2 below (reproduced from Crocker and Park 2012, p. 312-313) show self-esteem fluctuations for a student with little egoinvolvement in academic goals and a student with a large amount of ego-involvement in academic goals. The lines track their self-esteem across a time span in which they receive acceptance and rejection letters from graduate programs.

The self-esteem of the student oriented towards academic goals is far more volatile in response to self-relevant information in the form of acceptance and rejection letters. Meanwhile, the self-esteem of the student with little ego-involvement in academic goals does not seem to fluctuate at all in response to acceptance and rejection letters. 
Figure 9.1-Student with little ego involvement in academic goals

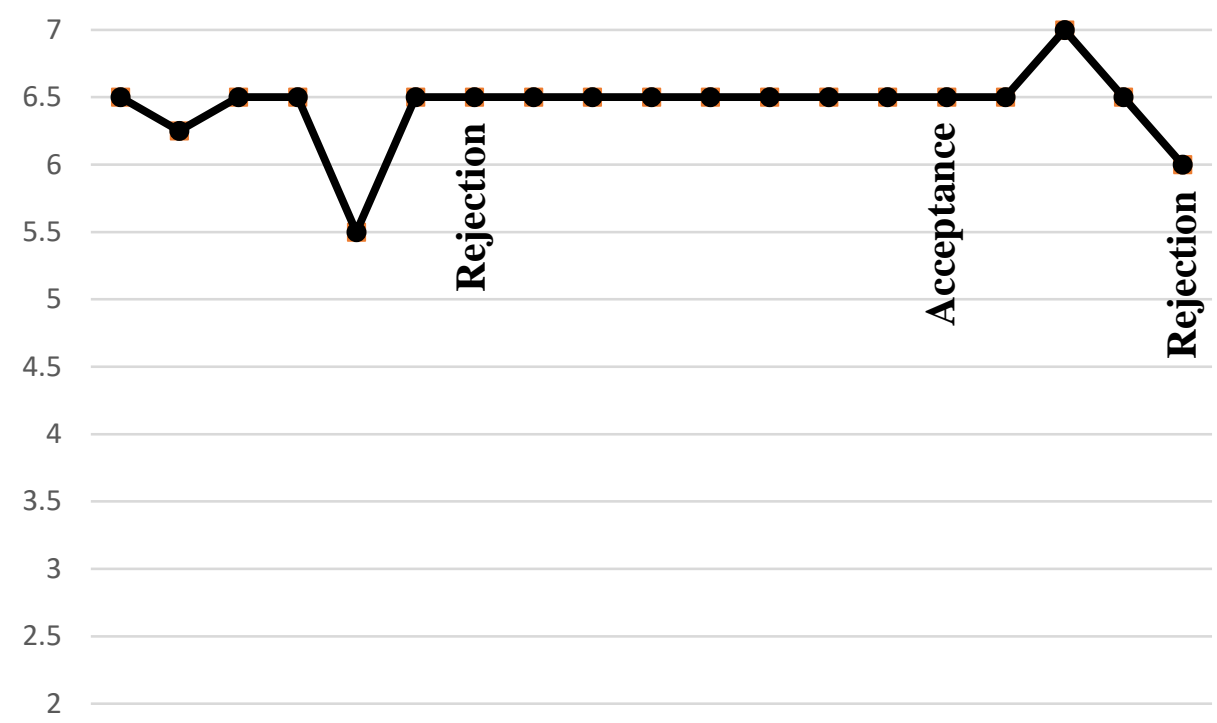

Figure 9.2-Student with substantial ego-involvement in academic goals

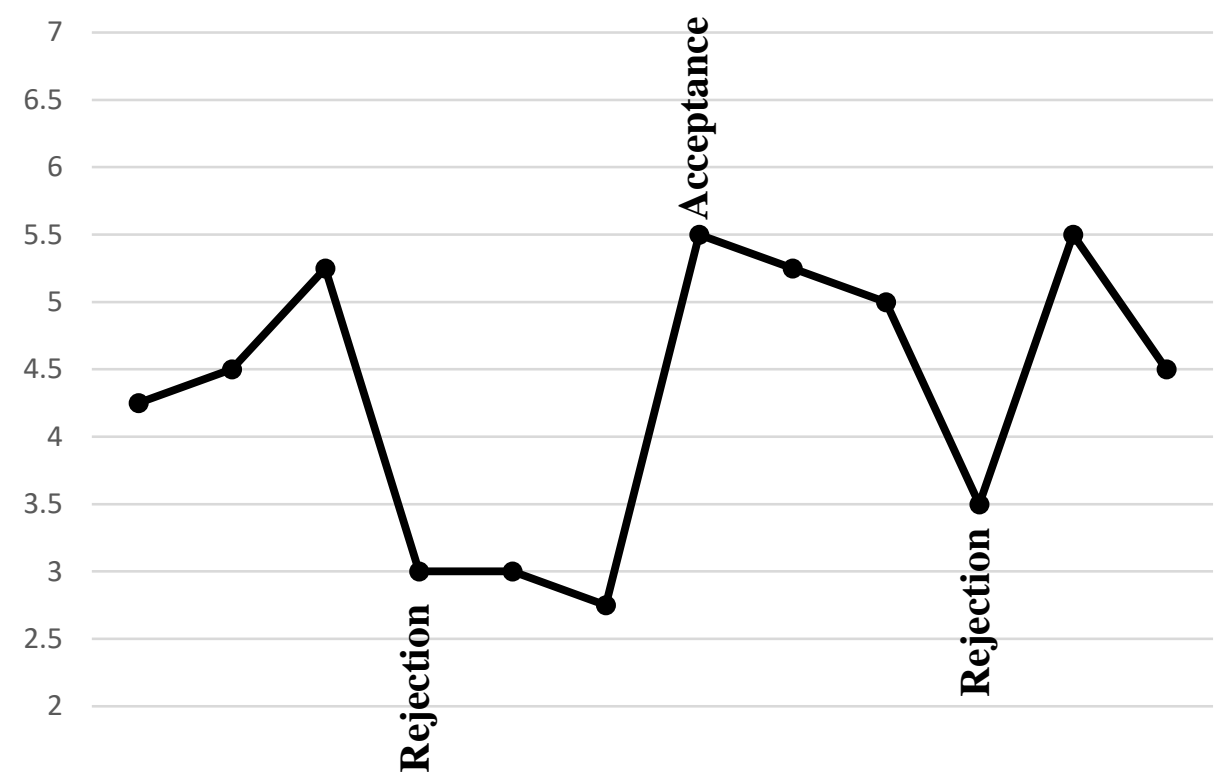

The relationship between contingencies of self-worth is clear in the written responses of participants in the study above. Three students low in academic orientation wrote (reproduced from Crocker and Park 2012, p. 15):

Getting into graduate school is a formality. It is a mere reminder of potential, rather than a reflection of hard work. It signifies a long, arduous road ahead in academia. 
It means that I have been granted an opportunity to gain the knowledge and skills I need to be a competent and successful researcher. I will also be able to experience a different area of the country and make a fresh start somewhere else.

It really would not reflect on me as a person, but it would just be an accomplishment for me to be able to move on to the next step toward a career.

Graduate school obviously has little relationship to these people's sense of who they are, and so they are unperturbed by acceptance and rejection letters. In contrast, consider the following responses from participants with a strong ego-involvement in academic goals:

Getting into grad school (especially a really good one) would show me that I am one of the best students of an even more select group of students.

Getting into graduate school would mean that I am truly a scholar. It would mean I'm intelligent, hard-working and a logical thinker. It would mean I can now be respected for being a good thinker.

It means that my hard work payed [sic] off, and would mean that at least one grad school recognised that I am a brilliant and motivated student. In other words, it would reaffirm what I already know.

In all three cases the language of confirmation is present, as is the language of belonging. Putting these written reports together with the graphs of self-esteem, the link between the coalescence of being and goal achievement emerges. When we are disclosed as our ideal selves in our impact on the world and the eyes of others we coincide with ourselves. When we are disclosed as something other than our ideal selves we experience an existential fail instead and feel worthless (some self-compassion might be helpful here). The evidence above - two graphs and a few excerpts - is anecdotal, but data from the full samples in Park and Crocker's studies follow the themes outlined above. They summarise (2012, p. 315): 
As expected, within-person analysis of acceptances and rejections from graduate programs indicated that affect and self-esteem rose and fell together...furthermore, the more participants staked their self-worth on academic competence, the stronger their affective reactions to acceptances and rejections.

An important point to underline here is the link between contingencies of self-worth, selfesteem and affect. Failure in non-contingent domains has limited effects on affect and selfesteem, and success in non-contingent domains provokes mild positive affect but no appreciable change in self-esteem. By contrast, success and failure in contingent domains provokes large changes in self-esteem and highly correlated commensurate changes in affect (Crocker and Park 2004). The implication is that moments of coalescence lead to large changes in emotion, while goal setting and achievement that is not self-congruent has little effect on emotion. Put another way: affective signals guide the process of self-actualisation. King and Hicks (2012, p. 137) make this point eloquently:

These results suggest that to some extent, more global-level analyses gloss over the dynamic between positive affect and sources of meaning on a more micro level. It appears that positive affect may, at any given moment, play a role in enhancing the experience of meaning provided by more conceptually relevant sources of meaning, lending hedonic reinforcement to these eudaimonic endeavours. Thus positive affect may be the affective reward that accompanies other relatively costly meaningful behaviours (e.g. flow, altruism). Indeed, positive affect may play a role in developing, supporting and maintaining the relations between the experience of meaning in life and these central sources of meaning.

It is by introspecting on affective signals that we are able to interpret what values and behaviours are self-concordant and why. It is thus critical to study hedonia, eudaimonia and despair together if we are to understand SWB in a holistic way, because these dimensions of SWB are interlinked and interdependent.

Another important finding from research into contingencies of self-worth is that few people have no contingencies of self-worth. For example, in a study of college freshman, only 4 per cent of students scored 3 or lower on seven 1-7 scales designed to assess what goal-related factors mattered to people's self-worth, and these 4 per cent may well have had contingencies of self-worth not captured by the survey (Crocker 2002). This suggests that positive affect 
has a very substantial subjective, existential component that will be overlooked if affect is studied using the average effect of emotional interventions across large samples.

While the incidence from the contingencies of self-worth suggests that clear, authentic goals are important to SWB, evidence from other lines of research suggests that it is wise not to be too specific in one's goals. Pyszczynski et al (2012) posit that making one's self esteem contingent on more abstract standards can make them easier to satisfy. They give the example of being a top athlete versus being an Olympic medallist. There is not necessarily a difference in degrees here, as an Olympic medallist is certainty a top athlete. However, someone with the more abstract goals could derive a lot of confirmation and satisfaction from simply qualifying for the Olympics, while the later requires something much more specific that can be interrupted by unrelated issues, like injury. This recommendation of abstraction is supported by Freitas et al (2001), who report six empirical studies showing that people engage in more self-esteem defence when they construe their actions at more concrete as opposed to more abstract levels. Another bit of supportive empirical evidence comes from Vess et al (2011), who found that participants induced to adopt an abstract orientation are less reactive in terms of changes in self-esteem to negative feedback. However, Pyszczynski et al (2012) also acknowledge that abstract contingencies of self-esteem can be harder to satisfy precisely because the requisite conditions are vaguer, which makes self-assessment and the tracking of progress harder. Some balance must be achieved between not setting oneself up to fail while nonetheless delineating clear targets that can provide feedback along the way to one's ideal self.

How do we know we have achieved a goal? What experiences prompt the disclosure of being? A relatively straightforward source of information is objective measures. For example, if one's goal is to be able to run fast, then gradually reducing your personal best time on the $100 \mathrm{~m}$ dash will give the sense that you are getting faster. There are typically a range of objective metrics that can be associated with any desired identity.

Another source of evidence is other people, but the disclosure of being becomes more complicated when you move into the realm of social confirmation. There is no shortage of material advising us not to worry about what other people think. But we do worry of course, and rightly so. There is overwhelming evidence that we are prone to self-enhancement in our opinion of ourselves, so it is important that we give ourselves a reality check occasionally with the assessments of others (Sedikides et al. 2005). Furthermore, if we are trying to 
comport ourselves towards a group then it is obviously imperative that we check in with the group occasionally to make sure we're on the right track. This is particularly the case when what we are trying to excel at is relative, such as being the best chess player, or subjectively assessed, as in art circles. People's opinions obviously can wound us and we should be careful and exercise self-compassion when exposing ourselves to negative feedback. But such suffering is also the affective signal to disconnect from inappropriate goals, and such suffering may simply see us move towards healthier social groups (like art circles with tastes closer to our own) so we should not neurotically avoid it. Social dis/confirmation is an important source of evidence about our being.

The analysis above is indirectly supported by a raft of empirical evidence, notably in social self-analysis, which concerns itself with how people evaluate themselves relative to others (Alicke et al 2012). Social appraisals have been found to exert more influence on selfappraisal when the perceiver is considered by the perceived to be relevant to their selfconcept, an in group member, desirable, valued or otherwise important (Wallace and Tice 2012). Neuroscience studies align with this result. They show that when an appraiser is from a group you care about their appraisals of you will activate the self-assessment part of your brain. This is not the case when they are from a group you don't care about or a random stranger (Devos et al 2012, p. 158).

Self-verification theory (SVT) explains how social assessments interact with the coalescence of being to create a self-reinforcing cycle (Swann 2011). SVT conjectures that self-views guide social interaction and, provided they are stable, make an individual's behaviour more predictable to others. This predictability stabilises the way others respond to the individual, which makes it easier to verify one's self-view through social interaction. Stable self-views thus encourage the emergence of a stable, coherent social environment and vice versa, leading to a virtuous cycle wherein both self-concept and social environment become clearer and better fitted to each other. An important, empirically validated hypothesis that emerges from this theory is that people prefer social appraisals that align with their self-view even when these appraisals are negative (Swann and Buhrmester 2012). People move away from both incorrect and correct but negative appraisals over time towards groups that are both affirming and accurate in their social appraisals. 
This chapter has now covered the basic methods of coalescence and provided some detail regarding its praxis through the goal setting literature. From here, the chapter turns to discuss the outcomes of the coalescence of being.

\section{Automatisation and the dampening of anguish}

A major component of the Coalescence of being is the gradual automatisation of decisions over time in many domains. This overcomes anguish, because you rarely need to engage your conscious processing faculties in order to make a decision. Conscious, higher-order decision making is taxing, and thus most efficaciously engaged only at times of substantial ambiguity or complexity. Such ambiguity and complexity is the hallmark of anguish, which intrudes upon us when we don't know what the right choice is. In trying to make that choice, we inevitably articulate a range of reasons for why we should make one decision or another. As the coalescence of being progresses, the reasons why we make particular decisions in certain circumstances and the consequences of those decisions becomes clearer. We can retrieve these reasons more easily from memory, and with habitual use they move almost to the level of instinct, thereby mitigating anguish. In addition, as higher-order cognitive engagement with a range of decision realms becomes less frequent, it frees up conscious attention to be focused elsewhere. The apparatus of conscious processing is consequently more fully available for use at moments of ambiguity where tough decisions need to be made. Such decisions tend to be meaningfully self-defining. With our rational faculties present, personal growth is more likely to result from these decisions because our autobiographical self is there to take note of what we chose and its ramifications.

Research in psychology provides a great deal of insight into how this process works. The first chunk of research comes from sports psychology (Fader 2016). When teaching athletes technique, good coaches will emphasise the distinction between conscious and unconscious processing, or what is nowadays called system 1 and system 2 thinking (or dual process thinking). System 1 is intuitive and instinctive. System 2 is conscious and deliberative (Kahneman 2011). Most things that we do physically are too complex for system 2 to handle, which is why we find it so hard to teach robots to walk (Simon 2017). Consider how a tennis player's arm seizes up for a few hundredths of a second on contact with the ball in order to stabilise the racquet head. If you tried to do that consciously, your arm would seize up long before and for a long time after the ball made contact with your racquet, reducing your 
racquet head speed and the sensitivity of your reaction to contact, ruining your shot.

However, bad habits that system 1 has learnt cannot be corrected without systematic attention from system 2. As a result, a basic principle of teaching technique in sport is to engage the conscious brain during training but have it focus on only one or two technical changes at a time, like keeping the arm loose through the swing or trying to load the wrist more. After a few thousand focused repetitions, the technical change becomes habit and focus can shift to some other technical fix.

In matches, the conscious brain is almost entirely turned off, and instinctive programming is allowed to take over. System 1 is much more capable of making the thousands of calculations per second required to perform complex operations like returning a $120 \mathrm{~km} / \mathrm{h}$ forehand on the run while changing the direction of the ball and reversing its spin. In tennis, the conscious mind should be doing little beyond watching the ball during matches.

The same processes are at work in the coalescence of being. If there is something about yourself that you want to change you will need to engage system 2, and do it repeatedly. Repetition allows habits to form. But system 2 is a limited resource, so there is a degree of opportunity cost involved in using it to resolve one conundrum and not another. Having system 2 engaged all the time will also permeate your life with anguish, so there is a degree of urgency in this committing of things to instinct, and strong incentives to avoid activities that make uncertainty salient, as discussed earlier in terror management theory.

Instinct has the advantage of being able to take in a wider array of information than conscious processing, which can allow it to make better decisions, especially when under pressure (Kahneman 2011). A fascinating finding in this vein is that people introduced to new games often develop winning strategies via system 1 before they are able to articulate what these strategies actually are (Crowley et al 1997). On the flip side, those who can articulate the strategy into system 2 then find it more cognitively accessible later and find it easier to generalise the strategy to similar games (Crowley and Siegler 1999). This has important parallels with the coalescence of being. Consciously processing the reasoning behind our decisions and values before committing it to instinct allows that reasoning to be reengaged later when necessary, and generalised to other domains. By way of a simple example, if one reasons that one won't eat veal because it is a child, then one can generalise this reasoning to suckling pig when it is offered on the menu.

This analysis is supported by studies in goal attainment. As Bargh (2004, p. 388) notes: 
If in a given situation we tend to choose the same goal, the representation of that goal becomes more and more strongly associated with the mental representation of that situation. Thus, eventually that goal comes to be activated automatically when one enters that situation and then operates to guide one's behaviour towards the goal-without one consciously choosing or intending to pursue that goal at that moment, and even without the person aware of the real reasons for his or her behaviour in that situation.

It is also supported by findings in personality systems integration theory (PSI, not to be confused with psi, which concerns telepathy). PSI distinguishes between two different volitional modes. The first, self-control, is responsible for inhibiting impulsive actions and maintaining focus on goals in active memory. The second mode is self-maintenance, which directs activity towards goals that are "either intrinsically appealing or congruent with a multitude of the person's inner values and autobiographical experiences" (Kuhl and Koole 2004, p. 416). Self-control and self-maintenance are functionally opposite, and similar to reflexive and reflective processing (Oyserman et al 2012). Self-maintenance corresponds to instinct in the above analysis, guiding our behaviour automatically towards the reasons, values and goals that define our being. Self-control is closer to conscious processing, helping us to correct bad habits and bring our actual self closer to our ideal self. Internalisation from self-determination theory and the above analysis of automation then explains how behaviours transition from being self-controlled to self-maintained, or in the language of SDT, automatic and automatised:

We argued for a distinction between automatic and automatized behaviours. Automatic behaviours are those that are pushed by controlled processes and whose occurrence is not consistent with one's choices or reflections and cannot easily be brought into the realm of active choice. Automatised behavours, in contrast, are ones that if reflected on, would fit with one's values or needs and could readily be changed when they no longer fit (Ryan and Deci 2004, p. 468).

The automation of behaviour is one aspect of the broader process of refining the self. This was discussed in the previous chapter in terms of the thinning of lines and the integration of multiple selves. The most common way of integrating multiple selves is to determine which self-concept is most appropriate for what context. For example, consider a "superdad" who is a nurturing father at home but a hard-arsed executive at the office (Showers and Zeigler-Hill 
2012). The superdad will need reasoning to determine whether to engage his nurturing persona or his more cutthroat persona. Over time, these reasons will become automatized and his behaviour will habitually swap between the personas as appropriate without effort.

There are two things potentially at issue here. The first is that two different aspects of personality conflict: nurturing and hard-arsed. The second is that aspects of personality that are positive or negative in one context may be the opposite in a different context, potentially leading to dissonance.

A formal but generalised framework for thinking about these issues is provided by evaluative organisation, which operates along a spectrum between evaluative compartmentalisation and evaluative integration (Showers 1992a, 1992b, 2002). Evaluative compartmentalisation sees positive and negative beliefs about the self separated into distinct constructs, with each one containing primarily positive or negative items. For example, an athlete might have two selfconcepts organised around their time at the track and their interpersonal behaviour. The former contains mostly positive self-concepts like fast, talented, high-achieving and hardworking. The latter contains mostly negative self-concepts like moody, distant, boring and one-dimensional. Evaluative integration produces self-concepts that mix such positive and negative categories together so that the negative (positive) concepts are associated with their realistic correlates. In the case of the athlete for example, part of the reason why they are boring is because they are hard working on the track and don't have much time left for culture and socialisation. They are moody because their emotional state depends substantially on the quality of their most recent training session, but their emotional involvement in training is also what makes them high-achieving.

This is reminiscent of Nietzsche's notion of "giving style to one's character". He talked precisely about concerted effort to remove things you don't like about yourself (comportment to the ideal self and away from the feared self), but also the transformation of unavoidable negative qualities into charming quirks. To wit (Nizetzsche 1887/1974, p. 232):

One thing is needed - to "give style" to one's character - a great and rare art! It is practiced by those who survey all the strengths and weaknesses of their nature and then fit them into an artistic plan until every one of them appears as art and reason and even weaknesses delight the eye. Here a large mass of second nature has been added; there a piece of original nature has been removed - both times through long practice and daily work at it. Here the ugly that could not be 
removed is concealed; there it has been reinterpreted and made sublime...for one thing is needful: that a human being should attain satisfaction with himself, whether it be by means of this or that poetry or art; only then is a human being at all tolerable to behold.

The similarities with automatization are also uncanny. "Second nature" is added by way of internalisation and first nature is removed by self-control ("long practice and daily work at it"). Integration is evident in the comment: "here the ugly that could not be removed is concealed; there it has been reinterpreted and made sublime".

\section{How coalescence leads to subjective wellbeing}

The process of the coalescence is now quite well outlined. It is time to bring it back to the components of SWB. I will begin with relatedness.

Healthy, satisfying relationships with others is critical to the wellbeing of the vast majority of people. In SDT, relatedness is one of the three basic psychological needs. There is evidence that the feeling of experiencing the same thing as someone else-I-sharing - is exhilarating and a feeling people seek out (Pinel et al 2004). Collective action and group goal setting and achievement provoke similar feelings. Strong relationships, especially intimate ones, support self-esteem and aid terror-management. Indeed, there is evidence to suggest that self-esteem evolved as an instrument for monitoring one's "relational value to others" (Leary and Baumeister 2000). People seek out group affiliations that are affirming of their self-worth.

At first blush, it might be difficult to see how a paradigm as individualistic as the coalescence of being could nurture feelings of relatedness. But this is to misunderstand the paradigm. The coalescence of being does not obviate against a collective self or against collectivist values. It is not even individualistic. It merely emphasises the following. First, that individual autonomy is a basic psychological need. Any collectivist system that enforces behaviour that it is not autonomous and intrinsically motivated will generate ill-being. ${ }^{24}$ Such behaviour

\footnotetext{
${ }^{24}$ A corollary point is that individual humans feel while a society or culture does not. When a society suffers, the pain is registered by the individuals in that society rather than by the society itself. This must be kept in mind whenever it is claimed that an individual must serve the group. Many people can derive a great deal of utility from the existence of a healthy normative order that they can integrate into. The health of this normative order is thus integral to their wellbeing, and its breakdown will lead to real human misery. But if that normative order is sustained by the suffering of some other people - those who are required to behave in an extrinsically motivated way for the good of the group - then there is a misery trade-off. Liberalism allows for as many people as possible to find their own little subculture, which would appear to maximise utility across society,
} 
results in compliance and protectiveness rather than confidence and pro-social ingratiation (MacDonald and Leary 2012, p. 363). Second, that individuals are the source of values. Societies perpetuate values and enculturate people into normative systems, but it is ultimately individuals affirming themselves that brings value into the world. Values at the social level can make this a very arduous and expensive process for an individual, but it is individuals who make choices between options and thereby demonstrate value. Third, that consciousness allows the individual to stand psychically apart from their peers and make decisions. Indeed, they cannot lose themselves in a collective - this would be bad faith. Individuals are ontologically free.

Social interaction and dynamic engagement with social values is fundamental to the coalescence of being, as outlined in the discussion of internalisation above in the section on self-determination theory. To deny a place for society in wellbeing would be unscientific. Consider the following quote from Morf and Mischel (2012, p. 33):

The self-construction process is intrinsically rooted within, and dependent upon, interpersonal processes that unfold in the social world. These social interactional processes and 'situations' involve not only significant other individuals but also relevant social groups that ultimately become part of one's 'collective self'.

The coalescence of being admits a substantial emphasis on society and the collective, it is just that everything originates with the individual. Note though that it is possible to be a loner. For example, people with a dismissive attachment style whose low trust in others leads to an emphasis on independence have self-esteem as high as people with a secure attachment style and broad social networks.

There is within the coalescence of being a detailed explanation of how an autonomously acting individual can hold collectivist values and put a group before their individual needs. To wit, the individual autonomously prioritises the group, self-regulating their purely-egoistic desires to instead affirm the internalised values of the group and affirm them in a selfdetermined manner. As Ryan and Deci (2004, p. 452) explain:

\footnotetext{
mathematically at least. Things are complicated by the fact that a culture seems to lose its ability to enculturate someone with values when it is not being adhered to by everyone around that person. This is one of the greatest challenges faced by secular, liberal, globalised, contemporary society. People are more and more allowed to find their own piece of heaven, but this slice of heaven is less and less palpable.
} 
Self-determination can be used to connote independent choices, but it can also describe acts of volitionally consenting to inputs such as obligations, inducements, urges, pressures or rising desires. As an example, consider a man who has fully assimilated and embraced collectivist cultural norms and practices. In a moment when he is pressured or tempted to act individualistically, he is likely to either implicitly or explicitly experience discrepancy and conflict. To be autonomous, he would have to find a meaningful way to coordinate the individualistic aim with his prior beliefs or revise either the aim or the prior beliefs. Anything less would represent less than full endorsement by the self and lack of integrity in behaviour.

Whether behaviour is autonomous or heteronomous has little to do with whether it affirms individual or collective goals (these are the same after internalisation) but instead on whether there is an external or internal locus of control.

Two empirical studies lend support to this analysis. Iyengar and Lepper (1999), in a study that was framed as critical of SDT's conception of autonomy, examined the aspirations, motivations and achievements of American and Japanese university students. They found that in both groups, having goals imposed by others led to the lowest levels of intrinsic motivation and well-being. However, among the American students, decisions made personally resulted in the highest levels of intrinsic motivation, while those made on advice from trusted insiders ranked second. Among the Japanese, these positions were reversed. The key thing then is not collectivism or individualism, but whether the individual is controlled or self-determined. People can feel more autonomous when endorsing and enacting the values of people with whom they identify but they will only achieve autonomy if it is they who choose the values. If they are forced to follow collective values under duress or manipulation, then ill-being will eventuate.

Further support comes from Devine et al (2008). Their study tracks households throughout Bangladesh. Both qualitative and quantitative survey techniques reveal that even in this highly collectivist society, and even amongst members who are discouraged or limited in their autonomy, such as women, issues of autonomy remain salient. Individuals expressed the desire to be consulted, to be financially independent, to have outside options thanks to a good education and simply "to be free". Importantly though, many people expressed their autonomy in terms of their relationships, not just with family members, but also with kin 
networks more broadly, and within community-based and development organisations. This is in line with the theoretical postulate that values might be collective but the processes underlying psychological well-being depend on autonomy and intrinsic motivation.

Another aspect of this study that is worth elaborating on is its coverage of the development of the Shammo organisation (see Devine 2002 for a more direct study). This was a community body instigated by development and other aid workers to organise collective action by locals against an oppressive system of land ownership that locked them into client-patron relationships with local landlords. Prior to the organisation emerging as a vehicle for collective action the villagers barely expressed a value for a more just distribution of local resources. Over time however, Shammo provided a source of sophisticated values that the locals internalised and eventually came to identify with. The organisation also served as an increasingly powerful platform for collective political action and allowed the villagers to contest their values against those of the local land-owners. They were ultimately successful in this endeavour. Shammo is obviously a story of power and institutions, but in the background is a neat narrative that captures the earlier analysis of the role of the collective, relatedness and internalisation in helping people define and affirm their values to achieve well-being.

In sum, the coalescence of being nourishes the need for relatedness by pushing the individual out into the world to affirm their values. This will inevitably see them gravitate towards those with similar values, internalise collective values and behaviours, engage in collective action with and against groups and promote their own interests through their group allegiances.

What about the other elements of eudemonia, hedonia and despair? Well...

Autonomy is taken care of by the actual self directing the individual's initial engagements in the world, and by the process of internalisation bringing initially extrinsic behaviours into the core self over time. The end result is a life in which most values are held and behaviours engaged in for intrinsic reasons.

The coalescence of being involves a harmonisation of the different elements of the "relation" that is our self over time. This process requires the elimination of dissonance and compartmentalisation, and the integration of identified but external values and behaviours with intrinsic ones. You can only do this by articulating the reasons why you value and do the things you do and the evidence these reasons are based on. What emerges from this process is an increasingly broad, deep, refined and sophisticated self-concept, one that you are very aware of because of all the time you have spent articulating its nature to yourself. This 
coalescence of being does not arrive at a final destination and so cannot totally short-circuit despair. But it does give the individual a clearer understanding of who they are and a measure of control over their destiny. This is the "being" that the French existentialists said we want. In order to maintain our sense of self over time we need to act with integrity. Abandoning one's values invites terror and dissolves identity. As such, there is a strong incentive to be consistent in behaviours and values over time, including one's ethical values and behaviours. This provides seriousness.

Note that in SDT the authority of ethics is placed firmly on the individual. It is the individual who administers the contingent factor-appeasing the ought self - that provides the initial introjected motivation to be ethical against one's crude self-interest. Deci and Ryan (2004, p. 248) explain that: "introjected regulation represents a prime instance of behaviour that is motivated by processes internal to the person but relatively external to the self'. What this means in the context of subjective ethics is that the ought-self involves self-regulation rather than self-determination. This is extremely relevant for ethical philosophy, which has historically struggled to explain ethical conduct because of an unnecessarily strict cleavage between selfish and altruistic behaviours. SDT's differentiation between intrinsically motivated and extrinsically regulated behaviours provides a way to bring other-regarding ethical behaviours into a self-interested analytical framework. It provides an explanation for the motivation system that lies behind individual humans' capacity to be self-interested and ethical simultaneously. SDT also explains how such regulated behaviours can gradually become intrinsically motivated through internalisation. Certain ethical acts and values may be difficult at first, but provided we identify with them they will gradually become habituated and eventually almost instinctive.

The prerequisite of integrity for coalescence reduces the severity of anguish by introducing a degree of compulsion (what Nietzsche called one's own necessity) into decision making. Anguish is also reduced by the steady accretion of a clear sense of self, because this sense of self provides guidance in the moment of decision making. It is further reduced by the gradual transition of a great deal of decision making to the level of automatised, instinctive behaviour. Anguish is inextricably tied to conscious processing. When decisions can be made by system 1 , they no longer provoke anguish.

Meaning in the coalescence of being comes from intrinsic motivation, flow and zest. Intrinsically motivated behaviours are enjoyable for their own sake, and intrinsic values 
emerge from our core self. Such values and activities are not transcendentally valuable - they are not written into the firmament. But they are valuable to $u s$, and this is enough to suffuse life with meaning and give us purpose. We matter if we choose to. In any case, we are distracted from any nihilistic thoughts by the omnipresence of flow. As we harmonise our actual and ideal selves the level of challenge inherent in our ideal self comes to be calibrated to the level of skill inherent in our actual self until the two are aligned. This provides most of the basis for flow. Internalisation provides an additional ingredient by transforming our behaviours and values into intrinsically motivated ones. The last ingredient is provided by mindfulness, which ensures that we attain high-quality feedback from our life at all times. With the conditions for flow met across a range of life domains, we increasingly step into "the zone" regardless of what we are doing. We develop a zest for life that dissolves any nausea.

The extension of flow across our life domains also nurtures our need for competence. Our goals becoming sufficiently challenging that when we meet them we feel a sense of achievement. But they are also calibrated to our skill level such that we are frequently successful and consequently have this sense of achievement on a regular basis. The coalescence of being is essentially a process of meeting the goal that is your ideal self, and so success at the coalescence of being necessarily gives rise to a feeling of competence.

Finally, coming back to hedonia, we have happiness. How does the coalescence of being bring about positive emotion? This analysis will be split into two parts. The first deals with direct causal chains between coalescence and positive emotion. The second discusses the integrated nature of affective and personality systems.

The coalescence of being is an enjoyable process because the attainment of our ideal self is an exhilarating experience. Goals are affectively coded, and goal achievement is consequently satisfying, even if this satisfaction fades and the goal needs to be replaced by a new one (Silvia and Eddington 2012). As the coalescence of being reaches completion, most of the things you do will provoke flow experiences, which are enjoyable. You will also correspond closely to your ideal self and this fact will be frequently disclosed to you, provoking positive emotions via feelings of self-worth. However, this positive emotion that accompanies the coalescence of being is meaningfully distinct from a high background level of positive affect. Goal achievement inevitably involves effort. The coalescence of being is full of suffering for your identity. But this effort and suffering is fundamental to flow, which 
cannot be achieved without the presence of high challenge. Life is thus punctuated by ups and downs, but you have a measure of control over the downs because you go through them willingly for the ups as you pursue competency and self-actualisation.

Happiness and self-actualisation are bound up together in other, more complex ways. Personality systems integration theory emphasises that affective signals are critical for coordinating shifts between self-maintenance and self-regulation (intuitive and conscious processing) as circumstances demand (Kuhl and Koole 2004, p. 420). Simplistically, if automatic decision making is leading the individual into unhealthy behaviours that reduce wellbeing they will receive negative affective signals. These prompt a shift from selfmaintenance to self-regulation so that conscious processing can be engaged to determine what is unhealthy about present behaviours. Once this mystery is solved, healthier behaviours can be engaged. As wellbeing rises from these, positive affect will result, and this signals to the personality system that it can automatise these behaviours and shift to self-maintenance. This process is assisted by the fact that affect is more intense when you are self-focused (Carver 2012, p. 53).

A more coalesced being — high self-concept clarity in psychological parlance — is also associated with greater resilience to negative mood. Higher self-concept clarity has been linked to less negative mood among college students under conditions of high life stress (Cohen et al 1997; Linville 1987; Dixon and 1991). Research also suggests that individuals with low self-concept clarity experience greater volatility in their affective state (McConnell et al 2009). Finally, as Showers and Zeigler (2012, p. 113) note, 'Low self-concept clarity is associated with neuroticism, low agreeableness, low self-esteem, low internal self-awareness, chronic self-analysis and a ruminative form of self-focused attention'.

It seems then that the coalescence of being and elements of eudemonic wellbeing are fundamental to hedonic wellbeing. You will struggle to be happy if you do not address the deep determinants of happiness implicit in the coalescence of being. These determinants, like the values inherent in your actual, ideal and ought selves, are all subjective. They are unique to you. It seems unsurprising in this context that techniques of mood management delivered in groups and thus inherently divorced from eudemonia have small effect sizes. Reflection and contemplation of oneself at a level deeper than one's mood is critical to the attainment of SWB, both in terms of eudemonia and hedonia. The Greeks were on the money when they emphasised "know thyself". Critics might reply to this point that such self-investigation may 
lead to rumination, which is unequivocally unhealthy (Papageorgiou and Wells 2004). Yet psychologists have developed scales for differentiating between rumination and reflection, and a skilled therapist should have little trouble explaining the difference to a patient (Trapnell and Campbell 1999).

A final point to take away from this discussion is that if the analysis in this chapter is correct, then life satisfaction scales are a questionable instrument for measuring dynamic changes in SWB because rescaling is frequent and profound. The iterative process of conceiving an ideal self and affirming it in action and social appraisal inevitably involves rescaling after successes and failures. Success provokes greater ambition, shifting the scales up, and failure provokes a reassessment of the individual's self-concept and goals, which changes the qualitative meaning of each point on that person's scales. Scales remain at least theoretically valid for measuring emotional state, but not long term existential satisfaction. I discuss these issues in greater detail in the next chapter, which concerns how SWB can be measured.

\section{Conclusion}

The coalescence of being is a model of the process by which the outcome of SWB as described in the wellbeing production function is attained. Its central mechanism is the harmonisation of the actual, ideal and ought selves over time through the setting of goals and their achievement or recalibration. Succeeding in goal pursuit brings a sense of competence. Calibrating our goals to our skill level over time increases the frequency of flow experiences. Acting with integrity towards our ideal and ought selves brings meaning and seriousness. In our acts and in the eyes of others we disclose our actual selves and this helps us to understand who we are, bringing identity and annulling anguish. The automatisation of behaviours over time as we become more comfortable with ourselves, goals and values dampens anguish further by reducing the frequency with which we are confronted by the need to consciously choose. The coalescence of being is most effective when we pursue intrinsically motivated goals that are self-concordant. This ensures autonomy. It also brings us into collectives with like-minded individuals, nourishing our need for relatedness. This whole process is punctuated and guided by affective signals. It is through our emotional responses to success, failure and new endeavours that we come to understand what goals and values are right for us. However, we struggle to improve our SWB simply by treating these affective signals-we 
must understand their association with the deep determinants of our SWB in the eudaimonia and despair dimensions.

In the next, final chapter, I investigate how we can measure SWB as described by the production function and the coalescence of being. I pay particular attention to SWB measurement in the context of economics and public policy. The motivating challenge of the next chapter is that while each individual aspect of the SWB model described in this thesis is supported by empirical evidence, the model is not perfectly borne out by contemporary research into global SWB evaluations using life satisfaction scales. Succinctly, there seems to be a limit to how satisfied people can get, and powerful forces drawing them back to set-point levels of satisfaction over time. I argue that these results are perhaps at least partially artefactual in nature - they are a function of the measurement instrument rather than a real phenomenon. People recalibrate their scales over time, which obscures the fact that are actually growing in life satisfaction in absolute terms. 


\section{Chapter 10}

\section{Measuring Subjective Wellbeing}

If you cannot measure it, you cannot improve it.

- William Thomson, Lord Kelvin

\section{Introduction}

This chapter analyses the different ways subjective wellbeing has been measured for applications in economics and public policy and considers innovative alternatives. It makes three principle points. The first is that measuring SWB in the context of economics and policy brings with it unique challenges. From a practical perspective, SWB measures must be relatively cheap and quick to administer because otherwise they are hard to incorporate into the large-sample social surveys that are the workhorses of policy analysis. For many applications in policy and especially in economics, SWB measures must also produce measurements of individual SWB that are at least ordinally comparable interpersonally. This requirement is most acute in welfare analysis.

The second principle point of the chapter is that the primary metric of SWB research to date, namely life satisfaction scales, is a problematic empirical instrument, especially for analysing dynamic trends and causal changes in SWB. This is because of issues pertaining to scalenorming, which is where the amount of absolute satisfaction associated with each point on a respondent's scale changes over time. Scale-norming makes responses difficult to compare interpersonally or inter-temporally. This chapter presents empirical evidence that scalenorming is more widespread that contemporary research in SW-B might believe. 
This brings us to the third principle claim of the chapter, which is that the evidence for scalenorming is strong enough that SWB as a field should at least consider experimenting with alternative metrics to scales. Two such metrics are presented and discussed: plots and worms. In addition to developing new quantitative metrics, SWB research in policy also needs to develop more sophisticated metrics for capturing values and how they are learnt. Taking averages over very large samples is fast running out of usefulness because, as the previous chapters have argued, values are critical to wellbeing and everyone has different values.

\section{Ways of measuring subjective well-being}

There are many ways to measure SWB. One finds the following metrics in relatively common use:

1. Experience sampling methods (discussed in chapter 6)

2. Functional magnetic resonance imaging (colloquially known as brain scans)

3. Affect measures, such as the number of times you smiled yesterday

4. Inventories, such as Beck's Depression Inventory (Beck et al 1961). These are short surveys that employ Lickert-scale ${ }^{25}$ questions to measure a construct

5. Lengthy surveys using factor loading and Lickert scale methods to assess someone's SWB. A prominent example is Ryff's (1989b) measure of psychological well-being.

6. Life satisfaction scales (and satisfaction scales more generally, such as job satisfaction and financial satisfaction).

A key limitation of methods 1-4 is that they are not global measures of SWB, meaning that they capture only one dimension of wellbeing (typically hedonia), or even just one aspect, like affect. The limitation of method 5 is that it is time consuming and thus relatively expensive to administer. This is not a major weakness in many settings, such as the derivation of the psychological theories that were used to inform the theory of SWB developed in chapters 4-9. However, it is a much greater weakness in the context of public policy and most applications in welfare economics. This is because research for such applications typically takes a nation or at least a large community as its scope and is interested in complex social phenomena and interactions. This research thus requires a large empirical sample (such

\footnotetext{
${ }^{25}$ Lickert-scales involve questions phrased in the form of, for example, "to what extent do you agree with the following statement..." and then options ranging from, for example, strongly agree to strongly disagree (Lickert 1932). The data produced by these questions can be analysed using factor-loading techniques.
} 
as the tens of thousands used in national longitudinal social surveys), and the cost of administering metrics in the context of such samples grows exponentially as the complexity of the metric increases. Sometimes smaller representative samples can be used, but here researchers must worry about external validity. That is, the findings of the research must be generalisable to other contexts. If the sample is not large then external validity typically necessitates rich surveys that provide a lot of data to use in the construction of control variables for use in statistical research, and multiple survey waves so that fixed effects and difference-in-difference methods can be brought to bear. The cost of administration again becomes a concern and parsimony a virtue.

Life satisfaction scales are relatively global measures of wellbeing and are quick and cheap to administer (Diener et al 2013). It is unsurprising then that they are the workhorse metric in happiness economics and policy research exploring SWB (see for example: Clark, Frijters and Shields 2008, Clark et al 2018, Dolan et al 2008, Graham 2017, Helliwell, Layard and Sachs 2017, Stephenson and Wolfers 2013, Van Praag and Ferrer-i-Carbonell 2004). A typical life satisfaction scale question is the following, from the Household Income and Labour Dynamics of Australia Longitudinal Panel (HILDA): "All things considered, how satisfied are you with your life at this time on a scale from 1-10?" Such questions feature in just about every major longitudinal social survey. Sometimes Cantril's ladder of life is used instead, such as in the World Values Survey. This question involves presenting respondents with the image of a ladder, typically with 10 steps. Respondents are then asked something like the following:

Assume that this ladder is a way of picturing your life. The top of the ladder represents the best possible life for you. The bottom rung of the ladder represents the worst possible life for you. Indicate where on the ladder you feel you personally stand right now.

The major difference between conventional life satisfaction scale questions and the ladder of life question is the inclusion of the phrases "best possible" and "worst possible". It is hoped that these phrases will anchor respondents' scales to some fixed points over time.

Many of the findings of SW-B research using scale questions are striking. Two in particular stand out, at least to economists. The first is adaptation: the claim that people rapidly acclimatise to shocks to their life satisfaction, even major ones like spinal injury, and return to a long-run set point over time, typically within 2 years (Clark et al 2008, Dyrdal and Lucas 
2013, Lucas et al 2003, Oswald and Powdthavee 2008, Powdthavee and Stutzer 2014, Specht et al 2011). This is the "strong adaptation" hypothesis, and it is reaching the status of accepted wisdom in SWB circles. This is despite strong adaptation running counter to some theories, notably the coalescence of being, which suggests that people can achieve sustained increases in their subjective well-being over time. The second striking finding is that sustained periods of economic growth do not appear to lead to sustained increases in wellbeing, contrary to claims by economists (Deaton 2013). As discussed in chapter 6, the most recent source of this claim is research from Easterlin et al (2017) suggesting that China's meteoric economic growth over the period 1990-2015 did not seem to translate to increases in life satisfaction. Layard (2005), among others, posited that the results in happiness economics can be explained by the effects of the hedonic treadmill and changes in reference points as people get wealthier.

More mainstream economists have responded that strong adaptation is perhaps at least partially an artefact of the data-generating process rather than a real phenomenon. Rather than adapting to shocks, perhaps people scale-norm (Frederick and Lowenstein 1999). "Scalenorming" (sometimes referred to as scale-recalibration or rescaling) is where the qualitative meaning of the points on a person's scale change over time such that, for example, an 8/10 in wave 1 of a survey corresponds to a different absolute level of satisfaction than an 8/10 in wave 3. If scale-norming takes place, then the scale a person uses to answer scale questions might be intended to represent increasing levels of life satisfaction even if the score they give on that scale does not change. This would explain Easterlin's result and many others in happiness economics - people's responses are not changing but their scale is, we just can't observe the latter. In the next section I give a more thorough account of the difference between adaptation and scale norming. I then present theoretical arguments and empirical evidence that attest to the existence of scale-norming.

\section{Adaptation and scale-norming}

Below is a graphical depiction of adaptation taken from Inferna and Wiest's (2018) study of the impact of disability on life satisfaction over time (figure 1). It shows individuals experiencing negative shocks to their life satisfaction from disability, but quickly returning to their long run "set point" (Lucas et al 2004). Inferna and Wiest's result echoes that of Brickman et al (1978), who famously found that lottery winners 
and sufferers of spinal cord injuries adapted to their respective positive and negative life satisfaction shocks within two years, returning to their earlier satisfaction levels. They were the first to posit the existence of the hedonic treadmill. The possible role of changing reference points was posited later (Kahneman 1999, Layard 2005).

\section{Figure 1: Adaptation to Disability}

(B)

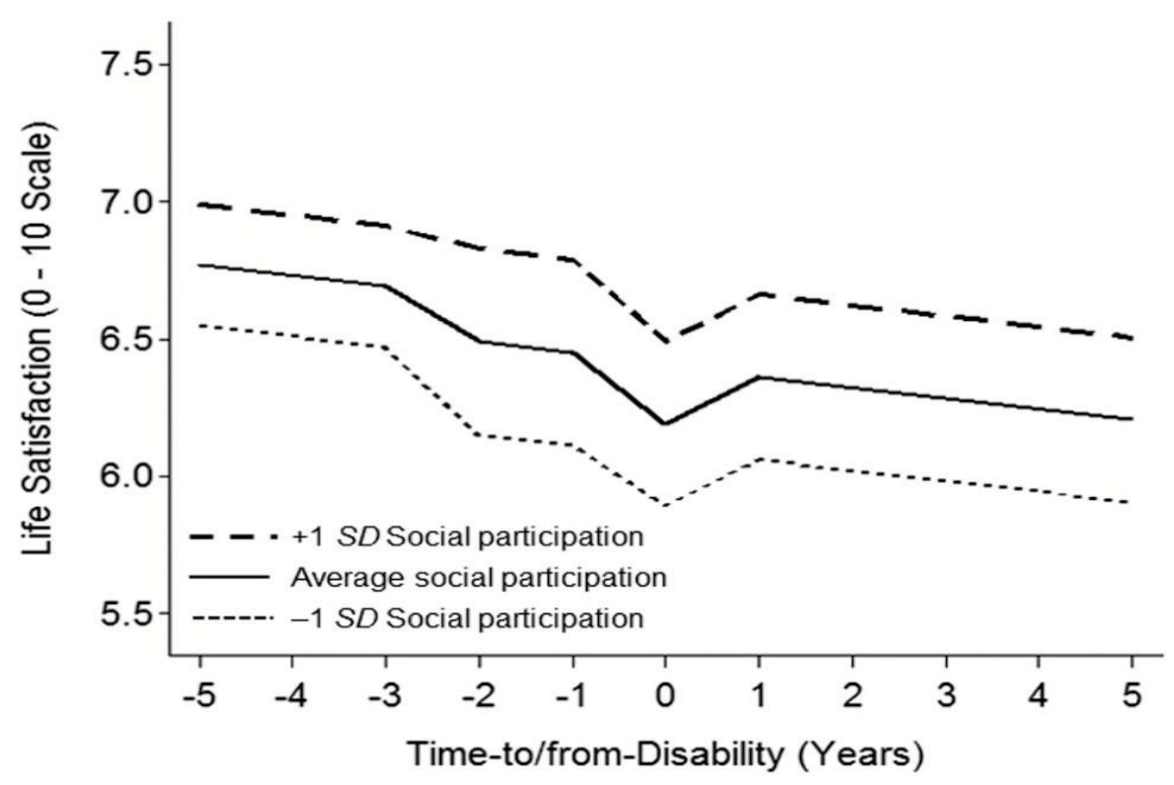

Brickman, Coates and Bulman's research findings spurred an extensive research effort that has grown in recent decades as data availability has increased. As detailed in chapter 6, similar adaptation results have been found for a range of life events including marriage, divorce, income growth, retirement, moving out of home and migration (Yap et al 2014). Some authors have argued that adaptation is a fundamental tendency of human psychology (Cummins 2014). A recent volume summarising research into adaptation questioned whether it is at all possible to permanently alter people's satisfaction levels (Sheldon and Lucas 2014). While the editors ultimately concluded that some events, like prolonged unemployed, the death of a spouse and cosmetic surgery, did in fact have the potential to permanently alter an individual's satisfaction set point they did not question the ubiquity of adaptation. This is indicative of the general acceptance in the field of strong adaptation as characteristic of life satisfaction dynamics. 
Subjective well-being scholars believe in adaptation because it is "what we see in the data". No other interpretation makes sense. However, adaptation is both an interpretation of "what we see in the data" and of the data-generating process. Adaptation assumes that the data-generating process produces a relatively accurate account of an individual's life satisfaction that can be at least ordinally compared intrapersonally over time. The scale-norming interpretation, by comparison, is skeptical that the data generating process in scale questions produces such comparable responses. Where adaptation emphasis cognitive phenomena, scale-norming emphasises measurement error.

Scale-norming is where the qualitative meaning of the points on a person's scale change over time. The nature and implications of scale norming for life satisfaction measurement is captured well in the following comments by Economist Martin Ravallion. He was replying to Easterlin's (2017) study of life satisfaction in China (quoted in Clark and Senik 2014, p. 246):

Economic development is a process of structural change, which changes people's reference groups and scales. It changes how you think of the world where you live when you move from a village, where the reference group is very narrow, to a city with a very vast set of people at different levels of living. In that process, the scale of subjective well-being that we use is surely going to change.

Ravallion is here implying that it is wrong to conclude from the data Easterlin uses that people's life satisfaction has not improved because while their scale response hasn't changed over time, their scales must have. Consequently, while they are reporting similar numerical values for life satisfaction over time, these values represent growing absolute amounts of satisfaction.

Below is a graphical depiction of scale norming (figure 2). The y-axis tracks absolute life satisfaction on a continuous scale from 0 to infinity. The $x$-axis tracks years of an annual survey incorporating a life satisfaction scale question. The graph plots an individual's responses to these annual questions both in terms of their absolute life satisfaction and their year-on-year scale response. In this hypothetical scenario, the individual's absolute life satisfaction improves over the four years from 7 to 15 . However, their scale response stays the same at 7 in wave 1 and 7 in wave 5. The reason for this discrepancy is that the individual changes the scale they use to make 
their scale response each year starting from wave 3 as their life satisfaction improves. In wave 3 , their life satisfaction improves to $10 / 10$ on their original scale. However, they can still see further potential improvements in their life satisfaction so they don't want to say they are $10 / 10$. Unable to effectively communicate this improvement in their satisfaction on their existing scale, the individual's only option is to use a new scale. The surveyor does not observe this change. As such, they may wrongfully conclude that this person's life is not improving when in fact their absolute life satisfaction has more than doubled by the end of the study period.

\section{Figure 2: Scale Norming}

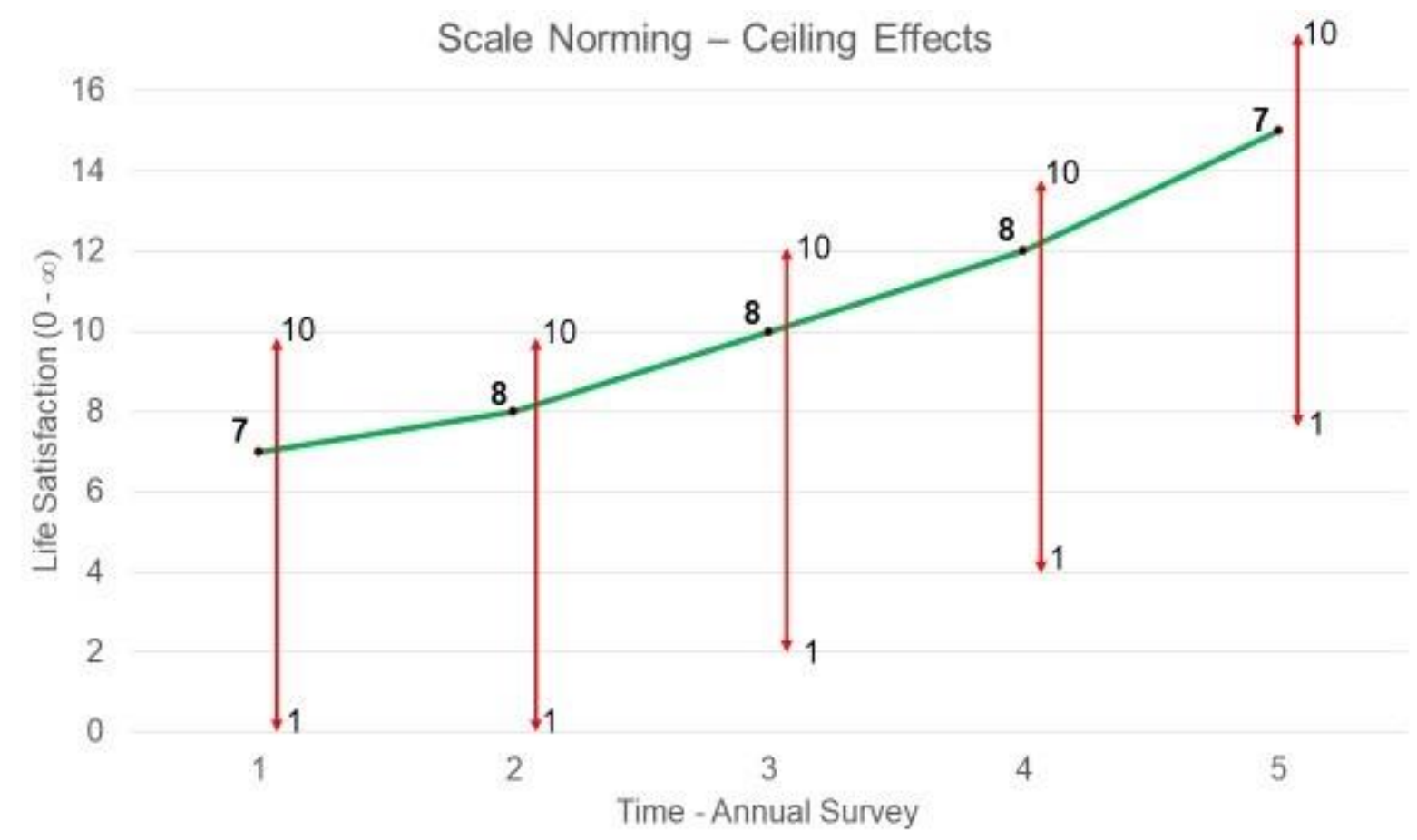

It is worth noting that figure 2 depicts ceiling effects, which are a specific driver of scalenorming. Ceiling effects in this context refers to a situation where an individual cannot effectively communicate improvements in their life satisfaction on a scale question because they already reported a very high satisfaction in the previous wave of the survey. Ceiling effects might be implicated in a range of findings in the life satisfaction literature, such as why economic growth has a less noticeable impact on subjective well-being than economic downturns (De Neve et al 2018). During growth periods, improvements are buried in scale norming and don't show up as improving scale responses. In contrast, there is plenty of room to move down in the scale and so recessions show relatively strong effects. Ceiling effects are 
an important potential driver of scale-norming, but scale-norming is a more general phenomenon. Chinese migrant workers and spinal injury patients, for example, would struggle to maintain meaningfully similar scales across survey waves pre- and post-shock because of the dramatic qualitative changes in their lives rather than because of ceiling effects.

\section{Theoretical arguments for scale-norming}

Life satisfaction scale questions are supposed to measure evaluated wellbeing as distinct from experienced wellbeing. Pavot and Diener (1991) describe the process of making an evaluation of this sort as involving the individual constructing a "standard" that they perceive as appropriate for themselves, and then comparing the circumstances of their life to that standard. Fleurbaey and Blanchet (2013) developed a model of "subjective well-being as it can be retrieved with typical questionnaires" that neatly illustrates three separate but related problems that are likely to plague such evaluation exercises: the scope, ranking and calibration problems.

Fleurbaey and Blanchet's model takes the following form. They begin with a vector $l_{i}$ that covers "the diversity of states, activities and possibilities enjoyed or endured by an individual [i] over the course of their life" (p. 175). When someone answers a life satisfaction questionnaire, they consider $l_{i}$ and there is some function $\xi$ i that maps this vector into possible answers to the scale question. This is similar to the life satisfaction "reporting function" discussed by Oswald (2008). The individual's answer to a life satisfaction question is thus not $l_{i}$ but instead $\xi \mathrm{i}\left(l_{i}\right)$. Fleurbaey and Blanchet explain that:

$\xi \mathrm{i}\left(l_{i}\right)$ must lie in a given scale, which can be a verbal scale (e.g. very satisfied/fairly satisfied/not very satisfied/not at all satisfied), or a numerical scale (e.g. from 0 to 10). The cognitive problem for the individual is to put the many dimensions of $l_{i}$ into one of a few ordered categories.

The scope problem concerns what aspects of $l_{i}$ are relevant for the individual to consider. For example, what time frame is appropriate - today, this week, the time since the last survey? Should the state of the household be considered or just the individual? What about the state of the world in general? 
The ranking problem refers to the cognitive difficulty of arranging relevant possible lives, including the individual's actual life, $l_{i} *$, into an ordinal pattern. The complexity of this exercise may induce respondents to focus on salient aspects of their immediate situation and forget other relevant dimensions of their life. A famous example of this is the "focusing illusion" (Schkade and Kahneman 1998). Respondents may focus on, say, the poor weather in their area and fail to take into consideration relatively low local living costs.

The calibration problem is about how the individual translates the position of $l_{i} *$ in their own ranking to a category in the life satisfaction scale questionnaire. There is a strong framing effect present that arises out of the fact that the scale offered is closed. This is in contrast to real life, where many considerations relevant to life satisfaction are open, like income, or have fuzzy limits, like pain or longevity. The closed scale forces respondents "to move from reasoning in terms of life content to a reasoning in terms of a statistical distribution" (Fleurbaey and Blanchet 2013, p. 181). The calibration problem is which distribution to choose. For example, should the respondent choose from among the lives available to all humans, including Jeff Bezos, or rather from among those that seem realistically possible for themselves? Different calibrations across respondents could reasonably be expected to introduce meaningful noise into the data. Indeed, Bond and Lang (forthcoming) provide empirical evidence to suggest that many headline making findings of subjective wellbeing research, like the Easterlin paradox and u-shaped relationship between age and life satisfaction, are not robust to different adjustments for the calibration problem.

To summarise, the problem with scales is that we want to know about $l_{i}$ but we can only ever observe $\xi \mathrm{i}\left(l_{i} *\right)$. Furthermore, both $l_{i}^{*}$ and $\xi \mathrm{i}\left(l_{i}\right)$ could change over time such that responses to life satisfaction scale questions become neither inter-temporally nor inter-personally comparable, even ordinally. This may go some way to explaining why scale responses have low test-retest coefficients of $0.5-0.7$ over even short periods of 1 day to 2 weeks (Krueger and Schkade 2008)

The coalescence of being suggests that $l_{i} *$ and $\xi \mathrm{i}\left(l_{i}\right)$ should in fact be expected to change over time. Coalescence suggests that people make adjustments to their values and perceptions of their life following success and failure in achieving goals and other sources of information about the self. Many of these episodes could be significant enough to trigger scale-norming. For example, an individual expecting to be accepted to a top graduate school would possess this expectation because they believe their "actual self" is the kind of individual who gets 
accepted to such schools. Their ideal self - their "best possible life"-is conceived in part on the basis of this assessment. If they do not receive an acceptance letter then we would expect their life satisfaction to decline, but we would also expect the qualitative meaning of the points on their scale to change in unpredictable ways. This is because the individual must reassess their actual self and their ideal self on the basis of the evidence inherent in not being accepted to the good graduate schools. Perhaps they will completely abandon their academic ambitions and instead become a tennis coach. At that point, their "best possible life" will no longer be associated with a professorship, but with owning a tennis club or coaching on the pro tour. Such recalibration could occur after receiving acceptance letters as well. In that case, the individual would adjust their long term plans and ambitions on the basis of the trajectory that they are now confirmed to be on. The dissolution of ambiguity concerning the self can easily change what we think is "possible" for us.

\section{Empirical evidence for scale-norming}

It's all good and well to posit theoretical reasons why life satisfaction scales might be hopelessly contaminated, but if these theories don't turn out to be so problematic empirically then we can proceed. This is typically the rejoinder of SW-B scholars to criticisms like those of Fleurbaey and Blanchet. They point to the extensive psychometric validation exercises that have been conducted on scale metrics (Diener, Inglehart and Tay 2013), and they advise caution and use caveats. For example, the OECD guidelines of measuring SWB conclude (2017, p. 12):

Although subject to some methodological limitations, it is clear that for many potential uses, measures of subjective well-being, when carefully collected, are able to meet the basic standard of "fitness for purpose".

Advocates of life satisfaction scales also point to empirical results that suggest that while scales are not precision instruments, the degree of imprecision they suffer is not fatal. For example, Ferrer-i-Carbonell and Frijters (2004) found that assuming ordinal or cardinal comparability between scale responses makes little difference to regression coefficients.

What is needed is thus empirical scrutiny of the extent and severity of scale-norming. There are some relevant studies from the quality of life literature on response shift (Daltroy 1999, Kapteyn et al 2013, Schwartz et al 2006, Lacey et al 2008, Ubel et al 2005). However, these 
are typically studies of ill health where ceiling effects are not at play. These studies are sometimes also clouded by an inability to differentiate between adaptation and scale-norming (Ubel et al 2010).

One strong piece of evidence regarding scale-norming that arguably does not suffer from these shortcomings is Stillman et al's (2015) study of Tongan migrants to New Zealand. New Zealand uses a lottery system to allocate visas to would-be migrants from Tonga, which allows for pseudo-experimental methods to be used to identify the causal effects of migration. Prior to the lottery, all applicants are asked about their life satisfaction, and the average is 8/10. Applicants who are successful in the lottery migrate to New Zealand and typically experience large increases in their real incomes, which we would expect to increase their life satisfaction. Yet 2-5 years after the lottery, both successful and unsuccessful applicants are interviewed again, and the average life satisfaction of both groups is $8 / 10$. The income growth seems to have had no effect. This lends support to the adaptation hypothesis. However, in the second wave of their survey Stillman et al asked both groups how they felt prior to the lottery (this is equivalent to a "then test"- see Schwarz et al 2006). The unsuccessful applicants said they were 8/10, which is what they said at the time. The migrants said that they were $6 / 10$. It appears that the shock of migration has provoked a transformation in the qualitative meaning of the migrants' scales: they have scale-normed.

Stillman et al's finding calls into question the comparability of scale responses. The migrants' $8 / 10$ in wave 2 is meaningfully higher than their $8 / 10$ in wave 1 , which suggests that these responses are not inter-temporally comparable. Their 8/10 in wave 2 appears to be higher than the $8 / 10$ of the unsuccessful applicants in wave 2, which suggests that these responses are not inter-personally comparable. Stillman et al's result lends credence to the existence of scale-norming and ceiling effects, and speaks to the severity of the scope, ranking and calibration problems. ${ }^{26}$ It is a challenge to a large body of existing research on the dynamics of life satisfaction over time and demands follow up studies.

\footnotetext{
${ }^{26}$ Stillman et al's result diverges from those found in other migration studies, such as those collated in the World Happiness Report 2018 (Helliwell et al 2018). These studies typically find large increases in life satisfaction as reported on scales following migration. The discrepancy between these results and that of Stillman et al could be explained by ceiling effects. Atypically, New Zealand employs a targeted migration system that only admits individuals who are, in a sense, already elite. This is why the applicants' average life satisfaction is 8/10. This is much higher than the migrants in the Happiness Report studies, who start from a lower base. The Tongan migrants are thus more likely to experience ceiling effects and scale-norm if their life satisfaction improves following migration to New Zealand.
} 
Investigating scale-norming in life satisfaction scale response data requires the development of alternate metrics that extend life satisfaction scales, like Stillman et al's retrospective question. While a burden, this should be seen as an opportunity because while life satisfaction scale questions have been quite extensively validated internally, they have not been validated much relative to other possible metrics. The need to explore such alternates is eloquently made by Alexandrova (2017, p. 133) in her A Philosophy for the Science of Wellbeing:

If a measure correlates in expected ways with suicide rates and self-harming, health, smiling, cortisol levels, and so on, this is evidence that this measure is plausible. But in addition to plausibility we need evidence that this measure is better than another plausible measure.

In the next section I present such an alternate measure, perform a preliminary assessment of its plausibility and present results from a study using it designed to show up scale-norming if it exists. I call this measure the life satisfaction plot.

\section{Life satisfaction plots}

Life satisfaction plots ask respondents to draw a line-graph of their life satisfaction over some time horizon. They access the same concept as life satisfaction scales but focus on long-term dynamics in life satisfaction rather than evaluations of the present. They ask respondents to reflect on their current situation relative to the past, in effect asking them to apply the same scale over some time horizon. This should make responses more robust to scale-norming issues than annually administered scale questions. Like scale questions, plots are quick and cheap to administer.

In the study documented here, the time frame was ten years and respondents were asked to draw their line graph in the following area (figure 3). A primary research question was to compare life satisfaction trends as depicted in life satisfaction plots with those constructed by stringing together responses to life satisfaction scale questions administered in annual longitudinal social surveys like HILDA. 


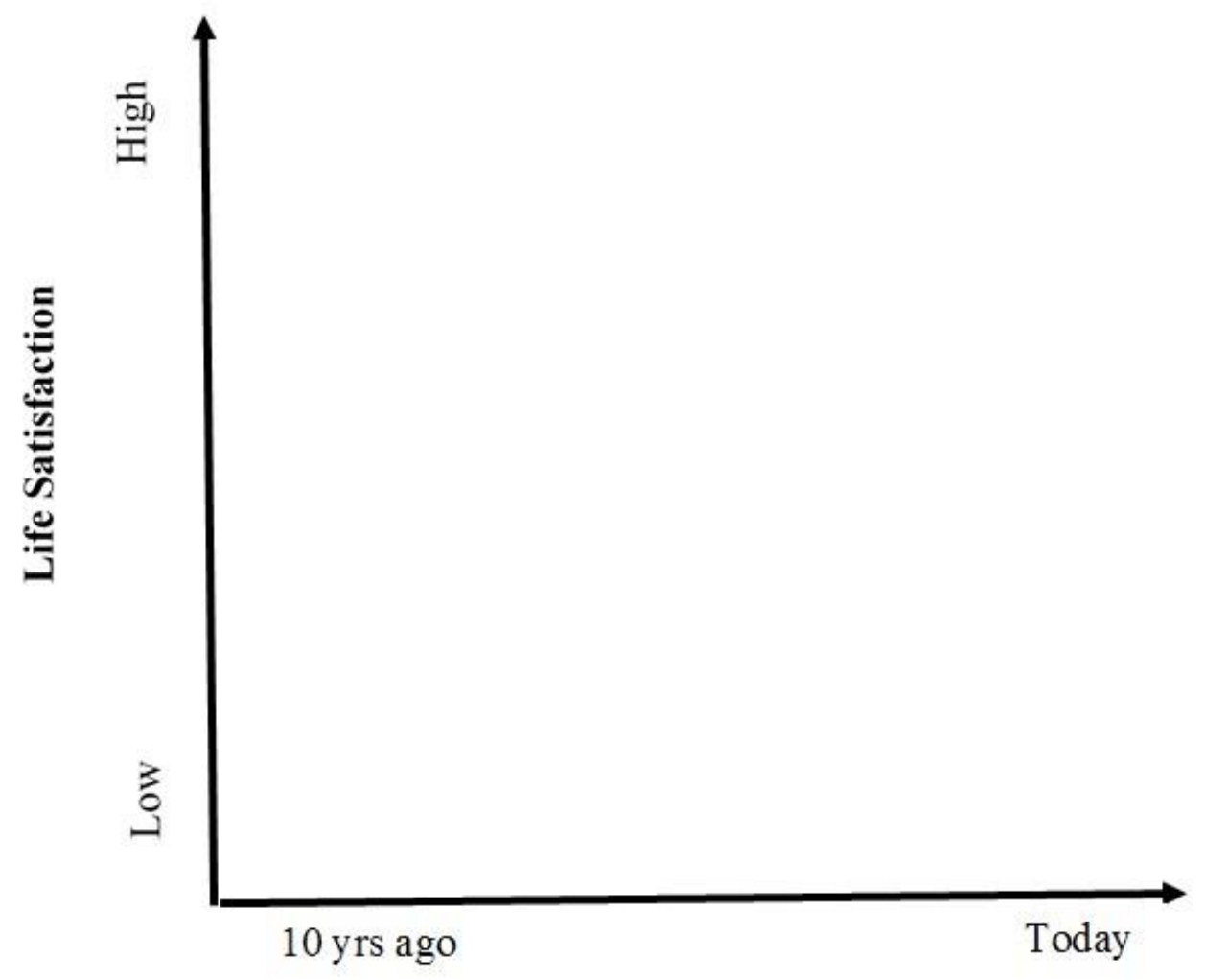

\section{Time}

To aid the investigation of scale-norming and adaptation in life satisfaction scale studies, the study documented here also included two other questions. The first was the standard life satisfaction scale question used in social surveys. The study used the HILDA wording, namely: "All things considered, how satisfied are you with your life at this time on a scale of 1-10?". This wording was chosen to aid comparability in responses, as the sample used for this study was Australian. The second question was a variation on the retrospective question used by Stillman et al, namely:

Think back to 10 years ago. Meditate for a moment on the activities you did, the concerns you had, the good and bad things that were in your life and how happy or sad you were. Place yourself back in your mind at the time.

10 years ago, what would you have answered to the following question: all things considered, how satisfied are you with your life on a scale of 1-10? 
Scale-norming can be investigated by comparing trends in life satisfaction plots with responses to the two scale questions. If respondents show significant change in one direction in their life satisfaction plots but no commensurate change in their scale response from time 1 $\left(t_{1)}\right.$ to $t_{10}$ this would suggest that they are scale-norming. A high scale response score for $t_{1}$ coupled with a sustained upward trend in their plot would add further credence to this inference as this would indicate ceiling effects kicking in. Adaptation can be easily observed in the plots by looking for convergence back to a baseline over time following shocks. The 10 -year time horizon provides enough scope for this adaptation to occur.

\section{Survey implementation}

The three-question survey was administered to 143 masters from 3 separate classes at the Crawford School of Public Policy at the Australian National University. The questions permuted for each class: class 1 (plot, satisfaction now, satisfaction then), class 2 (then, now, plot) and class 3 (now, then, plot). This is a purposeful rather than a representative sample. It has characteristics that make it ideal for identifying whether responses to plot questions are likely to differ in systematic ways from responses to scale questions. The majority of the Crawford School's students are successful career bureaucrats in their late 20s to early 30 s from middle class households studying on government scholarships. They have typically experienced sustained increases in their objective well-being over time starting from a high base. As such, they are liable to suffer from ceiling effects. This makes them an ideal sample for potentially falsifying both the scale-norming and adaptation hypotheses.

After the surveys are administered, a 10x10 grid is overlaid onto the plots drawn by respondents. This grid is used to derive life satisfaction scores from 1-10 (in increments of $0.5)$ for years 1-10. These can then be used to analyse the curvature of the plots and compare them to scale responses. Three further variables of interest are the net change in respondents' life satisfaction as depicted in their plots ("netchange"), the change in their life satisfaction as depicted in their scale responses ("change"), and the total volume of change in their plots ("Totalchange"). These three variables are calculated as follows:

$$
\begin{aligned}
\text { netchange }= & \left(t_{2}-t_{1}\right)+\left(t_{3}-t_{2}\right)+\left(t_{4}-t_{3}\right)+\left(t_{5}-t_{4}\right)+\left(t_{6}-t_{5}\right)+\left(t_{7}-t_{6}\right) \\
& +\left(t_{8}-t_{7}\right)+\left(t_{9}-t_{8}\right)+\left(t_{10}-t_{9}\right)
\end{aligned}
$$


Netchange could also be represented as $t_{10}-t_{1}$, but some respondents drew short plots and thus had missing values for some early and late time periods. The specification of netchange above overcomes this problem.

$$
\text { change }=\text { Life Satisfaction }_{\text {now }}-\text { Life Satisfaction } \text { then }_{\text {fif }}
$$

Where "now" corresponds to the life satisfaction scale question about present satisfaction, and "then" corresponds to the question about ten years ago.

$$
\begin{aligned}
\text { total change } & =\left|t_{2}-t_{1}\right|+\left|t_{3}-t_{2}\right|+\left|t_{4}-t_{3}\right|+\left|t_{5}-t_{4}\right|+\left|t_{6}-t_{5}\right|+\left|t_{7}-t_{6}\right| \\
& +\left|t_{8}-t_{7}\right|+\left|t_{9}-t_{8}\right|+\left|t_{10}-t_{9}\right|
\end{aligned}
$$

Total change is the sum of the absolute value of change in each period depicted in an individual's life satisfaction plot.

\section{Summary Statistics}

Table 1 below and figures A1-A5 in the appendix provide descriptive statistics for the major variables used in this study. Equivalent values from the first 10 years of the HILDA study are provided by way of comparison. To improve comparability, the HILDA figures are drawn using a sub sample restricted to individuals between the ages of 17 and 35 in the first 10 waves (from 2001-2011) who attain a postgraduate qualification by wave 10. This reduces the comparison group to 218 people.

\section{Table 1 - Summary statistics}

\begin{tabular}{|l|l|l|l|l|}
\hline \multicolumn{1}{|c|}{ VARIABLE } & \multicolumn{1}{|c|}{ MEAN } & \multicolumn{1}{|c|}{ S.D. } & \multicolumn{1}{|c|}{ HILDA } & \multicolumn{1}{c|}{$\begin{array}{c}\text { HILDA } \\
\text { (S.D) }\end{array}$} \\
\hline Total change & 5.8 & 4.5 & 4.2 & 3.4 \\
\hline Net change & 2.9 & 3.0 & N/A & N/A \\
\hline Change & 1.2 & 1.9 & -0.07 & 1.5 \\
\hline LSAT "Now" & 7.7 & 1.1 & 7.9 & 1.1 \\
\hline LSAT "Then" & 6.6 & 1.8 & 7.9 & 1.2 \\
\hline
\end{tabular}

All the values are higher in the plotting study than in HILDA. It seems that the plotting instrument picks up more dynamism in life satisfaction over time than life satisfaction scale 
questions administered annually. This is most obvious when comparing "change" in the plotting study with HILDA. The mean value in HILDA is very close to zero (which is also the median value), whereas in the plotting study the mean is 1.2 with a median of 1 . This result may reflect the fact that the plotting instrument asks respondents to explicitly consider the present relative to the past, unlike annual scale questions. The netchange variable has no equivalent in HILDA, but with a mean of 2.9 it suggests that most people are experiencing some change in their life satisfaction over time-something that is not reflected in the HILDA results.

The only noteworthy difference in reporting across the three classes was that class 2 reported both higher life satisfaction then and higher life satisfaction now: class 1 (then: 6.8, now: 7.4), class 2 (then: 7.5, now: 8.7) and class 3 (then: 6.4, now: 7.7). The obvious explanation for this is that they were asked about their satisfaction "then" before their satisfaction "now", whereas in the other 2 classes the question order was reversed. The respondents gave a relatively high response to their life satisfaction 10 years ago, but then in order to communicate that their lives had improved, they gave an even higher response for life satisfaction today. It appears that framing life satisfaction in relative terms exerts a strong effect on responses. However, what is consistent across frames is a desire to communicate improvements. We don't see such improvements in longitudinal panels (see results below).

\section{Consistency analysis}

Responses in the plotting study were broadly consistent and in line with intuition. The mean difference between the life satisfaction scale response for today and the plot response for time 10 was 0.078 with a standard deviation of 1.5 . This is not significantly different from zero. The mean difference between the life satisfaction scale response for ten years ago and the plot response for time 1 was 1.92 with a standard deviation of 2.3. This was significantly different from zero. However, this does not indicate inconsistency, as the plot will often track movement within scale responses rather than changes between scale responses. Indeed, this is implied by coefficients from a regression of change as a function of netchange and a constant. The beta coefficient on netchange is 0.36 , suggesting that three units of plot change are required to produce a single unit of scale change. The estimate is statistically significant at 
the 1 per cent level, and the model shows modest explanatory power with an $\mathrm{R}^{2}$ of 0.32 . The consistency of the relationship between the plot and scale responses is also evident from the figure A6 in the appendix, which depicts the change variable as a portion of the netchange variable. The relationship expressed in the regression coefficient is evident.

The correlations between variables is also encouraging. Netchange and change correlate at 0.6 , "life satisfaction then" correlates with plotted satisfaction in time 1 at 0.5 , and "life satisfaction now" correlates with plotted life satisfaction in time 10 at 0.5 .

\section{Results}

The plotting study had three primary research questions. The first is whether the dynamics of life satisfaction as measured by the plotting instrument differ meaningfully from those measured using life satisfaction scales. The second is whether scale norming is something we should be concerned about. The third is whether adaptation is as strong as appears to be the case when examining life satisfaction scale data. Results for each of these questions are reported below.

\section{Dynamics of life satisfaction in scale responses compared to plots}

The life satisfaction plots show markedly different trends and dynamics in life satisfaction over time than the life satisfaction scale responses in HILDA. On average across the whole sample (figure 5), life satisfaction improves by 3 full points from 4.6 to 7.6 over the 10 -year plot window. By comparison, life satisfaction in HILDA (figure 6) doesn't change at all over the first ten years of the panel for the comparison group. The only change over time in HILDA is two periods of very minor decline, from an average of 7.9 to 7.8 in waves 4 and 5 . The plotting study shows increases in every period. The plotting study would suggest life is getting better, while the year-on-year scale responses suggest no change. It would be interesting to see whether this result replicates in other famous cases of no improvement in life satisfaction scale responses over time, such as in China from 1990 to 2015.

Figure 5: Average life satisfaction per period in plotting study—whole sample 


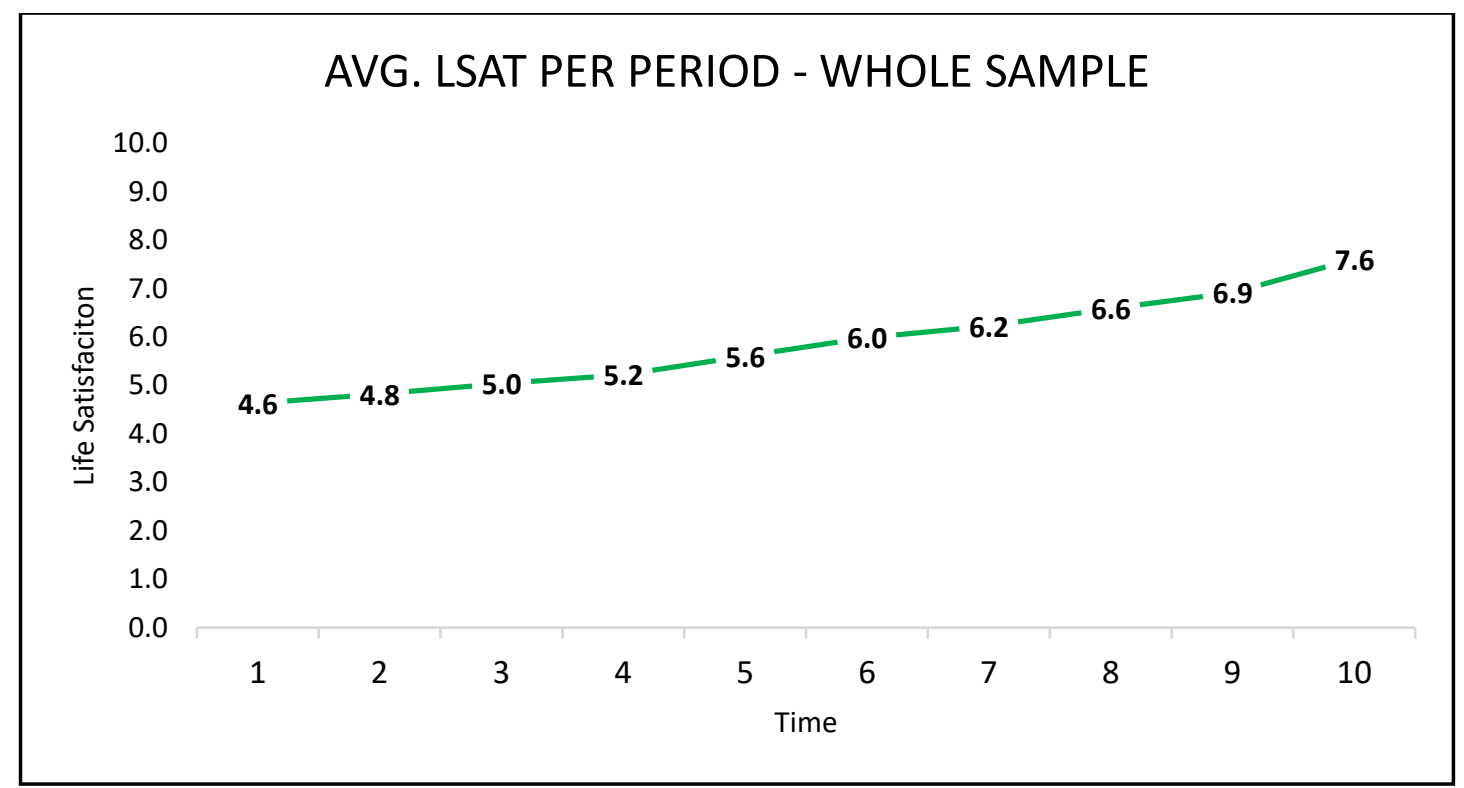

Figure 6: Average life satisfaction period in HILDA—restricted sample

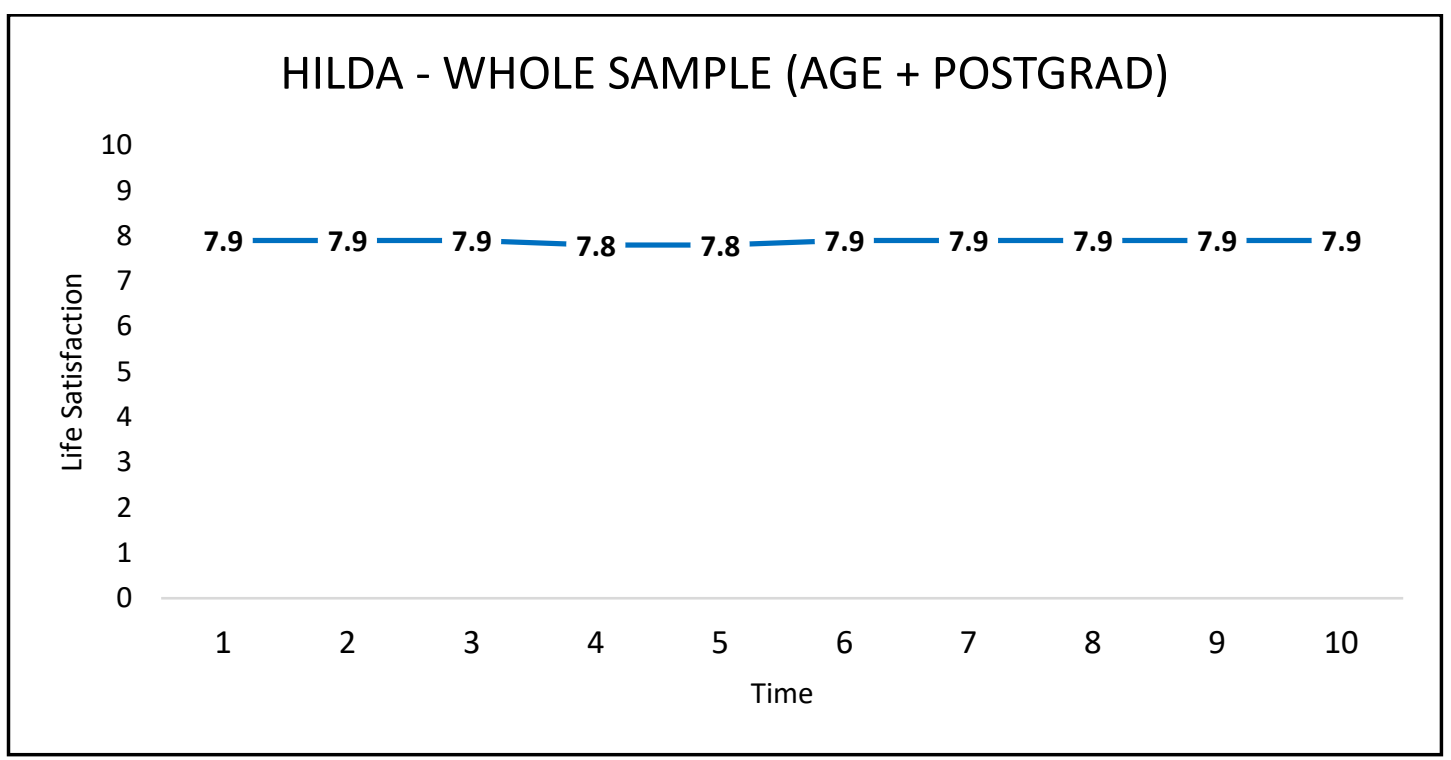

The lack of scale response change in HILDA despite evidence from the plotting study that life satisfaction is improving suggests that maybe scale norming is occurring, perhaps driven by ceiling effects given the high initial response of 7.9 in wave 1 . It is possible to explore potential scale norming in the plotting study by comparing plots to scale responses.

Specifically, a respondent with strong movement in one direction in their plot who does not show any change in their scale response is likely to be scale norming over time. To explore this in the plot data I begin with a loose definition of scale-norming designed to catch any potential candidates and then gradually tighten the definition to weed out more reasonable 
responses. A benefit of this approach is that it is relatively honest. It makes it clear how tight the definition needs to be before the phenomenon disappears.

Is scale-norming something we should be concerned about?

Scale norming is initially defined as having an absolute value of netchange of greater than or equal to 2.5 but an absolute value of scale change of less than or equal to 1 . Note that the standard deviation of netchange is 3. As such, what this definition does is ask whether someone has had meaningful movement in their life satisfaction as plotted but this movement has not shown up proportionately in their scale responses. A graphical example drawn from the sample is depicted below (figure 7). The individual has a discrepancy in their plot of +5 but their scale response doesn't change at all from ten years ago to today. This makes little sense unless the individual is scale norming over time.

Figure 7: Example of scale-norming from plotting study

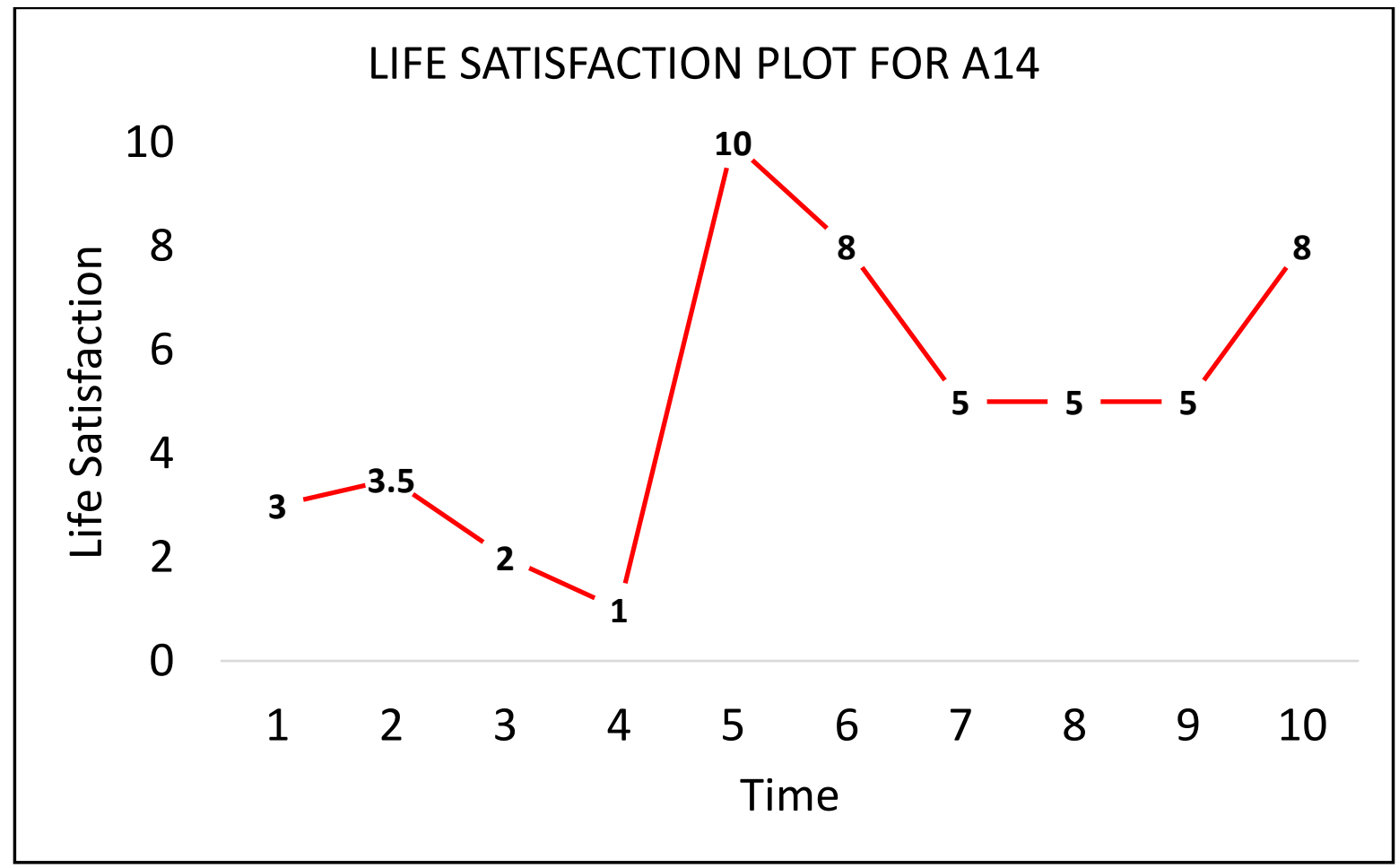

A14-Netchange: 5, Satisfaction "Then": 7, Satisfaction “Now": 7, Change: 0

The loose definition returns 36 candidates, but as expected many of these are making reasonable responses. The most common such reasonable response is a scale change of 1 for a netchange of 2.5 , which is in line with the regression results reported above. In the next step 
of the analysis, the definition is tightened to netchange of at least 3 (i.e. at least 1 standard deviation) and scale change of less than 1 (i.e. no movement). This leaves only 6 candidates. Some of these quite clearly show scale norming, such as one individual reporting a netchange of 9 and a scale change of 0 .

In a final step, a strict but wide definition of scale norming is used where respondents are considered to have scale-normed if they report more than 2 standard deviations of netchange (i.e. greater than 6) but no more than 1 unit of scale response change. Such individuals show a large change in their life satisfaction in their plots but not a proportionate change in their scale response. An example of such a respondent is described below (figure 8). This individual has a netchange of +9 but a scale change of only +1 . It is debatable whether these individuals are scale norming. In any case, this wider definition returns only an additional 4 candidates for a total of 10 scale-normers, or 7 per cent of the sample.

Figure 8: Example of scale-norming by wide definition

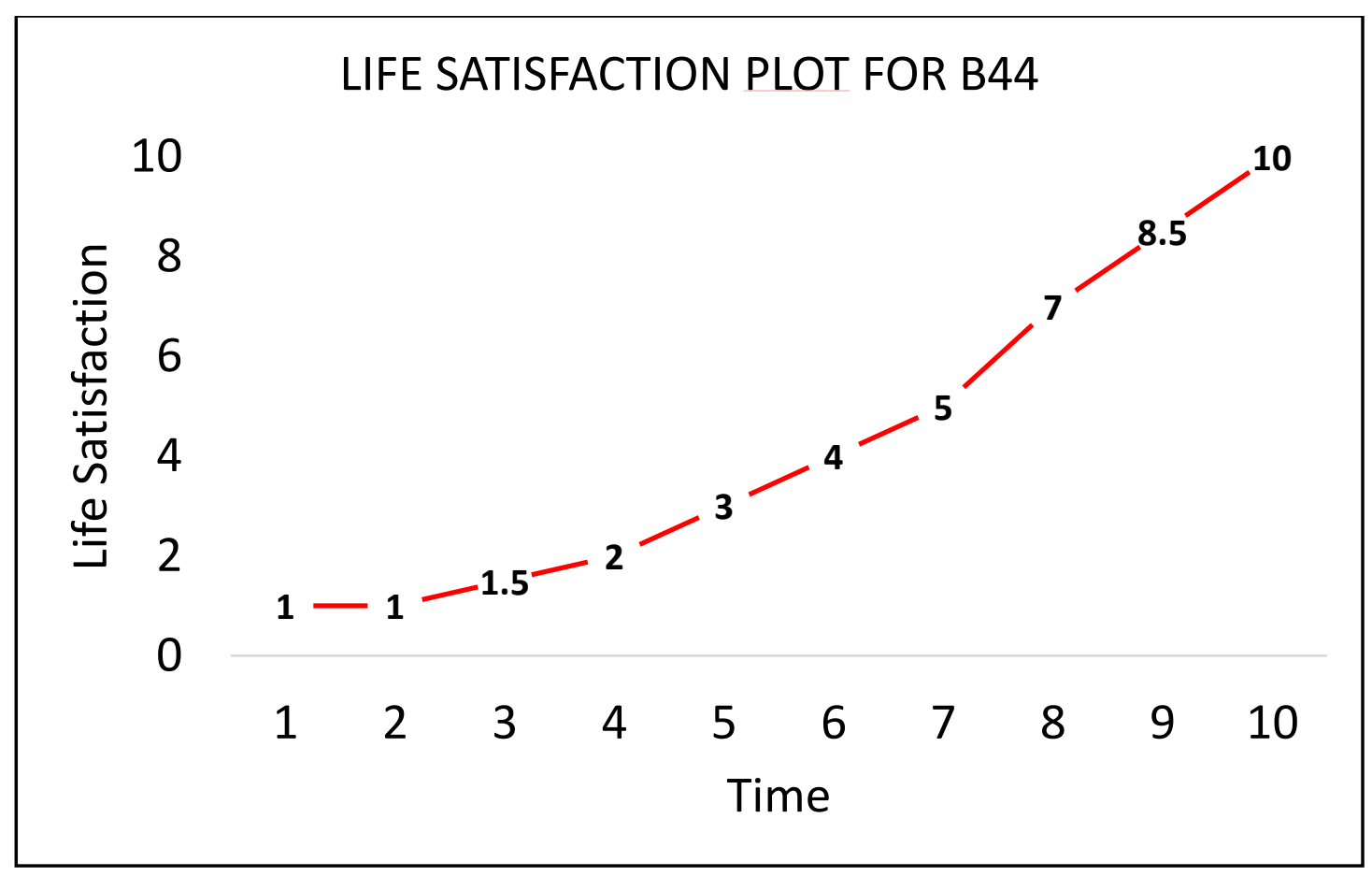

B44-Netchange: 9, Satisfaction “Then": 7, Satisfaction “Now”: 8, Change: 1

7 per cent doesn't seem too problematic, but there are additional candidates for scale-norming who have not yet been included-reversers. A reverser is someone whose scale response moves in the opposite direction to their plot. For example, the respondent depicted below (figure 9) showed an increase of life satisfaction in their plot of 4.5, but their scale response declined by -4 . This makes little sense unless the individual is scale-norming. It is unlikely 
that the respondents are simply misunderstanding the question as they are masters students at a global top 50 university.

Figure 9: Example of a reverser

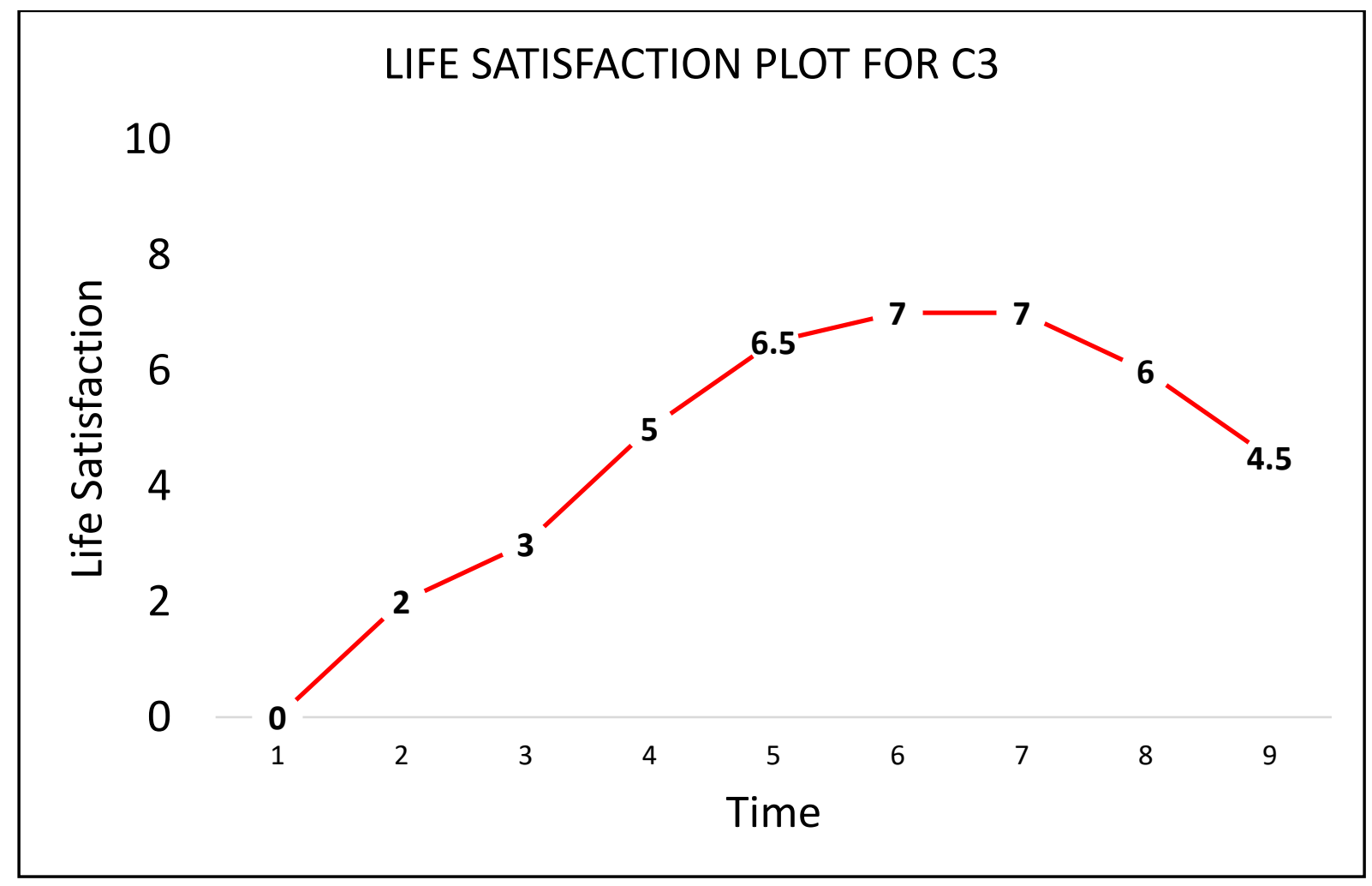

C3-Netchange: 4.5, Satisfaction “Then”: 8, Satisfaction “Now”: 4, Change: -4

There are 12 reversers in the sample. Adding them to the scale-norming group gives 22 people, or $15 \%$ of the sample. This $15 \%$ appear to be responding in inconsistent ways that support scale-norming. There is no way to identify them from their scale responses alone. This makes eliminating them and their contaminating effect from samples in life satisfaction studies using scales difficult. Their large number makes drawing inferences from the scale responses of such samples over time problematic.

It should be noted that this $15 \%$ is likely a conservative estimate. On the one hand, this is a purposeful sample where we would expect to find scale norming. On the other hand, people had the possibility in this study of making their "Life Satisfaction Then" response consistent with their plot, which might prime them to describe their life satisfaction 10 years ago as being lower than they would have said if asked at the time. In other words, given the nature of the instrument used here, it is possible that some respondents use the same scale to describe their life satisfaction today and ten years ago, whereas in a year on year study they 
would scale-norm. It would be helpful to include plot questions in existing longitudinal social surveys like HILDA and investigate the extent to which the shape of the plots corresponds to the last 10 years of scale responses of the survey participants. This would provide a clearer indication of scale norming because the scale responses would be from the actual point in time the plot is tracking.

\section{Is adaptation as strong as scale responses would have us believe?}

As with scale-norming, the investigation of adaptation in the plotting study data begins with the specification of a wide definition that should capture all relevant respondents. This definition is then gradually tightened. The initial loose definition for adaptation is at least one standard deviation of total change (4.5), but less than or equal to one standard deviation of netchange (i.e. 3) and less than or equal to 1 unit of absolute change in scale response. This should capture respondents who have experienced shocks to their life satisfaction over the course of the survey (the total change) but then adapted back to their set point over time (hence limited net change and scale change). An example respondent is depicted below (figure 10). They experience a substantially deterioration in their life satisfaction over the 10 years of the plot, but almost entirely adapts back to their original level by the end of the period. Incidentally, such quadratic shapes are somewhat common in the plotting study at $6 \%$ of the sample. This lends some support to the adaptation hypothesis, but the adaptation here is over a much longer time period than what we see in year-on-year scale studies, and the "shock" itself is also gradual.

The loose definition of adaptation picks up a large number of consistent responses, such as people with a netchange of 2.5 and a scale change of 1 . However, even with this very wide definition, only 15 candidates emerge, or $10 \%$ of the sample. 
Figure 10: Example of adaptation

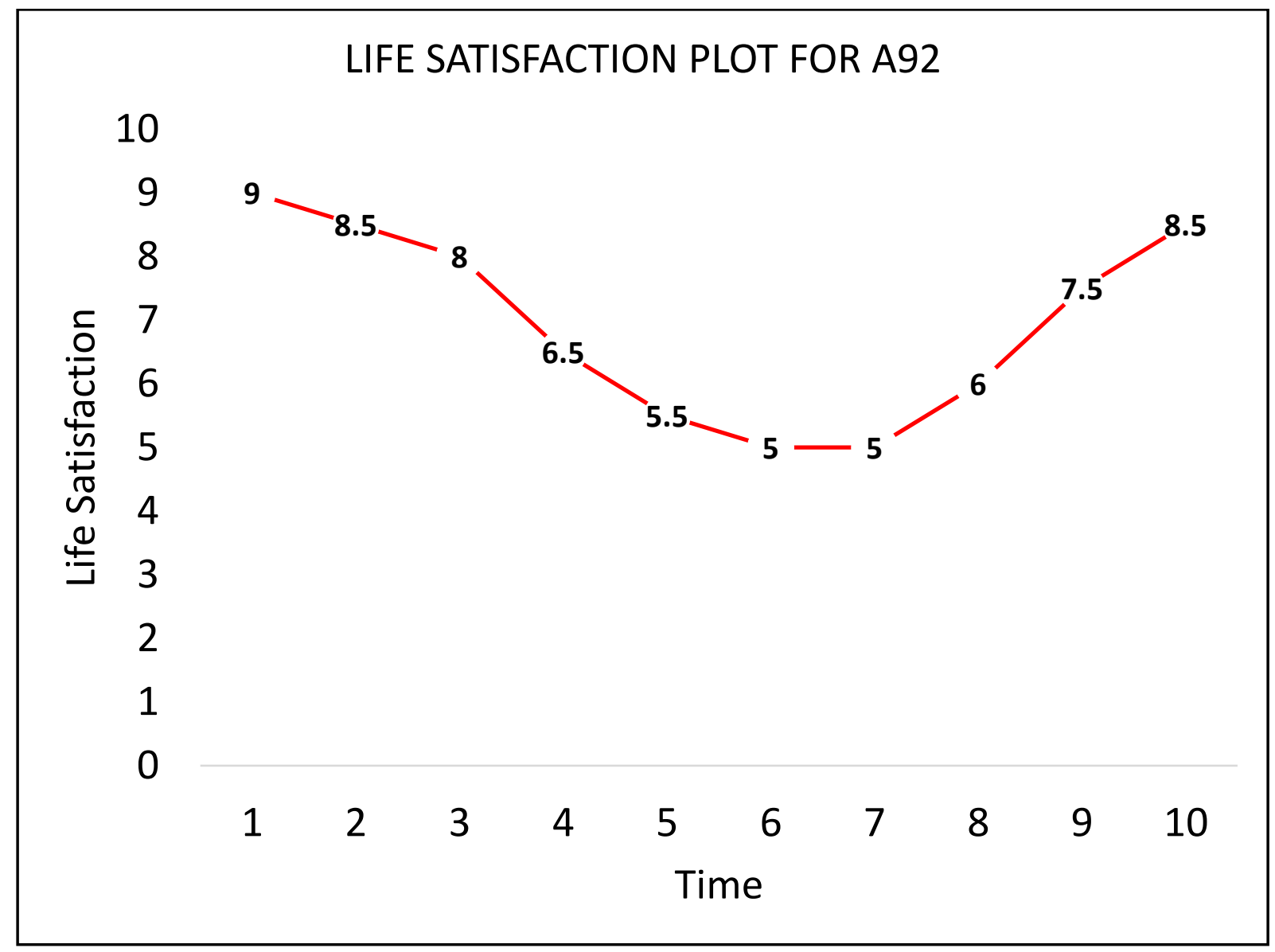

A92-Total Change: 7.5, Netchange: -0.5, Satisfaction “Then": 8.5, Satisfaction "Now": 8, Change: -0.5

The definition of adaptation is then tightened further by restricting the scale responses change to less than 1 . In this case, there are only 6 candidates, or $4 \%$ of the sample. If netchange is also restricted to 1 or less only 3 candidates remain. An example of this "strong adaptation" definition is presented below in figure 11. 
Figure 11: Example of "strong adaptation"

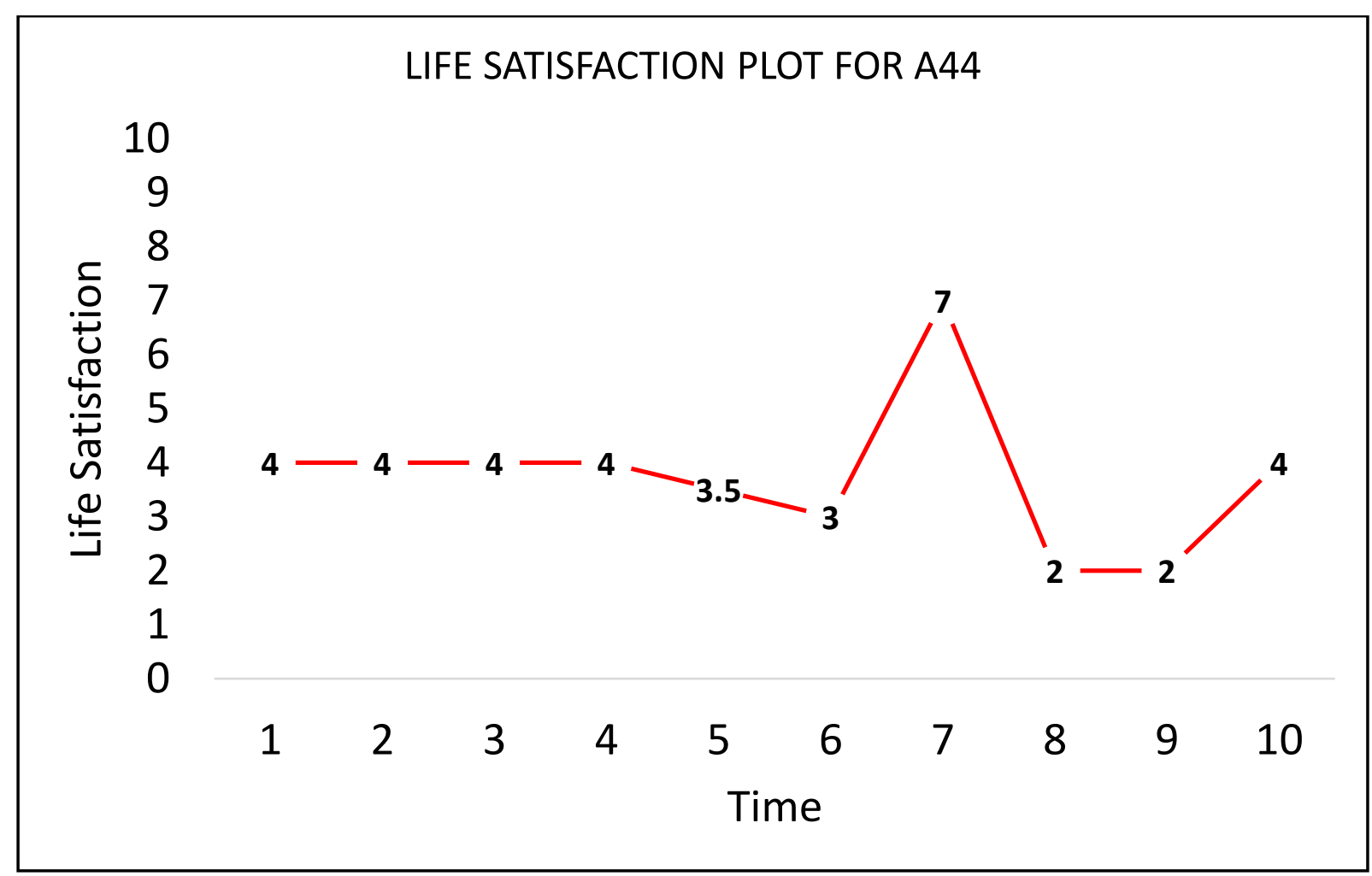

A44-Total Change: 12, Netchange: 0, Satisfaction “Then": 9, Satisfaction "Now": 9,

\section{Change: 0}

This definition might miss out individuals who experienced a large change and haven't had time to adapt yet. However, if the net is widened to include people with at least 2 standard deviations (9 units) of total change, less than 2 standard deviations (6) of netchange and less than 2 points of scale change, no new candidates emerge. The plotting study data does not seem to support the strong adaptation hypothesis.

\section{Discussion}

A common response to the results presented above is that they are simply a product of recall bias (Coughlin 1990, Kahneman 1999, Stone et al 1999). The plotting metric asks respondents to consider the present relative to the past, which raises concerns about errors of memory (Lucas 2016). However, recall bias seems a difficult charge to level in this case.

Stillman et al's study is a natural experiment. Why do only the migrants appear to suffer from recall bias? In the plotting study, individuals who appear to be scale norming are differentially recalling their scale response and their plot. If there was recall bias, we would 
expect it to at least be consistent across these two questions. The retrospective element of the plotting instrument is also relatively straightforward because it only requires the description of ordinal trends.

It is perhaps worth noting that responses to even simpler retrospective satisfaction questionnaires do not align with responses to year-on-year scale responses. The first four waves of the German Socio-Economic Panel (GSOEP) included scale question for present satisfaction and for satisfaction in the previous year. The correlation between the retrospective question and the actual response in the previous year is only 0.5. Similarly, waves 16 and 17 of the British Household Panel Survey (BHPS) include both a life satisfaction scale question and the following: "would you say you are more satisfied with life, less satisfied with life, or feel about the same as you did a year ago?". The correlation between these two variables is only 0.22 . The BHPS question is essentially a binary choice of the form "is your life better today than it was last year?" If we are worried about the cognitive complexity of such questions we should certainly be concerned about the cognitive complexity of responding to scale questions year-on-year in a way that effectively communicates dynamic change in satisfaction levels. This is especially the case given that life satisfaction scale questions themselves require some degree of recall. If they did not, then what they are measuring would not be conceptually different from what is measured in experience sampling methods.

Issues of recall bias are used to deflect concerns about scale-norming and defend the counterintuitive results of SWB research. Year-on-year scale responses are said to undermine retrospective evaluations, but one could just as easily say that retrospective evaluations undermine year-on-year scale responses. And while recall bias might explain the discrepancy between year-on-year scale questions and retrospective evaluations, scale norming is an equally valid explanation. Scale-norming has the added advantage of producing inferences that are in line with old intuitions, like the long-run positive impacts of economic growth. There is no particularly salient reason to prefer either view at this stage given that both sets of metrics involve difficult cognitive exercises. More research is required.

A second common response to the results herein is that it is incorrect to think of life satisfaction as a continuous variable. It is in fact bounded, just like pleasure, which goes from 0 (so painful you pass out) to 10 (so ecstatic that you pass out). This argument in no way undermines the results, as shown in figure 11, below. If life satisfaction is organismically 
bounded in the sense that humans just can't have more than $\chi$ satisfaction but satisfaction is still cardinal if only we could measure it, then the logic of the argument and interpretation of the results remains the same. This is demonstrated graphically below (figure 12). Even with an organismic bound (i.e. 10) that the individual can conceive from wave 1 of the survey, it remains the case that the individual may qualitatively alter their scale across waves in way that makes responses incomparable even ordinally.

\section{Figure 12: Organismically bounded life satisfaction}

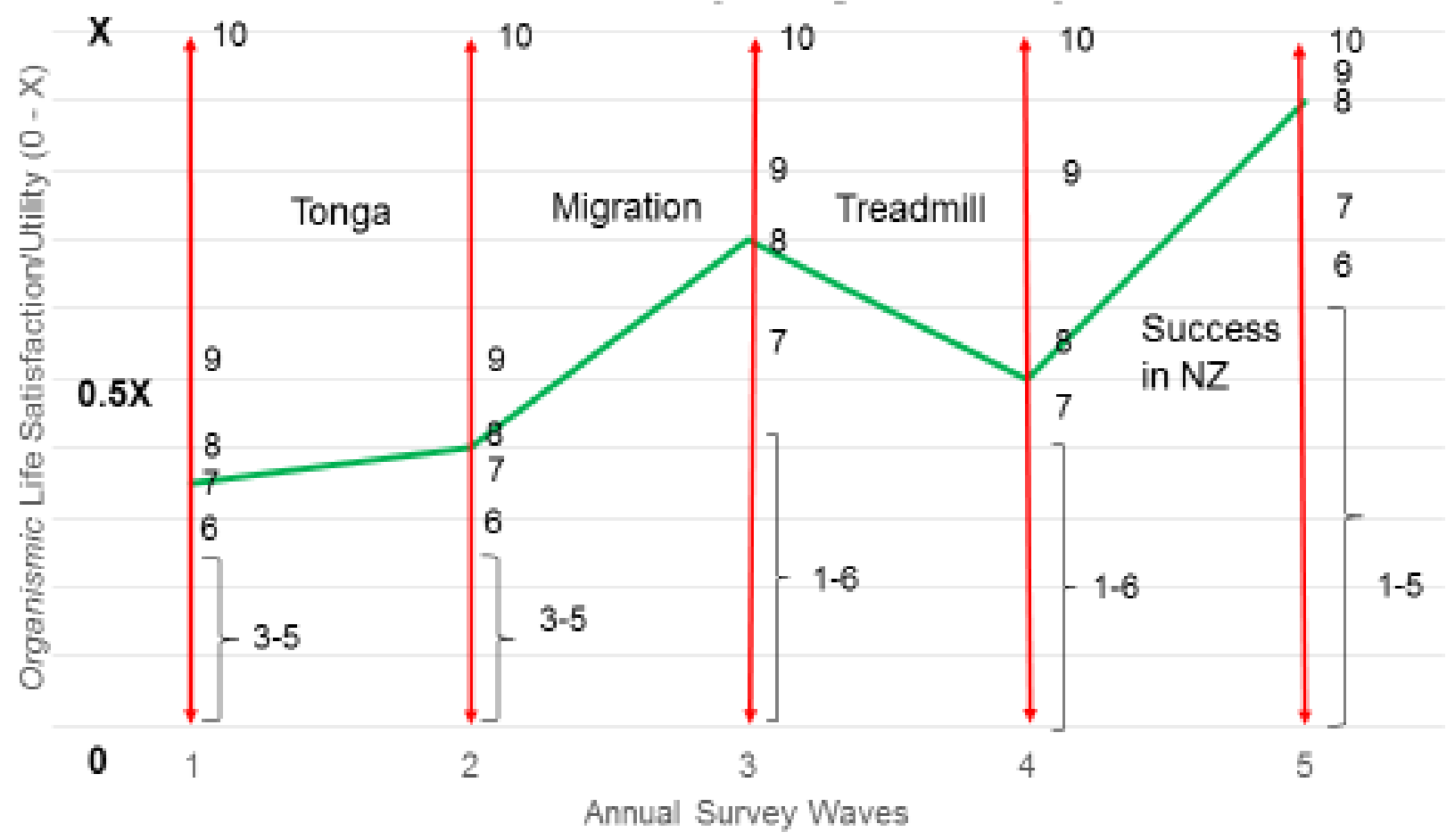

Incidentally, figure 12 also describes why scale-norming can affect responses to Cantril's ladder of life questions. Recall that these ask respondents to conceptualise their scale as bounded by their "worst possible life" and "best possible life". Even with a fixed conception of a 10/10 life that is way off in the distance, it is still possible for an individual to scale norm as they move towards this goal. For example, say that a particular Tongan migrant's best possible life is to be a minister in the New Zealand cabinet. Step 1 is to migrate, so achieving this results in an improvement in life satisfaction, but also a reassessment of the probability that this Tongan can make it to parliament, so they scale-norm upwards. Upon arriving in New Zealand, they also experience some adaptation owing to changing reference groupsthey are no longer elite. This reduces their absolute satisfaction, but not their scale response. A decade or two later, they are elected to parliament. This major move towards the 
individual's best possible life provokes a major change in life satisfaction. However, they can now also see explicit steps to becoming a minister, namely chairing some committees, so this now becomes their 9/10, whereas previously $9 / 10$ was simply getting elected. Certainly this person's aspirations have grown, but so has their life satisfaction. The two should not be confused. Adaptation due to rising aspirations and changing reference groups can occur simultaneously with scale-norming.

If life satisfaction is not organismically bounded but rather cognitively bounded then it becomes an open question whether life satisfaction scale responses can be used for welfare analysis. This conceptualisation of scale responses dispenses with cardinality entirely and instead conceives of scale responses as strictly ordinal. Consequently, it is correct to say that a frustrated millionaire in New York and a happy peasant in Kolkata who both say they are an $8 / 10$ are in fact equally satisfied with life, even if the peasant would like to trade places with the millionaire but the millionaire would not like to trade places with the peasant. In this case, life satisfaction scales cannot be used to decide policy priorities over, among other things, the distribution of public spending, as advocated by some happiness economists (Fujiwara and Dolan 2016, Clark et al 2018), because doing so would result in perverse outcomes. If scales are cognitively bounded, then it is reasonable to say that extroverts are on average more satisfied than introverts (Diener and Lucas 1999), but it is less reasonable to say that this is something we should care about. If we made all the introverts extroverted this would hardly make the world a "better" place. The frustrated millionaire and sad introvert examples point to a deep epistemic issue for subjective well-being research, namely, if life satisfaction is cognitively bounded, then can we really say it has anything to do with wellbeing?

It is worth underlining that what is at issue here is only the inter-personal and inter-temporal comparability of scale responses. It is quite possible that at any one point in time an individual can navigate the scope, ranking and calibration problems to produce an ordinal ranking of possible lives and situate their present life within that ranking (as shown in the graphic above). However, it is also possible that respondents execute this cognitive exercise in substantially different ways at different points in time and thus end up answering scale questions using different scales over time, making these responses incomparable. Scale responses then become an interesting tool for studying the cognitive exercise itself, but not for investigating determinants and dynamics of absolute life satisfaction or wellbeing. 


\section{Implications}

Some caveats are in order. The results presented above must be checked with bigger and more representative samples. It would also be useful to test the plotting instrument with the scale labelled from 1-10. This might reveal the extent to which the plots simply show movement within scale levels. It may be the case that 9 points of netchange in the plotted response, for example, is simply not enough to move some respondent's scales up a single unit.

Even with these caveats, some tentative conclusions are reasonable. The divergence between what we see in plotting data from what we see in longitudinal scale data suggests that we need to experiment with alternate metrics for assessing evaluated wellbeing, especially in the context of dynamic change. Life satisfaction scales have passed through extensive psychometric validation but they have not been as extensively validated relative to other possible options. It would be worthwhile at this juncture to experiment with other life satisfaction metrics given that scales produce counterintuitive results. In the meantime, we should be cautious about assuming that phenomena we observe in life satisfaction scale data are not artefactual in nature. We should be especially cautious about accepting strong adaptation as a defining characteristic of dynamic trends in evaluated wellbeing and be mindful of the potential for scale norming.

Given the potentially pernicious effects of scale norming on the usefulness of life satisfaction scales, the phenomenon demands wider and deeper investigation. This is not a call to abandon the use of scales, or even to exercise more caution in their use - most researchers are very cautious already. Rather, it is a call to recognize that life satisfaction scales have long been the subject of methodological concerns (Frederick and Lowenstein 1999, McClimans et al 2012, Kaminitz 2018). Subjective well-being scholarship had to push past these concerns to establish itself as a viable field with interesting and valuable insights to offer (Diener et al 2009). It has done so amply. Now that the field has garnered substantial credibility, it has the latitude to revisit these methodological issues and sort them out.

In the next, final section of this dissertation, I discuss another metric that could be used alongside plots to explore life satisfaction from a different angle: worms. I also detail a 
methodology by which worms could be used to estimate wellbeing functions of the sort presented in chapter 4 .

\section{Life Satisfaction Worms}

Worms are used in televised debates between Australian Prime Ministerial candidates. Here they are used to track audience approval for things the candidates say on stage. Audience members are given clickers. Using these they can indicate when they like or dislike what a candidate is saying. Below is an image of former Australian Prime Minister Tony Abbott during the 2013 election campaign. The worm is displayed at the bottom of the screen and shows how the speaker's appeal is trending.

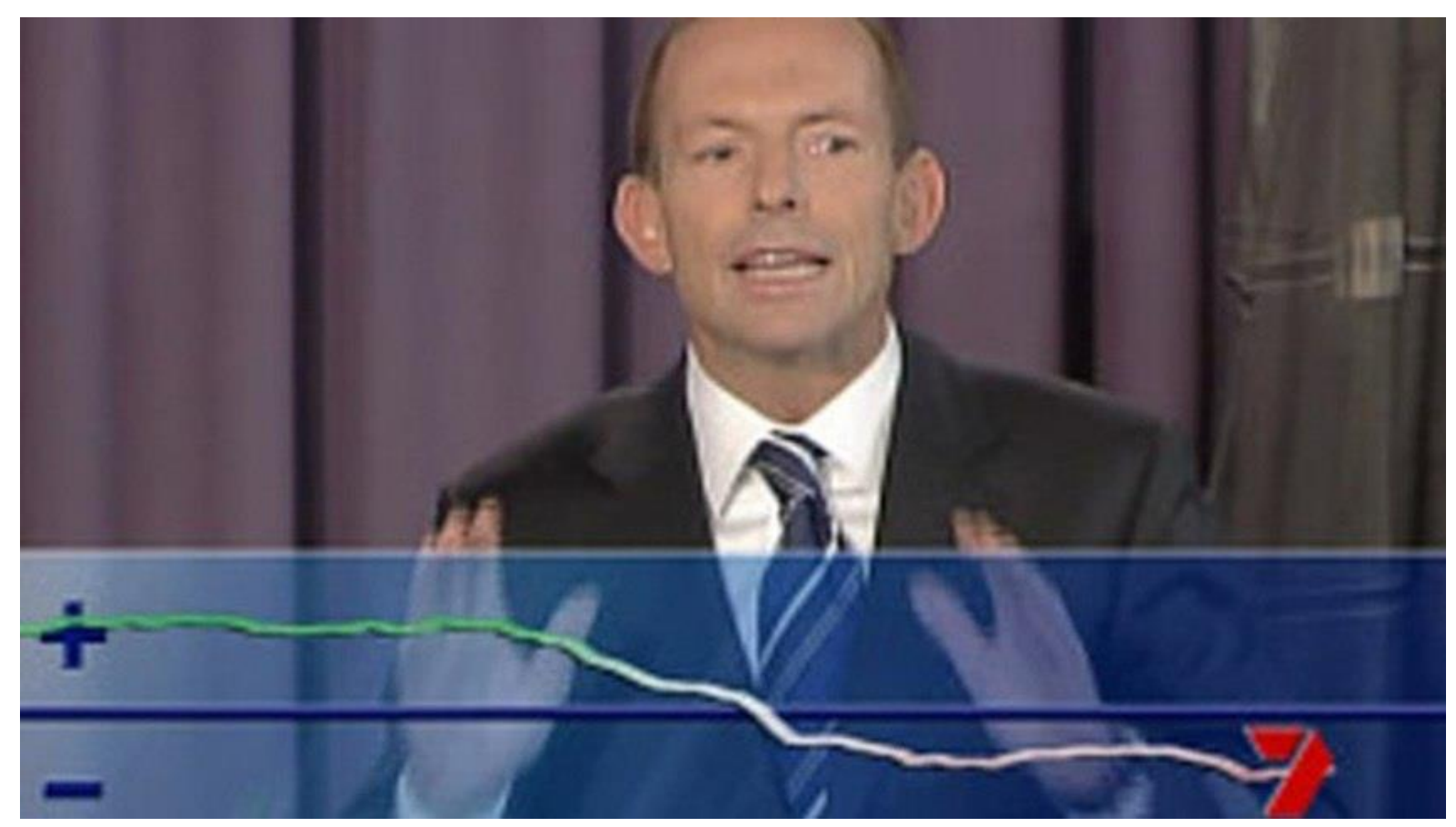

The worm measures the dynamics of appeal rather than absolute appeal. If someone had been saying appealing things for some time they would be well into the positive portion of the graph. If they start saying unappealing things at that point their worm will begin to trend downward, but these trends give little indication of overall appeal.

Worms could be useful in the context of SWB research because they can capture trends in life satisfaction in a quantifiable way without employing a strictly bounded scale. For example, respondents could be interviewed monthly and asked "would you say you are more, less or as satisfied with your life this month compared to last month?" If a respondent says "as 
satisfied" then their cumulative score for that month is +0 . If they are more or less satisfied they could get $\mathrm{a}+1$ or -1 if the researcher wanted to completely avoid scales, or be asked at that point for a scaled answer. For example, a follow-up question could be: "on a scale from 1-10, how much more or less satisfied are you this month compared to last month?”

Scale-norming would still be a concern when trying to compare the magnitudes of these changes, but some insights could be gleamed by including more qualitative questions. For example, a follow-up question could be: "In 160 characters or less (the length of a txt message), please describe what provoked this change in your satisfaction". With this question and a sufficiently large sample, candidates for major and minor effects on life satisfaction could be derived directly from individuals. This would be superior to the present method where researchers use shocks that exist coincidentally in longitudinal panels to study satisfaction dynamics. An example is the adaptation research on divorce, marriage and death. We may be missing important sources of satisfaction change and misunderstanding causation simply because we don't ask the relevant questions.

Worms would also allow for alternate approaches to the analysis of adaptation. If the strong adaptation hypothesis is true then we would expect to see similar trends in worms to those we see in plots of life satisfaction responses - a mostly flat worm with rapid returns to trend following any shocks. In contrast, if adaptation is weak or incomplete and scale-norming is instead a major driver of trends in scale responses then we might expect to see structural breaks in life satisfaction worms instead. These would look something like this (figure 13):

Figure 13: Life satisfaction worm with structural breaks

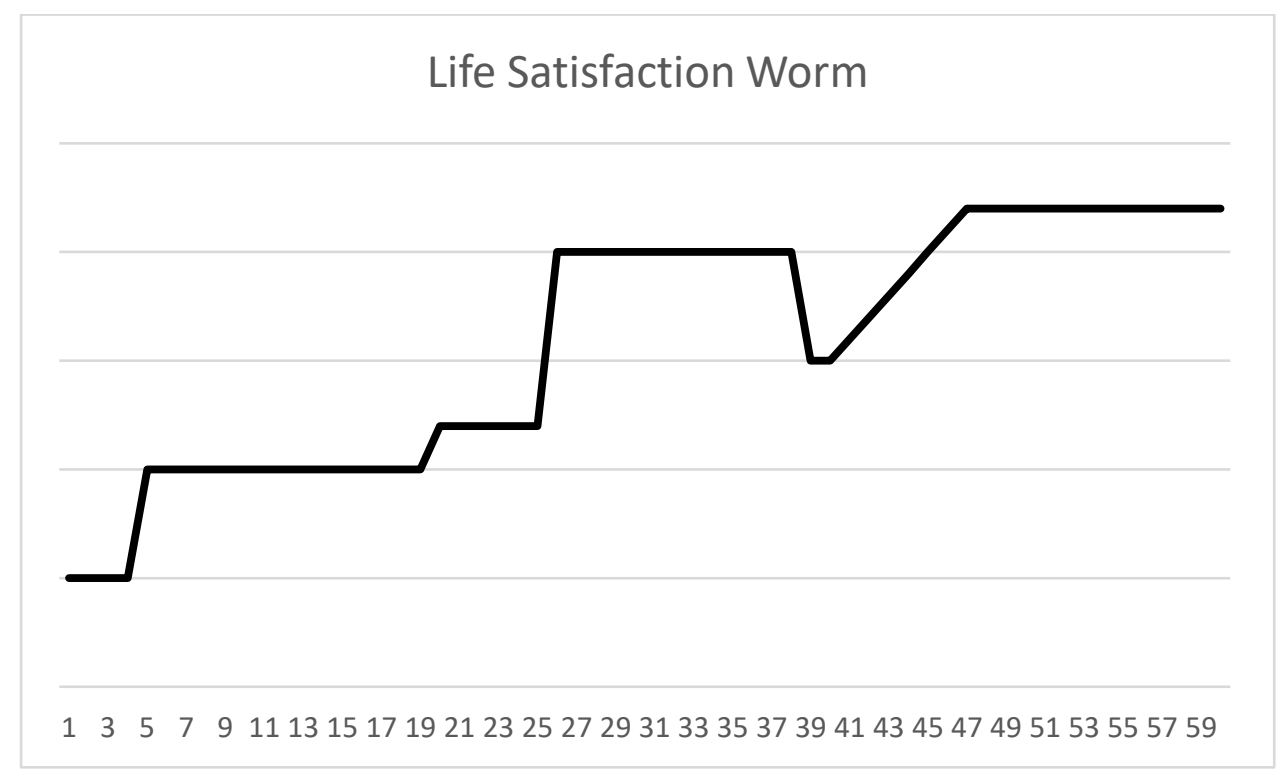


The period from $t_{39}$ to $t_{47}$ represents a period of adaptation to a negative shock. We would here expect a respondent to say they are more satisfied than last period each wave, but only give a small improvement each wave. Cognitive adaptation could be disentangled from actual changes in circumstances by using the qualitative response question and/or by embedding the worm and qualitative response question in a larger social survey that tracks objective changes in circumstances like income, divorce or migration. The other changes in life satisfaction are large shocks associated with, mostly likely, goal achievement. The break at $\mathrm{t}_{4}$, for example, might represent being accepted to college. The break at $\mathrm{t}_{19}$ might represent being shortlisted for a prestigious graduate scholarship (a small increase in satisfaction), while the larger break at $t_{26}$ might represent actually getting that scholarship. In between these breaks life basically goes on with little noteworthy to report, and so the individual's satisfaction stays the same.

Worms might overcome some problems associated with assessing dynamic change in life satisfaction, notably scale-norming. However, they, like all other metrics of subjective wellbeing in contemporary usage, are insufficient to capture a critical domain of information, namely values and how they change over time. Without information on values - things like goals or preferences over income versus meaning - it is difficult to understand where wellbeing is coming from and its causal structure. To illustrate, recall that affective signals guide the process by which eudaimonia and despair are satisfied, and that aspects of the eudaimonia and despair clusters are often necessary for understanding why positive and negative mood persists. As such, if we only have a global measure of wellbeing rather than something that can be broken down into these component parts we will struggle to understand why wellbeing changes.

There are techniques in existence that overcome this issue to an extent. For example, anthropologists working with minority communities have developed qualitative research methods from which they, in cooperation with the community, can derive surveys that assess satisfaction with regards to things that the community specifically cares about (see Durie 2006, Ganesharajah 2009, McGregor et al 2003, Ruttenberg 2013, Yap and Yu 2016 for examples). These methods need to be restarted at regular intervals to allow up-to-date community values to be reflected in survey questions. While these methods show a great deal of promise, they are also administratively laborious, expensive and time consuming. This makes them ill-suited to many applications in public policy. 
One solution to this is to give respondents a smaller, fixed list of things to care about and then give them a number of points to distribute across this list to communicate their relative preference for each item. These lists can be derived from general theory or simple social surveys. I will illustrate this method using items drawn from the wellbeing production function. This example will thereby show how the wellbeing production function might be measured empirically and estimated statistically (if inaccurately).

At the beginning of the worm survey outlined above, respondents could be presented with the following 11 domains: freedom, money, health, education, environment, mood, friendships/family/community, competence, identity and self-knowledge, meaning and purpose, and morality. These domains correspond to the different variables of the well-being production function and the capabilities constraint. They could be described to respondents as follows:

Freedom: this is primarily about how autonomous you feel in your life. It captures whether you are politically enfranchised, free from coercion by your peers, family and the institutions you interact with, self-determined in how you spend your time, and able to pursue the things you value.

Money: How wealthy are you relatively to how wealthy you would like to be? Do you have enough money to do the things you would like to do?

Health: both mental and physical, including how fit you are. Are you in pain or debilitated? Are you physically and mentally able to do the things you would like to do?

Education: this includes both formal training and more general knowledge of the world and things that you might like to do. Do you have the qualifications you need to pursue your interests? Do you know the things you would like to know?

Environment: This refers to the liveability of your world, especially your immediate environment. In includes things like green spaces and opportunities to get into nature as well as aspects of the built environment that you value, like things to do and places to see. It also includes things like noise pollution and bad neighbours. 
Mood: simplistically, this is about whether you have a balance of positive over negative affect. Are you optimistic, chuffed, upbeat and generally 'happy' more often than you are anxious, depressed, irritable, angry or sad?

Friendships, family and community: do you feel like you have a good quantity and quality of relationships with people that you care about?

Competence: Are you good at the things you want to be good at? Do you feel like you are good at your life?

Identity and self-knowledge: this domain captures the extent to which you are self-aware and know who you are. Do you have a clear sense of self including things like your values and why you hold them?

Meaning and purpose: do you feel like your life is meaningful and serves a purpose that motivates you?

Morality: Are you a good person according to yourself and others?

There are 11 domains. Survey participants could be given 44 points to distribute across these domains on scales from 1-7. More points into a domain reflects a greater degree of importance placed on that domain. Respondents can either give everything a 4/7 corresponding to "neither relatively important nor unimportant" or down-weight some domains as relatively unimportant to up-weight others domains as relatively important. A sample answer might be the following (figure 14): 
Figure 14: Relative preference over subjective well-being domains

\begin{tabular}{|l|l|}
\hline \multicolumn{1}{|c|}{ DOMAIN } & \multicolumn{1}{|c|}{ PREFERENCE } \\
\hline Freedom & $* * * * *$ \\
\hline Money & $*$ \\
\hline Health & $* * * *$ \\
\hline Education & $* * * *$ \\
\hline Environment & $* * *$ \\
\hline Mood & $* *$ \\
\hline Friendships, Family and Community & $* * *$ \\
\hline Competence & $* * * * *$ \\
\hline Identity and self-knowledge & $* * * * *$ \\
\hline Meaning and purpose & $* * * * *$ \\
\hline Morality & $* * * * *$ \\
\hline
\end{tabular}

Respondents could then be asked to rate their satisfaction with these domains on a scale from 1-7. A sample answer at this stage might be the following (figure 15): 
Figure 15: Satisfaction with SWB domains

\begin{tabular}{|l|l|}
\hline \multicolumn{1}{|c|}{ DOMAIN } & \\
\hline Freedom & 7 \\
\hline Money & 5 \\
\hline Health & 5 \\
\hline Education & 6 \\
\hline Environment & 5 \\
\hline Mood & 5 \\
\hline Friendships, Family and Community & 4 \\
\hline Competence & 6 \\
\hline Identity and self-knowledge & 6 \\
\hline Meaning and purpose & 4 \\
\hline Morality & 6 \\
\hline
\end{tabular}

Respondents could then be asked the worm question at each survey wave and also asked whether either their domain preferences or domain satisfactions have changed. Combined with qualitative responses, researchers could assess which of the domains is driving changes in subjective wellbeing. At the conclusion of the study, it would be relatively straightforward (if somewhat fraught owing to scale-norming issues) to estimate wellbeing functions for all participants. It would be less straightforward but nonetheless possible to also analyse how individual's domain preferences changed over the course of the study and why. This would provide some preliminary insights into how people learn what values are right for them, though of course a deeper analysis of coalescence would require individual level data about specific goals.

The domain preferences and satisfactions methodology outlined above is only slightly more laborious to implement than than life satisfaction scales. While there are more questions initially, adjusting values in subsequent waves takes little time at all. The primary scale question is replaced by the worm question, which has 2 parts instead of just one. Hardly an imposition. The most important benefit of this method overall is that it would move the field away from grand averaging and allow for greater control over heterogeneity among 
respondents. For example, it would be possible to assess the impact of income on the life satisfaction of people who care about income versus people who do not using the interaction between preference for wealth and satisfaction with wealth, especially if objective measures of wealth are also available as part of a social survey. It would also be possible to assess whether there is a satiation point for income growth because the preference for income would decline at this point even as satisfaction remained high.

\section{Conclusion}

To effectively measure wellbeing in a policy context we need metrics that produce quantitative data that are also quick and thus cheap to implement. For these metrics to capture SWB in general they must also be a global measure rather than a measure of a specific dimension of wellbeing. To date, life satisfaction scales have been the primary metric employed to this end. Unfortunately, concerns around scale norming make these metrics problematic for analysing dynamic trends in life satisfaction over time.

One way to potentially overcome this weakness is to use life satisfaction plots or worms. However, these instruments, like life satisfaction scales, are somewhat clumsy if they are not augmented with additional questions that measure or account for things like goals, values and preferences. While tools for incorporating these items into SWB measures exist, they are typically laborious and expensive, making them difficult to employ in a large-scale policy context. Short qualitative questions are one solution, but these are limited in their interpersonal comparability.

One avenue to explore that could work in tandem with qualitative questions is to give respondents a list of possible sources of wellbeing change, including goals and values, and ask them to use these to indicate how and why their wellbeing is changing. This would give rise to quantitative, interpersonally comparable data with relatively limited concerns about scale-norming and some accounting for preferences, goals and values without sacrificing parsimony. The data would have limited use in welfare economics, but potentially wideranging applications in behavioural economics and policy more broadly. Subjective wellbeing research has not had the latitude to experiment with such potentially flawed metrics to date because it has been under tremendous pressure to establish itself as a credible field. Now that it is so established, and now that it is armed with a clear and comprehensive model of 
SWB thanks to this dissertation, it is time for the field to engage more deeply with these methodological issues. In this way SWB can move from being a field science to a theorydriven science. 


\section{Conclusion}

To think is easy. To act is hard. But the hardest thing of all is to act in accordance with your thinking.

- Johann Wolfgang von Goethe

This thesis was motivated by the challenges SW-B faces in making constructive and meaningful contributions to welfare economics and public policy. As argued in chapter 1, the paradigm of SW-B has made substantial contributions to science over the past four decades. It has illuminated the distinction between evaluated and experience wellbeing. It has raised new topics for inquiry, such as adaptation, set points, and the subjective wellbeing reporting function. And perhaps most importantly, it has contributed to the development of an architecture for the empirical study of SWB by peeling the concept away from discussions of prudential value. Hedonic psychologists and happiness economists defined a new constructSW-B - that could be studied without asking whether SW-B is "good-for" people. They utilised an exploratory empirical methodology and discovered a fascinating landscape for analysis. In all this, SW-B's operationalist epistemology was arguably a boon. But in the public policy and welfare economics domains, operationalism leaves SW-B scholarship open to longstanding and well-rehearsed critiques. This is in large part because wellbeing in these domains is intimately tied to prudential matters. Maximising wellbeing in policy and welfare economics means maximising prudential value, so to apply SW-B herein it must be asked whether SW-B is "good-for" people. The answer, for now at least, appears to be no. Prioritising SW-B could lead to perverse redistribution towards millionaires, clumsy allocation of public funds in cost-benefit analysis owing to scale-norming and other errors of measurement, and the blinkered prioritisation of hedonic dimensions of SWB at the expense of other important dimensions like eudaimonia. 
Nonetheless, SW-B has valuable contributions to make to these discourses, particularly with regards to empiricism. To make these contributions constructively and effectively, SW-B must replace its operationalist epistemology with a realist one. Chapter 2 argued that the first step in this enterprise is to posit a deep and comprehensive theory of SWB, including how it relates to the prudential good. I have provided such a theory in this dissertation, merging the SW-B literature with the broader literatures on SWB and wellbeing in psychology, philosophy and economics. This theory can now be used to inform the development of new metrics for empirically analysing SWB. As the wellbeing production function and coalescence of being theory of SWB is "fully articulated and defended against alternatives" it can be used to drive construct validation exercises as per Alexandrova's (2017, p. 150) specification of a "better implicit logic" thereof.

The theory outlined in chapters 4-9 posits that SWB must be understood in two parts: as an outcome and as a process. The state of SWB is modelled by the wellbeing production function, which defines SWB as a function of hedonia, eudaimonia and despair. These dimensions correspond roughly to whether one feels one's life to be pleasant, fulfilling and valuable, respectively. Drawing on the literature in SW-B and philosophy, chapter 6 argued that hedonia is a function of positive affect, negative affect, hedonic life satisfaction and emotional disposition. There are a range of techniques for improving this dimension of wellbeing, including savouring, gratitude and mindfulness. Chapter 7 distinguished eudaimonia from hedonia using theoretical arguments and empirical evidence from clinical and social psychology. It also defined eudaimonia as a state wherein an individual's basic psychological needs for autonomy, competence and relatedness are thoroughly nourished. Chapter 8 argued at length that the despair domain was a crucial element of wellbeing that needs to be better appreciated by SWB scholars. Despair concerns meaning and purpose, identity and a sense of normative seriousness. While empirical inquiry in psychology has advanced our factual understanding of despair in recent decades, existential philosophy, where the concept of despair was first developed, still has a lot of depth to offer and should be more thoroughly integrated into philosophical contributions to SWB scholarship.

The dimensions of SWB are interconnected. Affective signals from the hedonic dimension guide the coalescence of being that nourishes the basic psychological needs in the eudaimonia dimension and secures the meaning, seriousness and identity that comprise the despair dimension. In turn, it is only by improving eudaimonia and despair that the individual can achieve a life that is punctuated predominantly by positive affect rather than negative 
affect. Eudaimonia and despair are connected as well. The nihilist, who knows little of themselves and perceives the world as pointless and morality as flippant, will struggle to motivate themselves and nourish their basic psychological needs. Meanwhile, individuals raised in environments that foster their basic psychological needs, particularly for relatedness, will be buffered against the debilitating effects of despair.

Individuals seeking to improve their SWB are constrained in this activity by their capabilities. These capture the extent to which individuals can be who they want to be and do what they want to do. Chapter 5 explained the contents of the capabilities constraint drawing on the work of Sen (1999a, 1999b) and Nussbaum (2000) and the way their ideas have been operationalised in the HDI, MDGs and SDGs. Capabilities include income, health, education, political enfranchisement and environmental quality. A central claim of chapter 5 was that once an individual has sufficient capabilities to be who they want to be and do what they want to do, increasing their capabilities will have little impact on their wellbeing. If an individual appears to have a relaxed capabilities constraint but is nonetheless unwell, it is possibly because they lack quality information regarding who they should be and what they should do. Understanding this information shortfall in a comprehensive fashion necessitates having some model of how individuals come to learn what ways of being and doing are best for their unique selves. The coalescence of being, explored in chapter 9, provides insights into what this learning process involves.

The coalescence of being is a model of self-actualisation, a long running fixture of philosophical accounts of wellbeing that has until now been somewhat underspecified. The central process of coalescence is the harmonisation of the actual, ideal and ought selves. This correspond to who one is, who one wants to be, and who one has a responsibility to be, respectively. The ideal and ought selves consist of goals and identified values. Individuals trying to become their ideal selves will occasionally meet with success. They will then be disclosed as who they would like to be. This will be an exhilarating moment laden with positive affect. More generally, positive affective signals will punctuate progress towards goals that are self-concordant, indicating that the individual is on the right path towards SWB. Negative affective signals require introspection and rational digestion. They may indicate that the individual is not pursuing their goals with enough rigor and consequently falling short of sensibly desired ends. Or they may indicate that the ends are inappropriate. They may be self-discordant: a poor fit for the individual in question. The individual must then abandon these goals and reconceptualise their ideal and/or ought self. 
This thesis argued that as coalescence progress the individual will slowly improve their scores across all domains of SWB. They will pursue predominantly intrinsically motivated activities, nourishing their sense of autonomy. They will comport towards activities and values that are associated with positive affect and abandon those associated with negative affect, ensuring hedonia in the long run. Their progress towards their ideal self will give them a sense of growing competence, and their progress towards their ought self will give them a sense of meaning and purpose, especially as they change the world to be more in line with their values. Pursuit of the ideal and ought selves will almost inevitably bring the individual into communities of like-minded individuals where will be valued, nourishing their sense of relatedness. Introspection upon the affective signals that guide the individual along the path of coalescence will give the individual a rationally accessible understanding of themselves, providing identity and ameliorating anguish. Finally, coalescence requires integrity and fealty towards the principles inherent in the ideal and ought selves. This means that these principles come to exert a binding power over the individual's behaviour that is emerges out of that individuals own desires. This ensures seriousness and grounds ethics in self-interest.

The combination of the wellbeing production function and the coalescence of being is a hybrid theory of wellbeing. It emphasises objective criteria, such as a preponderance of positive over negative affect and nourished psychological needs, which determine whether someone is well. However, it also admits a tremendous amount of heterogeneity in how individuals go about attaining their objective indicators. Furthermore, the theory emphasises that it is individuals who arbitrate over their ideal and ought selves, ponder their affective signals and decide what values and goals are right for them. In this sense, the theory includes a substantial subjectivist component not dissimilar to preference-satisfaction accounts of wellbeing that include notions of laundered preferences. The coalescence of being models what laundering entails and the wellbeing production function models what the state of someone with satisfied preference is like. The central role given to affective signals in guiding the laundering process and helping individuals to nurture their basic needs means that the theory has a substantial hedonistic component as well. Finally, the strong emphasis on process as well as outcome in the model means that it includes a eudaimonic element as well. It even includes a link to Aristotelian accounts of eudaimonia in the role it grants for reason in introspection and virtue in integrity, seriousness and the ought self.

The two-part theory of wellbeing presented in this dissertation argues that the prudential good must be understood as both an outcome and a process. Some people have ill-being while 
others have well-being, and they will report as much. No individual scoring low in all of the dimensions of the SWB production function will say they are well. Similarly, no individual scoring high in all of the dimensions of SWB will say they are unwell. These are empirical claims that are meaningfully verified by the empirical literature reviewed in chapters 6 through 8. Similarly, there is a right way and a wrong way to go about getting SWB. Individuals who, for example, pursue extrinsic aspirations are unlikely to report high levels of SWB because their lifestyle is unlikely to nurture their basic psychological needs nor provide them with many positive affective signals. The inverse is true for people who wisely prosecute the coalescence of being. As such, efforts in welfare economics and public policy that aim at maximising the prudential good must appreciate that it is not just a state but also a mode or manner of being.

Scholarship in the SW-B field has shown us that measuring SWB and analysing it empirically is immensely challenging but not impossible. Going forward, we should be cautious about any metric that seems quick and easy. At the same time, it is precisely quick and easy metrics that are needed for applications in policy and economics because of practical constraints relating to the costs of administering social surveys. I discussed these issues in chapter 10 . In this context, it is understandable that life satisfaction scales generated excitement and were enthusiastically pressed into service. Their use has certainly yielded some important insights, but they are also limited in their applicability. In particular, scales are a questionable metric for assessing the dynamics of SWB, especially among the well-off, owing to the problem of scale-norming. New metrics may be required to advance the field. Chapter 10 provided two candidates: life satisfaction plots and worms. These must also be complemented with metrics that capture people's values and goals so that these can be accounted for when analysing SWB reports. In the closing passages of the thesis I outlined how these new metrics could be used to empirically estimate the coefficients of the wellbeing production function.

Given the evidence for scale norming reviewed in chapter 10 and the arguments of Adler (2013) and Fleurbaey and Blanchet (2013) reviewed periodically throughout the volume, it seems reasonable to argue that SW-B theory and metrics are not appropriate, at least at this stage, for applications in welfare economics and public policy that require precise interpersonal comparisons of wellbeing. Cost benefit analysis and questions of distributive justice are the archetypal examples of such applications. The capabilities approach is more mature in this regard, both theoretically and empirically, and represents a major advance over paradigms based purely in monetary units (Alkire 2015, Fleurbaey and Blanchet 2013, ch. 6). 
Efforts should be concentrated there instead. In the meantime, research into advancing SWB to the point where it might helpfully contribute to such applications should be encouraged.

Advocates who want a greater role for SWB in public policy could focus on the numerous applications of SWB in domains that might be grounded under the heading of "behavioural policy”. Graham has been a leader in such applications in the economic space. She has studied, among other things, the relationship between SW-B and decision making in Peruvian slums and declining or aspiring communities in the United States (Graham 2011, 2017). Positive psychologists are even more active in this space. One example is designing policies for schools, prisons, workplaces, welfare centres and other institutions so that they support basic psychological needs (Jang et al 2010, Deci et al 2017). Another is implementing programs in such institutions to help people identify their personal strengths, thereby nurturing their sense of competence and helping them to define their identities (Seligman et al 2009). More broadly, SWB scholarship has valuable insights to offer on how to make civic institutions more accessible through deliberative or other mechanisms so that people feel capable of autonomously affecting their worlds (Ryan and Deci 2017, ch. 23). These applications of SWB are not the traditional purview of economics or the "social planner" but they are nonetheless in the domain of public policy. Furthermore, they are fields where economics and the paradigm of the social planner are not very useful because scarcity is not a pervasive consideration (Fabian 2018). As such, the heterodox insights of happiness economics and SWB more generally can potentially bring about substantial improvements. These behavioural domains are thus an ideal beachhead from which to build a more sustained and wide-ranging contribution to economics and public policy on the part of SWB. They are a relatively new field of inquiry and deserve to be the subject of future work. 


\section{Works Cited}

Abbe, A.; Tkach, C. and Lyubomirsky, S. (2003). The Art of Living by Dispositionally Happy People. Journal of Happiness Studies, vol. 4, no. 4, pp. 385-404

Adams, R. (1999). Finite and Infinite Goods. Oxford, UK: Oxford University Press

Adler, M. (2013). Happiness Surveys and Public Policy: What's the Use? Duke Law Journal, vol. 62, no. 8, pp. 1509-1601.

Alexandrova, A. (2017). A Philosophy for the Science of Well-Being. Oxford, UK: Oxford University Press.

Algoe, S. (2012). Find, Remind and Bind: The Functions of Gratitude in Everyday Relationships. Social and Personality Psychology Compass, vol. 6, no. 6, pp. 455-469

Alicke, M.; Guenther, C. and Zell, E. (2012). Social Self-Analysis: Constructing and Maintaining Personal Identity. In M. Leary and J. Tangney (eds.), Handbook of Self and Identity: Second Edition, pg. 291-308. New York, NY: Guildford

Alkire, S. (2016). The Capability Approach and Well-Being Measurement for Public Policy. In M. Adler and M. Fleurbaey (eds.), The Oxford Handbook of Well-Being and Public Policy, pp. 615-644. New York, NY: Oxford University Press

Aknin, L.; Dunn, E. and Norton, M. (2012). Happiness runs in a Circular Motion: Evidence for a Positive Feedback Loop between Prosocial Spending and Happiness. Journal of Happiness Studies, vol. 13, no. 2, pp. 347-355

Angner, E. (2015). Well-Being and Economics. In G. Fletcher (ed.), Routledge Handbook of Philosophy of WellBeing, pp. 492-503. London, UK: Routledge

Angyal, A. (1941). Foundations for a Science of Personality. New York, NY: Commonwealth Fund

Angus, L. and McLeod, J. (eds.) (2004). The Handbook of Narrative and Psychotherapy: Practice, Theory and Research. New York, NY: Sage

Annas, J. (1998). Virtue and Eudaimonism. Social Philosophy and Policy, vol. 15, no. 1, pp. 37-55

Annas, J. (2004). Happiness as Achievement. Daedulus, vol. 133, no. 2, pp. 44-51

Annas, J. (2011). Intelligent Virtue. New York, NY: Oxford University Press

Antonakis, J. (2017). On Doing Better Science: From the Thrill of Discovery to Policy Implications. The Leadership Quarterly, vol. 28, no. 1, pp. 5-21

Arampatzi, E., Burger, M. and Novik, N. (2018). Social Network Sites, Individual Social Capital, and Happiness. Journal of Happiness Studies, vol. 19, no. 1, pp. 99-122

Arendt, H. (1963/2006). Eichmann in Jerusalem: A Report on the Banality of Evil. New York, NY: Penguin

Argyle, M. (1999). Causes and Correlates of Happiness. In D. Kahneman, E. Diener and N. Schwartz (eds.), WellBeing: The Foundations of Hedonic Psychology, pp. 353-373. New York, NY: Russell Sage.

Argyle, M. and Lu, L. (1990). The Happiness of Extroverts. Personality and Individual Differences, vol. 11, no. 10, pp. 1011-1017

Argyle, M. (2001). The Psychology of Happiness, $2^{\text {nd }}$ Edition. London, UK: Routledge Aristotle (1999). Nichomachean Ethics. Trans. W. Ross. Kitchener, ON: Batoche Books

Armenta, C.; Jacobs Bao, K.; Lyubomirsky, S. and Sheldon, K. M. (2014). Is Lasting Change Possible? Lessons from the Hedonic Adaptation Prevention Model. In K. Sheldon and R. Lucas (eds.), Stability of Happiness: Theories and Evidence on Whether Happiness Can Change, pp. 57-74. London, UK: Academic Press.

Lucas (eds.), Stability of Wellbeing: Theory and Evidence on Whether Wellbeing Can Change, pp. 57-71. London, UK: Academic Press

Arrow, K., Dasgupta, P., Goulder, L., Mumford, K. and Oleson, K. (2012). Sustainability and the Measurement of Wealth. Environment and Development Economics, vol. 17, no. 3, pp. 317-353

Atkinson, A. (1970). On the Measurement of Inequality. Journal of Economic Theory, vol. 2, no. 3, pp. 244-263

Baard, P.; Deci, E. and Ryan, R. (2004). Intrinsic Need Satisfaction: A Motivational Basis of Performance and Well-Being in two Work Settings. Journal of Applied Social Psychology, vol. 34, no. 10, pp. 2045-2068

Bailey, T.; Eng, W.; Frisch. M. and Snyder, C. (2007). Hope and Optimism as Related to Life Satisfaction. The Journal of Positive Psychology, vol. 2, no. 3, pp. 168-175

Bakker, A. (2005). Flow Among Music Teachers and their Students: The Crossover of Peak Experiences. Journal of Vocational Behaviour, vol. 66, no. 1, pp. 26-44

Bargh, J. (2004). Being Here Now: Is Consciousness Necessary for Human Freedom? In J. Greenberg, S. Koole and T. Pyszczynski (eds.), Handbook of Experimental Existential Psychology, pp. 385-397. New York, NY:

Guildford. 
Bargh, J. and Chartrand, T. (1999). The Unbearable Automaticity of Being. American Psychologist, vol. 54, no. 7, pp. 462-479

Barnhofer, T.; Crane, C.; Hargus, E.; Amarasinghe, M.; Winder, R. and Williams, J. (2009). Mindfulness-Based Cognitive Therapy as a Treatment for Chronic Depression: A Preliminary Study. Behaviour Research and Theory, vol. 47, no. 5, pp. 366-373

Barkow, K.; Cosmides, L. and Tooby, J. (1992). The Adapted Mind: Evolutionary Psychology and the Generation of Culture. New York, NY: Oxford University Press

Bartlett, M. Y. and DeSteno, D. (2006). Gratitude and Prosocial Behaviour. Psychological Science, vol. 17, no. 4, pp. 319-325

Baumeister, R. (1992). Meanings of Life. New York, NY: Guilford Press.

Baumeister, R. (2005). The Cultural Animal: Human Nature, Meaning and Social Life. Oxford, UK: Oxford University Press

Baumeister, R. and Leary, M. (1995). The Need to Belong: Desire for Interpersonal Attachments as a Fundamental Human Motivation. Psychological Bulletin, vol. 117, no. 3, pp. 497-529

Beebe-Center, J. (1932). Psychology of Pleasantness and Unpleasantness. New York, NY: Van Nostrad

Beck, A.; Ward, C.; Mendelson, M.; Mock, J. and Erbaugh, J. (1961). An Inventory for Measuring Depression. Archives of General Psychiatry, vol. 4, no. 6, pp. 561-571

Belli, R.; Stafford, F. and Alwin, D. (2009). Calendar and Time Diary Methods in Life Course Research. Newbury Park, CA: Sage

Bentham, J. (1780/2007). An Introduction to the Principles of Morals and Legislation. Mineola, NY: Dover Publications Inc.

Besser-Jones, L. (2015). Eudaimonism. In G. Fletcher (ed.), The Routledge Handbook of Philosophy of Well-Being, pp. 187-196. London, UK: Routledge.

Bhuiyan, M. (2018). Life Satisfaction and Economic Position Relative to Neighbours: Perceptions versus Reality. Journal of Happiness Studies, vol. 19, no. 7, pp. 1935-1964

Boarini, R.; Comola, M.; Smith, C.; Manchin, R. and De Keulenaer, F. (2012). What Makes for a Better Life? The Determinants of Subjective Well-Being in OECD Countries, Evidence from the Gallup World Poll. OECD STD Working Paper.

Boehm, J. K.; Lyubomirsky, S.; and Sheldon, K. M. (2012) [The role of need satisfying emotions in a positively activity intervention], unpublished raw data, cited in: Shin L. and Lyubomirsky, S. (2014). Positive Activity Interventions for Mental Health Conditions: Basic Research and Clinical Applications. In J. Johnson and A. Wood (eds.), The Handbook of Positive Clinical Psychology, pp. 349-363. New York, NY: Wiley

Bond, T. and Lang, K. (forthcoming). The Sad Truth about Happiness Scales. Journal of Political Economy.

Bonebright, C.; Clay, D. and Ankenmann, R. (2000). The Relationship of Workaholism with Work-Life Conflict, Life Satisfaction and Purpose in Life. Journal of Counselling Psychology, vol. 47, no. 4, pp. 469-477

Bouchard, T. J.; Lykken, D. T.; McGue, M.; Segal, N. L. and Tellegen, A. (1990). Sources of Human Psychological Differences: The Minnesota Study of Twins Reared Apart. Science, vol. 12, pp. 223-28

Bourdieu, P. (1979). Distinction. London, UK: Routledge

Bower, G. (1981). Mood and Memory. American Psychologist, vol. 36, no. 2, pp. 129-148

Boyce, C.; Brown, G. and Moore, S. (2010). Money and Happiness: Rank of Income, Not Income, Affects Life Satisfaction. Psychological Science, vol. 21, no. 4, pp. 471-475

Boyce, C.; Wood, A. and Powdthavee, N. (2013). Is Personality Fixed? Personality Changes as Much as "Variable" Economic Factors and More Strongly Predicts Changes to Life Satisfaction. Social Indicators Research, vol. 111, no. 1, pp. 287-305

Boyce, C.; Wood, A. and Ferguson, E. (2016). Individual Differences in Loss Aversion: Conscientiousness Predicts How Life Satisfaction Responds to Losses Versus Gains in Income. Personality and Social Psychology Bulletin, vol. 42, no. 4, pp. 471-484

Boyce, C.; Wood, A.; Delaney, L. and Ferguson, E. (2017a). How do Personality and Social Structures Interact with Each Other to Predict Important Life Outcomes? The Importance of Accounting for Personality Change. European Journal of Personality, vol. 31, no. 3, pp. 279-290

Boyce, C.; Wood, A. and Ferguson, E. (2017b). For Better or Worse: The Moderating Effects of Personality on the Marriage Life Satisfaction Link. Personality and Individual Differences, vol. 97, no. 1, pp. 61-66

Boyce, C.; Delaney, L. and Wood, A. (2018). The Great Recession and Subjective Well-Being: How Did the Life Satisfaction of People Living in the UK Change Following the Financial Crisis? PLoS ONE, vol. 13, no. 8, pp. $1-17$

Bradburn, N. (1969). The Structure of Psychological Well-Being. Chicago, MI: Aldine 
Brickman, P.; Coates, D. and Janoff-Bulman, R. (1978). Lottery Winners and Accident Victims: Is Happiness Relative? Journal of Personality and Social Psychology, vol. 36, no. 8, pp. 917-927

Britton, P.; Van Orden, K.; Hirsch, J.; Niemiec, C. and Williams, G. (2014). Basic Psychological Needs, Suicidal Ideation, and Risk for Suicidal Behaviour in Young Adults. Suicide and Life Threatening Behaviour, vol. 44, no. 4 , pp. 362-371

Brown, G.; Gardner, J.; Oswald, A. and Qian, J. (2008). Does Wage Rank Affect Employee's Wellbeing? Industrial Relations, vol. 47, no. 3, pp. 355-389

Bruni, L. and Porta, P. (2005). Economics and Happiness: Framing the Analysis. Oxford, UK: Oxford University Press

Bruni, L. and Sugden, R. (2007). The Road Not Taken: How Psychology Was Removed from Economics, and How It Might Be Brought Back. The Economic Journal, vol. 117, no. 516, pp. 146-173

Bryant, F. and Veroff, J. (2006). Savouring: A New Model of Positive Experience. New York: Routledge (Psychology Press).

Burke, B.; Martens, A. and Faucher, E. (2010). Two Decades of Terror Management Theory: A Meta-Analysis of Mortality Salience Research. Personality and Social Psychology Review, vol. 14, no. 2, pp. 155-195

Burnham, T. (2003). Engineering Altruism: A Theoretical and Experimental Investigation of Anonymity and Gift Giving. Journal of Economic Behaviour and Organization, vol. 50, no. 1, pp. 133-144

Burnham, T. and Hare, B. (2007). Engineering Human Cooperation: Does Involuntary Neural Activation Increase Public Goods Contributions? Human Nature, vol. 18, no. 2, pp. 88-108

Campbell, J.; Trapnell, P.; Heine, S.; Katz, I.; Lavallee, L. and Lehman, D. (1996). Self-Concept Clarity: Measurement, Personality Correlates and Cultural Boundaries. Journal of Personality and Social Psychology, vol. 70, no. 6, pp. 141-156 Camus, A. (1942/2013). The Outsider. Trans. S. Smith. New York, NY: Penguin

Cardaciotto, L.; Herbert, J.; Forman, E.; Moitra, E. and Farrow, V. (2008). The Assessment of Present-Moment Awareness and Acceptance: The Philadelphia Mindfulness Scale. Assessment, vol. 15, no. 2, pp. 204-223

Carver, C. (2012). Self-Awareness. In M. Leary and J. Tangney (eds.), Handbook of Self and Identity: Second Edition, pp. 50-68. New York, NY: Guildford.

Carver, C.; Lawrence, J. and Scheier, M. (1999). Self-Discrepancies and Affect: Incorporating the Role of Feared Selves. Personality and Social Psychology Bulletin, vol. 25, no. 7, pp. 783-792

Chancellor, J. and Lyubomirsky, S. (2013). Humble Beginnings: Current Trends, State Perspectives and Hallmarks of Humility. Social and Personality Psychology Compass, vol. 7, no. 11, pp. 819-833

Chen, B.; Vansteenkiste, M.; Beyers, W.; Boone, L.; Deci, E.; Van der Kaap-Deeder, J.; Duriez, B.; Lens, W.; Matos, L.; Mouratidis, A.; Ryan, R.; Sheldon, K.; Shoenens, B.; Van Petegem, S. and Verstuyf, J. (2015). Basic Psychological Need Satisfaction, Need Frustration, and Need Strength across Four Cultures. Motivation and Emotion, vol. 39, no. 2, pp. 216-236

Church, A.; Katigbak, M.; Locke, K.; Zhang, H.; Shen, J.; de Jesus Vargas-Flores, J.; Ibáñez-Reyes, J.; TanakaMatsumi, J.; Curtis, G.; Cabrera, H.; Mastor, K.; Alvarez, J.; Ortiz, F.; Simon, Y. and Ching, C. (2013). Need Satisfaction and Well-Being: Testing Self-Determination Theory in Eight Cultures. Journal of Cross-Cultural Psychology, vol. 44, no. 4, pp. 507-534

Clark, A. (2006). A Note on Unhappiness and Unemployment Duration. Applied Economics Quarterly, vol. 52, no. 4, pp. 291-308

Clark, A. and Senik, C. (2011). Is Happiness Different from Flourishing? Cross-Country Evidence from the ESS. Revue d'Economie Politique, vol. 121, no. 1, pp. 17-34

Clark, A. and Senik, C. (2014a). Happiness and Economic Growth: Lessons from Developing Countries. Oxford, UK: Oxford University Press

Clark, A. and Senik, C. (2014b). Income Comparisons in Chinese Villages. In A. Clark and C. Senik, (2014). Happiness and economic growth, pp. 216 - 239. Oxford, UK: Oxford University Press

Clark, A.; Diener, E.; Goergellis, Y. and Lucas, R. (2008a). Lags and Leads in Life Satisfaction: A Test of the Baseline Hypothesis. Economic Journal, vol. 118, no. 529, pp. F222-F243

Clark, A.; Fritjers, P. and Shields, M. (2008b). Relative Income, Happiness and Utility: An Explanation for the Easterlin Paradox and Other Puzzles. Journal of Economic Literature, vol. 46, no. 1, pp. 95-144

Clark, A.; Flèche, S.; Layard, R.; Powdthavee, N. and Ward, G. (2018). The Origins of Happiness. Princeton, NJ: Princeton University Press

Cohen, L.; Pane, N. and Smith, H. (1997). Complexity of the Interpersonal Self and Affective Reactions to Interpersonal Stressors in Life and the Laboratory. Cognitive Therapy Research, vol. 21, no. 4, pp. 387-407 
Coleman, J. et al (1966). Equality of Education Opportunity. Washington, DC: National Centre for Educational Statistics.

Costanza, R., Alperovitz, G., Daly, H., Farley, J., Franco, C., Jackson, T., Kubiszewski, I., Schor, J. and Victor, P. (2012). Building a Sustainable and Desirable Economy-in-Society-in-Nature. New York, NY: United Nations Division for Sustainable Development.

Coughlin, S. (1990). Recall Bias in Epidemiologic Studies. Journal of Clinical Epidemiology, vol. 43, no. 1, pp. 87-91.

Crisp, R. (2006). Hedonism Reconsidered. Philosophy and Phenomenological Research, vol. 73, no. 3, pp. 619-645

Crocetti, E.; Rubini, M. and Meeus, W. (2008). Capturing the Dynamics of Identity Formation in Various Ethnic Groups: Development and Validation of a Three-Dimensional Model. Journal of Adolescence, vol. 31, no. 2, pp. 207-222.

Crocker, J. (2002). The Costs of Seeking Self-Esteem. Journal of Social Issues, vol. 58, no. 3, pp. 597-615

Crocker, J. and Park, L. (2004). The Costly Pursuit of Self-Esteem. Psychological Bulletin, vol. 130, no. 3, pp. 1275-1286

Crocker, J. and Park, L. (2012). Contingencies of Self-Worth. In M. Leary and J. Tangney (eds.), Handbook of Self and Identity: Second Edition, pp. 309-326. New York, NY: Guildford.

Cronbach, L. and Meehl, P. (1955). Construct Validity in Psychological Tests. Psychological Bulletin, vol. 52, no. 4, pp. 281-302

Crowley, K.; Schrager, J. and Siegler, R. (1997). Strategy Discovery as a Competitive Negotiation between Metacognitive and Associative Knowledge. Developmental Review, vol. 17, no. 4, pp. 462-489

Crowley, K. and Siegler, R. (1999). Explanation and Generalisation in Young Children's Strategy Learning. Child Development, vol. 70, no. 2, pp. 304-316

Csikszentmihaly (1992). Flow. New York, NY: Harper and Row

Cummins, R. (2014). Can Happiness Change? Theories and Evidence. In K. Sheldon and R. Lucas (eds.), Stability of Happiness: Theories and Evidence on Whether Happiness can Change, pp. 75-100. New York, NY: Academic Press.

Cummins, R.; Eckersley, J.; Pallant, J.; Vugt, J. and Misajon, R. (2003) Developing a National Index of Subjective Well-Being: The Australian Unity Wellbeing Index. Social Indicators Research, vol. 64, no. 2, pp. 159-190

Cummins, R.; Li, L.; Wooden, M. and Stokes, M. (2014). A Demonstration of Set-Points for Subjective WellBeing. Journal of Happiness Studies, vol. 15, no. 1, pp. 183-206

Daltroy, L; Larson, M.; Eaton, H.; Phillips, C. and Liang, M. (1999). Discrepancies between Self-Reported and Observed Physical Function in the Elderly: The Influence of Response Shift and Other Factors. Social Science and Medicine, vol. 48, no. 11, pp. 1549-1561

Daly, J. and Coates, B. (2018). Housing Affordability: Reimagining the Australian Dream. Melbourne, VIC: Grattan Institute

Damasio, A. (2010). Self Comes to Mind: Constructing the Conscious Brain. New York, NY: Vintage

Davies, W. (2015). The Happiness Industry: How the Government and Big Business Sold Us Well-Being. New York, NY: Verso.

Davis, D.; Worthington, E. and Hook, J. (2010). Humility: Review of Measurement Strategies and Conceptualization as Personality Judgement. The Journal of Positive Psychology, vol. 5, no. 4, pp. 243 - 252

Deaton, A. (2013). The Great Escape: Health, Wealth and the Origins of Inequality. Princeton, NJ: Princeton University Press

De Beauvoir, S. (1947/2002). The Ethics of Ambiguity. Trans. B. Frechtman. New York, NY: Kensington De Beauvoir, S. (1949/2011). The Second Sex. New York, NY: Vintage Books

De Brigard, F. (2010). If You Like It, Does It Matter If It's Real? Philosophical Psychology, vol. 23, no. 1, pp. 4357.

DeCharms, R. (1968). Personal Causation: The Internal Affective Determinants of Behaviour. New York, NY: Academic Press

Dechesne, M. and Kruglanski, A. (2004). Terror's Epistemic Consequences: Existential Threat and the Quest for Certainty and Closure. In J. Greenberg, S. Koole and T. Pyszczynski (eds.), Handbook of Experimental Existential Psychology, pp. 247-262. New York, NY: The Guildford Press

Dechesne, M.; Janssen, J. and van Knippenberg, A. (2000). Derogation and Distancing as Terror-Management Strategies: The Moderating Role of Need for Closure and Permeability of Group Boundaries. Journal of Personality and Social Psychology, vol. 79, no. 6, pp. 923-932 
Dechesne, M.; Pyszczynski, T.; Arndt, J.; Random, S.; Sheldon, K.; van Knippenberg, A. and Janssen, J. (2003). Literal and Symbolic Immortality: The Effect of Evidence of Literal Immortality on Self-Esteem in Response to Mortality Salience. Journal of Personality and Social Psychology, vol. 84, no. 4, pp. 722-737

Deci, E. and Ryan, R. (1985). Intrinsic Motivation and Self-Determination in Human Behaviour. New York, NY: Plenum

Deci, E. Ryan, R. (2006). Hedonia, Eudaimonia, and Well-being: An Introduction. Journal of Happiness Studies, vol. 9 , no. 1, pp. 1-11

Deci, E. and Ryan, R. (2000). The "What" and "Why" of Goal Pursuits: Human Needs and the Self-Determination of Behavior. Psychological Inquiry, vol. 11, no. 4, pp. 227-268

Deci, E.; Koestner, R. and Ryan, R. (1999). A Meta-Analytic Review of Experiments Examining the Effects of Extrinsic Rewards on Intrinsic Motivation. Psychological Bulletin, vol. 125, no. 6, pp. 627-668

Deci, E.; Olafsen, A. and Ryan, R. (2017). Self-Determination Theory in Work Organizations: The State of a Science. Annual Review of Organizational Psychology and Organizational Behaviour, vol. 4, no. 1, pp. 19-43

Deci, E.; Ryan, R.; Gagné, M.; Leone, D.; Usunov, J. and Kornazheva, B. (2001). Need Satisfaction, Motivation, and Well-Being in the Work Organizations of a Former Eastern Bloc Country: A Cross-Cultural Study of SelfDetermination. Personality and Social Psychology Bulletin, vol. 27, no. 9, pp. 930-942

Diener, E.; Wirtz, D.; Tov, W.; Kim-Prieto, C.; Choi, D.; Oishi, S. and Biswas-Diener, R. (2010). New Well-Being Measures: Short Scales to Assess Flourishing and Positive and Negative Feelings. Social Indicators Research, vol. 87 , no. 2 , pp. 143-156

De Neve, J. and Oswald, A. (2012). Estimating the Effects of Life Satisfaction and Positive Affect on Later Outcomes Using Sibling Data. Proceedings of the National Academies of Sciences USA, vol. 109, no. 49, pp. 19953-19958

De Neve, J.; Diener, E.; Tay, L. and Xuereb, C. (2013). The Objective Benefits of Subjective Well-Being. In J. Helliwell, R. Layard and J. Sachs (eds.), World Happiness Report II, pp. 54-74. New York, NY: Earth Institute, Columbia University

De Neve, J.; Ward, G.; De Keulenaer, F.; van Landeghem, B.; Kavetsos, G. and Norton, M. (2018). The Assymetric Experience of Positive and Negative Economic Growth: Global Evidence Using Subjective Well-Being Data. Review of Economics and Statistics, vol. 100, no. 2, pp. 362-375

DeNeve, K. and Cooper, H. (1998). The Happy Personality: A Meta-Analysis of 137 Personality Traits and Subjective Well-Being. Psychological Bulletin, vol. 124, no. 2, pp. 197-229

Devine, J. (2002). Ethnography of a Policy Process: A Case Study of Land Redistribution in Bangladesh. Public Administration and Development, vol. 22, no. 5, pp. 403-422

Devine, J.; Camfield, L. and Gough, I. (2008). Autonomy or Dependence-or Both? Perspectives from Bangladesh. Journal of Happiness Studies, vol. 9, no. 1, pp. 105-138

Devos, T.; Hyunh, Q. and Banaji, M. (2012). Implicit Self and Identity. In M. Leary and J. Tangney (eds.), Handbook of Self and Identity: Second Edition, pp. 155-179.

Diener, E. and Lucas, R. (1999). Personality and Subjective Well-Being. In D. Kahneman, E. Diener and R. Schwarz (eds.), Well-Being: The Foundations of Hedonic Psychology, pp. 213-229. New York, NY: Russell Sage.

Diener, E. and Seligman, M (2004). Beyond Money: Toward an Economy of Well-Being. Psychological Science in the Public Interest, vol. 5, no. 1, pp. 1-31

Diener, E. and Biswas-Diener, R. (2008). Happiness: Unlocking the Mysteries of Psychological Wealth. Malden, MA: Blackwell

Diener, E.; Suh, E.; Lucas, R. and Smith, H. (1999). Subjective Well-Being: Three Decades of Progress. Psychological Bulletin, vol. 125, no. 2, pp. 276-302

Diener, E. Lucas, R. and Scollon, C. (2006). Beyond the Hedonic Treadmill: Revisiting the Adaptation Theory of Wellbeing. American Psychology, vol. 61, no. 4, pp. 305-314

Diener, E.; Lucas, R.; Schimmack, W. and Helliwell, R. (2009). Well-Being for Public Policy. Oxford, UK: Oxford University Press

Diener, E.; Wirtz, D.; Tov, W.; Kim-Prieto, C.; Choi, D.; Oishi, S. and Biswas-Diener, R. (2010). New Well-Being Measures: Short Scales to Assess Flourishing and Positive and Negative Feelings. Social Indicators Research, vol. 87, no. 2, pp. 143-156

Diener, E.; Inglehart, R. and Tay, L. (2013). Theory and Validity of Life Satisfaction Scales. Social Indicators Research, vol. 112, no. 3, pp. 497-527

Dixon, T. and Baumeister, R. (1991), Escaping the Self: The Moderating Effect of Self-Complexity. Personality and Social Psychology Bulletin, vol. 17, no. 4, pp. 363-368 
Dolan, P.; Peasgood, T. and White, M. (2008). Do We Really Know What Makes Us Happy? A Review of the Economic Literature on the Factors Associated with Subjective Well-Being. Journal of Economic Psychology, vol. 29, no. 1, pp. 94-122

Do, A.; Rupert, A. and Wolford, G. (2008). Evaluations of Pleasurable Experiences: The Peak-End Rule. Psychonomic Bulletin and Review, vol. 15, no. 1, pp. 96-98 Dolan, P. (2014). Happiness by Design. London, UK: Penguin

Doris, J. (2002). Lack of Character: Personality and Moral Behaviour. New York, NY: Cambridge University Press.

Dorjee, D. (2014). Mind, Brain and the Path to Happiness: A Guide to Buddhist Mind Training and the Neuroscience of Happiness. London, UK: Routledge

Dyrdal, G. and Lucas R. (2013). Reaction and Adaptation to the Birth of a Child: A Couple-Level Analysis. Developmental Psychology, vol. 49, no. 4, pp. 749-761

Dunn, E.; Aknin, L. and Norton, M. (2008). Spending Money on Others Promotes Happiness. Science, vol. 319 , no. 5870, pp. 1687-1688

Durie, M. (2006). Measuring Maori Wellbeing. New Zealand Treasury Guest Lecture Series. Wellington, NZ: Treasury.

Easterlin, R. (1974). Does Economic Growth Improve the Human Lot? Some Empirical Evidence. In David and Reder eds. Nations and households in economic growth: essays in honour of Moses Abramovitz, New York, NY: Academic Press

Easterlin, R.; Wang, F. and Wang, S. (2017). Growth and Happiness in China, 1990-2015. In J. Helliwell, R. Layard and J. Sachs (eds.), World Happiness Report, ch. 3, pp. 48-83

Ellison, C. (1991). Religious Involvement and Subjective Well-Being. Journal of Health and Social Behaviour, vol. 32, no. 1, pp. 80-99

Emmons, R. (1986). Personal Strivings: An Approach to Personality and Subjective Well-Being. Journal of Personality and Social Psychology, vol. 51, no. 5, pp. 1058-1068

Emmons, R. (1999). The Psychology of Ultimate Concerns: Motivation and Spirituality in Personality. New York, NY: Guildford

Emmons, R. (2008). Thanks! How Practicing Gratitude Can make you Happier. New York: Mariner

Emmons, R. and McCullough, M. (2003). Counting Blessings versus Burdens: An Experimental Investigation of Gratitude and Subjective Well-Being in Daily Life. Journal of Personality and Social Psychology, vol. 84, no. 2, pp. 377-389

Emmons, R.; Cheung, C. and Tehrani, K. (1998). Assessing Spirituality through Personal Goals: Implications for Research on Religion and Subjective Well-Being. Social Indicators Research, vol. 45, no. 1-3, pp. 391-422

Ernest-Jones, M.; Nettle, D. and Bateson, M. (2011). Effects of Eye Images on Everyday Cooperative Behaviour: A Field Experiment. Evolution and Human Behaviour, vol. 32, no. 2, pp. 172-178

Fabian, M. (2018). The Ends and Means of Public Policy. In M. Fabian and R. Breunig (eds.), Hybrid Public Policy Innovations: Contemporary Policy Beyond Ideology, pp. 3-27. London, UK: Routledge.

Fabian, M. (2018). Racing from Subjective Well-Being to Public Policy: A Review of The Origins of Happiness. Journal of Happiness Studies, online first.

Fader, J. (2016). Life as Sport: What Top Athletes Can Teach You About How to Win in Life. Boston, MA: Da Capo

Favara, M. and Sanchez, A. (2017). Psychosocial Competencies and Risky Behaviour in Peru. IZA Journal of Labor and Development, vol. 6, no. 3, pp. 1-40

Feldman, F. (2002). The Good Life: A Defence of Attitudinal Hedonism. Philosophy and phenomenological research, vol. 65, no. 3, pp. 604-628

Ferrer-i-Carbonell, A. and Fritjers, P. (2004). How important is Methodology for the Estimates of the Determinants of Happiness? The Economic Journal, vol. 114, no. 497, pp. 641-659

Fivush, R. and Haden, C. (eds.) (2003). Autobiographical Memory and the Construction of a Narrative Self. Mahwah, NJ: Erlbaum

Fletcher, G. (ed.), (2015). The Routledge Handbook of Philosophy of Well-Being. London, UK: Routledge.

Fleurbaey, M. and Maniquet, F. (2011). A Theory of Fairness and Social Welfare. Cambridge, UK: Cambridge University Press.

Fleurbaey, M. and Blanchet, D. (2013). Beyond GDP: Measuring Welfare and Assessing Sustainability. Oxford, UK: Oxford University Press

Foster-McGregor, N. and Verspagen, B. (2016). The Role of Structural Change in the Economic Development of Asian Economies. Asian Development Review, vol. 33, no. 2, pp. 74-93

Frankl. V. (1946/2008). Man's Search for Meaning. London, UK: Rider 
Frankl, V. (1969). The Will to Meaning: Foundations and Applications of Logotherapy. New York, NY: Meridian Frankl. V. (1975). The Unconscious God. New York, NY: Pocket Books

Frankl. V. (1978). The Unheard Cry for Meaning: Psychotherapy and Humanism. New York, NY: Touchstone Books

Frankl. V. (2000). Recollections: An Autobiography. Trans. J. Fabry and J. Fabry. New York, NY: Perseus Books Group

Frankl. V. (2010). The Feeling of Meaninglessness: A Challenge to Psychotherapy and Philosophy. Milwaukee, WI: Marquette University Press

Frattaroli, J. (2006). Experimental Disclosure and its Moderators: a Meta-Analysis. Psychological Bulletin, vol. 132, no. 6, pp. 823-865

Frederick, S. and Loewenstein, G. (1999). Hedonic Adaptation. In D. Kahneman, N. Schwartz and E. Diener (eds.), Well-Being: The Foundations of Hedonic Psychology, pp. 302-329. New York, NY: Russell Sage.

Freitas, A.; Salovey, P and Liberman, N. (2001). Abstract and Concrete Self-Evaluative Goals. Journal of Personality and Social Psychology, vol. 80, no. 3, pp. 410-424

Frey, B. and Stutzer, A. (2002). Happiness and Economics: How the Economy and Institutions Affect Wellbeing. Princeton, NJ: Princeton University Press.

Frey, B. and Stutzer, A. (2008). Stress That Doesn’t Pay: The Commuting Paradox. The Scandinavian Journal of Economics, vol. 110, no. 2, pp. 339-366

Frederickson, B. (2000). Extracting Meaning from Past Affective Experiences: The Importance of Peaks, Ends and Specific Emotions. Cognition and Emotion, vol. 14, no. 4, pp. 577-606

Frederickson, B.; Cohn, M.; Coffey, K.; Pek, J. and Finkel, S. (2008). Open Hearts Build Lives: Positive Emotions, Induced through Loving-Kindness Meditation Build Consequential Personal Resources. Journal of Personality and Social Psychology, vol. 95, pp. 1045-1062

Frijda, N. (1986). The Emotions. New York, NY: Cambridge University Press; Paris, FR: Editions de la Maison des Sciences de l'Homme.

Frijda, N. (1999). Emotions and Hedonic Experience. In D. Kahneman, E. Diener and N. Schwartz (eds.), Wellbeing: The Foundations of Hedonic Psychology, pp. 190-210. New York, NY: Russell Sage Foundation

Fritjers, P. and Mujcic, R. (2013). Economic Choices and Status: Measuring Preferences for Income Rank. Oxford Economic Papers, vol. 65, no. 1, pp. 47-73

Fritz, M.; Walsh, L. and Lyubomirsky, S. (2017). Staying Happier. In M. Robertson and M. Eid (eds.), The Happy Mind, pp. 95-114. Cham, CH: Springer International Publishing. Fromm, E. (1941/1994). Escape from Freedom. New York, NY: Holt Paperbacks

Fujiwara, D. and Dolan, P. (2016). Happiness-Based Policy Analysis. In M. Adler and M. Fleurbaey (eds.), The Oxford Handbook of Well-Being and Public Policy, pp. 286-320. Oxford, UK: Oxford University Press.

Ganesharajah, C. (2009). Indigenous Health and Wellbeing: The Importance of Country. Native Title Research Report No. 1/2009. Canberra, AU: Australian Institute of Aboriginal and Torres-Strait Islander Studies AIATSIS.

Garfield, C. (1973). A Psychometric and Clinical Investigation of Frankl's Concept of Existential Vacuum and Anomie. Psychiatry, vol. 36, no. 4, pp. 396-408

Gere, J.; Schimmack, U.; Pinkus, R. and Lockwood, P. (2011). The Effects of Romantic Partners' Goal Congruence on Affective Well-Being. Journal of Research in Personality, vol. 45, no. 6, pp. 549-559.

Gelfand, M.; Raver, J.; Nishii, L.; Leslie, L.; Lun, J.; Lim, B.; Duan, L.; Almaliach, A.; Ang, S.; Arnadottir, J.; Aycan, Z.; Boehnke, K.; Boski, P.; Cabecinhas, R.; Chan, D.; Chhokar, J.; D’Amato, A.; Ferrer, M.; Fischlmayr, I.; Fischer, R.; Fülöp, M.; Georgas, J.; Kashima, E.; Kashima, Y.; Kim, K.; Lempereur, A.; Marquez, P.; Othman, R.; Overlaet, B.; Panagiotopoulou, P.; Peltzer, K.; Perez-Florizno, L.; Ponomarenko, L.; Realo, A.; Schei, V.; Schmitt, M.; Smith, P.; Soomro, N.; Szabo, E.; Taveesin, N.; Toyama, M.; Van de Vliert, E.; Vohra, N.; Ward, C. and Yamaguchi, S. (2011). Differences Between Tight and Loose Cultures: A 33 Nation Study. Science, vol. 332, no. 6033, pp. 1100-1104

Gilbert, P.; McEwan, K.; Matos, M. and Rivis, A. (2011). Fears of Compassion: Development of Three Self-Report Measures. Psychology and Psychotherapy: Theory, Research and Practice, vol. 84, no. 1, pp. 239-255

Glaeser, E. (2012). Triumph of the City: How Our Greatest Invention Makes Us Richer, Smarter, Greener, Healthier and Happier. New York, NY: Penguin

Gonnerman, M.; Parker, C.; Lavine, H. and Huff, J. (2000). The relationship Between Self-Discrepancies and Affective States: The Moderating Roles of Self-Monitoring and Standpoints on the Self. Personality and Social Psychology Bulletin, vol. 26, no. 7, pp. 810-819 
Graham, C. (2011). The Pursuit of Happiness: An economy of Well-Being. Washington, DC: Brookings Institution Press.

Graham, C. (2011). Adaptation amidst Prosperity and Adversity: Some Insights from Happiness around the World. World Bank Research Observer, vol. 26, no. 1, pp. 105-137

Graham, C. (2012). Happiness around the World: The Paradox of Happy Peasants and Miserable Millionaires. Oxford, UK: Oxford University Press

Graham, C. (2017). Happiness for All? Unequal Hopes and Lives in Pursuit of the American Dream. Princeton, NJ: Princeton University Press

Graham, C. and Pettinato, S. (2002). Happiness and Hardship: Opportunity and Insecurity in New Market Economies. Washington, DC: Brookings Institution Press

Graham, C. and Lora, E. (2009). Paradox and Perception: Measuring Quality of Life in Latin America. Washington, DC: Brookings Institution Press

Graham, C. and Nikolova, M. (2015). Bentham or Aristotle in the Development Process? An Empirical Investigation of Capabilities and Subjective Well-Being around the World. World Development, vol. 68, no. 1, pp. $163-179$

Graham, C.; Chattopadhyay, S. and Picon, M. (2010). Adapting to Adversity: Happiness and the 2009 Economic Crisis in the United States. Social Research, vol. 77, no. 2, pp. 715-748

Greenberg, J.; Koole, S. and Pyszczynski, T. (eds.). Handbook of Experimental Existential Psychology. New York, NY: Guildford Press

Greene, J. (2014). Moral Tribes: Emotion, Reason and the Gap between Us and Them. London, UK: Atlantic Books

Gregory, A. (2015). Hedonism. In G. Fletcher (ed.), Routledge Handbook of Philosophy of Well-Being, pp. 113123. London, UK: Routledge.

Griffin, J. (1986). Well-being: Its Meaning, Measurement and Moral Importance. Oxford, UK: Clarendon

Haidt, J. (2001). The Emotional Dog and its Rational Tail. Psychological Review, vol. 108, no. 4, pp. 814-834.

Haidt, J. (2012). The Righteous Mind: Why Good People are divided by Politics and Religion. New York, NY: Penguin

Haidt, J. and Algoe, S. (2004). Moral Amplification and the Emotions that attach us to Saints and Demons. In J. Greenberg, S. Koole and T. Pyszczynski (eds.), Handbook of Existential Psychology, pp. 322-335. New York: Guildford

Haley-Jones, K. and Fessler, D. (2005). Nobody's Watching? Subtle Cues Affect Generosity in an Anonymous Economic Game. Evolution and Human Behaviour, vol. 26, no. 3, pp. 245-256

Hanson, R. (2013). Hardwiring Happiness: How to Reshape Your Brain and Your Life. London, UK: Ryder.

Hanushek, E. (1986). The Economics of Schooling: Production and Efficiency in Public Schools. Journal of Economic Literature, Vol. 24, No. 3, pp. 1141-1177

Harari, Y. (2011). Sapiens: A Brief History of Humankind. New York, NY: Harper

Harter, S. (1999). The Construction of the Self: 2nd edition. New York, NY: Guildford

Harter, S. (2012). Emerging Self-Processes during Childhood and Adolescence. In M. Leary and J. Tangney (eds.), Handbook of Self and Identity: Second Edition, pp. 680-715. New York, NY: Guildford

Harter, S.; Bresnick, S.; Bouchey, H. and Whitesell, N. (1997). The Development of Multiple Role-Related Selves during Adolescence. Development and Psychopathology, vol. 9, no. 4, pp. 835-854

Haybron, D. (2001). Happiness and Pleasure. Philosophy and Phenomenological Research, vol. 62, no. 3, pp. 501528

Haybron, D. (2008). The Pursuit of Unhappiness: The Elusive Psychology of Well-Being. Oxford, UK: Oxford University Press

Haybron, D. and Tiberius, V. (2015). Well-Being Policy: What Standard of Well-Being? Journal of the American Philosophical Association, vol. 1, no. 4, pp. 712-733

Heady, B. (2010). The Set-Point Theory of Well-Being has Serious Flaws: On the Eve of a Scientific Revolution? Social Indicators Research, vol. 97, no. 1, pp. 7-21

Heady, B.; Muffels, R. and Wagner, G. (2014). National Panel Studies Show Substantial Minorities Recording Long-Term Change in Life Satisfaction: Implications for Set Point Theory. In K. Sheldon and R. Lucas (eds.), Stability of Happiness: Theories and Evidence on Whether Happiness can Change, pp. 101-121. New York: Academic Press

Heathwood, C. (2015). Desire Fulfilment Theory. In G. Fletcher (ed.), Routledge Handbook of Philosophy of WellBeing, pp. 135-147. London, UK: Routledge.

Heidegger, M. (1923/1962). Being and Time. trans. J. Macquarie and E. Robinson. Malden: MA: Blackwell 
Hektner, J.; Schmidt, J. and Csikszentmihaly, M. (2007). Experience Sampling Method: Measuring the Quality of Everyday Life. Newbury Park, CA, NY: Sage

Helliwell, J., Layard, R. and Sachs, J. (2017). The World Happiness Report 2017. New York, NY: Sustainable Development Solutions Network.

Helliwell, J., Layard, R. and Sachs, J. (2018). The World Happiness Report 2018. New York, NY: Sustainable Development Solutions Network.

Heppen, J. and Ogilvie, D. (2003). Predicting Affect from Global Self-Discrepancies: The Dual Role of the Undesired Self. Journal of Social and Clinical Psychology, vol. 22, no. 4, pp. 347-368

Higgins, T. (1987). Self-Discrepancy Theory: A Theory Relating Self and Affect. Psychological Review, vol. 94 no. 3 pp. $319-340$

Higgins, T. (1991). Development of Self-Regulatory and Self-Evaluative Processes: Costs, Benefits and Tradeoffs. In M. Gunnar and A. Sroufe (eds.), Self-Processes and Development: The Minnesota Symposium on Child Development, vol. 23. pp. 125-166. Hillsdale, NJ: Erlbaum

Hindriks, F. and Douven, I. (2018). Nozick’s Experience Machine: An Empirical Study. Philosophical Psychology, vol. 31, no. 2, pp. 278-298

Hirai, T. (2017). The Creation of the Human Development Approach. Basingstoke, UK: Palgrave MacMillan

Hixon, J. and Swann, W. (1993). When Does Introspection Bear Fruit? Self-Reflection, Self-Insight and Interpersonal Choices. Journal of Personality and Social Psychology, vol. 64, no. 1, pp. 34-43

Howell, R.; Chenot, D.; Hill, G. and Howell, C. (2011). Momentary Happiness: The Role of Psychological Need Satisfaction. Journal of Happiness Studies, vol. 12, no. 1, pp. 1-15

Huppert, F. A. and So, T. T. C. (2013). Flourishing Across Europe: Application of a New Conceptual Framework for Defining Well-Being. Social Indicators Research, vol. 110, no. 3, pp. 837-861

Husser, J. and Fernandez, K. (2018). We are Happier than We Realize: Underestimation and Conflation in Measuring Happiness. Journal of Happiness Studies, vol. 19, no. 2, pp. 587-606

Hutz, C.; Midgett, A.; Pacico, J.; Bastianello, M. and Zanon, C. (2014). The Relationship of Hope, Optimism, SelfEsteem, Subjective Well-Being, and Personality in Brazilians and Americans. Psychology, vol. 5, no. 6, pp. 514-522

Ilardi, B.; Leone, D.; Kasser, T. and Ryan, R. (1993). Employee and Supervisor Ratings of Motivation: Main Effects and Discrepancies Associated with Job Satisfaction and Adjustment in a Factory Setting. Journal of Applied Social Psychology, vol. 23, no. 21, pp. 1789-1805

Inferna, F. and Wiest, M. (2018). The Effect of Disability Onset across Adult Life Span. The Journals of Gerontology, vol. 73, no. 5, pp. 755-766.

Iyengar, S. and Lepper, M. (1999). Rethinking the Value of Choice: A Cultural Perspective on Intrinsic Motivation. Journal of Personality and Social Psychology, vol. 76 no. 3 pp. 349-366

Jacobs-Bao, K and Lyubomirsky, S. (2013). The Rewards of Happiness. In I. Boniwell, S. David and C. Ayers (eds.), Oxford Handbook of Happiness, pp. 119-133. Oxford, UK: University Press. James, W. (1890). Principles of Psychology. Chicago. MI: Encyclopaedia Britannica

Jang, H.; Reeve, J.; Ryan, R. and Kim, A. (2009). Can Self-Determination Theory Explain What Underlies the Productive, Satisfying Learning Experiences of Collectivistically Oriented Korean Students? Journal of Educational Psychology, vol. 101, no. 3, pp. 644-661

Jang, H.; Reeve, J. and Deci, E. (2010). Engaging Students in Learning Activities: It Is Not Autonomy Support or Structure, but Autonomy Support and Structure. Journal of Educational Psychology, vol. 102, no. 3, pp. 588600

Jazaieri, H.; Jinpa, G.; McGonigal, K.; Rosenberg, E.; Finkelstein, J.; Simon-Thomas, E.; Cullen, M.; Doty, J.; Gross, J. and Goldin, P. (2013). Enhancing Compassion: A Randomised Controlled Trial of a Compassion Cultivation Program. Journal of Happiness Studies, vol. 14, no. 4, pp. 1113-1126

Johnson, E.; Vincent, N. and Ross, L. (1997). Self-Deception versus Self-Esteem in Buffering the Negative Effects of Failure. Journal of Research in Personality, vol. 31, no. 3, pp. 385-405

Jung, C. (1928/2017). The Spiritual Problem of Modern Man. Trans. W. Dell and C. Baynes in Modern Man in Search of a Soul, pp. 226-254. Eastford, CT: Martino Fine Books

Jupp, E.; Pykett, J. and Smith, F. (eds.), (2016). Emotional States: Sites and Spaces of Affective Governance. London, UK: Routledge

Kabat-Zinn, J. (2006). Mindfulness-Based Interventions in Context: Past, Present and Future. Clinical Psychology Science and Practice, vol. 10, no. 2, pp. 144-156

Kagan, S. (1984). Me and My Life. Proceedings of the Aristotelian Society, vol. 94, pp. 309-324 
Kahneman, D. (1999). Objective Happiness. In D. Kahneman, E. Diener and N. Schwartz (eds.), Wellbeing: the foundations of hedonic psychology, pp. 3-25. New York, NY: Russell Sage Foundation

Kahneman, D. (2010). Comment: This Is Not How Science Is Done. Judgement and Decision Making, vol. 5, no. 6, pp. $458-466$

Kahneman, D. (2011). Thinking, Fast and Slow. New York, NY: Penguin

Kahneman, D. and Tversky, A. (1979). Prospect Theory: An Analysis of Decision under Risk. Econometrica, vol. 47, no. 2, pp. 263-291

Kahneman, D. and Krueger, A. (2006). Developments in the Measurement of Subjective Well-Being. Journal of Economic Perspectives, vol. 20, no. 1, pp. 3-24

Kahneman, D. and Deaton, A. (2010). High Income Improves Evaluation of Life But Not Emotional Well-Being. Proceedings of the National Academies of Sciences USA, vol. 107, no. 38, pp. 16489-16493.

Kahneman, D.; Frederickson, B.; Schreiber, C. and Redelmeier, D. (1993). When More Pain is preferred to Less: Adding a Better End. Psychological Science, vol. 4, no. 6, pp. 401-405

Kahneman, D.; Diener, E. and Schwartz, N. (1999). Preface. In D. Kahneman, E. Diener and N. Schwartz (eds.), Wellbeing: The Foundations of Hedonic Psychology, pp. ix-xii, New York, NY: Russell Sage Foundation

Kahneman, D.; Diener, E. and Schwarz, N. (1999). Wellbeing: The Foundations of Hedonic Psychology. New York, NY: Russell Sage Foundation

Kahneman, D.; Krueger, A.; Schkade, D.; Schwarz, N. and Stone, A. (2004). Toward National Well-Being Accounts. American Economic Review, vol. 94, pp. 429-434

Kahneman, D.; Krueger, A.; Schkade, D.; Schwarz, N. and Stone, A. (2006). A Survey Method for Characterizing Daily Life Experience: The Day Reconstruction Method. Science, vol. 306, no. 5702, pp. 1776-1780

Kahneman, D.; Krueger, A.; Schkade, D.; Schwarz, N. and Stone, A. (2006). Would You Be Happier if you were Richer? A Focusing Illusion. Science, vol. 30, no. 5782, pp. 1908-1910

Kaminitz, S. (2018). Happiness Studies and the Problem of Interpersonal Comparisons of Satisfaction: Two Histories, Three Approaches. Journal of Happiness Studies, vol. 19, no. 2, pp. 423-442

Kapteyn, A.; Smith, J. and Van Soest, A. (2013). Are Americans Really Less Happy With Their Incomes? Review of Income and Wealth, vol. 59, no. 1, pp. 44-65

Kashdan, T. B., Biswas-Diener, R. and King, L. A. (2008). Reconsidering Happiness: The Costs of Distinguishing Between Hedonics and Eudaimonia. The Journal of Positive Psychology, vol. 3, no. 4, pp. 219-233

Kashdan, T. B., Biswas-Diener, R. and King, L. A. (2009). Two Traditions of Wellbeing Research, Not Two Distinct Types of Wellbeing. The Journal of Positive Psychology, vol. 4, no. 3, pg. 208-211. Kasser, T. (2003). The High Price of Materialism. Cambridge, MA: MIT Press

Kasser, T. and Ryan, R. (1993). A Dark Side of the American Dream: Correlates of Financial Success as a Central Life Aspiration. Journal of Personality and Social Psychology, vol. 65, no. 2, pp. 410-422

Kasser, T. and Ryan, R. (1996). Further Examining the American Dream: Differential Correlates of Intrinsic and Extrinsic Goals. Personality and Social Psychology Bulletin, vol. 22, no. 3, pp. 280-287

Kasser, T. and Ryan, R. (2001). Be Careful What You Wish For: Optimal Functioning and the Relative Attainment of Intrinsic and Extrinsic Goals. In P. Schmuck and K. Sheldon (eds.), Life Goals and Well-Being: Towards a Positive Psychology of Human Striving, pp. 116 - 131. Ashland, OH: Hogrefe and Huber.

Kasser, T. and Ahuvia, A. (2002). Materialistic Values and Well-Being in Business Students. European Journal of Social Psychology, vol. 32, no. 1, pp. 137-146

Keng, S.; Smoski, M. and Robbins, C. (2011). Effects of Mindfulness on Psychological Health: A Review of Empirical Studies. Clinical Psychology Review, vol. 31, no. 6, pp. 1041-1056

Keyes, C.; Schmotkin, D. and Ryff, C. (2002). Optimizing Well-Being: The Empirical Encounter of Two Traditions. Journal of Personality and Social Psychology, vol. 82, no. 6, pp. 179-196

Kierkegaard, S. (1843/2005). Fear and Trembling. Trans. A. Hannay. London, UK: Penguin

Kierkegaard, S. (1849/2008). The Sickness unto Death. Trans. A. Hannay. London, UK: Penguin

King, L. and Hicks, J. (2012). Positive Affect and Meaning in Life: The Intersection of Hedonism and Eudaimonia. In P. Wong (ed.), The Human Quest for Meaning: Theories, Research and Applications, $2^{\text {nd }}$ edition, pp. 125142. London, UK: Routledge

King, L.; Hicks, J.; Krull, J. and Del Gaiso, A. (2006). Positive Affect and the Experience of Meaning in Life. Journal of Personality and Social Psychology, vol. 90, no. 1, pp. 179-196

Knabe, A. and Rätzel, S. (2011). Scarring or Scaring? The Psychological Impact of Past Unemployment and Future Employment Risk. Economica, vol. 78, no. 31, pp. 283-293 
King, L. and Hicks, J. (2012). Positive Affect and Meaning in Life: The Intersection of Hedonism and Eudaimonia. In Wong, V. (ed.), The Quest for Human Meaning: Theories, Research and Application-Second Edition, pp. 125-142. London, UK: Routledge

Knight, J. and Gunatilaka, R. Subjective Well-Being and Social Evaluation: A Case Study of China. In A. Clark and C. Senik, (2014). Happiness and economic growth, pp. 179-215. Oxford, UK: Oxford University Press

Kraut, R. (1979). Two Conceptions of Happiness. Philosophical Review, vol. 88, no. 2, pp. 167-197 Kraut, R. (1989). Aristotle on the Human Good. Princeton, NJ: Princeton University Press

Kring, A. (2008). Emotion Disturbances as Transdiagnostic Processes in Psychopathology. In M. Lewis, J. Haviland-Jones and L. Feldman-Barrett (eds.), Handbook of Emotions: Third edition, pp. 691-708. New York, NY: Guildford Press

Krueger, A. and Schkade, D. (2008). The Reliability of Subjective Well-Being Measures. Journal of Public Economics, vol. 92, no. 8-9, pp. 1833-1845

Kruger, J. and Dunning, D. (1999). Unskilled and Unaware of it: How Difficulties in Recognising One's Own Incompetence Lead to Inflated Self-Assessment. Journal of Personality and Social Psychology, vol. 77, no. 6, pp. 1121-1134

Kruse, E.; Chancellor, J.; Ruberton, P. and Lyubomirsky, S. (2014). An Upward Spiral between Gratitude and Humility. Social Psychological and Personality Science, vol. 5, no. 7, pp. 805-814

Kuhl, J. and Koole, S. (2004). Workings of the Will: A Functional Approach. In J. Greenberg, S. Koole and T. Pyszczynski (eds.), Handbook of Experimental Existential Psychology, pp. 431-448. New York, NY: Guildford

Kuppens, P; Realo, A. and Diener, E. (2008). The Role of Positive and Negative Emotions in Life Satisfaction Judgements across Nations. Journal of Personality and Social Psychology, vol. 95, no. 1, pp. 66-75

Lacey, H.; Loewenstein, G.; Riis, J.; Fagerlin, A.; Smith, D. and Ubel, P. (2008). Are They Really That Happy? Exploring Scale Norming in Estimates of Well-Being. Health Psychology, vol. 27, no. 6, pp. 669-675

La Guardia, J.; Ryan, R.; Couchman, C. and Deci, E. (2000). Within-Person Variation in Security of Attachment: A Self-Determination Theory Perspective on Attachment, Need Fulfilment and Well-Being. Journal of Personality and Social Psychology, vol. 79, no. 3, pp. 367-384

Laurin, J.; Joussemet, M.; Tremblay, R. and Boivin, M. (2015). Early Forms of Controlling Parenting and the Development of Childhood Anxiety. Journal of Child and Family Studies, vol. 24, no. 11, pp. 3279-3292 Layard, R. (2005). Happiness: Lessons from a New Science. New York, NY: Penguin

Leary, M. and Baumeister, R. (2000). The Nature and Function of Self-Esteem: Sociometer Theory. Advances in Experimental Social Psychology, vol. 32, no. 1, pp. 1-62

Leary, M. (2012). Sociometer Theory. In P. Van Lange, A. Kruglanski, and E. Higgins (eds.), Handbook of Theories of Social Psychology, (pp. 151-159). Thousand Oaks, CA: Sage Publications Ltd. LeBar, M. (2013). The Value of Living Well. Oxford, UK: Oxford University Press

Lerner, J. and Tetlock, P. (2003). Bridging Individual, Interpersonal and Institutional Approaches to Judgement and Decision Making: The Impact of Accountability on Cognitive Bias. In S. Schneider and J. Shanteau (eds.), Emerging Perspectives on Judgement and Decision Research, pp. 431-457. Cambridge, UK: Cambridge University Press.

Lewis, M. (2008). Self-Conscious Emotions: Embarrassment, Pride, Shame and guilt. In M. Lewis, J. HavilandJones and L. Feldman-Barrett (eds.), Handbook of Emotions: Third Edition, pp. 742-756. New York, NY: Guildford

Li, Y.; Guan, D.; Tao, S.; Wang, X. and He, K. (2018). A Review of Air Pollution Impact on Subjective WellBeing: Survey versus Visual Psychophysics. Journal of Cleaner Production, vol. 184, pp. 959-968

Lickert, R. (1932). A Technique for the Measurement of Attitudes. Archives of Psychology, vol. 22, no. 1, pp. 5-55

Lim, C. and Putnam, R. (2010). Religion, Social Networks and Life Satisfaction. American Sociological Review, vol. 75, no. 6, pp. 914-933

Lindqvist, E.; Östling, R. and Cesarini, D. (2008). Long Run Effects of Lottery Wealth on Psychological WellBeing. IFN Working Paper No. 1220.

Linville, P. (1987). Self-Complexity as a Cognitive Buffer against Stress-Related Illness and Depression. Journal of Personality and Social Psychology, vol. 52, no. 4, pp. 663-676

Liu, Y.; Zhang, F.; Wu, F.; Liu, Y. and Li, Z. (2017). The Subjective Well-Being of Migrant in Guangzhou, China: The Impacts of the Social and Physical Environment. Cities, vol. 60, no. 1, pp. 333-342

Locke, E. and Latham, G. (1990). A Theory of Goal-Setting and Task Performance. Englewood Cliffs, NJ: PrenticeHall

Loewenstein, G. and Ubel, P. (2008). Hedonic Adaptation and the Role of Decision and Experience Utility in Public Policy. Journal of Public Economics, vol. 92, no. 8-9, pp. 1795-1810 
Lucas, R. (2016). Subjective Well-Being in Psychology. In M. Adler and M. Fleurbaey (eds.) The Oxford Handbook of Well-Being and Public Policy. Oxford, UK: Oxford University Press

Lucas, R. and Donellan, M. (2007). How Stable is Happiness? Using the STARTS Model to Estimate the Stability of Life Satisfaction. Journal of Research in Personality, vol. 41, no. 5, pp. 1091-1098.

Lucas, R., Diener, E. and Suh, E. (1996). Discriminant Validity of Well-Being Measures. Journal of Social and Personality Psychology, vol. 71, no. 3, pp. 616-628

Lucas, R.; Clark, A. Georgellis, Y. and Diener, E. (2004). Unemployment Alters the Set Point for Life Satisfaction. Psychological Science, vol. 15, no. 1, pp. 8-13

Lucas, R. and Diener, E. (2009). Personality and Subjective Wellbeing. In E. Diener (ed.) The Collected Works of Ed Diener. Social Indicators Research Series 37, pp. 75-102

Lucas, R.; Clark, A. E.; Georgellis, Y. and Diener, E. (2003). Re-Examining Adaptation and the Set Point Model of Happiness: Reactions to Changes in Marital Status. Journal of Personality and Social Psychology, vol. 84, pp. 527-539

Lucas, R. (2007). Long-Term Disability Is Associated with Lasting Changes in Subjective Well-Being: Evidence from two Nationally Representative Longitudinal Studies. Journal of Personality and Social Psychology, vol. 92, no. 4, pp. 717-730

Luhmann, M.; Hoffman, W.; Eid, M. and Lucas, R. (2012). Subjective Well-Being and Adaptation to Life Events: A Meta-Analysis. Journal of Personality and Social Psychology, vol. 102, no. 3, pp. 592-615.

Luyckx, K; Goosens, L.; Soenens, B.; Beyers, W. and Vansteenkiste, M. (2005). Identity Statuses Based Upon Four Rather Than Two Identity Dimensions: Extending and Refining Marcia's Paradigm. Journal of Youth and Adolescence, vol. 34, no. 6, pp. 605-618

Luyckx, K.; Schwartz, S.; Berzonsky, M.; Soenens, B.; Vansteenkiste, M.; Smits, I. and Goossens, L. (2008). Capturing Ruminative Exploration: Extending the Four-Dimensional Model of Identity Formation in Late Adolescence. Journal of Research in Psychology, vol. 42, no. 1, pp. 58-82

Lykken, D. (1999). Happiness: What Studies in Twins Show us About Nature, Nurture and the Happiness Set-Point. New York, NY: Golden Books.

Lykken, D. and Tellegen, A. (1996). Happiness is a Stochastic Phenomenon. Psychological Science, vol. 7, no. 3, pp. 186-189

Lynch, M.; La Guardia, J. and Ryan, R. (2009). On Being Yourself in Different Cultures: Ideal and Actual SelfConcept, Autonomy Support, and Well-Being in China, Russia, and the United States. Journal of Positive Psychology, vol. 4, no. 4, pp. 290-304

Lyubomirsky, S. and Ross, L. (1999). Changes in Attractiveness of Elected, Rejected and Precluded Alternatives: A Comparison of Happy and Unhappy Individuals. Journal of Personality and Social Psychology, vol. 73, pp. 1141-1157

Lyubomirsky, S.; Sousa, L. and Dickerhoof, R. (2006). The Costs and Benefits of Writing, Talking and Thinking about Life's Triumphs and Defeats. Journal of Personality and Psychology, vol. 90, no. 4, pp. 692-708

Lyubomirsky, S.; Boehm, J.; Kasri, F. and Zehm, K. (2011). The Cognitive and Hedonic Costs of Dwelling on Achievement-Related Negative Experiences: Implications for Enduring Happiness and Unhappiness. Emotion, vol. 11, no. 5, pp. 1152-1167

Marcus, G. (2004). The Birth of the Mind: How a Tiny Number of Genes Create the Complexity of Human Thought. New York, NY: Basic Books

Markus, H. and Kitayama, S. (2003). Models of Agency: Sociocultural Diversity in the Construction of Action. In V. Murphy-Berman and J. Berman (eds.), Nebraska Symposium on Motivation, vol. 49, pp. 1-57. Lincoln, NE: University of Nebraska Press.

Martin, L. Campbell, W. and Henry, C. (2004). The Roar of Awakening: Mortality Acknowledgement as a Call to Authentic Living. In J. Greenberg, S. Koole and T. Pyszczynski (eds.), Handbook of Existential Psychology, pp. 431-448. New York: Guildford

Masood, E. (2016). The Great Invention: The Story of GDP and the Making and Unmaking of the Modern World. London, UK: Pegasus Books

Matthews, G. (2008). Finding and Keeping Purpose in Life: Wellbeing and Ikigai in Japan and Elsewhere. In G. Matthews and C. Izquierdo (eds.), Pursuits of Happiness: Well-being in Anthropological Perspective. New York, NY: Berghahn Books

McAdams, D. (1993). The Stories we live By: Personal Myths and the Making of the Self. New York, NY: William Morrow \& Co.

McAdams, D. and Janis, L. (2004). Narrative Identity and Narrative Therapy. In L. Angus and J. McLeod, The Handbook of Narrative and Psychotherapy, pp. 158 - 171, Thousand Oaks, CA: Sage 
McAdams, D. and McLean, K. (2013). Narrative Identity. Current Directions in Psychological Science, vol. 22, no. 3, pp. 233-238

McClimans, L and Browne, J. (2011). Choosing a Patient-Reported Outcome Measure. Theoretical Medicine and Bioethics, vol. 32, no. 1, pp. 47-60

McClimans, L., Bickenbach, J., Westerman, M. and Schwartz, C. (2012). Philosophical Perspectives on Response Shift. Quality of Life Research, vol. 22, no. 7, pp. 1871-1878

McConnell, A.; Strain, L.; Brown, C. and Rydell, J. (2009). The Simple Life: On the Benefits of Low SelfComplexity. Personality Psychology Bulletin, vol. 35, no. 7, pp. 823-835

McCullough, M.; Kimeldorf, M. and Cohen, A. (2008). An Adaptation for Altruism? The Social Causes, Social Effects and Social Evolution of Gratitude. Current Directions in Psychological Science, vol. 17, no. 4, pp. 281285

McCullough, M.; Emmons, R. and Tsang, J. (2002). The Grateful Disposition: A Conceptual and Empirical Topography. Journal of Personality and Social Psychology, vol. 82, no. 1, pp. 112-127

McDonald, G. and Leary, M. (2012). Individual Differences in Self-Esteem. In M. Leary and J. Tangney (eds.), Handbook of Self and Identity: Second Edition, pp. 354-378. New York, NY: Guildford

McGregor, D.; Morelli, P; Matsuoko, J.; Rodenhurst, R.; Konh, N. and Spencer, M. (2003). An Ecological Model of Native Hawaiian Well-Being. Pacific Health Dialogue, vol. 10, no. 1, pp. 106-128

McGregor, I. and Holmes, J. (1999). How Storytelling Shapes Memory and Impressions of Relationship Events over Time. Journal of Personality and Social Psychology, vol. 76, no. 3, pp. 403-419

McGregor, I.; Zanna, M.; Holmes, J. and Spencer, S. (2001). Compensatory Conviction in the Face of Personal Uncertainty: Going to Extremes to be Oneself. Journal of Personality and Social Psychology, vol. 80, no. 3, pp. $838-852$

McGregor, I. (2003). Defensive Zeal: Compensatory Conviction about Attitudes, Values, Goals, Groups and SelfDefinition in the Face of Personal Uncertainty. In S. Spencer, S. Fein, M. Zanna and J. Olson (eds.), Motivated Social Perception: The Ontario Symposium, vol. 9, pp. 73-92. Mahwah, NJ: Erlbaum.

McGregor, I. and Marigold, D. (2003). Defensive Zeal and the Uncertain Self: What Makes You So Sure? Journal of Personality and Social Psychology, vol. 85, no. 5, pp. 838-852

McGregor, I. (2004). Zeal, Identity and Meaning: Going to Extremes to Be One Self. In J. Greenberg, S. Koole and T. Pyszczynski (eds.), Handbook of Existential Psychology, pp. 182-199. New York: Guildford

McGregor, I. (2009). Offensive Defensiveness: Toward an Integrative Neuroscience of Compensatory Zeal after Mortality Salience, Personal Uncertainty and Other Poignant Self-Threats. Psychological Inquiry, vol. 17, no. 4, pp. 299-308

McGregor, I.; Prentice, M. and Nash, K. (2009). Personal Uncertainty Management by Reactive Approach Motivation. Psychological Inquiry, vol. 20, no. 4, pp. 225-229

Mill, J. S. (1863). Utilitarianism. Accessed on 12/08/2017 from: https://www.utilitarianism.com/mill1.htm

Morf, C. and Mischel, W. (2012). The Self as a Psycho-Social Dynamic Processing System: Towards a Converging Science of Selfhood. M. Leary and J. Tangney (eds.), Handbook of Self and Identity: Second Edition, pp. 21-49. New York, NY: Guildford

Morris, W. (1999). The Mood System. In D. Kahneman, E. Diener and N. Schwartz (eds.), Wellbeing: The Foundations of Hedonic Psychology, pp. 169-189. New York, NY: Russell Sage Foundation

Munger, M. (2013). Recycling: Can it be Wrong, When It Feels So Right? Cato Unbound, June 2013.

Muriwai, E.; Houkamau, C. and Sibley, C. (2015). Culture as Cure? The Protective Function of Maori Cultural Efficacy on Psychological Distress. New Zealand Journal of Psychology, vol. 44, no. 2, pp. 14-24

Neff, K. and Vonk, R. (2009). Self-Compassion versus Global Self-Esteem: Two Different Ways of Relating to Oneself. Journal of Personality, vol. 77, no. 1, pp. 23-50

Nehamas, A. (1985). Nietzsche: Life as Literature. Cambridge, MA: Harvard University Press.

Nelson, K. (2003). Narrative and Self, Myth and Memory: Emergence of the Cultural Self. In R. Fivush and C. Haden (eds.), Autobiographical Memory and the Construction of a Narrative Self, pp. 3-28. Mahwah, NJ: Erlbaum

Nelson, K. and Lyubomirsky, S. (2012). Finding Happiness: Tailoring Positive Activities for Optimal Well-Being Benefits. In M. Tugade, M. Shiota and L. Kirby (eds.), Handbook of Positive Emotions, pp. 275 - 293. New York, NY: Guildford

Nelson, K. and Lyubomirsky, S. (2016). Gratitude. In S. Friedman, (ed.), Encyclopedia of Mental Health, $2^{\text {nd }}$ Edition, vol. 2, pp. 277-280. Waltham, MA: Academic Press 
Nettle, D.; Harper, Z.; Kidson, A.; Stone, R.; Penton-Voak, I. and Bateson, M. (2013). The Watching Eyes Effect in the Dictator Game: It's Not How Much You Give, It's Being Seen to Give Something. Evolution and Human Behaviour, vol. 34, no. 1, pp. 35-40

Newcomb, M. and Harlow, L. (1986). Life Events and Substance Use among Adolescents: Mediating Effects of Perceived Loss of Control and Meaninglessness in Life. Journal of Personality and Social Psychology, vol. 51, no. 3, pp. 564-577

Ng, W. and Diener, E. (2014). What Matters to the Rich and the Poor? Subjective Well-Being, Financial Satisfaction, and Postmaterialist Needs Across the World. Journal of Personality and Social Psychology, vol. 107 , no. 2 , pp. 326-338

Nietzsche, F. (1881/1996). Daybreak. Trans. R. Hollingdale. Cambridge, UK: Cambridge University Press.

Nietzsche, F. (1891/1978). Thus Spoke Zarathustra: A Book for None and All. New York, NY: Penguin Nietzsche, F. (1885, 1990). The Antichrist. Trans. R. J. Hollingdale. New York, NY: Penguin.

Nietzsche, F. (1886/2000). Beyond Good and Evil: Prelude to a Philosophy of the Future. Trans. W. Kaufmann. New York, NY: Modern Library

Nietzsche, F. (1887/1974). The Gay Science. Second edition. Trans. W. Kaufmann. New York, NY: Vintage Books.

Nietzsche, F. (1888/2000). The Genealogy of Morals: A Polemic. Trans. W. Kaufmann. New York, NY: Modern Library

Nietzsche, F. (1889/1990). The Twilight of the Idols: or How to Philosophise with a Hammer. Trans. R. Hollingdale. New York, NY: Penguin.

Nolen-Hoeksema, S.; Wisco, B. and Lyubomirsky, S. (2008). Rethinking Rumination. Perspectives on Psychological Science, vol. 3, no. 5, pp. 400-424.

Norton, D. (1976). Personal Destinies: A Philosophy of Ethical Individualism. Princeton, NJ: Princeton University Press

Nowlis, V. (1965). Research with Mood Adjective Checklist. In S. Thompkins and C. Isard (eds.), Affect, Cognition and Personality. New York: Springer

Nozick, R. (1974). Anarchy, State and Utopia. New York, NY: Basic Books

Nunnally, J. and Bernstein, I. (1994). Psychometric Theory. New York, NY: McGraw-Hill

Nussbaum, M. (2000). Women and Human Development: The Capabilities Approach. Cambridge, UK: Cambridge University Press

OECD. (2017). Guidelines for Measuring Subjective Well-being. Paris, FR: Organisation for Economic Cooperation and Development.

Okulicz-Kozaryn, A. (2015). Happiness and Place: Why Life is Better Outside the City. Basingstoke, UK: Palgrave Macmillan.

Oswald, A. (2008). On the Curvature of the Reporting Function from Objective Reality to Subjective Feelings. Economics Letters, vol. 100, pp. 369-372

Oswald, A. and Powdthavee, N. (2008). Does Happiness Adapt? A Longitudinal Study of Disability with Implications for Economists and Judges. Journal of Public Economics, vol. 92, no. 5-6, pp. 1061-1077

Oxford University Archives (2007). First Woman Graduate of the University. Bodleian Libraries. Access 18/09/2018 from:

https://www.bodleian.ox.ac.uk/oua/enquiries/first-woman-graduate

Oyserman, D.; Elmore, K. and Smith, G. (2012). Self, Self-Concept and Identity. In M. Leary and J. Tangney (eds.), Handbook of Self and Identity: Second Edition, pp. 21-49. New York, NY: Guildford

Osyerman, D. and Markus, H. (1990). Possible Selves and Delinquency. Journal of Personality and Social Psychology, vol. 59, no. 1, pp. 112-125.

Papageorgeiou, C. and Wells, A. (2004). Depressive Rumination: Nature, Theory and Treatment. Chichester, UK: Wiley

Parfit, D. (1984). Reasons and Persons. Oxford, UK: Clarendon

Pavot, W. and Diener, E. (1993). Review of the Satisfaction with Life Scale. Psychological Assessment, vol. 5, no. 2, pp. 164-172

Phillips, A. and Silvia, P. (2010). Individual Differences in Self-Discrepancies and Emotional Experience: Do Distinct Discrepancies Predict Distinct Emotions? Personality and Individual Differences, vol. 49, no. 2, pp. 148-151

Piaget, J. (1932). The Psychology of Intelligence. Patterson, NJ: Littlefield-Adams

Pinel, E.; Long, A.; Landau, M. and Pyszczynski, T. (2004). I-Sharing, the Problem of Existential Isolation, and Their Implications for Interpersonal and Intergroup Phenomena. In J. Greenberg, S. Koole and T. Pyszczynski (eds.), Handbook of Experimental Existential Psychology, pp. 352-368. New York, NY: Guildford. 
Pinker, S. 2002. The Blank Slate: The Modern Denial of Human Nature. London, UK: Penguin

Polderman, T.; Benyamin, B.; de Leeuw, C.; Sullivan, P.; van Bochoven, A.; Visscher, P.and Posthuma, D. (2015). Meta-Analysis of the Heritability of Human Traits Based on Fifty Years of Twin Studies. Nature Genetics, vol. 47, no. 7, pp. 702-709

Popper, K. (1934/59). The Logic of Scientific Discovery. London, UK: Routledge

Powdthavee, N. (2008). Putting a Price Tag on Friends, Relatives and Neighbours: Using Surveys of Life Satisfaction to Value Social Relationships. The Journal of Socio-Economics, vol. 37, no. 4, pp. 1459-1480

Powdthave, N. (2009). What Happens to People Before and After Disability? Focusing Effects, Lead Effects and Adaptation in Different Areas of Life. Social Science and Medicine, vol. 69, no. 12, pp. 1834-1844

Powdthavee, N. and Stutzer, A. (2014). Economic Approaches to Understanding Change in Happiness. In K. Sheldon and R. Lucas (eds.), Stability of Happiness: Theories and Evidence on Whether Happiness can Change, pp. 219-245. New York: Academic Press

Pyszczynski, T.; Greengerg, J. and Arendt, J. (2012). Freedom versus Fear Revisited: An Integrative Analysis of the Dynamics of the Defense and Growth of Self. In M. Leary and J. Tangney (eds), Handbook of Self and Identity: Second Edition, pp. 378-404. New York, NY: Guildford Press

Reginster, B. (2009). The Affirmation of Life: Nietzsche on Overcoming Nihilism. Cambridge, MA: Harvard University Press

Reis, H.; Sheldon, K.; Gable, S.; Roscoe, J. and Ryan, R. (2000). Daily Well-Being: The Role of Autonomy, Competence and Relatedness. Personality and Social Psychology Bulletin, vol. 26, no. 4, pp. 419-435

Reker, G. and Peacock, E. (1981). The Life Attitude Profile (LAP): A Multidimensional Instrument for Assessing Attitudes towards Life. Canadian Journal of Behavioural Science, vol. 13, no. 3, pp. 264-273

Ricard, M. (2003). Happiness: A Guide to Developing Life's Most Important Skill. London, UK: Atlantic Books. Ricard, M. (2014). A Buddhist View of Happiness. Journal of Law and Religion, vol. 29, no. 1, pp. 14-29

Robbins, L. (1934). An Essay on the Nature and Significance of Economic Science. London: MacMillan and Co.

Rochat, P. (2003). Five Levels of Self-awareness as they unfold in Early Life. Consciousness and cognition: an international journal, vol. 12, no. 4, pp. 717-731

Roemer, L.; Lee, J.; Salters-Pedneault, K.; Erisman, S.; Orsillo, S. and Mennin, D. (2009). Mindfulness and Emotion Regulation Difficulties in Generalized Anxiety Disorder: Preliminary Evidence for Independent and Overlapping Contributions. Behavioural Theory, vol. 40, no. 2, pp. 142-154.

Roth, G.; Shahar, B.; Zohar-Shefer, Y.; Benita, M.; Moed, A.; Bibi, U.; Kanat-Maymon, Y.; and Ryan, R. (2017). Benefits of Emotional Integration and Costs of Emotional Distancing. Journal of Personality, advance online publication, doi: 10.1111/jopy.12366

Routledge, C. and Arendt, J. (2009). Creative Terror Management: Creativity as a Facilitator of Cultural Exploration after Mortality Salience. Personality and Social Psychology Bulletin, vol. 35, no. 4, pp. 493-505

Røysamb, E.; Bang Nes, R. and Vitters $\varnothing$, J. (2014). Well-Being: Heritable and Changeable. In K. Sheldon and R. Lucas (eds.), Stability of Happiness: Theories and Evidence on Whether Happiness can Change, pp. 9-36. New York: Academic Press

Ruberton, P. M.; Kruse, E. and Lyubomirsky, S. (2016). Boosting State Humility via Gratitude, Self-Affirmation and Awe: Theoretical and Empirical Perspectives. In E. Worthington, D. Davis, and J. Hook (eds.), Handbook of Humility. London, UK: Routledge

Ruttenberg, T. (2013). Wellbeing Economics and Buen Vivir: Development Alternatives for Inclusive Human Security. The Fletcher Journal of Human Security, vol. 33, no. 1, pp. 68-93

Ruiz-Mirazo, K.; Etxeberria, A.; Moreno, A. and Ibàñez, J. (2000). Organisms and Their Place in Biology. Theory in Biosciences, vol. 119, no. 3-4, pp. 209-233

Russell, B. (1930). The Conquest of Happiness. London, UK: Routledge

Ryan, R. (1993). Agency and Organization: Intrinsic Motivation, Autonomy and the Self in Psychological Development. In J. Jacobs (ed.), Nebraska Symposium on Motivation, vol. 40, pp. 1-56. Lincoln, NE: University of Nebraska Press.

Ryan, R. and Deci, E. (2000). Self-determination Theory and the Facilitation of Intrinsic Motivation, Social Development and Well-being. American Psychologist, vol. 55, pp. 68-78

Ryan, R. and Deci, E. (2004). Autonomy Is No Illusion: Self-Determination Theory and the Empirical Study of Authenticity, Awareness and Will. In J. Greenberg, S. Koole and T. Pyszczynski (eds.), Handbook of Experimental Existential Psychology, pp. 431-448. New York, NY: Guildford

Ryan, R. \& Huta, V. (2009). Happiness as Healthy Functioning or Happiness as Wellbeing: The Importance of Eudaimonic Thinking (Response to the Kashdan et al. and Waterman discussion). The Journal of Positive Psychology, vol. 4, no. 3, pp. 202-204 
Ryan, R. and Deci, E. (2017). Self-Determination Theory: Basic Psychological Needs in Motivation, Development, and Wellness. New York, NY: Guildford.

Ryan, R., Deci, E. and Huta, V. (2008). Living Well: A Self-Determination Theory Perspective on Eudaimonia. Journal of Happiness Studies, vol. 9, no. 1, pp. 139-170

Ryan, R.; Deci, E. and Vansteenkiste, M. (2016). Autonomy and Autonomy Disturbances in Self-Development and Psychopathology: Research on Motivation, Attachment, and Clinical Process. In D. Cichetti (ed.), Developmental Psychopathology, vol. 1, pp. 385-438. Hoboken, NJ: John Wiley and Sons Inc.

Ryff, C. (1989a). Beyond Ponce de Leon and Life Satisfaction: New Directions in Quest of Successful Aging. International Journal of Behavioural Development, vol. 12, no. 1, pp. 35-55

Ryff, C. (1989b). Happiness Is Everything, or Is It? Explorations on the Meaning of Psychological Well-Being. Journal of Personality and Social Psychology, vol. 57, no. 6, pp. 1069-1081

Ryff, C. and Singer, B. (2008). Know Thyself and Become What You Are: A Eudaimonic Approach to Psychological Well-Being. Journal of Happiness Studies, vol. 9, no. 1, pp. 13-39

Ryff, C. (2012). Existential Well-Being and Health. In P. Wong (ed.), The Human Quest for Meaning: Theories, Research and Applications, $2^{\text {nd }}$ edition, pp. 233-248. London, UK: Routledge

Salzman, M. and Halloran, M. (2004). Cultural Trauma and Recovery: Cultural Meaning, Self-Esteem, and the Reconstruction of the Cultural Anxiety Buffer. In J. Greenberg, S. Koole and T. Pyszczynski (eds.), Handbook of Experimental Existential Psychology, pp. 231-246. New York, NY: The Guildford Press

Samuelson, P. (1938). A Note on the Pure Theory of Consumers' Behaviour. Economica, vol. 5, no. 17, pp. 61-71.

Samuelson, P. (1948). Consumption Theory in Terms of Revealed Preference. Economica: New Series, vol. 15, no. 60 , pp. 243-245

Santelices, B. (1999). How Many Kinds of Individuals Are There? Trends in Ecology and Evolution, vol. 14, no. 4, pp. $918-931$

Sartre, J. (1938/2000). Nausea. Trans. R. Baldick. New York, NY: Penguin.

Sartre, J. (1943/2005). Being and Nothingness. Trans. H. Barnes. London, UK: Routledge

Sartre, J. and De Beauvoir, S. (1946). Existentialism is a Humanism. Trans. P. Mairet. Retrieved from: https://www.marxists.org/reference/archive/sartre/works/exist/sartre.htm

Schacter, S. and Singer, J. (1962). Cognitive, Social and Physiological Determinants of Emotional State. Psychological Review, vol. 69, no. 5, pp. 379-399

Schartz, B.; Ward, A.; Lyubomirsky, S.; Monterosso, J.; White, K. and Lehman, D. (2002). Maximizing versus Satisficing: Happiness is a Matter of Choice. Journal of Personality and Social Psychology, vol. 83, no. 5, pp. 1178-1197

Schkade, D. and Kahneman, D. (1998). Does Living in California Make People Happy? A Focussing Illusion in Judgements of Life Satisfaction. Psychological Science, vol. 9, no. 5, pp. 340-346.

Schopenhauer, A. (1844/1966). The World as Will and Representation, vol. 2. New York, NY: Dover Publications

Schreiber, C. and Kahneman, D. (2000). Determinants of the Remembered Utility of Aversive Sounds. Journal of Experimental Psychology, vol. 129, no. 1, pp. 27-42

Schwarz, C.; Bode, R.; Repucci, N.; Becker, J.; Sprangers, M. and Fayers, P. (2006). The Clinical Significance of Adaptation to Changing Health: A Meta-Analysis of Response Shift. Quality of Life Research, vol. 15, no. 9, pp. 1533-1550

Schwarz, N. and Clore, G. (1983). Mood, Misattribution, and Judgements of Well-Being: Informative and Directive Functions of Affective States. Journal of Personality and Social Psychology, vol. 45, no. 3, pp. 513-523

Schwarz, N.; Strack, F. and Mai, H. (1991). Assimilation and Contrast Effects in Part-Whole Question Sequences: A Conversational Logic Analysis. Public Opinion Quarterly, vol. 55, no. 1, pp. 3-23

Sedikides, C.; Gaertner, L. and Vevea, J. (2005). Pancultural Self-Enhancement Reloaded: A Meta-Analytic Reply to Heine (2005). Journal of Personality and Social Psychology, vol. 89, no. 4, pp. 539-551.

Seligman, M. (1992). Helplessness: On Depression, Development and Death. New York, NY: Freeman

Seligman, M.; Ernest, R.; Gillham, J.; Reivich, K. and Linkins, M. (2009). Positive Education: Positive Psychology and Classroom Interventions. Oxford Review of Education, vol. 35, no. 3, pp. 293-311

Seligman, M. (2012). Flourish: A Visionary New Understanding of Happiness and Well-Being. Melbourne, AU: William Heineman Australia.

Selman, R. (2003). The Promotion of Social Awareness. New York, NY: Russell Sage Sen, A. (1999a). Development as Freedom. Oxford, UK: Oxford University Press Sen, A. (1999b). Commodities and Capabilities. Oxford, UK: Oxford University Press Shapiro, D. (1981). Autonomy and Rigid Character. New York, NY: Basic Books 
Shapiro, S.; Carlson, L.; Astin, J. and Freedman, B. (2006). Mechanisms of Mindfulness. Journal of Clinical Psychology, vol. 62, no. 3, pp. 373-386

Sharpe, P.; Martin, N. and Roth, K. (2011). Optimism and the Big Five Factors of Personality: Beyond Neuroticism and Extroversion. Personality and Individual Differences, vol. 51, no. 8, pp. 946-951

Shaver, P. and Mikulincer, M. (eds.) (2012). Meaning, Mortality and Choice: The Social Psychology of Existential Concerns. Washington, DC: American Psychological Association.

Sheldon, K. (2002). The Self-Concordance Model of Healthy Goal Striving: When Personal Goals Correctly Represent the Person. In E. Deci and R. Ryan, Handbook of Self-Determination Research, pp. 65-86, Rochester, NY: Rochester University Press.

Sheldon, K. (2013). Individual Daimon, Universal Needs, and Subjective Well-Being: Happiness as the Natural Consequence of a Life Well-Lived. In A. Waterman (ed.), The Best Within Us: Positive Psychological Perspectives on Eudaimonia, Washington, DC: American Psychological Association.

Sheldon, K. and Kasser, T. (1995). Coherence and Congruence: Two Aspects of Personality Integration. Journal of Personality and Social Psychology, vol. 68, no. 3, pp. 531-543

Sheldon, K. and Elliot, A. (1998). Not All Personal Goals are Personal: Comparing Autonomous and Controlled Reasons for Goals and Predictors of Effort and Attainment. Personality and Social Psychology Bulletin, vol. 24, no. 5, pp. 546-557

Sheldon, K. and Houser-Marko, L. (2001). Self-Concordance, Goal Attainment, and the Pursuit of Happiness: Can There Be an Upward Spiral? Journal of Personality and Social Psychology, vol. 80, no. 1, pp. 152-165

Sheldon, K. and Vansteenkiste, M. (2005). Personal Goals and Time Travel: How are Future Places Visited, and Is It Worth It? In A. Strathman and J. Joireman (eds.), Understanding Behaviour in the Context of Time: Theory, Research and Application, pp. 143-163. Mahwah, NJ: Erlbaum.

Sheldon, K. and Cooper, M. (2008). Goal Striving Within Agentic and Communal Roles: Functionally Independent Pathways to Enhanced Well-Being. Journal of Personality, vol. 76, no. 3, pp. 415-448

Sheldon, K. and Schuler, J. (2011). Needing, Wanting, and Having: Integrating Motive Disposition Theory and Self-Determination Theory. Journal of Personality and Social Psychology, vol. 101, no. 5, pp. 1106-1123

Sheldon, K and Krieger, L. (2014). Service Job Lawyers are Happier than Money Job Lawyers, Despite Their Lower Income. Journal of Positive Psychology, vol. 90, no. 3, pp. 219-226.

Sheldon, K. M. and Lucas. R. E. (2014). Stability of Happiness: Theories and evidence on whether happiness can change, London: Academic Press.

Sheldon, K. and Lyubomirsky, S. (2012).The Challenge of Staying Happier: Testing the Hedonic Adaptation Prevention Model. Personality and Social Psychology Bulletin, vol. 38, no. 5, pp. 670-680

Sheldon, K.; Ryan, R. and Reis, H. (1996). What Makes for a Good Day? Competence and Autonomy in the Day and in the Person. Personality and Social Psychology Bulletin, vol. 22, no. 12, pp. 1270-1279.

Sheldon, K.; Elliot, A.; Ryan, R.; Chirkov, V.; Kim, Y.; Wu, C.; Demir, M. and Sun, Z. (2004). Self-Concordance and Subjective Well-Being in Four Cultures. Journal of Cross Cultural Psychology, vol. 35, no. 2, pp. 209-223

Sheldon, K.; Ryan, R.; Deci, E. and Kasser, T. (2004). The Independent Effects of Goal Contents and Motives on Well-Being: It's Both What You Pursue and Why You Pursue It. Personality and Social Psychology Bulletin, vol. 30, no. 4, pp. 475-486

Sheldon, K.; Abad, N.; Ferguson, Y; Gunz, A.; Houser-Marko, L. Nichols, C.; Lyubomirsky, S. (2009). Persistent Pursuit of Need-Satisfying Goals Leads to Increased Happiness: A 6-month Experimental Longitudinal Study. Motivation and Emotions, vol. 34, no. 1, pp. 39-48

Sheldon, K. M., Boehm, J. and Lyubomirsky, S. (2013). Variety is the Spice of Life: The Hedonic Adaptation Prevention Model. In I. Boniwell, S. David and A. Conley Ayers (eds.), The Oxford Handbook of Wellbeing, pp. 901-914. Oxford, UK: Oxford University Press.

Shin L. and Lyubomirsky, S. (2014). Positive Activity Interventions for Mental Health Conditions: Basic Research and Clinical Applications. In J. Johnson and A. Wood (eds.), The Handbook of Positive Clinical Psychology, pp. 349-363. New York, NY: Wiley

Shower, C. (1992a). Compartmentalization of Positive and Negative Self-Knowledge: Keeping Bad Apples out of the Bunch. Journal of Personality and Social Psychology, vol. 62, no. 6, pp. 1036-1049

Showers, C. (1992b). Evaluatively Integrative Thinking about Characteristics of the Self. Personality and Social Psychology Bulletin, vol. 18, no. 6, pp. 719-729

Showers, C. (2002). Integration and Compartmentalization: A Model of Self-Structure and Self-Change. In D. Cervone and W. Mishcel (eds.), Advances in Personality Science, pp. 271-291. New York: Guildford 
Showers, C. and Zeigler-Hill, V. (2012). Organisation of Self-Knowledge: Features, Functions and Flexibility. In M. Leary and J. Tangney (eds.), Handbook of Self and Identity: Second Edition, pp. 105-123. New York, NY: Guildford

Sides, J.; Tesler, M. and Vavreck, L. (2016). Identity Crisis: The 2016 Presidential Campaign and the Battle for the Meaning of America. Princeton, NJ: Princeton University Press

Silvia, P. and Eddington, K. (2012). Self and Emotion. In M. Leary and J. Tangney (eds.), Handbook of Self and Identity: Second Edition, pp. 425 - 445. New York, NY: Guildford

Simmel, G. (1903/1950). The Metropolis and Mental Life. Trans. K. Wolff. New York, NY: Free Press.

Simon, M. (2017). Want a Robot to Walk Like You Do? Don't Expect it to Look Human. Wired, retrieved on 08/11/2018 from: https://www.wired.com/story/want-a-robot-to-walk-like-you-dont-expect-it-to-look-human/

Simon, L.; Arendt, J.; Greenberg, J.; Pyszczynski, T. amd Solomon, S. (1998). Terror-Management and Meaning: Evidence that the Opportunity to Defend the Worldview in Response to Mortality Salience Increases the Meaningfulness of Life in the Mildly Depressed. Journal of Personality, vol. 66, no. 3, pp. 359-382

Sin, N. and Lyubomirsky, S. (2009). Enhancing Well-Being and Alleviating Depressive Symptoms with Positive Psychology Interventions: A Practice-Friendly Meta-Analysis. Journal of Clinical Psychology, vol. 65, no. 5, pp. $467-487$

Singer, J. (2004). Narrative Identity and Meaning Making across the Adult Lifespan: An Introduction. Journal of Personality, vol. 72, no. 3, pp. 437-460

Slattery, J. and Park, C. (2012). Clinical Approaches to Discrepancies in Meaning: Conceptualization, Assessment and Treatment. In P. Wong (ed.), The Human Quest for Meaning: Theories, Research and Applications, $2^{\text {nd }}$ edition, pp. 497 - 520. London, UK: Routledge

Slavin, M. and Kreigman, D. (1992). The Adaptive Design of the Human Psyche: Psychoanalysis, Evolutionary Biology, and the Therapeutic Process. New York, NY: Guildford Smith, A. (1759). The Theory of Moral Sentiments. Edinburgh: Kincaid.

Smith, D.; Schwarz, N.; Robert, T. and Ubel, P. (2006). Why Are You Calling Me? How Study Introductions Change Response Patterns. Quality of Life Research, vol. 15, no. 4, pp. 621-630.

Smyth, J.; True, N. and Souto, J. (2001). Effects of Writing about Traumatic Experiences: The Necessity for Narrative Structuring. Journal of Social and Clinical Psychology, vol. 20, no. 2, pp. 161-172

Soloman, S. (2012). The Social Psychology of Meaning, Mortality and Choice: An Integrative Perspective on Existential Concerns. In P. Shaver and M. Mikulincer (eds.), Meaning, Mortality and Choice: The Social Psychology of Existential Concerns, pp. 401-418. Washington, DC: American Psychological Association.

Sorrentino, R. and Roney, C. (2000). The Uncertain Mind: Individual Differences in Facing the Unknown. Philadelphia, PA: Psychology Press.

Sorrentino, R.; Yang, Y. and Szeto, A. (2009). Uncertainty Management: To Fear or Not to Fear? Psychological Inquiry, vol. 20, no. 4, pp. 240-244

Specht, J.; Egloff, B. and Schmukle, S. (2011). The Benefits of Believing in Chance or Fate: External Locus of Control as a Protective Factor for Coping with the Death of a Spouse. Social Psychological and Personality Science, vol. 2, no. 2, pp. 132-137

Springer, K. and Hauser, R. (2006). An Assessment of the Construct Validity of Ryff's Scale of Psychological Well-Being: Method, Mode, and Measurement Effects. Social Science Research, vol. 35, no. 4, pg. 1080-1102

Springer, K.; Hauser, R. and Freese, J. (2006). Bad News Indeed for Ryff's Six-Factor Model of Well-Being. Social Science Research, vol. 35, no. 4, pp. 1120-1131

Stephenson, B. and Wolfers, J. (2013). Subjective Well-Being and Income: Is There Any Evidence of Satiation? American Economic Review, vol. 103, no. 3, pp. 598-604

Steel, P.; Schmidt, J. and Shultz, J. (2008). Refining the Relationship between Personality and Subjective WellBeing. Psychology Bulletin, vol. 134, no. 1, pp. 138-61.

Steele, C.; Spencer, S.; and Lynch, M. (1993). Self-Image Resilience and Dissonance: The Role of Affirmational Resources. Journal of Personality and Social Psychology, vol. 64, no. 6, pp. 885-896.

Steger, M. (2006). An Illustration of Issues in Factor Extraction and Identification of Dimensionality in Psychological Assessment Data. Journal of Personality Assessment, vol. 86, no. 3, pp. 263-272

Steger, M. (2010). Experiencing Meaning in Life: Optimal Functioning at the Nexus of Well-Being, Psychopathology, and Spirituality. In V. Wong (ed.), The Human Quest for Meaning: Theories, Research and Applications, pp. 165-184. London, UK: Routledge.

Steger, M. and Frazier, P. (2005). Meaning in Life: One Link in the Chain from Religion to Well-Being. Journal of Counselling Psychology, vol. 52, no. 4, pp. 574-582 
Steger, M.; Frazier, P.; Oishi, S. and Kaler, M. (2006). The Meaning in Life Questionnaire: Assessing the Presence of and Search for Meaning in Life. Journal of Counseling Psychology, vol. 53, no. 1, pp. 80-93

Steger, M.; Kashdan, T. and Oishi, S. (2008). Being Good by Doing Good: Eudaimonic Activity and Daily WellBeing Correlates, Mediators and Temporal Relations. Journal of Research in Personality, vol. 42, no. 1, pp. 2242

Steger, M.; Kashdan, T.; Sullivan, B. and Lorentz, D. (2008). Understanding the Search for Meaning in Life: Personality, Cognitive Style, and the Dynamic between Seeking and Experiencing Meaning. Journal of Personality, vol. 76, no. 2, pp. 199-228

Stevens, S. (1946). On the Theory of Scales of Measurement. Science, vol. 103, pp. 677-680

Stevenson, B. and Wolfers, J. (2009). The Paradox of Declining Female Happiness. American Economic Journal: Economic Policy, vol. 1, no. 2, pp. 190-225

Stevenson, B. and Wolfers, J. (2013). Subjective Well-Being and Income: Is There Any Evidence of Satiation? American Economic Review, vol. 103, no. 3, pp. 598-604

Stiglitz, J., Sen, A. and Fitoussi, J. (2009). Report by the Commission on the Measurement of Economic Performance and Social Progress. Paris, FR: Commission on the Measurement of Economic Performance and Social Progress.

Stillman, S.; Gibson, J.; McKenzie, D. and Rohorua, H. (2015). Miserable Migrants? Natural Experiment Evidence on International Migration and Objective and Subjective Well-Being. World Development vol. 65, issue C pp. 79-93

Stone, A. and Mackie, C. (eds.) (2013). Subjective Well-Being: Measuring Wellbeing, Suffering and Other Dimensions of Experience. National Research Council Report. Washington DC: The National Academies Press.

Stone, A.; Shiffman, S. and DeVries, M. (1999). Ecological Momentary Assessment. In D. Kahneman, E. Diener and N. Schwarz (eds.), Well-Being: The Foundations of Hedonic Psychology, pp. 26-39. New York, NY: Russell Sage

Strack, L.; Martin, N. and Schwarz, N. (1988). Priming and Communication: Social Determinants of Information Use in Judgements of Life Satisfaction. European Journal of Social Psychology, vol. 18, no. 5, pp. 429-442

Strauss, C.; Lever Taylor, B.; Gu, J.; Kuyken, W.; Baer, R.; Jones, F. and Cavanagh, K. (2016). What is Compassion and How Can We Measure It? A Review of Definitions and Measures. Clinical Psychology Review, vol. 47, no. 1, pp. 15-27

Stronge, S.; Sengupta, N.; Barlow, F.; Osborne, D.; Houkamau, C. and Sibley, C. (2016). Perceived Discrimination Predicts Increased Support for Political Rights and Life Satisfaction Mediated by Ethnic Identity: A Longitudinal Analysis. Cultural Diversity and Ethnic Minority Psychology, vol. 22, no. 3, pp. 359-368

Stutzer, A. and Frey, B. (2006). Does Marriage Make People Happy, Or Do Happy People Get Married? Journal of Socio-Economics, vol. 35, no. 2, pp. 326-347

Summerfield, P. (2012). Women Workers in the Second World War: Production and Patriarchy in Conflict. London, UK: Routledge.

Sumner, L. (1999). Welfare, Happiness and Ethics. Oxford, UK: Oxford University Press

Sumner, R.; Burrow, A. and Hill, P. (2014). Identity and Purpose as Predictors of Subjective Well-Being in Emerging Adulthood. Emerging Adulthood, vol. 3, no. 1, pp. 46-54

Swann, W. (2011). Self-Verification Theory. In P. Van Lange, A. Kruglanski and E. Tory-Higgins (eds.), Handbook of Theories of Social Psychology, vol. 2, pp. 23-42. Thousand Oaks, CA: Sage

Swann, W. and Buhrmester, M. (2012). Self-Verification: The Search for Coherence. In M. Leary and J. Tangney (eds.), Handbook of Self and Identity: Second Edition, pp. 405-424. New York, NY: Guildford

Tangney, J. and Tracy, J. (2012). Self-Conscious Emotions. In M. Leary and J. Tangney (eds.), Handbook of Self and Identity: Second Edition, pp. 446-478

Tangney, J.; Niedenthal, P.; Covert, M. and Barlow, D. (1998). Are Shame and Guilt Related to Distinct SelfDiscrepancies? A Test of Higgins' 1987 Hypothesis. Journal of Personality and Social Psychology, vol. 75, no. 1, pp. 256-268

Tesser, A. (2000). On the Confluence of Self-Esteem Maintenance Mechanisms. Personality and Social Psychology Review, vol. 4, no. 4, pp. 290-299

Thirlwall, A. and Pacheco-López, P. (2017). Economics of Development: Theory and Evidence, $10^{\text {th }}$ Edition. London UK: MacMillan International

Thompson, R. (2006). The development of the Person: Social Understanding, Relationships, Conscience, Self. In N. Eisenberg, W. Damon and R. Lerner (eds.), Handbook of Child Psychology-Volume 3: Social, Emotional and Personality Development, 6th edition, pp. 24-98. New York, NY: Wiley

Tiberius, V. (2008). The Reflective Life: Living Wisely Within our Limits. Oxford, UK: Oxford University Press 
Tomasello, M.; Carpenter, M.; Call, J.; Behne, T. and Moll, H. (2005). Understanding and Shared Intentions: Origins of Cultural Cognition. Behavioural and Brain Sciences, vol. 28, no. 5, pp. 675-691

To, S. and Sung, W. (2017). Presence of Meaning, Sources of Meaning, and Subjective Well-Being in Emerging Adulthood: A Sample of Hong Kong Community College Students. Emerging Adulthood, vol. 5, no. 1, pp. 6974

Trapnell, P. and Campbell, J. (1999). Private Self-Consciousness and the Five-Factor Model of Personality: Distinguishing Rumination from Reflection. Journal of Personality and Social Psychology, vol. 76, no. 2, pp. 284-304

Tryon, W. and Radzin, A. (1972). Purpose in Life as a Function of Ego-Resiliency, Dogmatism and Biographical Variables. Journal of Clinical Psychology, vol. 28, no. 4, pp. 544-545

Tugade, M. and Fredrickson, B. (2004). Resilient Individuals Use Positive Emotions to Bounce Back from Negative Emotional Experiences. Journal of Personality and Social Psychology, vol. 86, no. 2, pp. 320-333

Tversky, A. and Kahneman, D. (1986). Rational Choice and the Framing of Decisions. The Journal of Business, vol. 59, no. 4, Part 2, pp. S251-S278

Ubel, P.; Jankovic, A.; Smith, D.; Langa, K. and Fagerlin, A. (2005). What is Perfect Health to an 85 Year-Old? Evidence for Scale Norming in Subjective Health Ratings. Medical Care, vol. 43, no. 10, pp. 1054-1057

Ubel, P.; Peeters, Y. and Smith, D. (2010). Abandoning the Language of "Response Shift": A Plea for Conceptual Clarity in Distinguishing Scale Norming from True Changes in Quality of Life. Quality of Life Research, vol. 19 , no. 4, pp. 465-471

United Nations (2015a). The Millennium Development Goals Report 2015. New York, NY: United Nations.

United Nations (2015b). Transforming Our World: The 2030 Agenda for Sustainable Development. New York, NY: United Nations

Van Boven, L. and Gilovich, T. (2003). To Do or To Have? That is the Question. Journal of Social and Personality Psychology, vol. 85, no. 6, pp. 1193-1202

Van den Bos, K. (2009). Making Sense of Life: The Existential Self Trying to Deal with Personal Uncertainty. Psychological Inquiry, vol. 20, no. 4, pp. 197-217

Van den Bos, K. (2009). On the Psychology of the Uncertain Self and the Integration of the Worldview Defence Zoo. Psychological Inquiry, vol. 20, no. 4, pp. 252-261

Van Dierendonck, D. (2004). The Construct Validity of Ryff's Scales of Psychological Well-Being and its Extension with Spiritual Well-Being. Personality and Individual Differences, vol. 36, no. 3, pp. 629-643

Van Praag, B. and Frijters, P. (1999). The Measurement of Welfare and Wellbeing: The Leyden Approach. In E. Diener, D. Kahneman and N. Schwartz (eds.), Wellbeing: The Foundations of Hedonic Psychology, pp. 413433. New York, NY: Russell Sage Foundation

Van Praag, B. and Ferrer-i-Carbonell, A. (2004). Happiness Quantified: A Social Calculus Approach. Oxford, UK: Oxford University Press

Vansteenkiste, M.; Duriez, B.; Simons, J. and Soenens, B. (2006). Materialistic Values and Well-Being Among Business Students: Further Evidence of their Detrimental Effects. Journal of Applied Social Psychology, vol. 36, no. 12, pp. 2892-2908

Veblen, T. (1899). The Theory of the Leisure Class. Retrieved from: http://moglen.law.columbia.edu/LCS/theoryleisureclass.pdf

Vess, M.; Arndt, J. and Schlegel, R. (2011). Abstract Construal Levels Attenuate State Self-Esteem Reactivity. Journal of Experimental Social Psychology, vol. 47, no. 4, pp. 861-864

Vittersø, J.; Oelmann, H. and Wang, A. (2009). Life Satisfaction is Not a Balanced Estimator of the Good Life. Evidence from Reaction Time Measures and Self-Reported Emotions. Journal of Happiness Studies, vol. 10, no. 1, pp. $1-17$

Vitters $\emptyset$, J.; Søholt, Y; Hetland, A.; Thorsen, I and Røysamb, E. (2010). Was Hercules Happy? Some Answers from a Functional Model of Human Well-Being. Social Indicators Research, vol. 95, no. 1, pp. 1-18

Vitters $\emptyset$, J. (2013). Feelings, Meanings, and Optimal Functioning: Some Distinctions Between Hedonic and Eudaimonic Well-Being. In A. Waterman (ed.), The Best Within Us: Positive Psychology Perspectives on Eudaimonia, pp. 39-56. Washington, DC: American Psychological Association.

Vittersø, J. (2014). Functional Well-Being: Happiness as Feelings, Evaluations and Functioning. In I. Boniwell and S. David (eds.), The Oxford Handbook of Happiness, pp. 227-244. Oxford, UK: Oxford University Press. Vitrano, C. (2014). The Nature and Value of Happiness. Boulder, CO: Westview Press

Waldfogel, J.; Craigie, T. and Brooks-Gunn, J. (2010). Fragile Families and Child Well-Being. Future Child, vol. 20, no. 2, pp. 87-112 
Wallace, H. and Tice, D. (2012). Reflect Appraisal through a $21^{\text {st }}$-Century Looking Glass. In M. Leary and J. Tangney (eds.), Handbook of Self and Identity: Second Edition, pp. 124-140. New York, NY: Guildford.

Waterman, A. (1990). Personal Expressiveness: Philosophical and Psychological Foundations. Journal of Mind and Behaviour, vol. 11, no. 1, pp. 47-74

Waterman, A. (1992). Identity as an Aspect of Optimal Psychological Functioning. In T. Gullota and R. Montemajor (eds.), Identity Formation during Adolescence: Advances in Adolescent Development, vol. 4, pp. $50-72$

Waterman, A. (1993). Two Conceptions of Happiness: Contrasts of Personal Expressiveness (Eudaimonia) and Hedonic Enjoyment. Journal of Personality and Social Psychology, vol. 64, no. 4, pp. 678-691

Waterman, A. (2007a). Doing Well: The Relationship of Identity Status to Three Conceptions of Well-Being. Identity: An International Journal of Theory and Research, vol. 7, no. 4, pp. 289-307

Waterman, A. (2007b). On the Importance of Distinguishing Hedonia and Eudaimonia when Considering the Hedonic Treadmill. American Psychologist, vol. 62, no. 6, pp. 612-613

Waterman, A. (2008). Reconsidering Happiness: A Eudaimonist's Persective. The Journal of Positive Psychology, vol. 3, no. 4, pp. 234-252

Waterman, A. (ed). (2013). The Best Within Us: Positive Psychology Perspectives on Eudaimonia. Washington, DC: American Psychological Association

Weijers, D. (2014). Nozick's Experience Machine is Dead, Long Live the Experience Machine! Philosophical Psychology, vol. 27, no. 4, pp. 513-535

Weimann, J, Knabe, A and Schöb, R. (2015). Measuring Happiness: The Economics of Well-Being. Cambridge, MA: MIT Press

Weinstein, N. and Ryan, R. (2011). A Self-Determination Theory Approach to Understanding Stress Incursion and Responses. Stress and Health, vol. 27, no. 1, pp. 4-17

Weinstein, N.; Ryan, R. and Deci, E. (2012). Motivation, Meaning, and Wellness: A Self-Determination Perspective on the Creation and Internationalization of Personal Meanings and Life Goals. In P. Wong (ed.), The Human Quest for Meaning: Theories, Research and Applications, $2^{\text {nd }}$ edition, pp. 81-106. London, UK: Routledge

Whillans, A.; Dunn, E.; Smeets, P.; Bekkers, R. and Norton, M. (2017). Buying Time Promotes Happiness. PNAS Early View

White, M. and Dolan, P. (2009). Accounting for the Richness of Daily Activities. Psychological Science, vol. 20, no. 8, pp. 1000-1008

Wilson, D. (2002). Darwin's Cathedral: Evolution, Religion, and the Nature of Society. Chicago, IL: University of Chicago Press

Wilson, D. (2015). Does Altruism Exist? Culture, Genes, and the Welfare of Others. New Haven, CT: Yale University Press.

Winkelmann, L. and Winkelmann, R. (1998). Why are the Unemployed So Unhappy: Evidence from Panel Data. Economica, vol. 65, no. 257, pp. 1-15

Witter, R.; Stock, W.; Okun, M. and Haring, M. (1985). Religion and Subjective Well-Being: A Quantitative Synthesis. Review of Religious Studies, vol. 26, no. 4, pp. 332-342.

Wood, A.; Froh, J. and Geraghty, W. (2010). Gratitude and Well-Being: A Review and Theoretical Integration. Clinical Psychology Review, vol. 30, no. 7, pp. 890-905

Woodard, C. (2012). Classifying Theories of Welfare. Philosophical studies, vol. 165, no. 3, pp. 787-803.

Woodard, C. (2015). Hybrid Theories. In G. Fletcher (ed.), Routledge Handbook of Philosophy of Wellbeing, pp. 161-175. London, UK: Routledge

Woodman, T. and Hemmings, S. (2008). Body Image Self-Discrepancies and Affect: Exploring the Feared Body Self. Self and Identity, vol. 7, no. 4, pp. 413-429

Wong, V. (ed.), (2010). The Human Quest for Meaning: Theories, Research and Applications. London, UK: Routledge.

Yap, M. and Yu, E. (2016). Operationalising the Capability Approach: Developing Culturally Relevant Indicators of Indigenous Well-Being-An Australian Example. Oxford Development Studies, vol. 44, no. 3, pp. 315-331

Yap, S., Anusic, I. and Lucas, R. (2014). Does Happiness Change? Evidence from Longitudinal Studies. In K. Sheldon and R. Lucas (eds.), Stability of Happiness: Theories and Evidence on Whether Happiness can Change, pp. 129-146. New York, NY: Academic Press.

Yalçin, I. and Malkoç, A. (2015). The Relationship between Meaning in Life and Subjective Well-Being: Forgiveness and Hope as Mediators. Journal of Happiness Studies, vol. 16, no. 4, pp. 915-929 


\section{APPENDIX}

Figure A1: Total change as depicted on plots

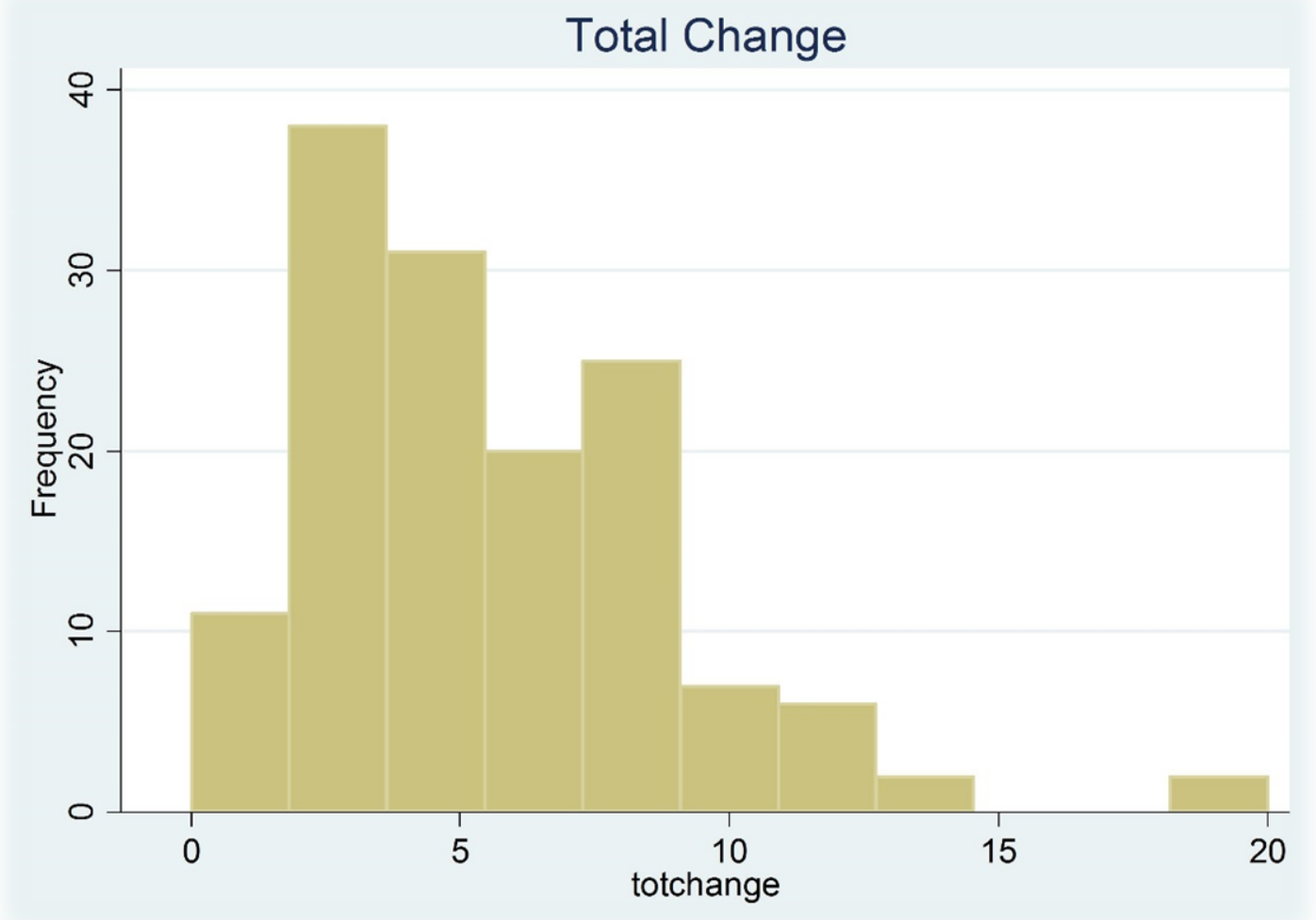

Figure A2: Total change over time in scale responses, HILDA

\section{Total Change - HILDA}

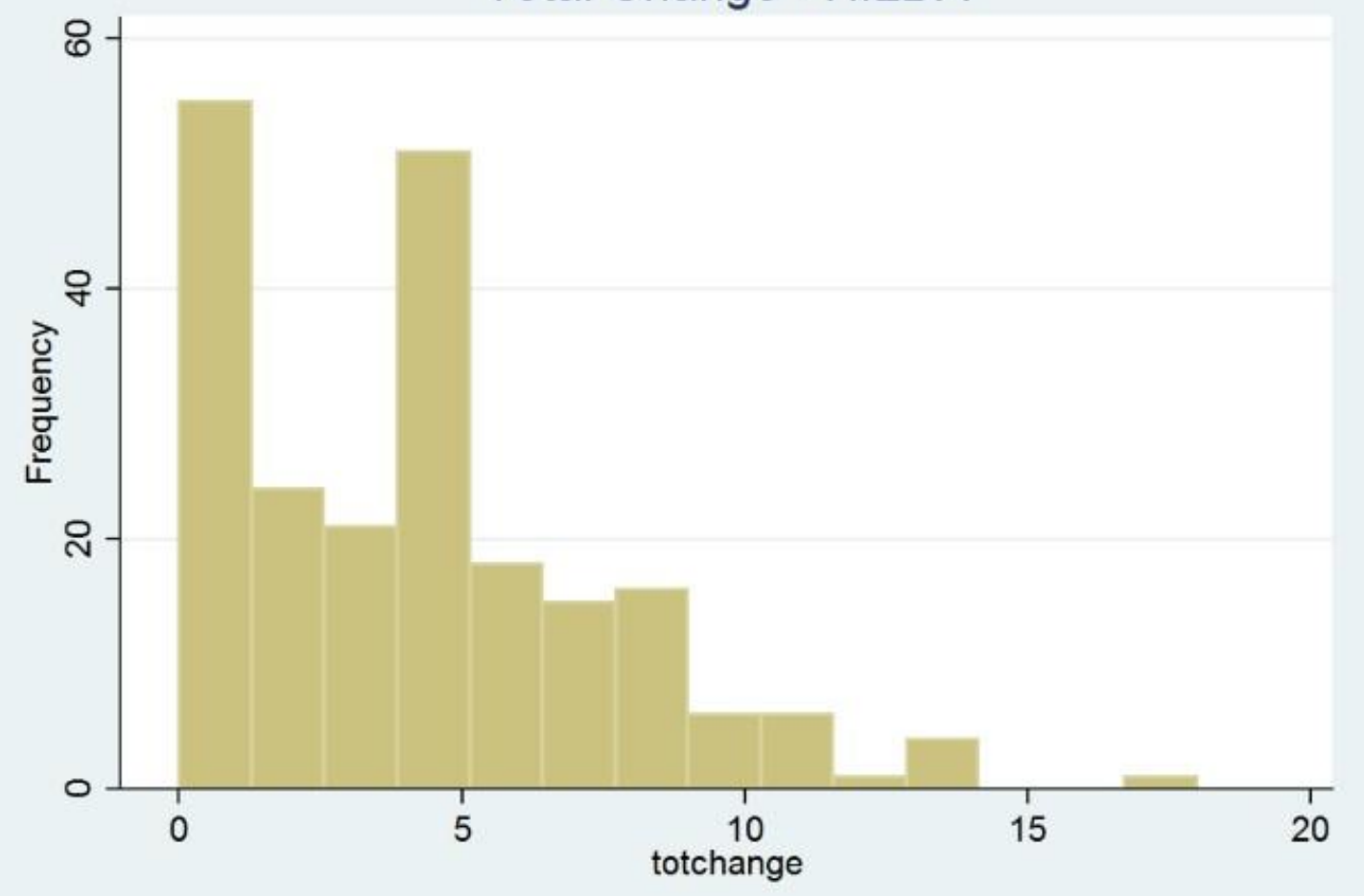


Figure A3: Netchange as depicted on plots

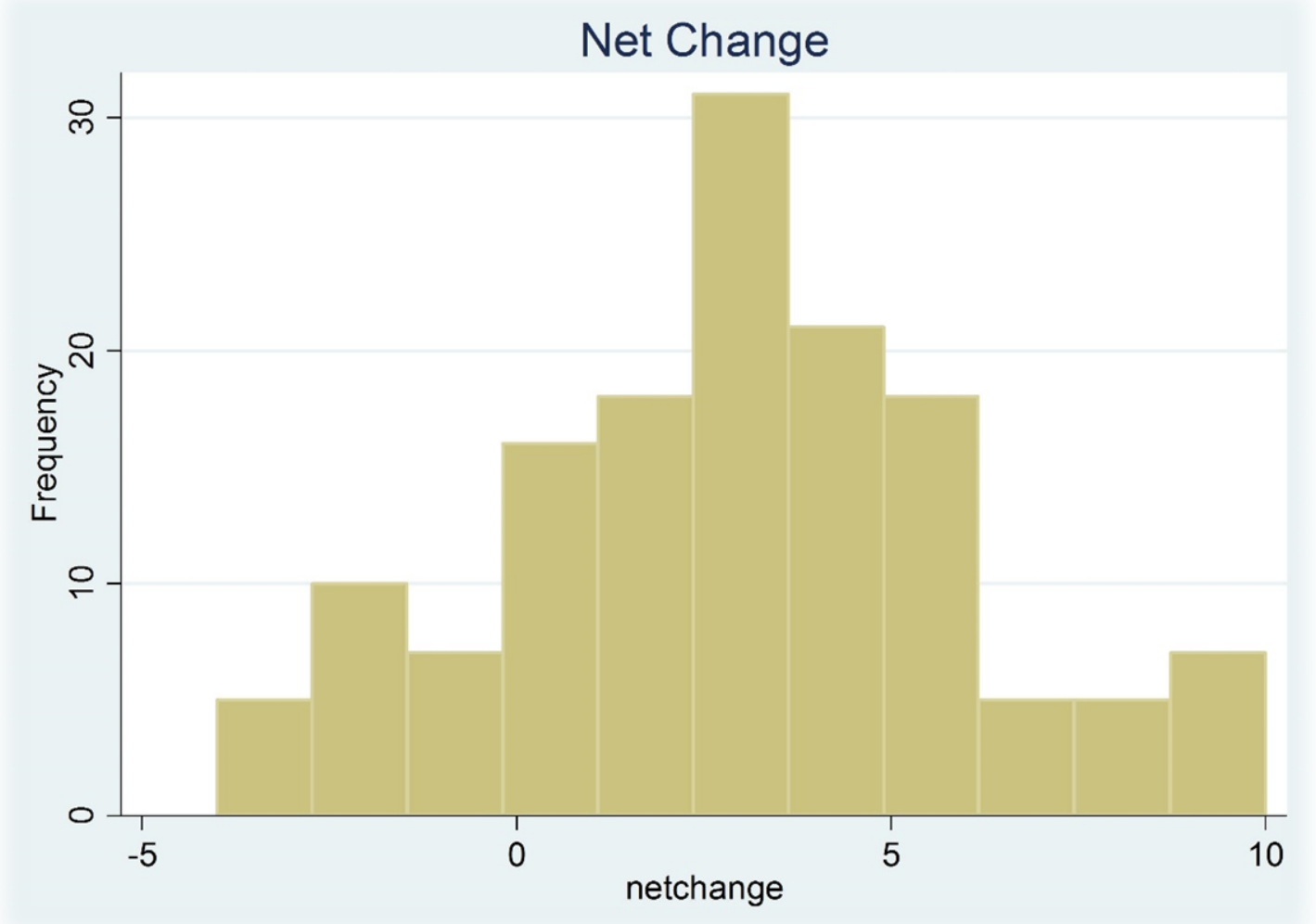

Figure A4: Scale response change in plotting study

Change in Scale

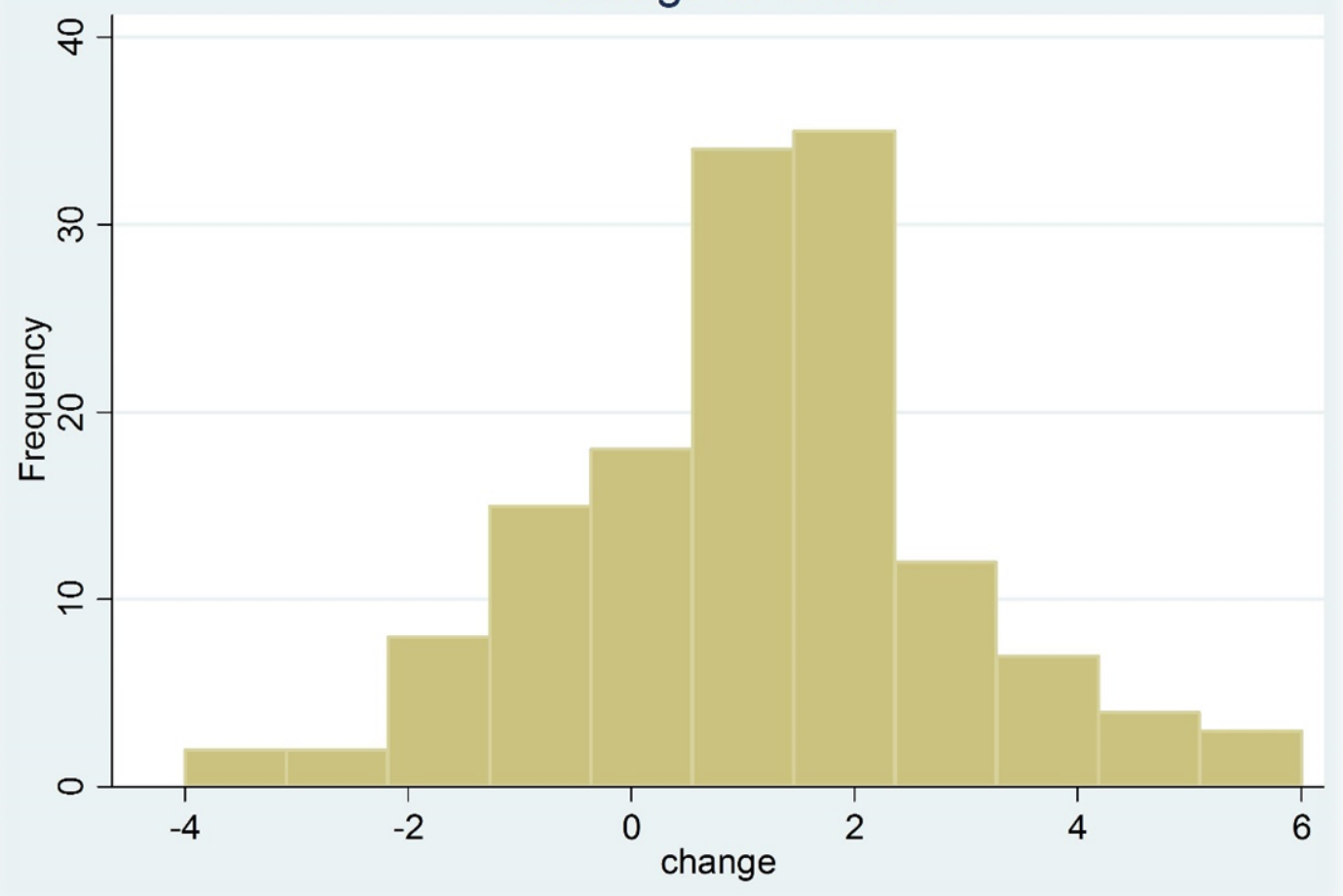


Figure A5: Scale response change between wave 10 and wave 1 in HILDA sample

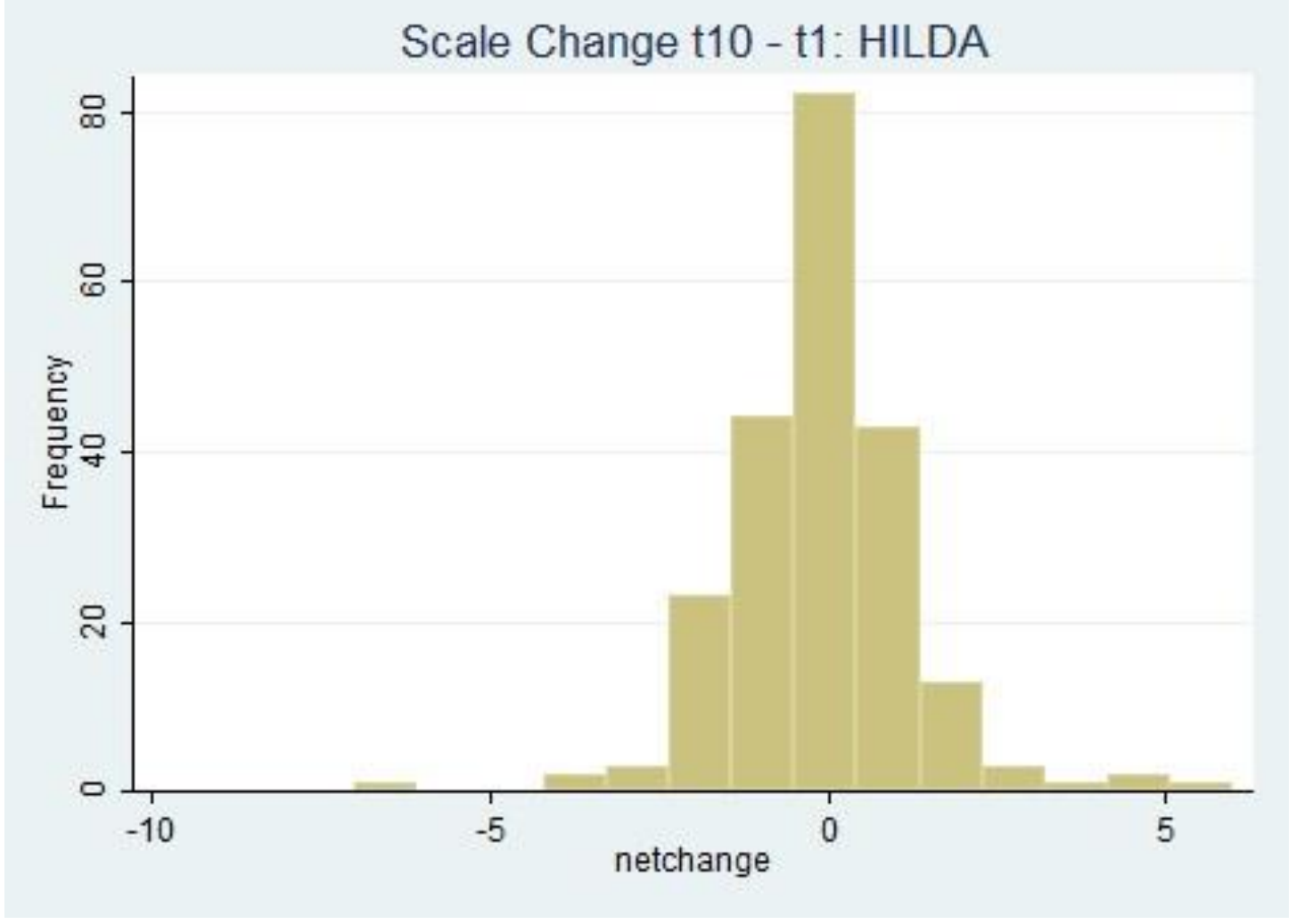

Figure A6: Consistency of plotting responses and scale responses

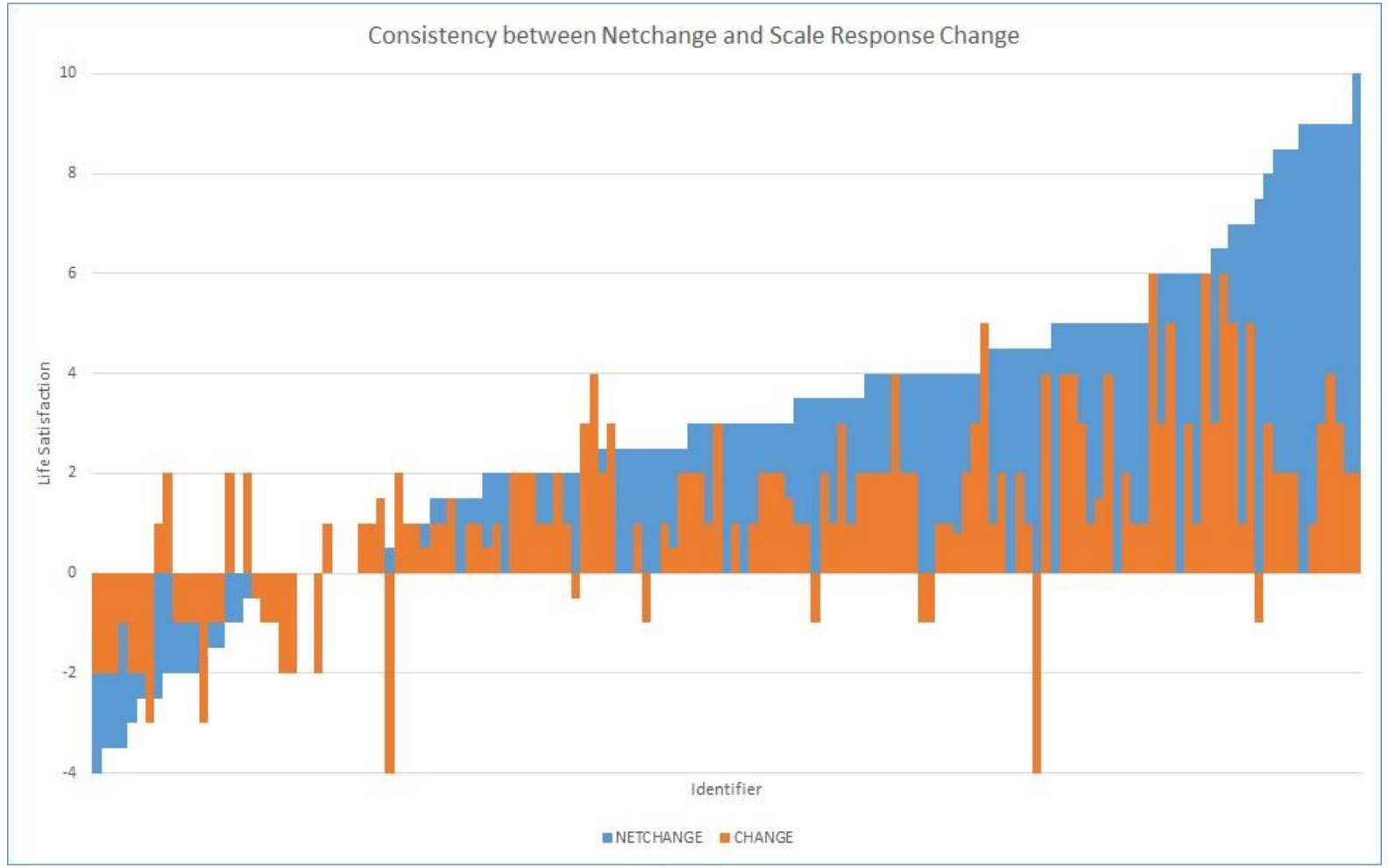

